

\section{DISTRIBUTION SHEET}

To

Distribution

Project TitleMrork Order

CSER 00-006: Storage of Plutonium Residue Containers in 55-Gallon Drums at the PFP

From

Criticality and Shielding FFS Name

Fluor Hanford

J. D. Carlson

D. A. Conners IV

G. R. Franz

E. J. Lipke

B. S. Mo

L. Dayley

B. D. Skeels

DOE-RL

S. J. Altschuler

I. T. Nirider

Docket Files (2 copies)

Central Files (Orig. +2 copies)

DOE/RL Reading Room

\section{Fluor Federal Services}

K. D. Dobbin

D. G. Erickson

J. P. Estrellado Jr.

S. R. Gedeon

H. J. Goldberg

I. E. Johnson

E. M. M1ller

A. B. Rau (3)

R. F. Richard

K. N. Schwinkendorf

H. Toffer

A. D. Wilkinson

D. W. Footan $\begin{array}{llll}\text { Page } 1 & \text { of } 1\end{array}$

Date $5 / 8 / 00$

EDT No. 619959

ECN No. N/A

\begin{tabular}{|c|c|c|c|c|}
\hline MSIN & $\begin{array}{l}\text { Text } \\
\text { Whit All } \\
\text { Attach. }\end{array}$ & Text Only & $\begin{array}{l}\text { Attach.I } \\
\text { Appendix } \\
\text { Only }\end{array}$ & $\begin{array}{c}\text { EDT/ECN } \\
\text { Only }\end{array}$ \\
\hline T5-57 & $\mathrm{x}$ & & & \\
\hline T4-15 & $x$ & & & \\
\hline$A 0-26$ & $x$ & & & \\
\hline$A 0-26$ & $x$ & & & \\
\hline T5-54 & $x$ & & & \\
\hline$T 4-20$ & $x$ & & & \\
\hline T5-09 & $\mathbf{x}$ & & & \\
\hline & & & & \\
\hline R3-77 & $\mathrm{x}$ & & & \\
\hline A5-55 & $x$ & & & \\
\hline A3-02 & $\mathrm{x}$ & & & \\
\hline B1-07 & $\mathbf{x}$ & & & \\
\hline $\mathrm{H} 2-53$ & $x$ & & & \\
\hline & & & & \\
\hline & & & & \\
\hline B4-44 & $\mathrm{x}$ & & & \\
\hline B4-44 & $x$ & & & \\
\hline B4-44 & $x$ & & & \\
\hline B4-44 & $x$ & & & \\
\hline B4-44 & $x$ & & & \\
\hline B4-45 & $x$ & & & \\
\hline$B 4-44$ & $x$ & & & \\
\hline B4-46 & $x$ & & & \\
\hline B4-44 & $x$ & & & \\
\hline B4-44 & $x$ & & & \\
\hline B4-44 & $x$ & & & \\
\hline B4-44 & $x$ & & & \\
\hline B4-44 & $x$ & & & \\
\hline & & & & \\
\hline
\end{tabular}




\section{INFORMATION CLEARANCE FORM}

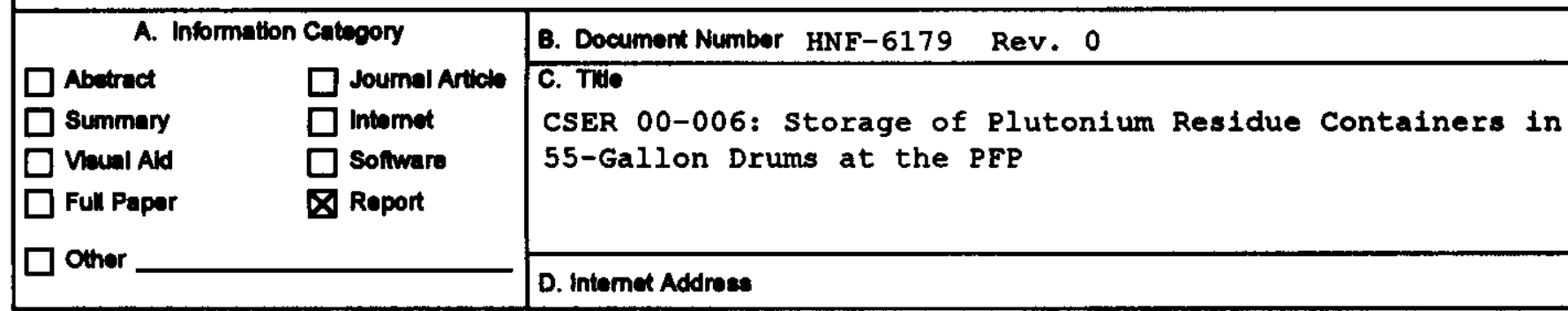

E. Required Information

1. Is document potentlally Claceilied? ONo OYes (MANDATORY Teren Telpe s72/00 Managers dsinture Required

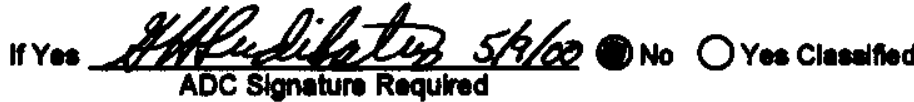

2. Internal Review Required? if Yes, Document Signatures Below ONo OYes

Couneal

Program

3. References in the Information are Applied Technology ONo OYes Export Controlled Information
4. Does Information Contuin the Following: (MANDATORY)
a. Now or Novel (Patontable) subject Mattor?
Ono Ores

If "Yos". Obecloeure No.:

b. Information Recelved in Confidence, such as Propribtary and/or Inventions?

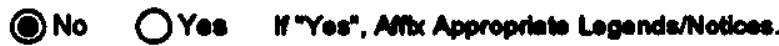

c. Copyrights? ONo OYes II"Yos", Aluch Pormbation.

d. Trademarks? ONo OYes I"Yes", kdentily in Document.

5. is Information requiring submiscion to OSTI? $O$ No $O$ Yos If Yes UC- 2000 and $B \& R-\underline{E N 7002010}$

6. Release Lever? $O$ Public $O$ Limited

7. Charge Code 110844

F. Complete for a Journal Article

1. Tite of Joumal $N / A$

G. Complete for a Presentation

1. Twe for Conference or Meeting $N / A$

2. Group Sponeoring $\mathrm{N} / \mathrm{A}$

3. Date of Conference $N / A$

4. City/State $\mathrm{N} / \mathrm{A}$

5. Wil Information be Publiahed in Procasedinge? $O$ No OYes

6. Wil Material be Handed Out? $O$ No $O$ Yes

H. Author/Requetor

K. D. Dobbin KX Dofhin 5-9-00

(Print and Sign) Responeible Manager

H. Toffer (Print and Sign)
I. Roviowere
Yes Print
Signature
Genoral Couneal
Orinos of External Afratre
DOE-RL
othor LMSI \& bAREN NOLAND
Other
$\square$
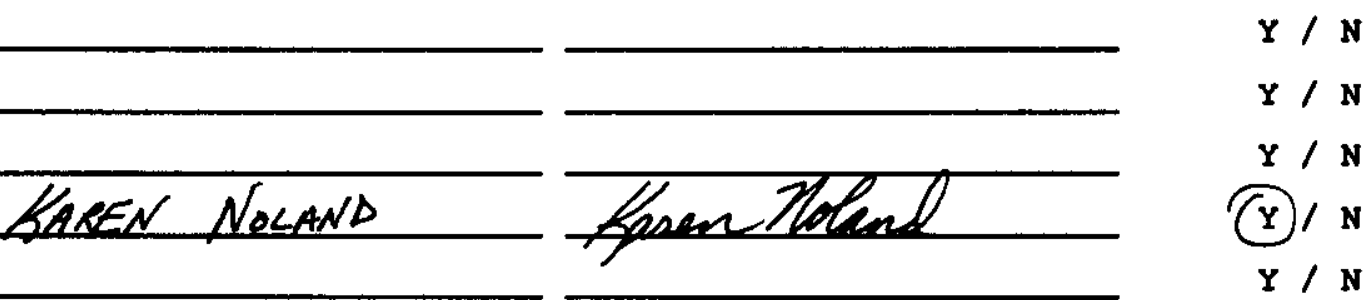

Public YN (II N, complete J)

J. If Information Includes Senclive information and is not to be released to the Public indicate category below.
$\square$ Applied Technology
$\square$ Protected CRADA
$\square$ Perconalpivate
$\square$ Export Controlied
$\square$ Propriotary
$\square$ Procurement-Senultive
$\square$ Businese-seneltive
$\square$ Patentable
$\square$ Predecisional
$\square$ Other (Specily)
$\square$ UCNI

K. If Additional Comments, Please Attach Separate Sheet

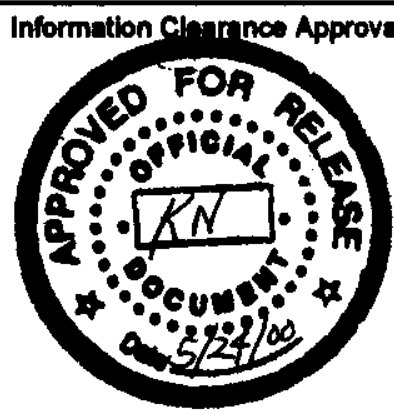




\section{CSER 00-006: Storage of Plutonium Residue. Containers in 55-Gallon Drums at the PFP}

Prepared for the U.S. Department of Energy Assistant Secretary for Environmental Management

Project Hanford Management Contractor for the

U.S. Department of Energy under Contract DE-AC06-96RL13200

Fluor Hanford

P.O. Box 1000

Richland, Weshington 


\section{CSER 00-006: Storage of Plutonium Residue Containers in 55-Gallon Drums at the PFP}

K. D. Dobbin

H. J. Goldberg

Fluor Federal Services

Date Published

May 2000

Prepared for the U.S. Department of Energy

Assistant Secretary for Environmental Management

Project Hanford Management Contractor for the

U.S. Department of Energy under Contract DE-AC06-96RL13200

Fuar Hanford

P.O. Box 1000

Richland, Washington
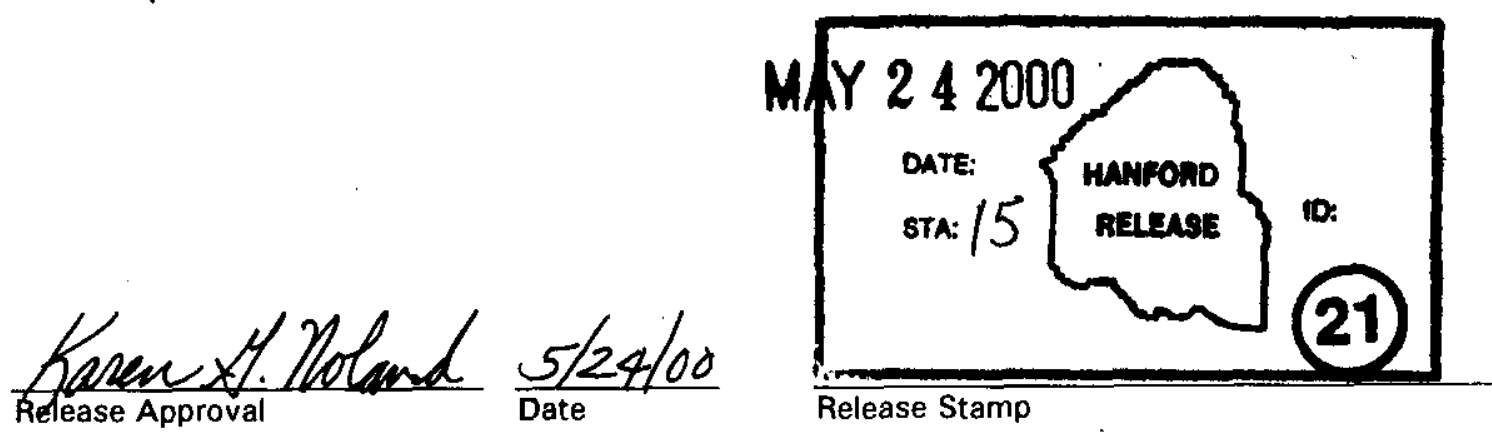
TRADEMARK DISCLAIMER

Reference herein to any specific commercial product, process, or service by trade name, trademark, manufacturer, or

otherwise, does not necessarily constitute or imply its

endorsement, recommendation, or favoring by the United

States Government or any agency thereof or its contractors or subcontractors.

This report has been reproduced from the best available copy.

Printed in the Unlted States of America

Total Pages: $\frac{142}{N N}$ 


\section{CSER 00-006: Storage of Plutonium Residue Containers in 55-Gallon Drums at the PFP}

Key Words: 234-5Z, PFP, wagon, 55-Gallon, residue, billet drums, concrete walls

Abstract: This criticality safety evaluation report (CSER) provides the required limit set and controls for safe transit and storage of these drums in the 234-5Z Building at the PFP. A mass limit of $200 \mathrm{~g}$ of plutonium or fissile equivalent per drum is acceptable provided that the drums are not stacked and that the general transportation and storage requirements at the PFP are met. However, a wagon is likely to pass by a drum during transit so one wagon is placed next to a drum as one of the base cases. PFP transition and storage spacing requirements are still required to provide consistent facility-wide requirements but a spacing violation between a wagon and a drum does not constitute a violation of double contingency. Waste drums that contain a maximum of $200 \mathrm{~g}$ per drum mass limit can be intermixed in the billet drum array. Drums are excluded from Rooms 227, Room 230C, and the vaults. Also, FFTF fuel can be stored in the room 192A, adjacent to billet drums stored in Room 192D, due to the thickness of the concrete walls isolating the drums from the fuel. 


\title{
CSER 00-006: STORAGE OF PLUTONIUM RESIDUE CONTAINERS IN 55 GALLON DRUMS AT THE PFP
}

\author{
May 2000 \\ Prepared by \\ Fluor Federal Services, Inc. \\ Richland, Washington \\ For \\ Fluor Hanford, Inc. \\ In Support of \\ Task Order No. 5204
}

Prepared by: $12 \&$ Sobbin

Date: $5-8-00$

K. D. Dobbin, Criticality and Shielding

Prepared by:

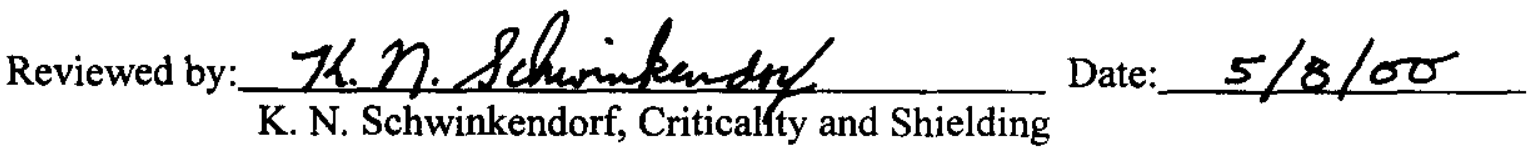

Approved by:

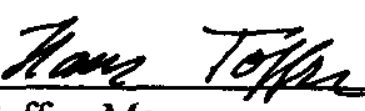

Date: $578 / 00$

H. Toffer, Manager

Criticality and Shielding 
HNF-6179 Rev. 0

This page intentionally left blank. 


\section{Executive Summary}

The plutonium stabilization program at the Plutonium Finishing Plant (PFP) involves treatment of the residual inventory of plutonium at the plant by various means, dependent on the material form and makeup, to convert it into forms amenable to long term storage at PFP or for disposition elsewhere. There is a significant quantity of plutonium-bearing residues stored in various vaults and gloveboxes. The residues are in the forms of sand, slag and crucible (SS\&C), ash, or oxide and other miscellaneous residues from prior process operations. These materials, along with other fissile residues are to be stabilized by the cementation process as cement billets or direct packaged and stored in 55-gallon drums. The storage of this residual plutonium in these drums at the PFP is addressed by this criticality safety evaluation report (CSER).

This criticality safety evaluation report (CSER) provides the required limit set and controls for safe transit and storage of these drums in the 234-5Z Building at the PFP. A mass limit of $200 \mathrm{~g}$ of plutonium or fissile equivalent per drum is acceptable provided that the drums are not stacked and that the general transportation and storage requirements at the PFP are met. However, a wagon is likely to pass by a drum during transit so one wagon is placed next to a drum as one of the base cases. PFP transition and storage spacing requirements are still required to provide consistent facility-wide requirements but a spacing violation between a wagon and a drum does not constitute a violation of double contingency. Waste drums that contain a maximum of $200 \mathrm{~g}$ per drum mass limit can be intermixed in the billet drum array. Drums are excluded from Room 227, Room 230C, and the vaults. Also, FFTF fuel can be stored in the room 192A, adjacent to billet drums stored in Room 192D, due to the thickness of the concrete walls isolating the drums from the fuel.

Evaluation of these drums included normal, base cases, and contingencies. The base cases included an array of drums and a single drum passing a wagon in the hallway. Each contingency is evaluated assuming the unlikely event happens to the most conservative base case. A hazards assessment was conducted to assure that each credible unlikely event or set of correlated unlikely events was included in this analysis. This evaluation shows that storage of cemented billets as described in this report meets the double contingency requirement. That is, at least two unlikely, independent, and concurrent changes in process conditions are required before a criticality is possible. Therefore, this CSER meets the requirements for a criticality evaluation contained in the Hanford Site Nuclear Criticality Safety Manuals, HNF-PRO-334 (FDH 2000), HNF-PRO-537 (FDH 1997a), and HNF-PRO-539 (FDH 1997b), ANSI standards (ANSI 1998), and DOE Order 5480.24 (DOE 1992). This CSER also follows Fluor Federal Services, Inc. Practice 134.290.1121 (FFS 1999). 
HNF-6179 Rev. 0

This page intentionally left blank. 
TABLE OF CONTENTS

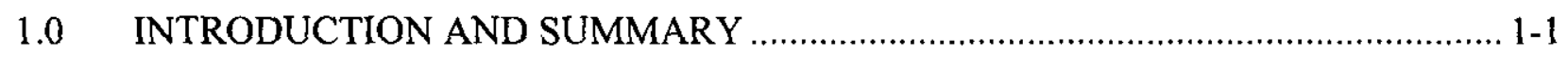

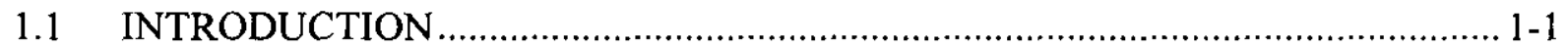

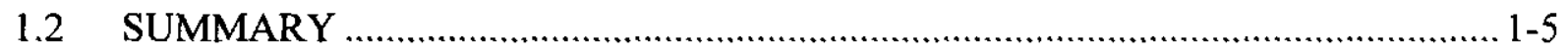

2.0 SYSTEM DESCRIPTION AND NORMAL OPERATIONS ................................... 2-1

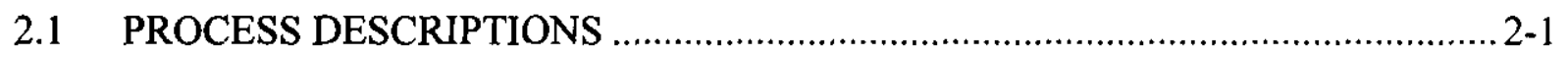

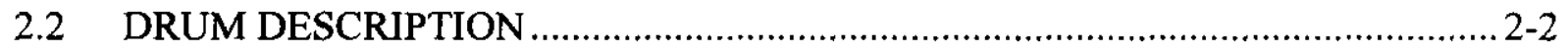

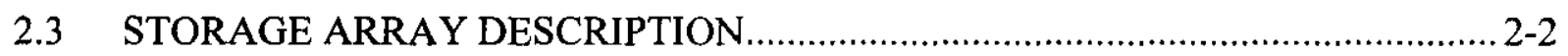

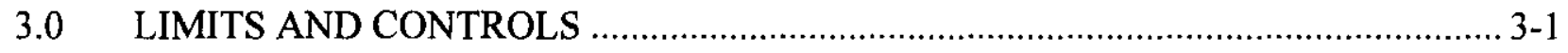

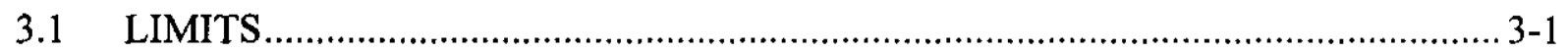

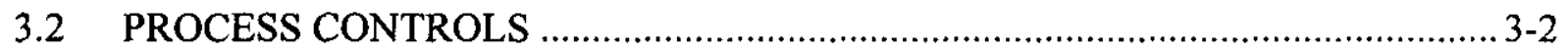

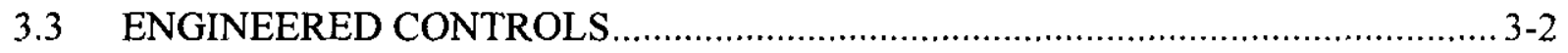

3.4 ADMINISTRATIVE CONTROL IMPLEMENTATION ……........................... 3-2

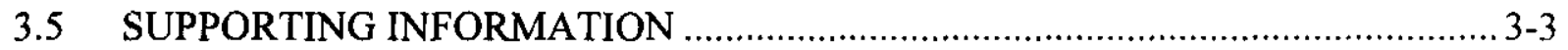

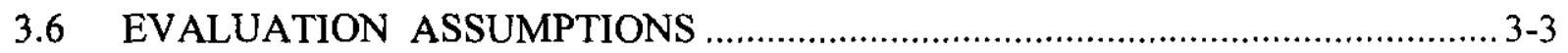

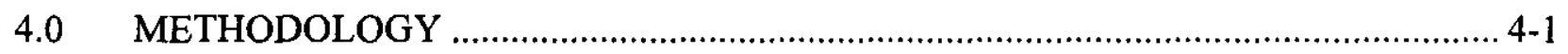

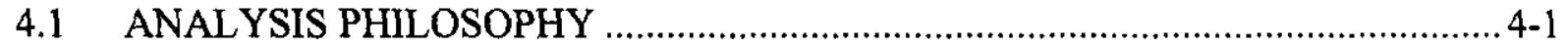

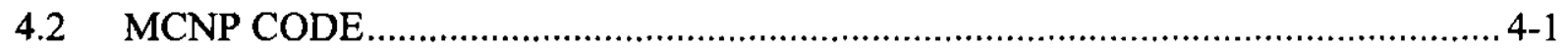

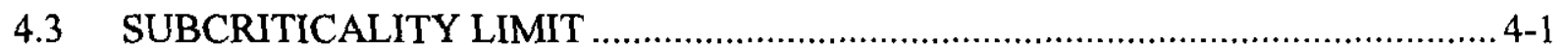

4.4 APPLICATION OF DOUBLE CONTINGENCY PRINCIPLE ………................. 4-2

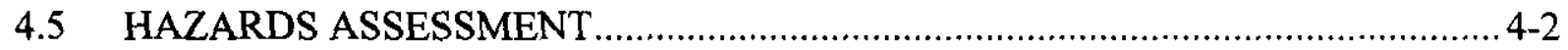

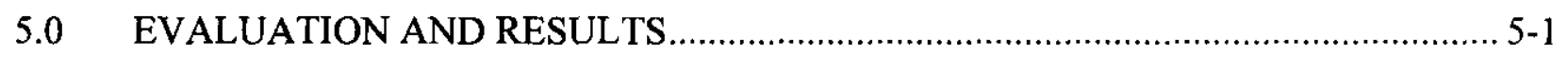

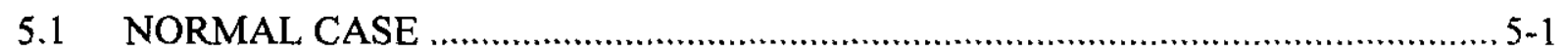

5.2 BASE CASE

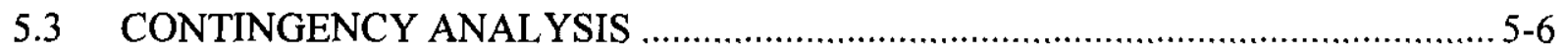

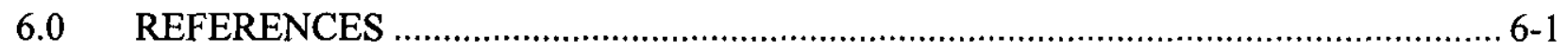

APPENDIX A - INDEPENDENT REVIEW COMMENTS AND CHECKLIST ……............ A-1

APPENDIX B - MCNP COMPUTER CODE VALIDATION ……....................................

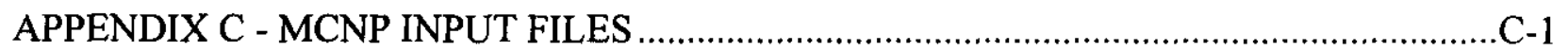

APPENDIX D - PRELIMINARY HAZARDS ANALYSIS …………..............................

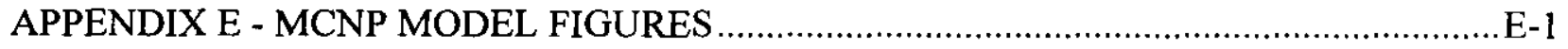




\section{List of Tables}

Table 1-1. Plutonium Residue in 55 Gallon Drums Base Case Summary...................................1-3

Table 1-2. Plutonium Residue in 55 Gallon Drum Contingency Summary ...............................1-4

Table 3-1. Controls on Parameters Related to Criticality Safety for Plutonium Billets in 55 Gallon Drums.......................................................................................... $3-1$

Table 3-2. Limit Set for Storage of Plutonium Residue in 55 Gallon Drums .............................3-1

Table 5-1. Elemental Weight Fractions and Densities of Materials used in Calculations...............5-2

Table 5-2. Base Case Model for Infinite Array ...............................................................5-4

Table 5-3. MCNP Calculational Results of Base Case of Infinite Array of Drums .......................5-4

Table 5-4. Base Case Model for Drum Next to Wagon .........................................................5-5

Table 5-5. MCNP Calculational Results of Base Case of a Drum Next to a Wagon ......................5-5

Table 5-6. Contingency Case Model for Two Layer Infinite Array .........................................5-8

Table 5-7. Calculational Results for Two Layer Infinite Array ............................................5-8

Table 5-8. Contingency Case Model for Overbatching ......................................................5-9

Table 5-9. Calculational Results for Overbatching .........................................................5-9

Table 5-10. Contingency Case Model for Wagon Next to Drum Array .......................................5-10

Table 5-11. Calculational Results for Wagon Next to Drum Array ............................................5-10

\section{List of Figures}

Figure 1: $\mathrm{k}_{\mathrm{eff}}$ for $200 \mathrm{~g}$ in Three Billets in a 55 Gallon Drum Versus Density of Polyethylene Fill. 
HNF-6179 Rev. 0

\section{List of Terms}

$\begin{array}{ll}\text { AIChE } & \text { American Institute of Chemical Engineers } \\ \text { ANSI } & \text { American National Standards Institute } \\ \text { ANS } & \text { American Nuclear Society } \\ \text { CSER } & \text { Criticality Safety Evaluation Report } \\ \text { DOE } & \text { United States Department of Energy } \\ \text { ID } & \text { Identification Number } \\ \text { MCM } & \text { Minimum Critical Mass } \\ \text { NC } & \text { No Controls } \\ \text { NDA } & \text { Nondestructive Analysis } \\ \text { PFP } & \text { Plutonium Finishing Plant } \\ \text { PHA } & \text { Preliminary Hazards Analysis } \\ \text { PnG } & \text { Pipe-and-Go } \\ \text { POC } & \text { Pipe Overpack Container } \\ \text { SS\&C } & \text { Sand, Slag, and Crucible }\end{array}$


HNF-6179 Rev. 0

This page intentionally left blank.

viii 


\subsection{INTRODUCTION AND SUMMARY}

\subsection{INTRODUCTION}

The plutonium stabilization program at the Plutonium Finishing Plant (PFP) involves treatment of the residual inventory of plutonium at the plant by various means, dependent on the material form and makeup, to convert it into forms amenable to long term storage at PFP or for disposition elsewhere. There is a significant quantity of plutonium-bearing residues stored in various vaults and gloveboxes. The residues are in the forms of sand, slag and crucible (SS\&C), ash, or oxide and other miscellaneous residues from prior process operations. These materials, along with other fissile residues are to be stabilized by the cementation process as cement billets or direct packaged and stored in 55-gallon drums. The storage of this residual plutonium in these drums at the PFP is addressed by this criticality safety evaluation report (CSER).

SS\&C material will be stabilized for storage and ultimate disposal by cementation with portland cement to form concrete billets in Glovebox HA-20MB in Room 235B. The product of that operation is placed in $2.7 \mathrm{~L}$ containers containing up to $200 \mathrm{~g}$ plutonium or fissile equivalent. The volume of these containers is defined in Section 2.1. They are either bagged out of the HA-20MB glovebox or out of the adjacent HA-28 conveyor glovebox, then placed into 55-gallon drums.

The other residues will either be cemented in Glovebox HA-20MB or directly packaged by the Pipe-and-Go (PnG) process. Irregardless of form, the residue will be loaded into 55-gallon drums and stored in Building 234-5Z. The plutonium or fissile equivalent will be limited to $200 \mathrm{~g}$ per drum and drums are excluded from Room 227 , Room $230 \mathrm{C}$, and the vaults.

The criticality safety evaluation includes the loading, transport, and storage of these drums in Building 234-5Z. Mass will be controlled to $200 \mathrm{~g}$ plutonium or fissile equivalent per drum. Drums will not be stacked, due to the prohibition on stacking throughout the PFP complex. These billet drums can be intermingled with other waste drums that also do not exceed the $200 \mathrm{~g}$ plutonium mass limit. The general PFP transition and storage spacing requirements will apply between these drums and other fissile material to provide consistent facility-wide requirements. However, a transport wagon is included in the base calculation so that if a wagon should violate that spacing requirement to a single drum, double contingency would not be compromised. If uranium is present in this scrap, its enrichment must not exceed $50 \mathrm{wt} \%{ }^{235} \mathrm{U}$. Each of these limitations has contingent events analyzed with the exception of the ${ }^{235} \mathrm{U}$ enrichment because it has been determined during the hazards evaluation of CSER 00-001 (Dobbin 2000) that it is not credible to have a uranium enrichment in ${ }^{235} \mathrm{U}$ of greater than $50 \mathrm{wt} \%$ in this residue.

The evaluation shows that under the controls specified, assurance of subcriticality under normal and plausible upset conditions is provided which satisfies the double contingency criterion set forth in the Hanford Site Nuclear Criticality Safety Manuals. That is, at least two unlikely, independent, and concurrent changes in process conditions are required before a criticality is possible. 


\subsection{DOUBLE CONTINGENCY DOCUMENTATION}

This section presents a summary description of expected operations, expected normal conditions, and a base case summary of normal conditions plus anticipated off-normal conditions for storage of plutonium residue in $\mathbf{5 5}$ gallon drums. Plutonium residue consists of containers of sand, slag, and crucible (SS\&C), ash, oxides, and other miscellaneous residues from the cementation or pipe and go processes. These waste drums can be intermixed in storage with other waste drums that are also limited to $200 \mathrm{~g}$ of plutonium or fissile equivalent. In effect, this CSER also applies to the other waste drums provided the conditions and assumptions of this criticality safety evaluation are satisfied.

\section{Expected Operation}

Plutonium residue from SS\&C is cemented in Glovebox HA-20MB, bagged out, and placed into 55 gallon drums. Up to $200 \mathrm{~g}$ plutonium or fissile equivalent is placed either unconstrained in each drum or in a Pipe Overpack Container (POC) central tube arrangement. Ash, oxide, and miscellaneous residues are also expected to be loaded into these drums either in cement billets or directly packaged $(\mathrm{PnG})$ in a present storage form. These UN1A2 drums have nominal inner dimensions of $57.15 \mathrm{~cm}(22.5$ inches) and inner heights of $89.54 \mathrm{~cm}(35.25$ inches) and steel thickness of at least $0.15 \mathrm{~cm}(0.059$ inches) for the walls, bottom, and lids. Drums will have a rigid internal polyethylene liner that is $0.23 \mathrm{~cm}(0.09$ inches $)$ thick just on the inside of the drum wall, bottom, and top. Drums may also contain packing material and spider spacing arrangements.

Drums are transported to storage areas such as Rooms 172 and 192D in the 234-5Z building at the PFP. From here, they are moved to a loading dock area at the southwest corner of the building where they are loaded onto trucks and hauled to the Central Waste Complex or other storage locations.

\section{Expected Normal Conditions}

Waste material, described above, with up to $200 \mathrm{~g}$ of plutonium or fissile equivalent may be loaded into each 55 gallon drum along with packaging and packing material. The drums are transported one at a time from loading locations to storage locations where they are stored one tier high, in double rows (not a criticality limit), with walkways between. During transport, drums are spaced at least $45.7 \mathrm{~cm}$ (18 inches) from other fissile material, and stored at least 91.4 $\mathrm{cm}$ (36 inches) from such material as required by PFP transition and storage spacing requirements by CPS-Z-165-80100. A six-inch minimum spacing is allowed when moving past the north side of Glovebox HA-20 MB (Dobbin 2000).

\section{Base Case Summary}

The base case encompasses the normal and worst-case likely off-normal events. A calculation of an infinite by infinite by one array is a conservative representation of drum storage and movement of waste drums within an array. The array is modeled as a close-packed 
arrangement of standing cylinders, each with the optimum arrangement of $200 \mathrm{~g}$ of fissile material and optimum moderation and reflection as established in Section 5.2. The $\mathrm{k}_{\mathrm{eff}}$ for this case is $0.8392 \pm 0.0015$ (nonpipe refrotp). A more realistic base case is a $23^{\prime} \times 23^{\prime}, 12 \times 12$ drum, room-size array with $a k_{\text {eff }}=0.8357 \pm 0.0015$ (nonpipe_lat1). Storage area contingencies add the unlikely event to those base cases. To analyze drum transport from loading to storage, a drum is modeled adjacent to a wagon in a second alternate base case. A 5-position wagon loaded with $400 \mathrm{~g}$ of plutonium with unrestricted moderation per position is used to model the adjacent fissile material. The $k_{\text {eff }}$ for this case is $0.7593 \pm 0.0014$ (nonpipe5_wagon) and transport contingencies are added to this base case.

Table 1-1. Plutonium Residue in 55 Gallon Drums Base Case Summary

\begin{tabular}{|c|c|c|}
\hline Controlled Parameter & Limit & $\begin{array}{l}\text { Abnormal but Anticipated Conditions } \\
\text { and Conservatism for Analysis }\end{array}$ \\
\hline Mass & $\begin{array}{l}\text { Maximum } 200 \mathrm{~g} \mathrm{Pu} \\
\text { or fissile equivalent }\end{array}$ & $\mathrm{Pu}$ represented as $\mathrm{Pu}-239$ \\
\hline Volume & None & $\begin{array}{l}\text { Infinite array of drums for array calculation } \\
\text { when actually limited by room size }\end{array}$ \\
\hline Moderation & None & Optimized \\
\hline Interaction & $\begin{array}{l}\text { Drum arrays shall be } \\
\text { only one tier high; } \\
\text { minimum spacing to } \\
\text { other fissile material: } \\
\text { PFP transition and } \\
\text { storage spacing, } \\
\text { except other waste } \\
\text { drums not exceeding } \\
200 \mathrm{~g} \mathrm{Pu} \text {. }\end{array}$ & $\begin{array}{l}\text { Base case includes likely loss of spacing to } \\
\text { wagons during transport through } 234-5 Z \\
\text { Building hallways }\end{array}$ \\
\hline Reflection & None & $\begin{array}{l}\text { Optimized for filling of space within drums } \\
\text { with polyethylene }\end{array}$ \\
\hline Geometry & None & \\
\hline Enrichment & $\begin{array}{l}\text { Uranium enrichment } \\
\text { limited to } 50 \mathrm{wt} \%\end{array}$ & \\
\hline Density & None & Optimized for $200 \mathrm{~g}$ in 55 gallon drum \\
\hline Concentration & None & \\
\hline Poisons & None & $\begin{array}{l}\text { Steel in the drum reduced by } 50 \% \text { for } \\
\text { conservatism }\end{array}$ \\
\hline
\end{tabular}

The mass of fissile material in each container is established by nondestructive analysis (NDA) and carefully tracked to its placement into 55 gallon drums. Therefore, it is unlikely that 
the mass limits in the table above will be exceeded and subsequently no anticipated abnormal mass conditions are included in the box cases. If during the cementation process, a container with an NDA quantity of fissile material is split into more than one container, then all the resulting containers shall be placed into one 55 gallon drum to maintain an accurate account of the fissile mass.

\section{Contingency Summary}

Table 1-2 summarizes the analyses of the unlikely, independent, off-normal events (contingencies). Each event is assumed to occur with the drums configured with the most conservative base case with the contingency added. The resultant computed reactivity is compared to the subcriticality target $k_{\text {eff }}$ of 0.942 for MCNP calculations of plutonium systems similar to those in this evaluation, except for the cases involving plutonium billets where the subcriticality target $k_{\text {eff }}=0.932$. The destination between the benchmark analysis of plutonium metal versus plutonium in other forms is explained in Section 4.0. This table summarizes the results of the contingency evaluation found in Section 5.0. Specific $\mathrm{k}_{\mathrm{eff}}$ calculations were not made for contingencies that were bounded by other analyzed contingencies.

There is no contingency associated with the 50 weight percent U-235 enrichment limit because CSER 00-001 analysis (Dobbin 2000) for cementation operations determined that it is not credible to have cemented billets exceeding that limit.

Table 1-2. Plutonium Residue in 55 Gallon Drum Contingency Summary

\begin{tabular}{|c|c|c|c|}
\hline $\begin{array}{l}\text { Contingency } \\
\text { Description }\end{array}$ & $\begin{array}{l}\text { Affected } \\
\text { Parameter(s) }\end{array}$ & $\begin{array}{l}\text { Barriers that Make Contingency } \\
\text { Unlikely }\end{array}$ & $\begin{array}{l}\mathbf{k}_{\text {eff }} \text { Bounding } \\
\text { Contingency } \\
(\text { Case ID })^{1}\end{array}$ \\
\hline $\begin{array}{l}\text { Stacking of drums in } \\
\text { extra tier }\end{array}$ & Interaction & $\begin{array}{l}\text { Stacking is prohibited at PFP; no } \\
\text { mechanical device available to stack drums; } \\
\text { weight and size of drum would require two } \\
\text { operators to set one on another; entire } \\
\text { second tier stacked is unlikely }\end{array}$ & $\begin{array}{l}0.9264 \pm 0.0015 \\
\text { (nonpipe_refrot } 2 \ell)\end{array}$ \\
\hline Fissile mass overload & Mass & $\begin{array}{l}\text { Procedure; Operator training; material } \\
\text { inventory }\end{array}$ & $\begin{array}{l}0.7944 \pm 0.0014 \\
\text { (ovbtch_bot } 1 \mathrm{p} \text { ) for one } \\
\text { drum } 22 \% \text { overbatch; } \\
0.8947 \pm 0.0015 \\
\text { (ovbtch_refrotp) for entire } \\
\text { array } 22 \% \text { overbatched; } \\
0.8800 \pm 0.0017 \\
\text { (dblbtch_bot } 1 \mathrm{p} \text { ) for one } \\
\text { drum with } 400 \mathrm{~g} \text { Pu; } \\
0.9269 \pm 0.0017 \\
\text { (nonpipe_lat3) one drum } \\
\text { with } 400 \mathrm{~g} \text { in array }\end{array}$ \\
\hline $\begin{array}{l}\text { Overloaded wagon } \\
\text { next to drum }\end{array}$ & $\begin{array}{l}\text { Mass } \\
\text { Interaction }\end{array}$ & $\begin{array}{l}\text { Procedure; Operator training; spacing } \\
\text { required between drum and wagon }\end{array}$ & $\begin{array}{l}0.7941 \pm 0.0014 \\
\text { (nonpipe6_wagon) }\end{array}$ \\
\hline
\end{tabular}


HNF-6179 Rev. 0

\begin{tabular}{|l|l|l|l|}
\hline $\begin{array}{l}\text { Contingency } \\
\text { Description }\end{array}$ & $\begin{array}{l}\text { Affected } \\
\text { Parameter(s) }\end{array}$ & $\begin{array}{l}\text { Barriers that Make Contingency } \\
\text { Unlikely }\end{array}$ & $\begin{array}{l}\mathbf{k}_{\text {eff }} \text { Bounding } \\
\text { Contingency } \\
\text { (Case ID) }\end{array}$ \\
\hline $\begin{array}{l}\text { Overloaded drum next } \\
\text { to wagon }\end{array}$ & $\begin{array}{l}\text { Mass } \\
\text { Interaction }\end{array}$ & $\begin{array}{l}\text { Procedure; Operator training; spacing } \\
\text { required between drum and wagon }\end{array}$ & $\begin{array}{l}0.8919 \pm 0.0017 \\
\text { (nonpipe2_wagon) }\end{array}$ \\
\hline $\begin{array}{l}\text { Wagon next to 12 x } \\
12 \text { drum array }\end{array}$ & Interaction & $\begin{array}{l}\text { Procedure; Operator training; wagons not } \\
\text { likely in rooms with arrays of waste drums }\end{array}$ & $\begin{array}{l}0.8268 \pm 0.0015 \\
\text { (nonpipe_lat6) }\end{array}$ \\
\hline $\begin{array}{l}\text { Drums crushed by } \\
\text { concrete from floor } \\
\text { above or wall collapse }\end{array}$ & Geometry & Requires unlikely event such as earthquake & $\begin{array}{l}\text { Physical effect is the same } \\
\text { as second layer }\end{array}$ \\
\hline
\end{tabular}

\subsection{SUMMARY}

This criticality safety evaluation report (CSER) documents the criticality safety of storage of plutonium residues in 55 gallon drums with each drum containing up to $200 \mathrm{~g}$ plutonium or fissile equivalent. Some of the residues (i.e., SS\&C) will be treated for hazardous characteristics and stabilized by the cementation process in Glovebox HA-20MB. Direct packaging of residues without other stabilization activities, known as the Pipe-and-Go (PnG) alternative, has also been proposed for residues. It is expected that both processes will utilize the Pipe Overpack Container (POC). However, there may be cases when the UNIA2 galvanized drum will be used. This criticality safety evaluation includes loading, transport, and storage of the drums in the PFP 234-5Z building, excluding Room 227 , Room $230 \mathrm{C}$, and the vaults.

Evaluation of these billet drums included normal, base case, and contingencies. The base cases included both normal operation and the likely off-normal events, so that each contingency is evaluated upon the worst likely situation. A hazards analysis was conducted to assure that each credible unlikely event or set of correlated unlikely events was included in this analysis.

Demonstrating that each contingency has a calculated $\mathrm{k}_{\text {eff }}$ less than the subcritical safety limit shows that this operation meets the double contingency requirement. That is, at least two unlikely, independent, and concurrent changes in process conditions are required before a criticality is possible. Therefore, this CSER meets the requirements for a criticality evaluation contained in the Hanford Site Nuclear Criticality Safety Manuals, HNF-PRO-334 (FDH 2000), HNF-PRO-537 (FDH 1997a), and HNF-PRO-539 (FDH 1997b), ANSI/ANS-8 series Standards (ANSI 1998), and DOE Order 5480.24 (DOE 1992). This CSER also follows Fluor Federal Services, Inc. Practice 134.290.1121 (FFS 1999). 
HNF-6179 Rev. 0

This page intentionally left blank. 


\subsection{SYSTEM DESCRIPTION AND NORMAL OPERATIONS}

Plutonium residue will be mixed with cement to form billets or directly packaged and stored in 55 gallon drums in the 234-5Z Building at the PFP. The directly-packaged residue will use a PnG. With the exception of Room 227, Room 230C, and the vaults, the remainder of the 234-5Z Building can be used for loading and storage of these drums, as explained in the PHA in Appendix D. The drums will be shipped to another location to be prepared for ultimate disposal.

The following sections present the pertinent data necessary to evaluate the criticality safety of drum storage of this plutonium residue.

\subsection{PROCESS DESCRIPTIONS}

Plutonium bearing residues at PFP include Sand, Slag and Crucible residue (SS\&C), incinerator ash, impure Pu-oxides and other miscellaneous residues as described in CSER 00-001 (Dobbin 2000).

\section{Cementation}

SS\&C residues contain calcium metal and are mixed with water to react the calcium prior to mixing with cement and pouring into steel cans $14 \mathrm{~cm}$ in diameter and $17.8 \mathrm{~cm}$ high with a calculated volume of $2.725 \mathrm{~L}$ (designated in this report as $2.7 \mathrm{~L}$ containers to be consistent with how operations refer to these containers) to form billets that are allowed to harden, and stored in 55-gallon drums analyzed in this CSER. The billets will be formed in Glovebox HA-20MB with an operation that is described in CSER 00-001 (Dobbin 2000).

\section{Pipe and Go}

It is proposed that the pipe overpack container (POC) has the capability to accommodate selected residues. In the PnG process, residue is packaged and loaded directly into the POC. Ash is the only residue that is designated for packaging by the PnG method. However, utilization of this alternative for packaging other residue is being considered.

For both processes, each container is labeled with a mass that can be up to $200 \mathrm{~g}$ of plutonium or fissile equivalent. The plutonium and other fissile mass are assumed to be ${ }^{239} \mathrm{Pu}$. This simplification is conservative for the plutonium with more ${ }^{240} \mathrm{Pu}$ than ${ }^{241} \mathrm{Pu}$, which is the case for the reactor produced plutonium at PFP. The ${ }^{239} \mathrm{Pu}$ assumption also applies to the fissile atoms in depleted or natural uranium or mixed oxides of plutonium and uranium that may be present. For uranium, the ${ }^{235} \mathrm{U}$ enrichment (Marusich 1999) must not exceed $50 \mathrm{wt} \%$ in order for the ${ }^{239} \mathrm{Pu}$ to conservatively model this uranium. The carbon content of the feed material may vary. For the materials described in CSER 00-001, Appendices F and G, the carbon could be as high as $33 \%$ of the material. For other material, the carbon content is assumed to be $10 \%$, maximum. As described in Section 5.2, the moisture content of the cement waste was selected to provide the maximum reactivity for moderating material in billets in 55 gallon drums. 


\subsection{DRUM DESCRIPTION}

Cemented billet or residue cans are bagged out and placed into one of two types of 55 gallon drums. One has a rigid internal polyethylene liner that is $0.23 \mathrm{~cm}(0.09 \mathrm{in}$.) thick. The drums have an inner radius of $28.6 \mathrm{~cm}$, a wall thickness of $0.15 \mathrm{~cm}(0.06 \mathrm{in}$.) and an internal height of $84.5 \mathrm{~cm}$. The drum material is standard steel with a bulk density of $7.8 \mathrm{~g} / \mathrm{cc}$. Cans are added to the drum up to the drum limit for total plutonium or fissile equivalent mass. There is no specified geometry for these cans inside the drums. The second 55 gallon drum type, called a POC, has a central pipe extending the length of the drum and packing material to center the pipe in the drum.

\subsection{STORAGE ARRAY DESCRIPTION}

Drums will be loaded, transported, and stored as one-tier arrays in Building 234-5Z, except in Room 227, Room 230C, or the vaults. Normally, the drums are stored in double rows with a $91.6 \mathrm{~cm}$ walkway between each set of rows, but this is not a criticality safety requirement. Storage can be in rooms with concrete walls or of other construction. In Room 192D, FFTF fuel is stored on the other side of the concrete wall in Room 192A. In Room 172, there are several adjacent rooms in which there is fissile material. This evaluation will consider that during transport, the drum is likely to pass by wagons transporting fissile material. Also, the drums need to pass by other stored drums in the storage room, so an infinite array is used to model that storage. It is also desired to store drums right up to the wall next to Room 192A. Also, it is desired to intermix the storage of these drums with other waste drums that are limited to $200 \mathrm{~g}$ per drum.

The steel in the drums absorbs neutrons and tends to lower the $\mathrm{k}_{\mathrm{eff}}$ in this analysis. In order to be conservative and not require surveillance of the steel, the steel density is reduced by $50 \%$ in the calculations. It is not considered credible for these drums to have manufacturing tolerances that large or for corrosion or other natural phenomena to reduce the steel to less than half of the manufacturer's specifications. 


\subsection{LIMITS AND CONTROLS}

Table 3-1 lists each of the parameters of concern for criticality safety, and discusses whether these parameters are necessary.

Table 3-1. Controls on Parameters Related to Criticality Safety for Plutonium Billets in 55 Gallon Drums

\begin{tabular}{|l|c|l|}
\hline \multicolumn{1}{|c|}{ Parameter } & Controlled & \multicolumn{1}{|c|}{ Discussion (Limit or Process Control if Yes, Reason if No) } \\
\hline Mass & Yes & Maximum mass limit per drum. \\
\hline Volume & No & Optimum container volume used in analysis \\
\hline Spacing & Yes & $\begin{array}{l}\text { No drum stacking; PFP minimum transition and storage spacing } \\
\text { to other fissile material except other waste drums. }\end{array}$ \\
\hline Moderator & No & Optimum moderation in analysis. \\
\hline Reflector & No & Optimum neutron reflection used in analysis \\
\hline Poisons & No & Poisons were not used in this analysis. \\
\hline Concentration & No & Worst credible concentrations were analyzed. \\
\hline Enrichment & $\begin{array}{c}\text { Plutonium, } \\
\text { no; } \\
\text { Uranium, } \\
\text { yes }\end{array}$ & $\begin{array}{l}\text { Plutonium was assumed to be } 100 \text { wt\% } \%{ }^{239} \text { Pu. This } \\
\text { conservatively encompasses all allowed fissionable materials } \\
\text { including depleted or natural uranium and uranium enriched to } \\
50 \text { wt\% }{ }^{235} \text { U. }\end{array}$ \\
\hline Density & No & Optimized for 200 g of Pu in 55 gallon drum. \\
\hline Geometry & No & Worst-case geometry modeled. \\
\hline
\end{tabular}

\subsection{LIMITS}

Storage of fissile-containing cement billets in the 55-gallon drums is described in Section 2. Table 3-2 shows the parameter limits for storage using these drums.

Table 3-2. Limit Set for Storage of Plutonium Residue in 55 Gallon Drums

\begin{tabular}{|l|l|}
\hline Mass & Maximum $200 \mathrm{~g}$ plutonium or fissile equivalent per drum \\
\hline Volume & None \\
\hline Spacing & $\begin{array}{l}\text { Drum arrays shall be one tier high; minimum spacing to other } \\
\text { fissile material: } \mathrm{PFP} \text { transition and storage spacing, except other } \\
\text { waste drums not exceeding } 200 \mathrm{~g} \mathrm{Pu} .\end{array}$ \\
\hline Moderator & None \\
\hline Reflector & None \\
\hline
\end{tabular}


HNF-6179 Rev. 0

\begin{tabular}{|l|l|}
\hline Poisons & None \\
\hline Concentration & None \\
\hline Enrichment & $\begin{array}{l}\text { Plutonium not restricted; Uranium enrichment limited to } 50 \% \\
\text { 235 U. }\end{array}$ \\
\hline Density & None \\
\hline Geometry & None \\
\hline
\end{tabular}

\subsection{PROCESS CONTROLS}

To assure continued criticality safety during operations, the following process controls are required:

- Drums shall not be stacked.

- Firefighting category B.

- PFP transition and storage spacing requirements between array of these drums and other fissile material (CPS-Z-165-80100 1999) except waste drums.

- Split cementation batches must be placed in the same drum for fissile material accountability.

\subsection{ENGINEERED CONTROLS}

Engineered controls are the primary means of preventing a criticality for storage of plutonium residue in 55 gallon drums. These controls include the following:

- Structure of drum maintains separation of one drum from another.

- Concrete wall maintains $76.2 \mathrm{~cm}$ separation from these drums and FFTF fuel and $30.5 \mathrm{~cm}$ from pedestal or shelf stored fissile material.

\subsection{ADMINISTRATIVE CONTROL IMPLEMENTATION}

Criticality Prevention Specifications (CPS), postings and procedures provide limits and controls for handling fissionable materials, moderators, and other conditions that will assure criticality safety. These controls will address the limit set of Table 3-2 and process controls of Section 3.2.

The criticality analysis in this document demonstrates that a single failure of any administrative control will not result in a $k_{\text {eff }}$ that will exceed the criticality prevention criterion. 
HNF-6179 Rev. 0

\subsection{SUPPORTING INFORMATION}

Storage of plutonium residue in 55 gallon drums require:

Fire Fighting Category: B.

No restrictions on use of water for fire fighting is required. Intermixing these drums with waste drums is allowed. Waste drums were analyzed and reported in CSER 80-021 (Carter 1980 ) and fire fighting category B was required. In order to intermix, these drums will have the same designation.

\subsection{EVALUATION ASSUMPTIONS}

The important assumptions of this criticality safety evaluation are:

- Fissile material is conservatively modeled as Pu-239.

- It is not credible to have uranium with enrichment greater than 50\% U-235.

- Moderating material within billet is modeled to provide the greatest $\mathrm{k}$-effective for a drum array.

- In the cement model, calcium is replaced by silicon because the amounts of these two elements vary, depending upon the cement, and calcium is conservatively represented by silicon.

- Moderation optimized using polyethylene.

- The billets are loose within the drum and are in a triangular array with equal plutonium loading in each billet to provide the greatest $\mathrm{k}_{\mathrm{efr}}$ as established in CSER 96-027 (Watson 1997).

- None of the calculations accounted for the iodine constituent of the real SS\&C residues, a conservative omission because of the high neutron absorption properties for the element ( 7 barns at thermal energies, a factor of 20 times the absorption for hydrogen).

- For the cement mix, iron oxide, as sometimes present in concrete, was excluded.

- Drums are excluded from Room 227, Room 230C, and the vaults in Building 234-5Z.

- It is not credible for the drums to have less than half of the $24 \mathrm{~kg}$ of steel established by their specifications. Calculations include $12 \mathrm{~kg}$ of steel per drum, which acts as a neutron absorber in this lattice arrangement. 


\section{HNF-6179 Rev. 0}

- A fully loaded wagon adjacent to drum can create a higher $\mathrm{k}_{\mathrm{eff}}$ than any other fissile item encountered in Building 234-5Z. 


\subsection{METHODOLOGY}

\subsection{ANALYSIS PHILOSOPHY}

The computer code, MCNP (Briesmeister 1997), was used to calculate the k-effective of the allowed limit sets and credible upset events. Validation of this code was performed by evaluation of benchmark experiments involving the materials that will be used in this glovebox. As described in Section 4.2, Erickson evaluated plutonium and uranium in systems involving water and concrete as reflectors and water as a moderator. Because the drums contain billets of cement mixed with the fissile material, another benchmark experiment using concrete as a moderator was evaluated as reported in Appendix B of CSER 00-001 (Dobbin 2000).

Nominal dimensions and optimum moderation are used throughout the analyses. Where appropriate, minimum spacing between fissile masses is modeled for conservatism. Only mass and spacing parameters require administrative control for this drum storage. It is not credible to have any uranium enriched to more than $50 \%{ }^{235} \mathrm{U}$.

\subsection{MCNP CODE}

The Monte Carlo code MCNP Version 4B, was certified (Schwinkendorf 1998) and validated (Erickson 1998 and Lan 1999) for plutonium systems such as the operation in this glovebox. Because that validation did not include concrete or cement as a moderator, an additional validation was performed and reported in CSER 00-001 Appendix B, MCNP 4B Computer Code Validation. Room temperature cross sections were used for the MCNP calculations. In Appendix B, a maximum allowable $k_{\text {eff }}$ value of 0.932 was established for calculations of systems dominated by large metal pieces such as plutonium buttons. For this cementation process, the oxide maximum allowable $\mathrm{k}_{\mathrm{eff}}$ of 0.942 is more appropriate because it more closely matches the benchmarks of lower density fissile material. These values are for calculations with statistical uncertainties $\leq 0.002$ and assure subcriticality with an acceptable margin, including the uncertainties in the analytical methods and benchmark experimental data. The oxide target $\mathrm{k}_{\text {eff }}$ is applicable to other plutonium compounds listed in Greenborg 1999, and uranium up to an enrichment of $50 \%{ }^{235} \mathrm{U}$ (Marusich 1999).

\subsection{SUBCRITICALITY LIMIT}

For the purposes of this report, the principal criticality prevention criterion or parameter is that the effective neutron multiplication (or criticality) factor $\left(\mathrm{k}_{\text {eff }}\right)$ shall not exceed 0.95 (i.e., $k_{\text {eff }} \leq 0.95$ ) for all permitted normal configurations of materials, containers, etc., and for any credible off-normal event. This criterion is based on implementing the applicable DOE Orders, ANSI standards, and HNF-PROs. The subcriticality criterion is used to judge the acceptability of a calculated $k_{\text {eff }}$ value for fissionable material configuration. This criterion accounts for the bias inherent in the code and cross sections used, any uncertainties in the physical problem being MCNP and Monte Carlo are computer codes developed by Los Alamos National Laboratory. 
analyzed, and the uncertainties in both the bias determination (the experimental basis) and the calculational methods.

\subsection{APPLICATION OF DOUBLE CONTINGENCY PRINCIPLE}

This analysis meets the requirements of HNF-PRO-334, Criticality Safety General Requirements, (FDH 2000), HNF-PRO-537, Criticality Safety Control of Fissionable Material, (FDH 1997a) and HNF-PRO-539, Criticality Safety Evaluations (FDH 1997b). HNF-PRO-539 states that for all new operations and changes pertinent to criticality safety issues in existing operations, the CSER is required to demonstrate that there is an acceptable margin of subcriticality for all normal and credible abnormal conditions. To demonstrate the Double Contingency Principle is satisfied, this CSER must show that there are sufficient factors of safety for plutonium billet storage in 55 gallon drums such that at least two unlikely, independent, and concurrent changes in process conditions are required before a criticality accident is possible.

\subsection{HAZARDS ASSESSMENT}

Identification of the contingencies for the operation described in Section 2 and evaluated in Section 5 used a hazards assessment technique called a Preliminary Hazard Assessment (PHA). The goal of this effort is to identify deviations from the planned operation that may pose a challenge to criticality safety. Analysis is done as necessary to demonstrate that each identified condition satisfies the criticality safety criteria.

In a PHA, an interdisciplinary team uses a disciplined, systematic approach to identify hazards and deviations that could lead to undesirable consequences. Because the criticality safety concerns usually arise from deviations from the process design, an experienced team leader systematically guides the team through the planned operation. Off-normal events are identified and separated into likely and unlikely to happen during the duration of the operation. The likely events become part of the base case and the unlikely events are the contingencies of Section 5. The detailed results of this assessment are presented in Appendix D. 


\subsection{EVALUATION AND RESULTS}

\subsection{NORMAL CASE}

Drums are loaded one at a time and transported to one of the storage rooms where they are arranged in double rows with walkways between sets of these double rows. Drums will be transported down these walkways between the rows. Therefore, the value of a calculation of the normal array is questionable. For this evaluation, a normal case calculation is bypassed for the conservative base case of an infinite by infinite by one tier high array calculation described in the next section.

\subsection{BASE CASE}

This section presents the base case models of the plutonium billets in 55 gallon drums. The results of CSER 96-027 (Watson 1997) were utilized to ascertain the most reactive configuration of billets within the drum. The case where the $200 \mathrm{~g}$ allowed in the drum was distributed equally between three billets in a triangular array proved to be the most reactive. For this evaluation, the triangular billet array is located near the edge of the drum adjacent to fissile material either in an adjacent drum or in an adjacent wagon.

The mass of fissile material in each container is established by nondestructive analysis (NDA) and carefully tracked until placed into 55 gallon drums. Therefore, it is unlikely that the mass limits in the table above will be exceeded and subsequently no anticipated abnormal mass conditions are included in the base cases. If during the cementation process, a container with an NDA quantity of fissile material is split into more than one container, then all the resulting containers shall be placed into one 55 gallon drum to maintain an accurate account of the fissile mass.

Cemented containers were modeled as cylinders with a volume of $2.725 \mathrm{~L}$ with interior diameter of $14 \mathrm{~cm}$ and a height of $17.8 \mathrm{~cm}$. For mixtures of fissile material and cement, the cement acts as a moderator and the most reactive container is one with the most water (Dobbin 2000). So, a mixture of plutonium and water was modeled in each billet container. Drums were modeled as described in Section 2.2. Material densities and weight fractions used in this analysis are shown in Table 5-1.

Infinite arrays, finite room-sized arrays, and single drums were analyzed. Infinite arrays are simple calculations that bound the worst $\mathrm{k}_{\text {eff }}$ possible from assembling drums. A finite array is required to analyze the contingency of violating the PFP transition and storage minimum spacing requirement. For this evaluation, a transport wagon is modeled adjacent to the room array of drums. A single drum allows other fissile material, that may be encountered in transit within the PFP 234-5Z Building, to be modeled adjacent to the drum. 
HNF-6179 Rev. 0

Table 5-1. Elemental Weight Fractions and Densities of Materials used in Calculations

\begin{tabular}{|l|l|l|l|l|l|l|l|}
\hline & $\begin{array}{l}\text { Cemented } \\
\text { Waste } \\
\mathbf{1 . 0 2 4} \\
\mathbf{g} / \mathbf{c m}^{\mathbf{3}}\end{array}$ & $\begin{array}{l}\text { Steel } \\
\mathbf{7 . 8 3} \mathbf{g} / \mathbf{c m}^{\mathbf{3}}\end{array}$ & $\begin{array}{l}\text { Air } \\
\mathbf{0 . 0 0 1 2 9} \\
\mathbf{g} / \mathbf{c m}^{\mathbf{3}}\end{array}$ & $\begin{array}{l}\text { Polyethylene } \\
\mathbf{0 . 9 2} \mathbf{g} / \mathbf{c m}^{\mathbf{3}}\end{array}$ & $\begin{array}{l}\text { Concrete } \\
\mathbf{2 . 2 4} \\
\mathbf{g} / \mathbf{c m}^{\mathbf{3}}\end{array}$ & $\begin{array}{l}\text { Water } \\
\mathbf{1 . 0} \\
\mathbf{g} / \mathbf{c m}^{\mathbf{3}}\end{array}$ & $\begin{array}{l}\text { Cemented Waste } \\
\text { with 45 wt\% } \\
\text { Water } \\
\mathbf{1 . 5 1} \mathbf{g} / \mathbf{c m}^{\mathbf{3}}\end{array}$ \\
\hline $\mathrm{H}$ & 0.1092 & & & 0.143702 & 0.009959 & 0.1119 & 0.0632 \\
\hline $\mathrm{C}$ & & 0.000293 & & 0.856298 & 0.000979 & & \\
\hline $\mathrm{N}$ & & & 0.754918 & & & & \\
\hline $\mathrm{O}$ & 0.8669 & & 0.231967 & & 0.529069 & 0.8881 & 0.6211 \\
\hline $\mathrm{F}$ & & & & & & & \\
\hline $\mathrm{Na}$ & & & & & 0.016003 & & \\
\hline $\mathrm{Mg}$ & & & & & 0.002000 & & \\
\hline $\mathrm{Al}$ & & & & & 0.034006 & & \\
\hline $\mathrm{Si}$ & & 0.007497 & & & 0.337036 & & 0.2995 \\
\hline $\mathrm{P}$ & & 0.000447 & & & & & \\
\hline $\mathrm{S}$ & & 0.000294 & & & & & \\
\hline $\mathrm{Ar}$ & & & 0.013115 & & & & \\
\hline $\mathrm{K}$ & & & & & 0.012981 & & \\
\hline $\mathrm{Ca}$ & & & & & 0.043965 & & \\
\hline $\mathrm{Cr}$ & & 0.200003 & & & & & \\
\hline $\mathrm{Mn}$ & & 0.020000 & & & & & \\
\hline $\mathrm{Fe}$ & & 0.651464 & & & 0.014002 & & \\
\hline $\mathrm{Ni}$ & & 0.120002 & & & & & \\
\hline $\mathrm{Pu}$ & 0.0239 & & & & & & 0.0162 \\
\hline
\end{tabular}

* For $100 \%$ free water in cement.

There is also a possibility that plastic "peanuts" will be packed in the drums with loose billets. Thus the effect of a polyethylene reflector within the drum was investigated with the free water content of the cement at an arbitrary $45 \mathrm{wt} \%$. Figure 2 illustrates that full density polyethylene (Carter 1968) proved to be the most effective moderator/reflector, and the rest of the cases were run with this value. For billets modeled as plutonium and water, the full density polyethylene is still the most effective moderator/reflector. The curves are for one drum, two drums together, and an infinite array of drums. This is a very conservative assumption since the peanuts will have a much higher void fraction than solid polyethylene. 
Figure 1: $k_{\text {eff }}$ for $200 \mathrm{~g}$ in Three Billets in a 55 Gallon Drum Versus Density of Polyethylene Fill.

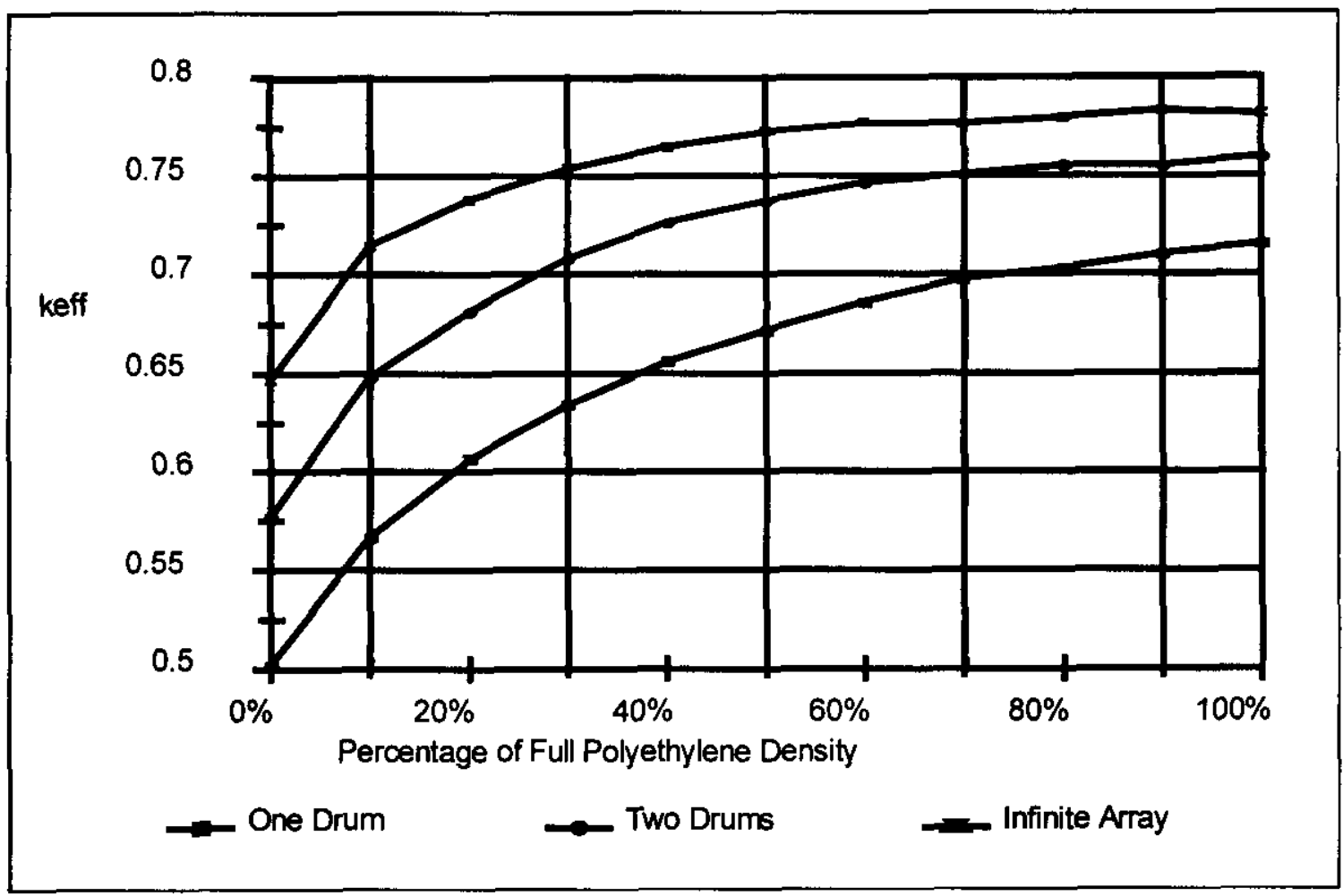

Since these drums can be intermixed with standard waste drums, the reactivity of a single drum was compared with the most reactive drum analyzed in the CSER for solid fissile material storage in 55-gallon drums (Carter 1980). The model has $200 \mathrm{~g}$ of plutonium in the shape of a cylinder $(\mathrm{H} / \mathrm{D}=1.5)$ in a matrix of polyethylene at the most reactive concentration $(\mathrm{H} / \mathrm{Pu}=730)$. The $\mathrm{k}_{\text {eff }}$ of a drum with cemented waste was $0.7733 \pm 0.0013$ (nonpipe_cp), while the $\mathrm{k}_{\text {eff }}$ of the generic waste drum was $0.4768 \pm 0.0010$ (cement/odrum_bot 1 ). Thus the addition of other currently approved waste packed in 55-gallon drums will be less reactive than the cemented waste in 55-gallon drums.

In all of the calculations the billets are assumed to be unrestricted within the drum. The use of the pipe overpack drum, which constrains the fissile material to a central pipe region, will be a less reactive situation and so the unrestricted arrangement was used as a limiting case. The base case calculations are presented in the sections below. Details of the base models are summarized in Tables 5-1, 5-2, and 5-4. Results are presented in Tables 5-3 and 5-5.

For all cases the plutonium involved was assumed to be entirely ${ }^{239} \mathrm{Pu}$. Also, none of the calculations accounted for the iodine constituent of the real SS\&C residues, a conservative omission because of the high neutron absorption properties for the element ( 7 barns at thermal energies, a factor of 20 times the absorption for hydrogen). For the cement mix, iron oxide, as sometimes present in concrete, was excluded. 


\subsubsection{Infinite Array of Drums}

The base case model for an infinite by infinite by one tier high array of drums has a $\mathrm{k}_{\mathrm{eff}}$ of $0.8357 \pm 0.0015$ (case nonpipe lat 1 ). This value is well below the allowable $k_{\text {eff }}$ of 0.942 .

Description of this model is as follows.

Table 5-2. Base Case Model for Infinite Array

\begin{tabular}{|l|l|}
\hline Drum Array Assumptions & $12 \times 12 \times 1$ or $\infty \times \times 1$ \\
\hline Drum Dimensions & $\begin{array}{l}\text { Inner Radius }=28.575 \mathrm{~cm} \\
\text { Height }=83.998 \mathrm{~cm} \\
\text { Wall thickness }=0.242 \mathrm{~cm}\end{array}$ \\
\hline Billet cans & $\begin{array}{l}\text { Inner Radius }=6.985 \mathrm{~cm} \\
\text { Height }=17.780 \mathrm{~cm} \\
\text { Vol }=2.725 \mathrm{~L}\end{array}$ \\
\hline
\end{tabular}

Table 5-3. MCNP Calculational Results of Base Case of Infinite Array of Drums

\begin{tabular}{|c|l|c|c|}
\hline Case & \multicolumn{1}{|c|}{ Description } & \multicolumn{1}{|c|}{$\mathbf{k}_{\text {calc }}$} & $1 \sigma$ \\
\hline $\begin{array}{c}\text { Nonpipe } \\
\text { latl }\end{array}$ & $\begin{array}{l}12 \times 12 \times 1 \text { array of drums with } 200 \mathrm{~g} \mathrm{Pu} / \text { drum distributed in } \\
\text { three billets }\end{array}$ & 0.8357 & 0.0015 \\
\hline $\begin{array}{c}\text { Nonpipe__ } \\
\text { refrotp }\end{array}$ & $\begin{array}{l}\infty \times \infty \times 1 \text { array of drums with } 200 \mathrm{~g} \mathrm{Pu} / \text { drum distributed in } \\
\text { three billets }\end{array}$ & 0.8392 & 0.0015 \\
\hline
\end{tabular}

\subsubsection{Drum with Wagon}

The base case model for a drum and wagon with $2.5 \mathrm{~kg}$ of metal in each of the wagon's five positions has $a \mathbf{k}_{\text {eff }}$ of $0.7593 \pm 0.0014$ (nonpipe5_wagon). This value is well below the allowable $\mathrm{k}_{\text {eff }}$ for a system with metal buttons of 0.932 . Description of this model is presented in Table 5-4. This is the most reactive of the following three wagon loadings:

- $4.5 \mathrm{~kg}$ of dry plutonium oxide

- $400 \mathrm{~g}$ of plutonium in a can full of water $(2.7 \mathrm{~L})$

- $2.5 \mathrm{~kg}$ of metal

A combination of these loadings was not calculated because CSER 87-004 (Ramble 1999 ) states that intermixing of the $2.5 \mathrm{~kg}$ metal and oxide limit sets does not increase the $\mathrm{k}_{\text {eff }}$ above the all-metal case. There is no reason to believe that the presence of an adjacent drum would alter this conclusion because CSER 87-004 also had adjacent wagons.

Present wagon CSERs do not allow the $4.5 \mathrm{~kg}$ of dry plutonium oxide. When the evaluation to alter the higher mass in wagons is performed, the intermixing will again be 
addressed. If intermixing the higher fissile mass is found to increase $\mathrm{k}_{\mathrm{eff}}$, then this CSER will need to be reviewed prior to allowing the higher mass in the wagons.

Table 5-4. Base Case Model for Drum Next to Wagon

\begin{tabular}{|l|l|}
\hline Drum Dimensions & $\begin{array}{l}\text { Inner Radius }=28.575 \mathrm{~cm} \\
\text { Height }=83.998 \mathrm{~cm} \\
\text { Wall thickness }=0.242 \mathrm{~cm}\end{array}$ \\
\hline Billet cans & $\begin{array}{l}\text { Inner Radius }=6.985 \mathrm{~cm} \\
\text { Height }=17.780 \mathrm{~cm} \\
\text { Vol }=2.725 \mathrm{~L}\end{array}$ \\
\hline Wagon Assumptions & $\begin{array}{l}\text { A five position wagon was used with a } 2.7 \text { L can at each of the five } \\
\text { positions. Each can is filled with the plutonium and moderation } \\
\text { described in Table 5-5. The wagon is completely surrounded by } \\
\text { full density polyethylene. }\end{array}$ \\
\hline
\end{tabular}

Table 5-5. MCNP Calculational Results of Base Case of a Drum Next to a Wagon

\begin{tabular}{|c|l|c|c|}
\hline Case & \multicolumn{1}{|c|}{ Description } & $\mathbf{k}_{\text {calc }}$ & $1 \sigma$ \\
\hline $\begin{array}{c}\text { Nonpipe3_- } \\
\text { wagon }\end{array}$ & $\begin{array}{l}\text { Wagon } \\
\text { One drum with three unrestricted moderation billets for a } \\
\text { total of 200 } \mathrm{g} \text { Pu in a triangular array in intimate contact with } \\
\text { the center of the three position side of a five position wagon } \\
\text { with 400 g Pu unrestricted moderation in each position. The } \\
\text { fissile material in the drum is raised to be in the same plane } \\
\text { as the fissile material in the wagon. }\end{array}$ & 0.7507 & 0.0016 \\
\hline $\begin{array}{c}\text { Nonpipe_- } \\
\text { wagon }\end{array}$ & $\begin{array}{l}\text { Same as nonpipe3_wagon except 4.5 kg Pu oxide at } \\
\text { theoretical density in each wagon position. }\end{array}$ & 0.7558 & 0.0014 \\
\hline $\begin{array}{c}\text { Nonpipe5_- } \\
\text { wagon }\end{array}$ & $\begin{array}{l}\text { Same as nonpipe3_wagon except 2.5 kg Pu metal at } \\
\text { theoretical density in each wagon position }\end{array}$ & 0.7593 & 0.0014 \\
\hline
\end{tabular}

\subsubsection{Interaction of Array of Drums with Material in Adjacent Rooms}

Fissionable material is stored in rooms adjacent to those in which the drums with cemented waste may be stored. If the drums are stored in room 192-D, the adjacent room is 192-A in which is stored FFTF fuel assemblies. However, the two rooms are separated by a $76.2 \mathrm{~cm}(2.5 \mathrm{ft})$ concrete wall which will isolate the fissile material in 192-A from the drums in 192-D.

Room 172, another possible location for the storage of the cemented drums, is adjacent to rooms 173,175 , and 179 , and shares a corner with room 174 . Rooms 173, 174, and 175 are separated from 172 by $30.5 \mathrm{~cm}$ (1 ft) concrete walls. Room 174 is a vault for shelf storage of fissile material in containers with up to $4.4 \mathrm{~kg}$ of material inside. Room 175 is a vault for 
pedestal storage for similar containers. Room 173 is also a vault, but no fissile material is stored there. The concrete wall will isolate the material in these vaults from the cemented waste drums.

Any approved waste drum stored in adjacent rooms has been shown to be less reactive than the cemented waste container (see page 5-3). Other fissile material containers may be more reactive than a waste drum, therefore, PFP transfer and spacing requirements require a $91.4 \mathrm{~cm}$ ( $3 \mathrm{ft}$ ) spacing between an array of cemented drums and other fissile material.

\subsection{CONTINGENCY ANALYSIS}

The contingency analysis section addresses the effect of various unlikely, off-normal events on the critical parameters and their associated controls to confirm the double contingency criterion has been met.

Mass is controlled administratively. Section 5.3.4 addresses the contingency of exceeding the drum limit of $200 \mathrm{~g}$ plutonium or equivalent.

Volume and Geometry are not controlled.

Density is not controlled. The most reactive credible densities were used for all materials.

Moderation is not controlled.

Reflection is not controlled.

Interaction is controlled by a no drum stacking requirement, the general transportation and storage spacing requirements, and isolating walls. Sections 5.3.5 and 5.3.6 address interaction contingencies.

Enrichment, Concentration, and Isotopes are not controlled for plutonium which was assumed to be $100 \mathrm{wt} \%{ }^{239} \mathrm{Pu}$. This conservatively encompasses all allowed fissionable materials including depleted or natural uranium and uranium enrichment which is restricted to $50 \mathrm{wt} \%{ }^{235} \mathrm{U}$. The hazards assessment of CSER 00-001 (Dobbin 2000) states that it isn't credible for this residue to exceed that uranium enrichment.

Neutron Absorption is not controlled.

The following discussions in this section give a description of the off-normal conditions and the calculational results. Each of the unlikely off-normal events results from a loss of one or more controls, and is therefore considered to be a contingency. For each contingency, the model assumed the most limiting allowed conditions for criticality controls shown in Section 3 including likely off-normal events. 


\subsubsection{Seismic Event}

Three dimensional collapse was judged by the HAZOP process to be incredible. However, in a seismic event the drum could be crushed in the vertical dimension. However, this contingency is not limiting for the following reasons.

The drums have been analyzed in their most reactive close packed arrangement. Any disturbance due to a seismic event will tend to lower the reactivity. A one dimensional seismic induced collapse of the building will tend to crush the drums, but would not compress the billets. With the billets in their most reactive arrangement, fully reflected in all directions, no substantial change in conditions would result from the seismic event. In fact, the two layer array scenario would encompass the seismic scenario since the billets in the lower layer were modeled at the top of the drum while the billets in the upper layer were modeled on the bottom. Thus there are two layers of nine billets each in the most intimate contact possible. The analysis has considered billets in the most reactive arrangement and these contingencies would not result in a more reactive condition. The POC drums will have additional structural integrity provided by the vertical pipe. The analysis has considered maximum polyethylene interspersed moderation and reflection. Therefore, the addition of water between the drums due to a breaching of the fire suppressant system would not add additional reactivity.

\subsubsection{Major Fire}

The analysis has considered maximum polyethylene reflection. The addition of water between the drums due to an activation of the fire suppressant system would not add additional reactivity.

\subsubsection{Stacking Event}

The addition of a complete second layer of loaded drums was considered as described in Table 5-6. Both of the layers have the most reactive geometry, water content, and polyethylene reflector. This contingency case model for a double array of drums has a $\mathrm{k}_{\text {eff }}$ of $0.9264 \pm 0.0015$ (nonpipe_refrot2l), listed in Table 5-7. This value is below the allowable $\mathrm{k}_{\text {eff }}$ of 0.942 . A calculation with an array of drums filling a $700 \mathrm{~cm}$ by $700 \mathrm{~cm}\left(23^{\prime} \times 23^{\prime}\right)$ room with concrete walls with three drums stacked in a second layer produced a $k_{\text {eff }}$ of $0.8368 \pm 0.0013$ (nonpipe_lat4). 
Table 5-6. Contingency Case Model for Two Layer Arrays

\begin{tabular}{|l|l|}
\hline Drum Array Assumptions & $\infty \times \infty \times 2$ and $12 \times 12 \times 1$ \\
\hline Drum Dimensions & $\begin{array}{l}\text { Inner Radius }=28.575 \mathrm{~cm} \\
\text { Height }=83.998 \mathrm{~cm} \\
\text { Wall thickness }=0.242 \mathrm{~cm}\end{array}$ \\
\hline Billet cans & $\begin{array}{l}\text { Inner Radius }=6.985 \mathrm{~cm} \\
\text { Height }=17.780 \mathrm{~cm} \\
\text { Vol }=2.725 \mathrm{~L}\end{array}$ \\
\hline
\end{tabular}

Table 5-7. Calculational Results for Two Layer Arrays

\begin{tabular}{|l|l|c|c|}
\hline \multicolumn{1}{|c|}{ Case } & \multicolumn{1}{|c|}{ Description } & $\mathbf{k}_{\text {calc }}$ & $1 \sigma$ \\
\hline $\begin{array}{l}\text { Nonpipe_ } \\
\text { refrot21 }\end{array}$ & Two infinite layers of drums & 0.9264 & 0.0015 \\
\hline Nonpipe_lat4 & $\begin{array}{l}\text { One 700 cm x 700 cm lower layer of drums with three } \\
\text { drums stacked in second layer within a concrete box }\end{array}$ & 0.8368 & 0.0013 \\
\hline
\end{tabular}

\subsubsection{Overbatching}

The overbatching of the drums was considered. Considering Safeguards limitations (Watson 1997), the cemented waste may have up to $2 \mathrm{wt} \%$ of plutonium. However, if three billets of such material are loaded into a drum, the $200 \mathrm{~g}$ limit on the drum will be exceeded. It was assumed that the most probable overbatch event is for three of these billets to be inadvertently loaded into a drum. Therefore, this contingency could result in an infinite layer of drums loaded with three billets of $2 \mathrm{wt} \%$ plutonium or a total of $244 \mathrm{~g}$ of plutonium in each drum as described in Table 5-8. This event is extremely unlikely. The drums are assumed to have the most reactive geometry, water content, and polyethylene reflector.

In Appendix D, it was considered credible to have $10 \%$ of the drums overbatched by $10 \%$ or have one drum with $400 \mathrm{~g}$ plutonium. Table 5-9 shows the calculated $\mathrm{k}_{\text {eff }}$ 's of drum overbatches. For a single drum double-batched, $\mathrm{k}_{\text {eff }}=0.8800 \pm 0.0017$ (dblbtch_bot $\left.1 \mathrm{p}\right)$. For an entire array of drums containing $244 \mathrm{~g}$ plutonium, $\mathrm{k}_{\mathrm{eff}}=0.8947 \pm 0.0015$ (ovbtch_refrotp). For a $400 \mathrm{~g}$ plutonium drum in an array of $200 \mathrm{~g}$ plutonium drums, $\mathrm{k}_{\mathrm{eff}}=0.9269 \pm 0.0017$ (nonpipe_lat3). These values are below the allowable $\mathrm{k}_{\mathrm{eff}}$ of 0.942 . 
HNF-6179 Rev. 0

Table 5-8. Contingency Case Model for Overbatching

\begin{tabular}{|l|l|}
\hline Drum Array Assumptions & $\infty \times \infty \times 1$ and $12 \times 12 \times 1$ \\
\hline Drum Dimensions & $\begin{array}{l}\text { Inner Radius }=28.575 \mathrm{~cm} \\
\text { Height }=83.998 \mathrm{~cm} \\
\text { Wall thickness }=0.242 \mathrm{~cm}\end{array}$ \\
\hline Billet cans & $\begin{array}{l}\text { Height }=17.780 \mathrm{~cm} \\
\text { Vol }=2.725 \mathrm{~L} \\
\text { Inner Radius }=6.985 \mathrm{~cm}\end{array}$ \\
\hline Cemented Waste & \begin{tabular}{l} 
wt\% plutonium in cement $(244 \mathrm{~g} / \mathrm{drum})$ \\
\hline
\end{tabular} \\
\hline
\end{tabular}

Table 5-9. Calculational Results for Overbatching

\begin{tabular}{|l|l|c|c|}
\hline \multicolumn{1}{|c|}{ Case } & \multicolumn{1}{|c|}{ Description } & $\mathbf{k}_{\text {calc }}$ & $1 \sigma$ \\
\hline Ovbtch_bot1p & One drum with 244 g Pu & 0.7944 & 0.0014 \\
\hline Ovbtch_ref2p & Two drums each with 244 g Pu & 0.8508 & 0.0015 \\
\hline Ovbtch_refp & Infinite array with fissile material facing in pairs of drums & 0.8786 & 0.0014 \\
\hline Ovbtch_refrotp & Infinite array with fissile material facing in triads of drums & 0.8947 & 0.0015 \\
\hline Dblbtch_botlp & One drum with 400 g Pu & 0.8800 & 0.0017 \\
\hline Nonpipe_lat3 & One drum with 400 g Pu in 200 g Pu per room drum array & 0.9269 & 0.0017 \\
\hline
\end{tabular}

\subsubsection{Interactions with Other Fissile Material}

Of the fissile material available at the PFP that could inadvertently be brought next to an array of billet drums, the wagon analyzed in Section 5.2.2 would have the greatest reactivity impact. A wagon with 5 containers each containing various allowed loadings of fissile material and moderation are analyzed adjacent to a normal and overloaded drum and adjacent to an array of drums as described in Table 5-10. Also, an overloaded wagon is analyzed next to a normal drum. Results are shown in table 5-11. The worst overloaded drum, normal wagon arrangement has a $k_{\text {eff }}$ of $0.8919 \pm 0.0017$ (nonpipe4_wagon), listed in Table 5-11 which is lower than the allowable $\mathrm{k}_{\text {eff }}$ of 0.942 . The worst overloaded wagon, normal drum arrangement has a $\mathrm{k}_{\text {eff }}$ of $0.7941 \pm 0.0014$ (nonpipe6_wagon) which is lower than the allowable $k_{\text {eff }}$ of 0.932 for metal systems. For a wagon next to an array of drums, that $k_{\text {eff }}$ of $0.8268 \pm 0.0015$ (nonpipe_lat6) is lower than the allowable $\mathrm{k}_{\mathrm{eff}}$ of 0.932 for metal systems. 
Table 5-10. Contingency Case Model for Wagon Next to Single Drum or Array

\begin{tabular}{|l|l|}
\hline Drum Array Assumptions & $12 \times 12 \times 1$ and $1 \times 1 \times 1$ \\
\hline Drum Dimensions & $\begin{array}{l}\text { Inner Radius }=28.575 \mathrm{~cm} \\
\text { Height }=83.998 \mathrm{~cm} \\
\text { Wall thickness }=0.242 \mathrm{~cm}\end{array}$ \\
\hline Billet cans & $\begin{array}{l}\text { Inner Radius }=6.985 \mathrm{~cm} \\
\text { Height }=17.780 \mathrm{~cm} \\
\text { Vol }=2.725 \mathrm{~L}\end{array}$ \\
\hline Wagon Assumptions & $\begin{array}{l}\text { A five position wagon was used with a 2.7 L can in each of the } \\
\text { five positions. Each can is filled with either } 400 \mathrm{~g} \text { Pu with } \\
\text { unrestricted moderation, } 2.5 \mathrm{~kg} \text { of Pu metal, or } 4.5 \mathrm{~kg} \text { of plutonium } \\
\text { oxide at theoretical density. The wagon is completely surrounded } \\
\text { by full density polyethylene. }\end{array}$ \\
\hline
\end{tabular}

Table 5-11. Calculational Results for Wagon Next to Single Drum or Array

\begin{tabular}{|l|l|c|c|}
\hline \multicolumn{1}{|c|}{ Case } & \multicolumn{1}{|c|}{ Description } & \multicolumn{1}{|c|}{$\mathbf{k}_{\text {calc }}$} & $1 \sigma$ \\
\hline Nonpipe4_wagon & wagon with 4.5 kg Pu except 400 g Pu in drum & 0.8919 & 0.0017 \\
\hline Nonpipe2_wagon & Same as nonpipe3_wagon except 400 g Pu in drum & 0.8899 & 0.0018 \\
\hline Nonpipe_lat5 & Wagon with 4.5 kg oxide adjacent to array of drums & 0.8244 & 0.0015 \\
\hline Nonpipe_lat6 & $\begin{array}{l}\text { Wagon with 2.5 kg Pu metal adjacent to an array of } \\
\text { drums }\end{array}$ & 0.8268 & 0.0015 \\
\hline Nonpipe6_wagon & $\begin{array}{l}\text { Overloaded wagon of 2.5 kg Pu metal adjacent to single } \\
\text { drum }\end{array}$ & 0.7941 & 0.0014 \\
\hline
\end{tabular}




\subsection{REFERENCES}

ANSI/ANS-8.1-1983: R1998, Nuclear Criticality Safety in Operations with Fissionable Materials Outside Reactors, American Nuclear Society, La Grange Park, Illinois.

Briesmeister, J.F., 1997, MCNP - A General Monte Carlo N-Particle Transport Code, Version $4 B$, LA-12625-M, Los Alamos National Laboratory, Los Alamos, New Mexico

Carter, R. D., 1980, CSAR 80-021, Packaging and Disposal of Solid Waste (55-Gallon Drums, Unrestricted H/Pu Only), Rockwell Hanford Operations, Richland, Washington.

Carter, R. D., G. R. Kiel, and K. R. Ridgway, 1968, Criticality Handbook, ARH-600, 1980 Revision, Atlantic Richfield Hanford Company, Richland, Washington.

CPS-Z-165-80010, 1999, Rev. D, General Limits, Plutonium Finishing Plant, Criticality Prevention Specification.

CPS-Z-165-80100, 1999, Rev. K, Out of Hood Fissile Material Storage, Plutonium Finishing Plant, Criticality Prevention Specification.

Dobbin, K. D. , 2000, CSER 00-001, Criticality Safety Evaluation Report for Cementation Operations at the PFP, Fluor Federal Service, Richland, Washington.

DOE, 1992, Nuclear Criticality Safety, DOE Order 5480.24, August 12, 1992, U. S. Department of Energy, Washington, D. C.

Erickson, D. G., 1998, MCNP 4B Plutonium Validation, HNF-1905, Rev. 1, CCVR 97-001, Fluor Daniel Northwest, Inc., Richland, Washington.

FDH, 2000, Criticality Safety General, Requirements, HNF-PRO-334, Rev. 1, Fluor Daniel Hanford, Inc., Richland, Washington.

FDH, 1997a, Criticality Safety Control of Fissionable Material, HNF-PRO-537, Rev. 0, Fluor Daniel Hanford, Inc., Richland, Washington.

FDH, 1997b, Criticality Safety Evaluations, HNF-PRO-539, Rev. 0, Fluor Daniel Hanford, Inc., Richland, Washington.

FFS, 1999, Criticality Safety Evaluation Reports, Practice 1342901121.

Greenborg, J. 1999, CSER 98-008: Analysis of Plutonium Compounds as Applied to PFP Criticality Limits for Oxide and Metal Systems, HNF-3572, Rev. 0, Fluor Daniel Hanford, Richland, Washington. 
Lan, J. S., 1999, MCNP Version 4B Approval for Use Documentation \& Authorized User List, FDNW-DSL-99-004, January 12, 1999, Fluor Daniel Northwest, Inc., Richland, Washington.

Marusich, R. M., 1999, CSER 99-003: Criticality Mass of Uranium as Compared to Plutonium - Implications for PFP Processing Uranium, HNF-4436, Rev. 0, Fluor Daniel Hanford, Richland, Washington.

Ramble, A. L., 1999, CSAR 87-004; Plutonium Transfer Wagon PFP CPS-Z-165-80100, H-0 and CPS-Z-165-80110, C- S S-SQA-CSA-064, Rev. 0A, October 19, 1999, B\&W Hanford Company, Richland, Washington.

Schwinkendorf, K. N., 1998, Software Certification Report for MCNP 4B, HNF-3316, Rev. 0, October 14, 1998, Fluor Daniel Northwest, Inc., Richland, Washington.

Watson, W. T., 1997, CSER 96-027, Storage of Cemented Plutonium Residue Container in 55 Gallon Drums, 斯-SD-SQA-CSA-525, Rev. 0, January 13, 1997, Fluor Daniel Northwest, Inc., Richland, Washington. 
HNF-6179 Rev. 0

APPENDIX A - INDEPENDENT REVIEW COMMENTS AND CHECKLIST 
HNF-6179 Rev. 0

This page intentionally left blank 
FLUOR DANIEL NORTHWEST

\section{TECHNICAL PEER REVIEWS}

\section{CHDCKLIST FOR TECHNICAL PEER REVIEW}

Document Reviewed: Title:

\section{Author: \\ Date:}

Scope of Review:
HNF-6179, Rev. 0

CSER 00-006: Storage of Cemented Plutonium Residue Containers in 55 Gallon Drums at the PFP

\section{Yes No* NA}

[4] [ ] [ ]** Previous reviews complete and cover analysis, up to scope of this review, with no gaps.

[4 [ ] [ ] Problem completely defined.

[4 [ ] [ ] Accident scenarios developed in a clear and logical manner.

[4 [ ] [ ] Necessary assumptions explicitly stated and supported.

[ ] [ ] [ 4 Computer codes and data files documentod.

[ ] [ ] [ ]

[ ] [ ] [4

[ ] [ ] [4

[4 [ ] [ ]

[4 [ ] [ ]

Data used in calculations explicitly stated in document.

Data checked for consistency with original source information as applicable.

Mathematical derivations checked including dimensional consistency of results.

Models appropriate and used within range of validity or use outside range of established validity justified.

Hand calculations checked for errors. Spreadsheet results should be treated exactly the same as hand calculations.

[ ] [ ] [ ] Software input correct and consistent with document reviewed.

[ ] [ ] [ ] Software output consistent with input and with results reported in document reviewed.

[4 [ ] [ ] Limits/criteria/guidelines applied to analysis results are appropriate and referenced.

Limits/criteria/guidelines checked against references.

[4 [ ] [ ] Safety margins consistent with good engineering practices.

[4 [ ] [ ] Conclusions consistent with analytical results and applicable limits.

[Y [ ] [ ] Results and conclusions address all points required in the problem statement.

[4 [ ] [ ] Format consistent with applicable guides or other standards.

[] [ ] R Review calculations, comments, and/or notes are attached.

[ 4 [ ] [ ] Document approved (i.e., the reviewer affirms the technical accuracy of the document). 
Independent Peer Reviewer Comments: K. N. Schwinkendorf - 04/20/2000

This CSER was independently peer reviewed by K. N. Schwinkendorf, a qualified Criticality Safety Specialist in the Criticality and Shielding group of Fluor Federal Services. This technical review covered all aspects of this CSER with the exception of checking computer code input and output files. This phase of the review was carried out by S. R. Gedeon, also of the Criticality and Shielding group of Fluor Federal Services. As shown in the two Independent Review Checklists, taken together, this CSER has been reviewed with no gaps.

The construction of base case models using bounding, abnormal but credible conditions was well documented and is conservative. The maximum allowable $\mathrm{k}_{\text {eff }}$ of 0.942 is appropriate for drums of plutonium residue because no large plutonium pieces are anticipated. Although some fine metal phase particles may still be present, they will be well-mixed with other materials such that the system may be considered "non-metal" from a neutronics perspective. For those calculations involving proximately to transport wagons containing metal buttons, this evaluation conservatively uses an allowable $k_{\text {eff }}$ of 0.932 , for a plutonium metal system.

No single contingency violated the criticality safety criterion, and so this reviewer concurs with the conclusion of this CSER, that storage of plutonium residue in 55 gallon drums satisfies double contingency.

Finally, minor editorial comments were suggested, and were incorporated into the final version of the CSER.

Independent Peer Reviewer Comments: S. R. Gedeon - 04/25/2000

Computer inputs, outputs and were checked for consistency and reasonableness. Densities were checked with data in ARH-600 and found equal or conservative.

Input files were checked for model adequacy, material densities, geometry, and container volume. $\mathrm{K}_{\text {eff }}$ values given in the report were verified against output files. Output files were reviewed for adequate convergence. Minor typographical errors were corrected. 


\section{FLUOR DANIEL NORTHWEST}

\section{TECHNICAL PEER REVIEWS}

\section{CHECKLIST FOR TECHNICAL PEER REVIEW}

Document Reviewed: $\quad$ HNF-6179, Rev. 0

Title: $\quad$ CSER 00-006: Storage of Cemented Plutonium Residue Containers

Date: $\quad 4 / 19 / 00$

Scope of Review: Calculations, computer models, "uputs and outputs

\section{Yes No* NA}

[ ] [ ] I/ $]^{* *}$ Previous reviews complete and cover analysis, up to scope of this review, with no gaps.

[ $]$ [ ] [ 1 Problem completely defined.

[ ] [ ] [/, Accident scenarios developed in a clear and logical manner.

[ ] [ ] [/ Necessary assumptions explicitly stated and supported.

[1] [ ] [ ] Computer codes and data files documented.

L] [ ] [ ] Data used in calculations explicitly stated in document.

$X]$ [ ] [ ] Data checked for consistency with original source information as applicable.

V] [ ] [ ] Mathematical derivations chocked including dimensional consistency of results.

L] [ ] [ ] Models appropriate and used within range of validity or use outside range of established validity justified.

LT [ ] [ ] Hand calculations checked for errors. Spreadsheet results should be treated exactly the same as hand calculations.

Y F [ ] [ ] Software input correct and consistent with document reviewed.

L] [ ] [ ] Software output consistent with input and with results reported in document reviewed.

[ ] [ ] [ $\gamma$ Limits/criteria/guidelines applied to analysis results are appropriate and referenced. Limits/criteria/guidelines checked against references. Safety margins consistent with good engineering practices.

Conclusions consistent with analytical results and applicable limits.

Results and conclusions address all points required in the problem statement.

[ ] [ ] $\not Y]$

Format consistent with applicable guides or other standards.

[ ] [ ]** Review calculations, comments, and/or notes are attached.

Y] [ ] [ ] Document approved (i.e., the reviewer affirms the technical accuracy of the document).

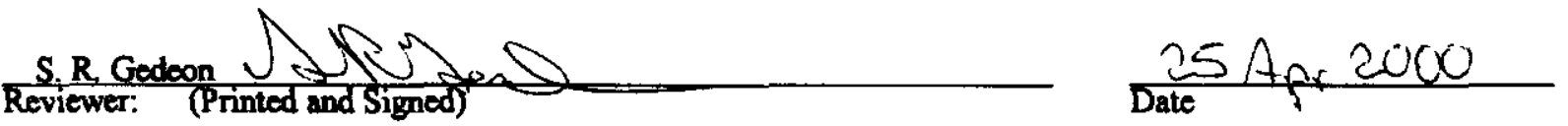

" All "NO" responses muat be explained below or on an additional page.

* Any calculationa, comments, or notes generated as part of thil review should be signed, dated and attuched to this checkliat. Such material should be labeled and recorded in auch a menner an to be intelligible to a technically qualified third party. 
FLUOR DANIEL NORTHWEST

\title{
TECHNICAL PEER REVIEWS
}

\section{CHECKLIST FOR TECHNICAL PEER REVIEW}

\author{
Document Reviewed: HNF-6179, Rev. 0 \\ Title: $\quad$ CSER 00-006: Storage of Cemented Plutonium Residue Containers \\ . in 55 Gallon Drums at the PFP \\ Author: $\quad$ K. D. Dobbin and H. J. Goldberg \\ Date: $\quad 4 / 19 / 00$
}

Scope of Review:

\section{Yes No* NA}

[ ] [ ] $X]^{* *}$ Previous reviews complete and cover analysis, up to scope of this review, with no gaps.

[x] [ ] [ ] Problem completely defined.

[D [ ] [ ] Accident scenarios developed in a clear and logical manner.

[X] [ ] [ ] Necessary assumptions explicitly stated and supported.

[P] [ ] [ ] Computer codes and data files documented.

[X] [ ] [ ] Data used in calculations explicitly stated in document.

[ ] [ ] [ Data checked for consistency with original source information as applicable.

[ ] [ ] [ [9] Mathematical derivations checked including dimensional consistency of results.

[y] [ ] [ ] Models appropriate and used within range of validity or use outside range of established validity justified.

[ ] [ ] [ ] Hand calculations checked for errors. Spreadsheet results should be treated exactly the same as hand calculations.

[ $x$ [ [ ] [ ] Software input correct and consistent with document reviewed.

[ ] [ ] I Software output consistent with input and with results reported in document reviewed.

[ [ [ ] [ ] Limits/criteria/guidelines applied to analysis results are appropriate and referenced.

(X) Limits/criteria/guidelines checked against references.

[ $X]$ [ ] [ ] Safety margins consistent with good engincering practices.

[x] [ ] [ ] Conclusions consistent with analytical results and applicable limits.

$[x]$ [ ] [ ] Results and conclusions address all points required in the problem statement.

[ ] [ ] I I Format consistent with applicable guides or other standards.

[X] [ ]** Review calculations, comments, and/or notes are attached.

[ [ ] [ ] Document approved (i.e., the reviewer affirms the technical accuracy of the document).

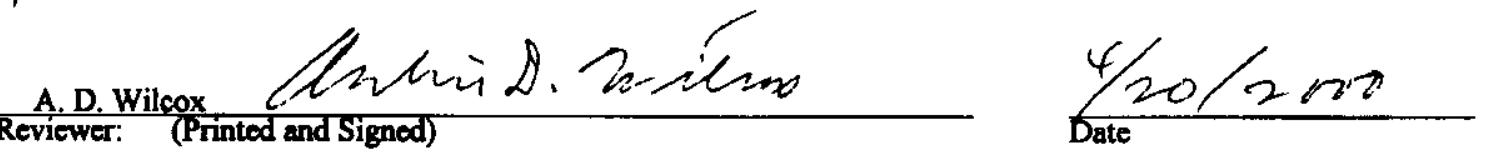

* All "NO" responses must be explained below or on an additional page.

- Any caleulations, comments, or notee generated as part of this review should be signed, dated and attached to this checkliat. Such material should be labeled and recorded in such a manner as to be intelligible to a technically qualified third party. 


\section{Mohr and Associates \\ ENGINEERING-ANALYSIS-CONSULTING}

1440 AGNES STREET

RICHLAND. WA 99352

509-946-0943

FAX 509-946-4395

April 20, 2000

To: K. D. Dobbin, Criticality and Shielding, Fluor Federal Services, Inc.

From $\triangle$. D. Wilcox, Mohr and Associates

Subj: Review of CSER 00-006 "STORAGE OF CEMENTED PLUTONIUM RESIDUE CONTAINERS IN 55 GALLON DRUMS AT THE PFP"

CSER 00-006 was reviewed. The CSER addresses the transport to storage and the storage of 55-gallon drums containing cemented plutonium residue from the PFP. The analyses considered a range of contingencies involving interactions within a drum: interactions between drums; and between a drum or drums and the wagon used to transport plutonium cans. The results of these analyses show that the drums may be safely transported and stored in the PFP. The administrative control of the total amount of plutonium (or equivalent fissile material) allowed in each drum, 200 grams of $\mathrm{Pu}$, is the most important restriction on the whole process.

The input listings that are part of the subject CSER were reviewed and found adequate. The output listings were not reviewed.

CSER 96-027 and CSER 00-001 were used to provide background information pertinent to CSER 00-006. These CSERs describe the cementation of the plutonium bearing waste and the storage of the cemented containers in 55 gallon drums.

Some editorial comments were transmitted orally to you and have been incorporated into the CSER.

After reviewing CSER 00-006, I find that the analysis covers the range of contingencies adequately and demonstrates that the 55 gallon drums may be safely transported and stored in the PFP.

Sincerely,

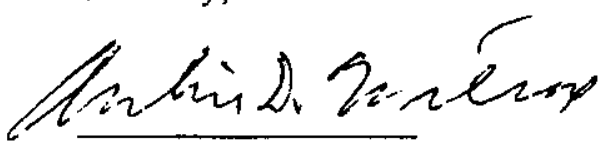

Archie D. Wilcox 
HNF-6179 Rev. 0

This page intentionally left blank.

A-8 
HNF-6179 Rev. 0

APPENDIX B - MCNP COMPUTER CODE VALIDATION

B-1 
HNF-6179 Rev. 0

This page intentionally left blank 


\section{B.1 VALIDATION PROCEDURE}

Validation of the computer code methods in this analysis consisted of testing the code and neutron cross sections on calculations of known critical configurations. These benchmark experiments have fissile isotopes in systems similar to that evaluated by this CSER. The computed and measured $\mathrm{k}_{\mathrm{eff}} \mathrm{s}$ for the benchmark configurations were compared to establish a bias that includes the uncertainty in the calculational methods. A bias-adjusted $\mathrm{k}_{\text {eff }}$ for the benchmark systems was defined to include both the deviations of the calculated from the measured $\mathrm{k}_{\mathrm{eff}} \mathrm{s}$, and experimental and calculational uncertainties along with the spread in the ability of the computer code to calculate similar systems. In addition, criticality safety criteria require that the bias-adjusted $\mathrm{k}_{\mathrm{eff}}$ for CSER analysis calculations not exceed the established $\mathrm{k}_{\mathrm{eff}}$ safety limit at the $95 \%$ confidence level.

This method is illustrated in Figure B.1. Critical is defined as a $\mathrm{k}_{\mathrm{eff}}$ of unity, adjusted by the bias established from the comparison of calculations with benchmarks. The bias is combined with the safety margin of 0.05 (a safety limit that $k_{\text {eff }}$ must be less than or equal to 0.95 ) to compare with the calculated value and statistical uncertainty of the computer calculated $\mathrm{k}_{\mathrm{eff}}$ 's of this CSER analysis. The calculated target $\mathrm{k}_{\text {eff }}$ is established by adding the bias, 0.05 , and 1.645 times the one-sigma uncertainty of the calculated $\mathrm{k}_{\mathrm{eff}}$ for the particular CSER analysis and subtracting that value from 1.0. For the analyses in this CSER, all the computer statistical uncertainties were less than \pm 0.002 , so this value was used to set the target $\mathrm{k}_{\mathrm{eff}}$ 's as described in Section B.2.

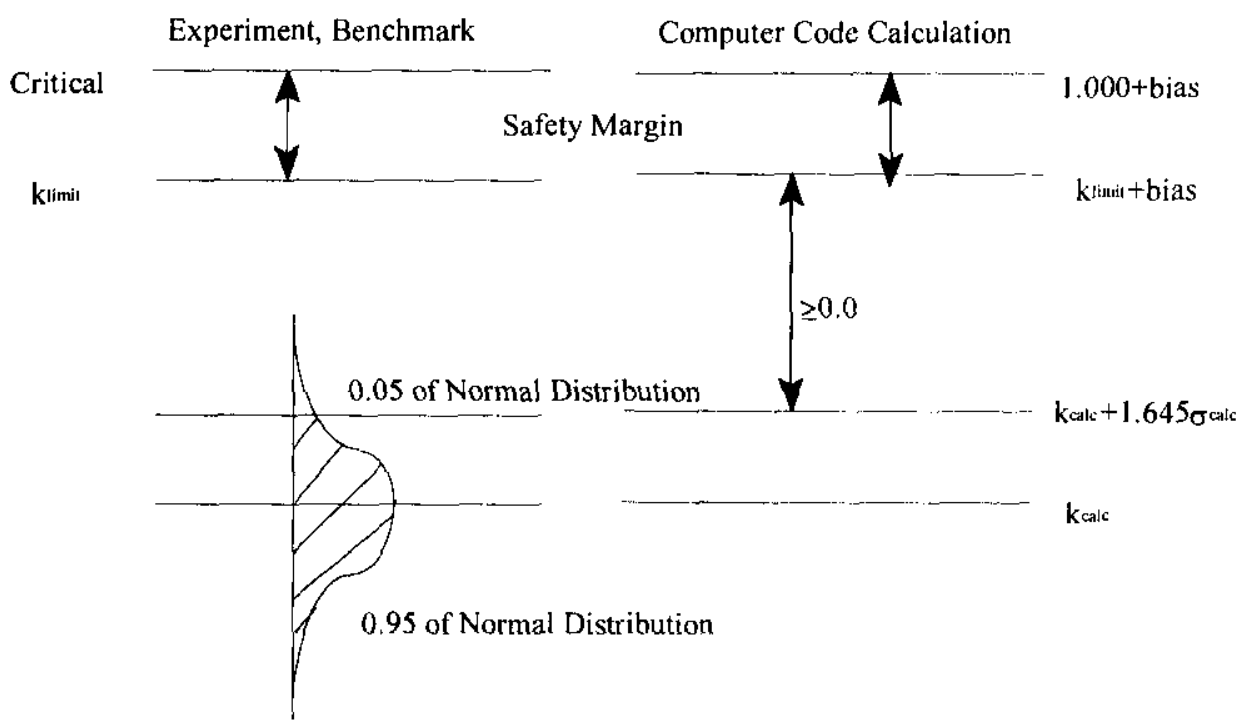

Figure B.1. Logic of Validation Procedure 
HNF-6179 Rev. 0

\section{B.2 GENERIC VALIDATION FOR PLUTONIUM SYSTEMS}

A report by J. S. Lan, MCNP Version 4B Approval For Use Documentation \& Authorized User List (Lan 1999), presents the results of calculations to determine a generic bias for plutonium configurations, as encountered in the Plutonium Finishing Plant. One hundred and forty three benchmark experiments were calculated. There were different material types that were considered in the plutonium validation calculations:

- Plutonium metal,

- Plutonium oxide,

- Plutonium solutions,

- Plutonium solutions with cadmium (a neutron poison),

- Water and polystyrene moderators, and

- Water, plexiglass, paraffin, polyethylene, and steel and concrete reflectors

The lower tolerance limit $b_{L}$ was calculated for the benchmark experiments such that there is $95 \%$ confidence that $95 \%$ of the benchmark calculated $\mathrm{k}_{\text {eff }}$ 's is above that limit. This is expressed by the following formula:

$$
\mathrm{b}_{\mathrm{L}}=\mathrm{k}_{\mathrm{avg}}-\mathrm{K}_{\mathrm{b}} * \sigma_{\mathrm{avg}}
$$

where: $b_{L} \quad=$ lower tolerance limit for $95 \%$ confidence that $95 \%$ of the benchmark calculated $\mathrm{k}_{\text {eff }}$ 's is above this limit,

$\mathrm{k}_{\mathrm{avg}}=$ the average of the $\mathrm{k}_{\text {eff }}$ 's calculated by MCNP 4B,

$\mathrm{K}_{\mathrm{b}} \quad=$ a multiplier found from statistical tables for non-central $\mathrm{t}$-distribution, and depends on number of degrees of freedom, and

$\sigma_{\mathrm{avg}} \quad=$ standard deviation of the MCNP k $\mathrm{eff}_{\mathrm{e}} \mathrm{s}$.

Bias is calculated by the following formula:

$$
\text { bias }=\mathrm{b}_{\mathrm{L}}-\mathrm{k}_{\mathrm{crit}}
$$

where:

$$
\begin{aligned}
\mathrm{k}_{\mathrm{crit}}= & \text { the average of the } \mathrm{k}_{\mathrm{eff}} \mathrm{s} \text { for the critical experiments; for the plutonium experiments } \\
& \mathrm{k}_{\mathrm{crit}}=1.000 .
\end{aligned}
$$

The bias for the plutonium metal group was significantly different than for all other groups. For this reason, it was concluded that separate bias values for metal and non-metal groupings would be appropriate. The lower tolerance limit for the metal group (17 benchmark critical experiments) calculated to be 0.9884 . The lower tolerance limit for the non-metal group (126 benchmark critical experiments) calculated to be 0.9991 . These lower tolerance limits yielded the bias appropriate for each material category:

- $\quad$ Plutonium metal

- Plutonium non-metal bias is -0.0116 , bias is -0.0009 . 
For conservatism, these calculated biases were recommended to be increased to:

- Plutonium metal

- Plutonium non-metal

recommended bias is -0.0150 , recommended bias is -0.0050 .

The safety criteria for future calculations on undetermined systems requires that the bias-adjusted $\mathrm{k}_{\text {eff }}$ does not exceed 0.95 at the $95 \%$ confidence level. This is expressed by the following formula:

$$
\mathrm{k}_{\text {eff }}=\mathrm{k}_{\text {calc }}-\text { bias }+1.645 * \sigma_{\text {caic }} \leq \mathrm{k}_{\text {limit }}
$$

where: $\mathrm{k}_{\text {calc }} \quad=\mathrm{k}$ value given by MCNP 4B calculation for system in question,

bias $=-0.015$ for Pu metal, and -0.005 for Pu non-metal systems,

$1.645=$ a constant number of standard deviations for .95 of the distribution for a one-sided standard normal distribution

$\sigma_{\text {calc }}=$ standard deviation given by MCNP 4B calculation for system in question, and

$\mathrm{k}_{\text {limit }} \quad=0.95$ for plutonium systems, generally.

$\mathrm{k}_{\text {limit }}$ is generally taken to be 0.95 for plutonium systems.

For a standard deviation $\left(\sigma_{\text {calc }}\right)$ of 0.002 or less, the $k_{\text {eff }}$ value for plutonium metal systems is:

$$
\begin{gathered}
\mathrm{k}_{\text {calc }}-(-0.015)+1.645 * 0.002 \leq 0.95 \text {, or } \\
\mathrm{k}_{\text {calc }} \leq 0.95+(-0.015)-1.645 * 0.002=0.932
\end{gathered}
$$

On this basis, it is determined that the true $\mathrm{k}_{\text {eff }}$ of an analyzed configuration with plutonium will not exceed 0.95 with a $95 \%$ confidence level for plutonium metal systems if the calculated value $\left(\mathrm{k}_{\text {calc }}\right.$, and $\sigma \leq 0.002$ ) is limited to a maximum value of $\mathbf{0 . 9 3 2}$.

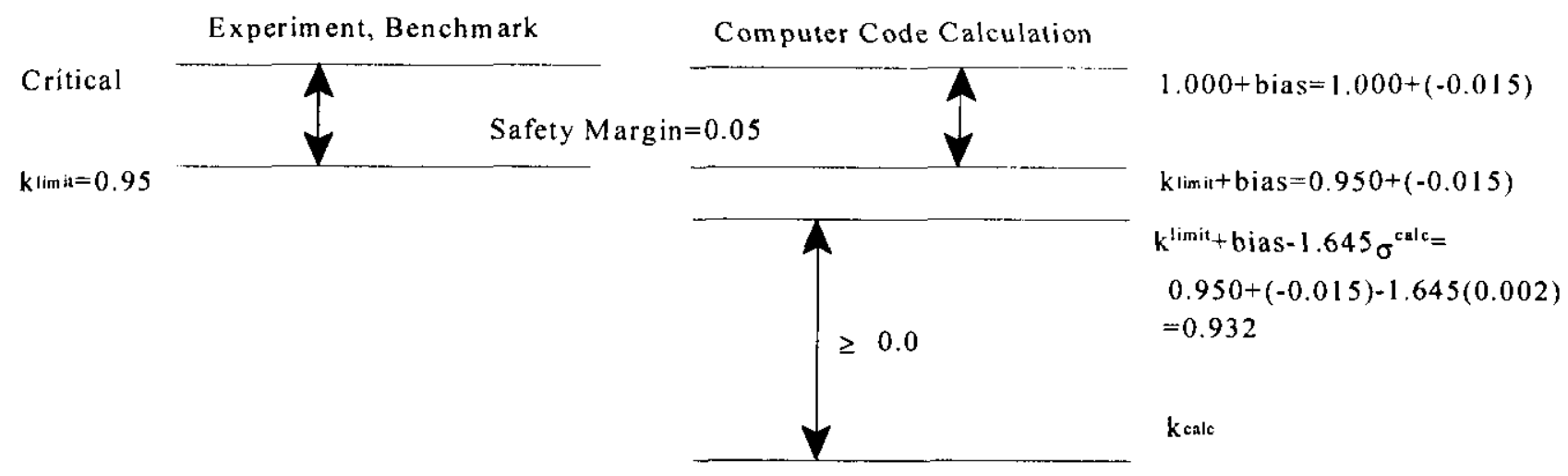

Figure B.2. Implementation of Validation Procedure 
For a standard deviation $\left(\sigma_{\text {calc }}\right)$ of 0.002 or less, the $\mathrm{k}_{\text {eff }}$ value for non-metal systems is:

$$
\begin{gathered}
\mathrm{k}_{\text {calc }}-(-0.005)+1.645 * 0.002 \leq 0.95, \text { or } \\
\mathrm{k}_{\text {calc }} \leq 0.95+(-0.005)-1.645 * 0.002=0.942
\end{gathered}
$$

On this basis, it is determined that the true $\mathrm{k}_{\text {eff }}$ of an analyzed configuration with plutonium will not exceed 0.95 with a $95 \%$ confidence level for plutonium non-metal systems if the calculated value $\left(k_{\text {calc }}\right.$, and $\sigma \leq 0.002$ ) is limited to a maximum value of $\mathbf{0 . 9 4 2}$.

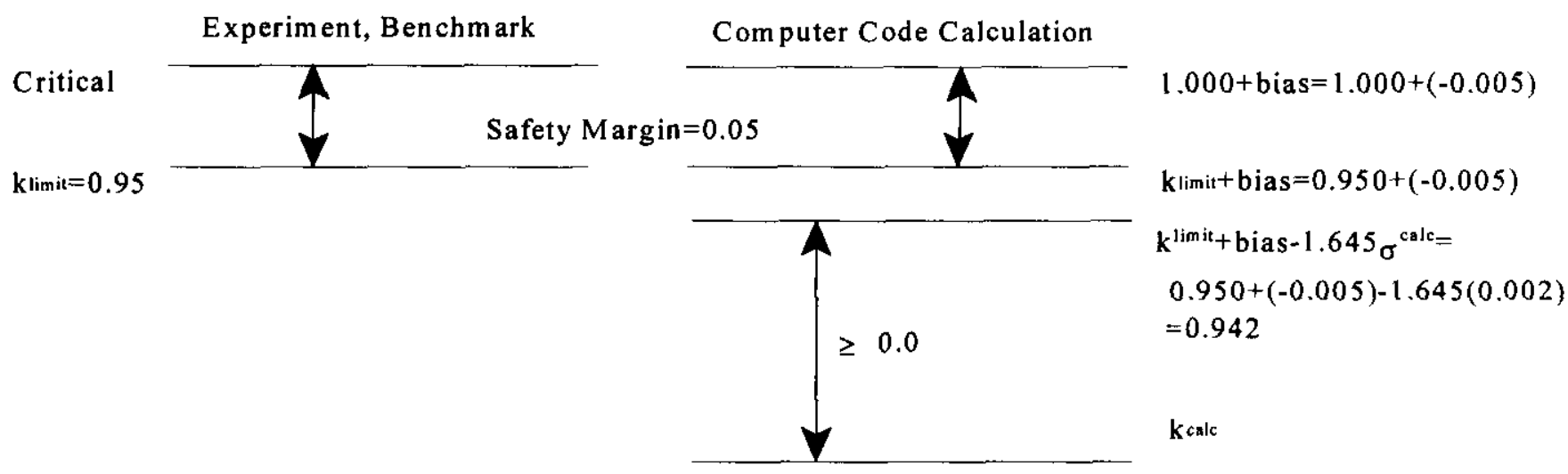

Figure B.3 Implementation of Validation Procedure

\section{B.3 VALIDATION OF MCNP 4B}

The validation of the MCNP4B code on the new computing system, Intergraph ${ }^{\mathrm{TM}}, 400 / 450$ MHz Pentium II, personal computers was documented in Lan, 1999. The essence of the validation was cross-correlation of calculational results obtained with this code version and results of critical experiments, as reported in MCNP Version $4 B$ Approval for Use Documentation \& Authorized User List (Lan 1999).

TM Intergraph is a trademark of the Intergraph Corporation of Huntsville, Alabama. Pentium is a trademark of Intel Corporation, Santa Clara, California. 
HNF-6179 Rev. 0

APPENDIX C - MCNP INPUT FILES

C-1 
HNF-6179 Rev. 0

This page intentionally left blank.

C-2 
HNF-6179 Rev. 0

\section{dblbtch_botlap}

Ihree Billets in a Non-pipe 55 Gallon Drum

c. three overpacked Pu billets at the bottom of a $400 \mathrm{~g} / \mathrm{drum}$ Filled with poly

silicon replaces Calcuum

Cell Defintions

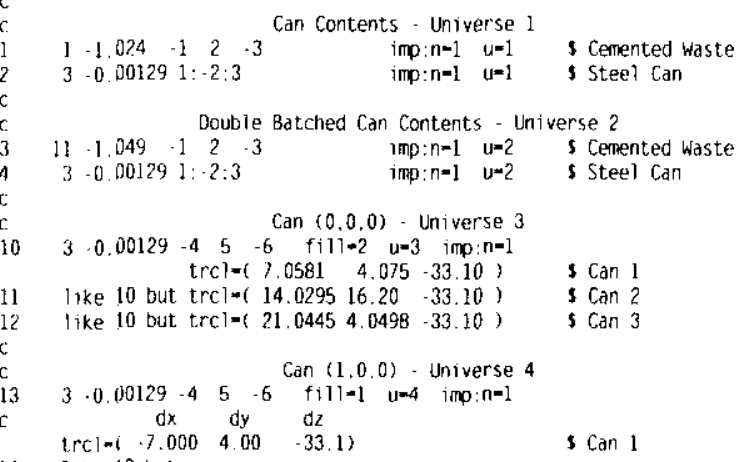

S Radius of Waste

3 Botton of Waste 3 Top of Waste $\checkmark$ Outside of Can

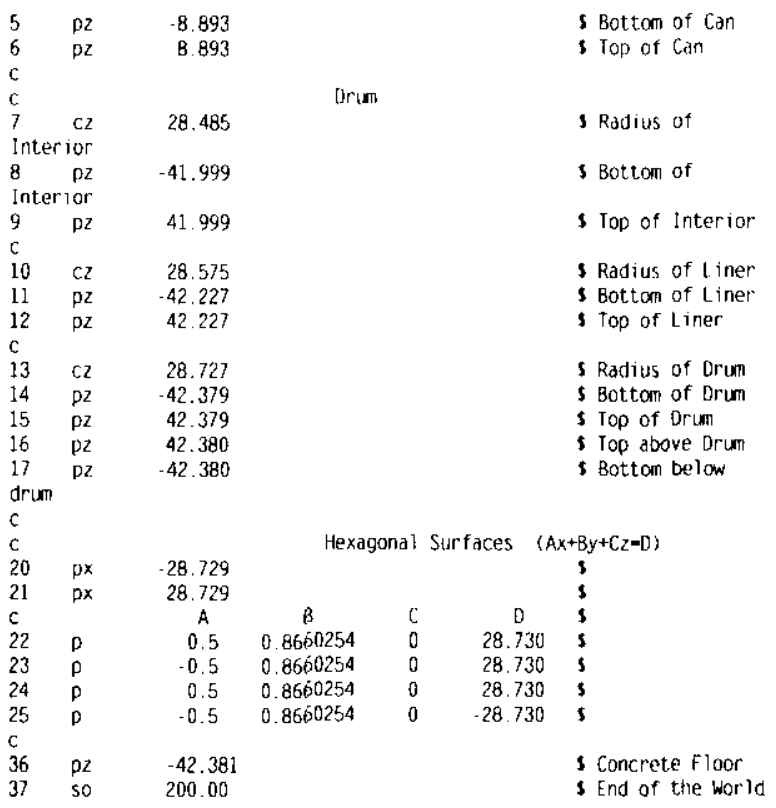

\section{mode $\mathrm{n}$}

kcode $3000 \quad 1.0 \quad 10 \quad 100$

ksrc 15. $20 . \quad 33$.

40. $20 .-33$

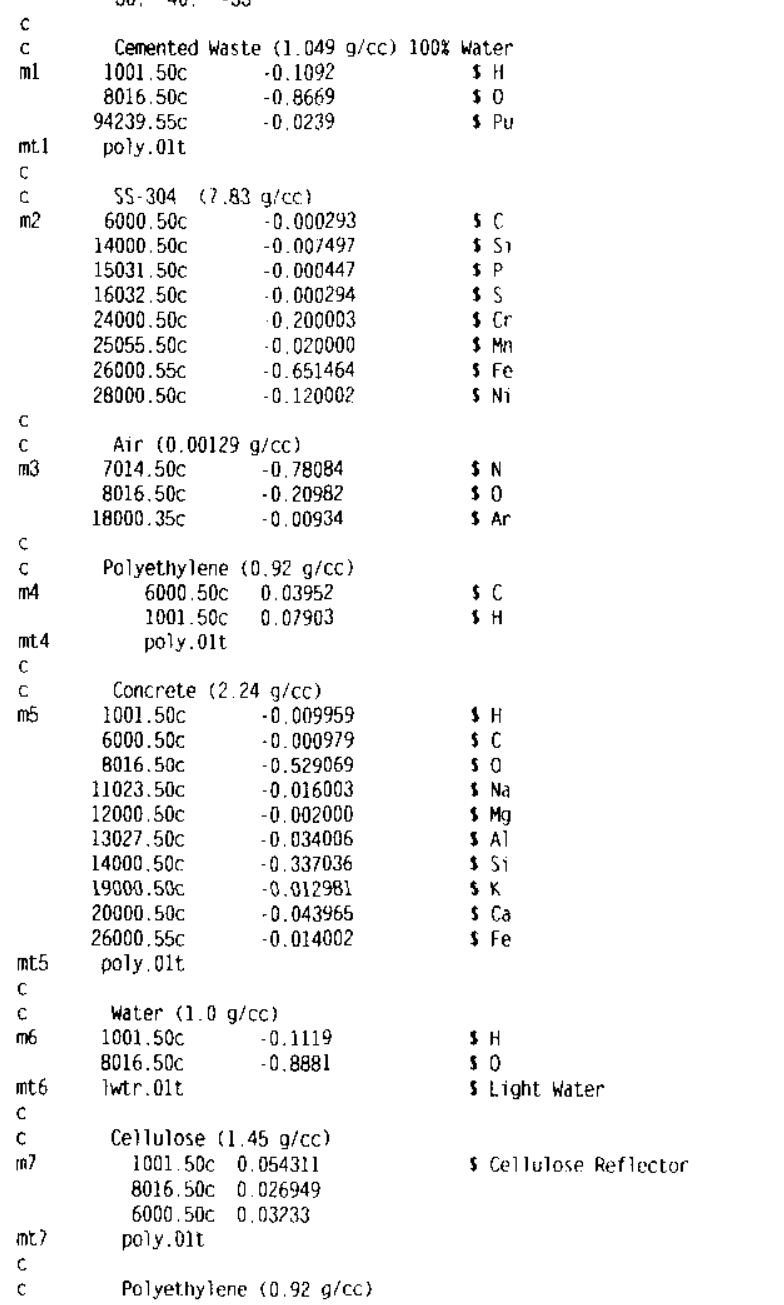


HNF-6179 Rev. 0

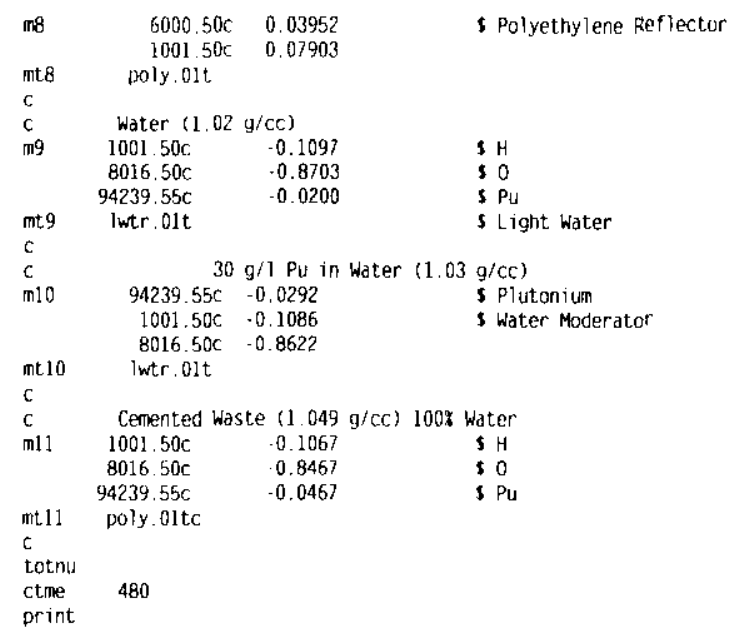

ablotch_bot1p

Three Billets in a Non-pipe 55 Gallon Drum

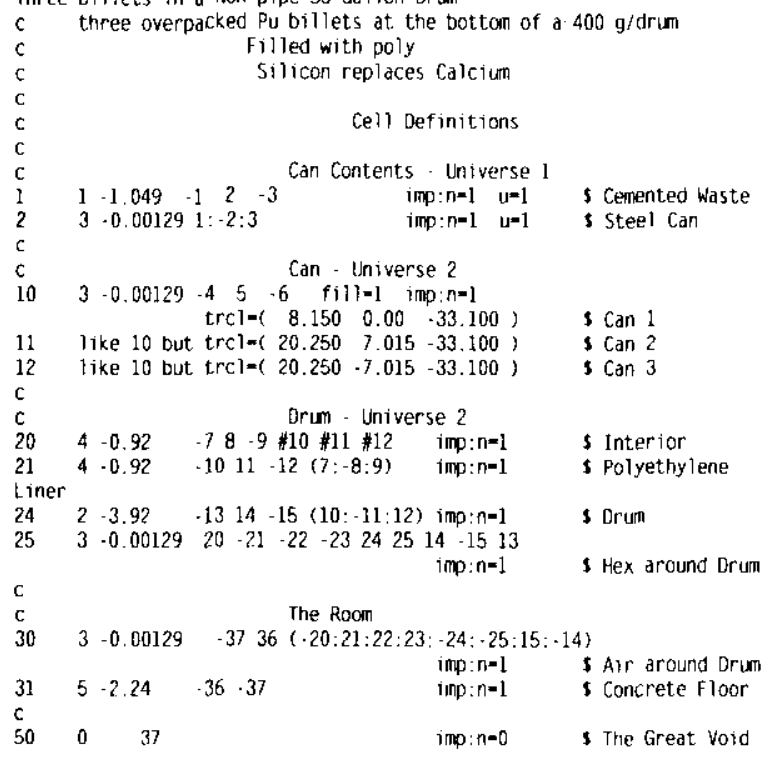

c

-8.890
8.890

6.988

$-8.893$

B. 893

28.485

.41 .999

41.999

28.575

42.227

42.227

28.727

$-42.379$

42.379

42.380

Surface Definitions

Billets

5 Radius of Waste Botton of Waste 5 Top of Waste

s Outside of Can

s Bottom of Can

- Top of can

Drum

s Radius of

s Botton of

5 Top of Interior

5 Radius of Liner

Bottom of Liner

s Top of Liner

$\$$ Radius of Drum

$s$ Bottom of Drum

5 Top of Drum

s Top above Drum

Hexagona! Surfaces $(A x+B y+C z=D)$

s

$\begin{array}{llccccc}\mathrm{C} & & \mathrm{A} & \mathrm{B} & \mathrm{C} & \mathrm{D} & \text { s } \\ 22 & \mathrm{p} & 0.5 & 0.8660254 & 0 & 28.730 & \text { s } \\ 23 & \mathrm{p} & -0.5 & 0.8660254 & 0 & 28.730 & \text { s } \\ 24 & \mathrm{p} & 0.5 & 0.8660254 & 0 & -28.730 & \text { s } \\ 25 & \mathrm{p} & -0.5 & 0.8660254 & 0 & -28.730 & \mathbf{s} \\ \mathrm{C} & & & & & & \text { s Concrete Floor } \\ 36 & \mathrm{pZ} & -42.381 & & & & \text { s End of the world } \\ 37 & \text { so } & 100.00 & & & \end{array}$

$\begin{array}{lllll}\text { mode } n & & & & \\ \text { kcode } & 3000 & 1.0 & 10 & 100\end{array}$

$\begin{array}{llll}\text { ksrc } & 8.150 & 0.00 & -33.100\end{array}$

C Cemented Waste $(1.049 \mathrm{~g} / \mathrm{cc}) 100 \%$ Water

C Cemented Waste $(1.049 \mathrm{~g} / \mathrm{cc})$ 100\% Water $\begin{array}{rrr}8016.50 \mathrm{C} & -0.8467 & 50 \\ 94239.55 \mathrm{C} & -0.0467 & 5 \mathrm{PL}\end{array}$

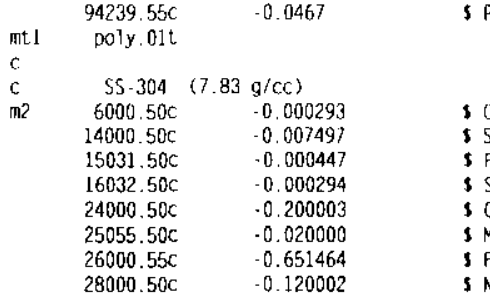

$\mathrm{C} \quad$ Air $(0.00129 \mathrm{~g} / \mathrm{CC})$

$\begin{array}{rrr}7014.50 \mathrm{C} & -0.78084 & \text { S N } \\ 8016.50 \mathrm{C} & -0.20982 & 50 \\ 18000.35 \mathrm{C} & -0.00934 & \text { A }\end{array}$

c Polyethylene $(0.92 \mathrm{~g} / \mathrm{cc})$

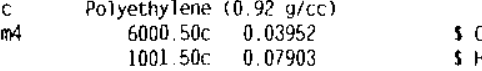

mt4 poly.01t

c Concrete $(2.24 \mathrm{~g} / \mathrm{cc})$

m5 $1001.50 c \quad-0.009959$

$6000.50 \mathrm{C} \quad 0.000979$ is

$\begin{array}{rrr}8016.50 \mathrm{C} & -0.529069 & \text { s } 0\end{array}$

$\begin{array}{rrr}11023.50 \mathrm{C} & 0.016003 & \text { SNa } \\ 12000.50 \mathrm{C} & -0.002000 & \end{array}$

$12000.50 \mathrm{C}-0.002000 \quad$ S MG

$13027.50 \mathrm{C} \quad-0.034006$ S A

$14000.50 \mathrm{C}-0.337036 \quad$ s Si

$19000.50 \mathrm{C}-0.012981$ s $\mathrm{K}$

$26000.55 \mathrm{C}-0.014002 \quad$ s Fe

c Water $(1.0 \mathrm{~g} / \mathrm{cc})$

m6 $\begin{array}{lll}1001.50 \mathrm{C} & -0.1119 & \text { SH } \\ 8016.50 \mathrm{C} & -0.8881 & \text { S } \mathrm{O}\end{array}$

mt6 Iwtr.01t S Light Water

c Cellulose $(1.45 \mathrm{~g} / \mathrm{cc})$

$1001.50 \mathrm{c} \quad 0.054311$

$8016.50 \mathrm{C} \quad 0.026949$

$6000.50 \mathrm{C} \quad 0.03233$

mt 7 poly.01t

c Polyethylene $(0.92 \mathrm{~g} / \mathrm{cc}$

$\begin{array}{llll}\mathrm{C} & 6000.50 \mathrm{c} & 0.03952 & \text { S Polyethylene Reflector }\end{array}$

$\begin{array}{lll}1001.50 \mathrm{C} & 0.07903\end{array}$

mt8 poly.01t

c $\quad$ Water $(1.049 \mathrm{~g} / \mathrm{cc})$

$\mathrm{mg} \quad 1001.50 \mathrm{C} \quad-0.1067$

$8016.50 \mathrm{C}-0.8467$ \$ $\mathrm{H}$

$94239.55 \mathrm{C}-0.0467$

mt9 Iwtr.01t s ight Woter

c

totnu

ctme 480

print

dbibtch_ref2p

Three Billets in a Non-pipe 55 Gallon Drum

three Pu billets at the bottom of d $400 \mathrm{~g} / \mathrm{drum}$ one relecting boundary (two drums). Poly

Cell Definitions

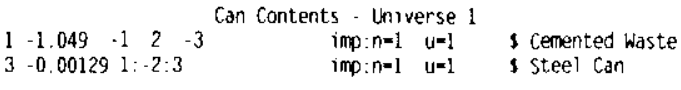


HNF-6179 Rev. 0

10 $3-0.00129-4 \quad 5 \quad 6 \quad$ Can - Universe 2

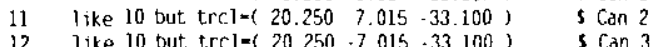

$\begin{array}{ll}\mathrm{C} & \text { Drum - Universe 2 }\end{array}$

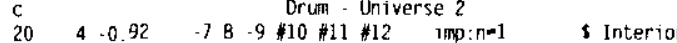

$214-0.92 \quad-1011-12(7:-8: 9)$ imp:n=1 s Polyethylene

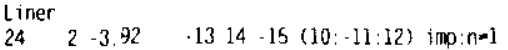

$\begin{array}{llllllllllll}25 & 3 & -0.00129 & 20 & \cdot 21 & -22 & -23 & 24 & 25 & 14 & \cdot 15 & 13\end{array}$

c The Room

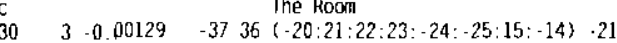

$\begin{array}{lllllll}31 & 5-2.24 & -36-37-21 & \text { imp:n-1 } & \text { s Air around Orun } \\ & & \text { Concrete Floor }\end{array}$

5 $10 \quad 37: 21$

imp: $n=0 \quad 3$ The Great void

5
6
$c$
$c$

c

$7 \quad \mathrm{C} 2$

8 p2

Interior

c p

$10 \quad c z \quad 28.575$

$\begin{array}{rrr}11 & p z & -42.227 \\ 12 & p z & 42.227\end{array}$

Surface Definitions

Billets

$\begin{array}{lrl}c z & 6.985 & \\ p z & -8.890 & \\ p z & 8.890 & \\ c z & 6.988 & \\ 02 & -8.893 & \\ p z & 8.893 & \\ & & \text { Drum }\end{array}$

$\begin{array}{lll}3 \mathrm{CZ} & 28.727\end{array}$

$14 \quad \mathrm{pz} \quad-42.379$

$\begin{array}{lll}15 & \text { pz } & 42.379 \\ 16 & \text { pz } & 42.380\end{array}$

16
$c$

$\mathrm{c}$

$\begin{array}{llr}20 & p x & -28.729 \\ \star 21 & p x & 28.729 \\ \end{array}$

$\mathrm{C}$
22
23
24
25
$\mathrm{c}$
36

23
24
25
$\mathrm{c}$
36
37

0.5

$\begin{array}{cc}A & B \\ 0.5 & 0.8660254 \\ -0.5 & 0.8660254 \\ 0.5 & 0.8660254\end{array}$

Hexagonal Surfaces $(A x+B y+C z=D)$

$\begin{array}{rr}0.5 & 0.8660254 \\ -0.5 & 0.8660254\end{array}$

0
28.730
28.730
-28.730
-28.730

-42.381
100.00

$-28.730$

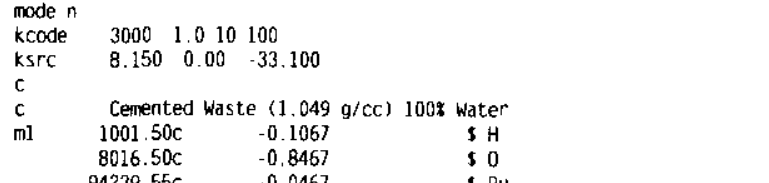

5 Radius of Waste 5 Botton of Waste S Top of Waste 5 Outside of can

$\checkmark$ Botton of Can

$\checkmark$ Top of Can

3 Radius of

s Bottom of

$\$$ Top of interior

5 Radius of Liner 3 Bottom of Liner 3 Top of Liner

5 Radius of Drum

$\$$ Bottom of Drum

5 Top of Drum

Top above Drum

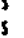

s Concrete floor 5 End of the World

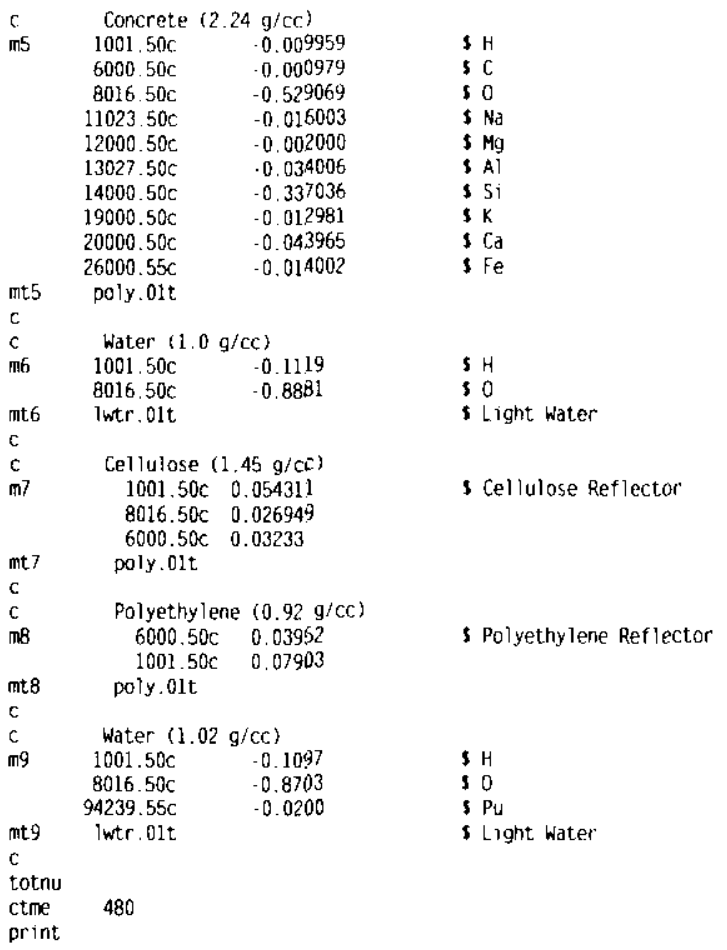

dolbtch_refp

Three Billet,s in a Non-pipe 55 Gallon Drum

C three Pu billets at the bottom of a $400 \mathrm{~g} / \mathrm{drum}$ Infinite reflection-Poly

Cell Definitions

$$
\begin{array}{llllll}
1 & -1.049-1 & 2 & -3 \quad \text { Can Contents Universe } 1 \\
\text { inp: } n=1 & u=1
\end{array} \text { \& Cenented Waste }
$$

Can - Universe 2

$\begin{array}{llllllll}3-0.00129 & -4 & 5 & -6 & \text { fill }=1 \text { imp: } n=1\end{array}$ trel $=\left(\begin{array}{lll}8.150 & 0.00 & -33.100\end{array}\right)$

ike 10 but $\operatorname{trc}=(20.250-7.015-33.100)$ s Can 2

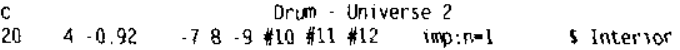

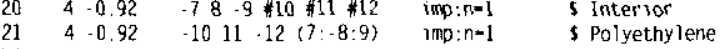

$\begin{array}{llllllll}24 & 2 & -3.92 & -13 & 14 & -15 & (10-11: 12) \text { ime:n=1 } & \text { s Drum }\end{array}$

$25 \quad 3 \quad-0.00129 \quad 20-21 \quad-22 \begin{array}{lllllllll}25 & 24 & 25 & 14 & -15 & 13\end{array}$

$\begin{array}{llllllllllll} & 31 & 5 & -2.24 & 38 & -14 & 20 & -21 & -22 & 23 & 24 & 25\end{array}$

$\begin{array}{llllllllll}3 & -0.00129 & -37 & 15 & 20 & -21 & -22 & -23 & \text { imp: } n=1\end{array}$

1 Hex around Drum

s Concrete floor

- Air Above Drums

$50 \quad 0 \quad(-20: 21: 22: 23:-24:-25: 37:-38) \quad 1$ 1mp: $: n=0$

s The Great void

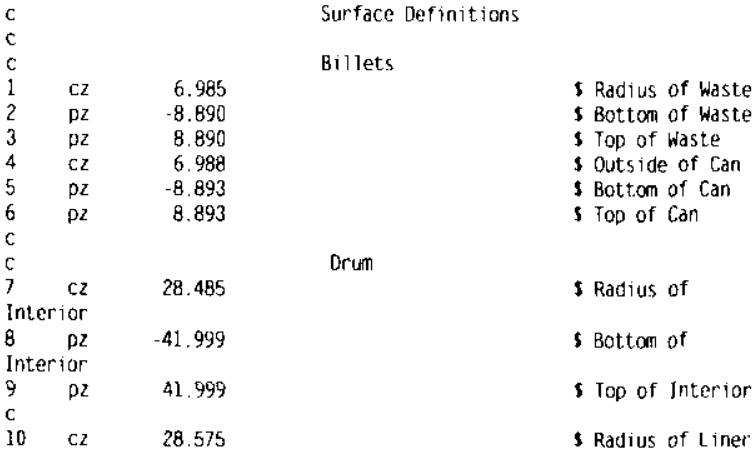


HNF-6179 Rev. 0

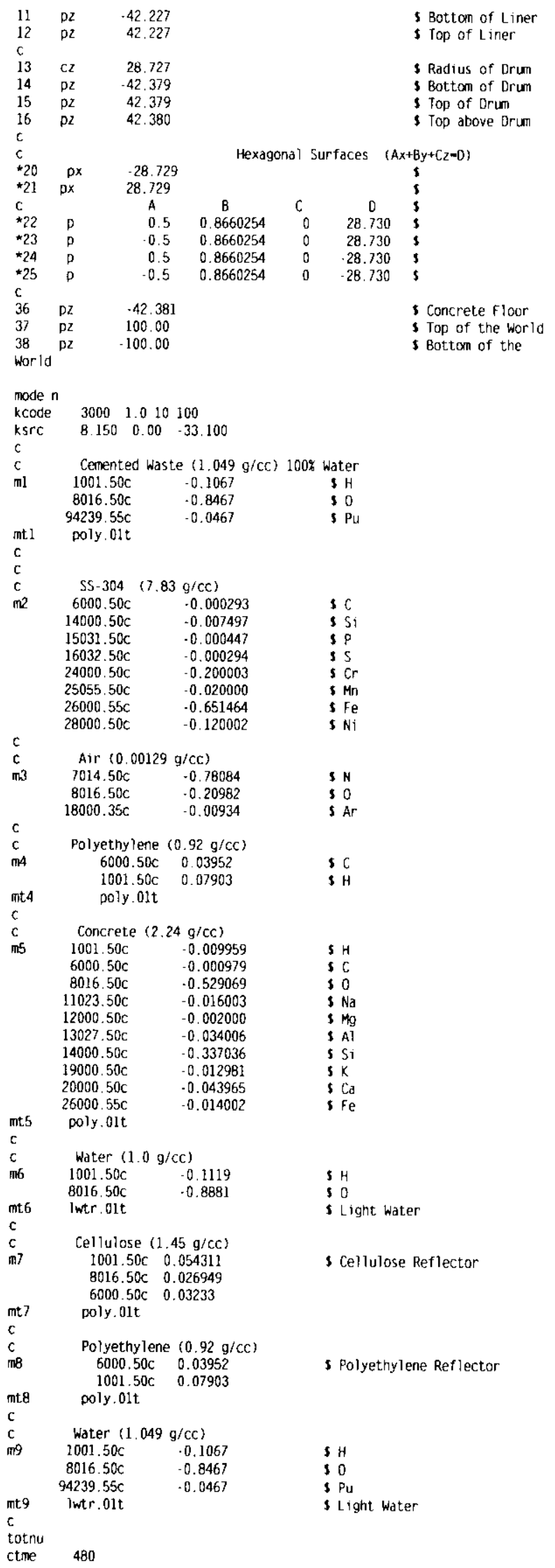

print

ablotch_refrotp

Three Billets in a Non-pipe 55 Gallon Drum $c$ three Pu billets at the bottom of a $400 \mathrm{~g} / \mathrm{drum}$ Infinite reflection-poly

Cell Definitions

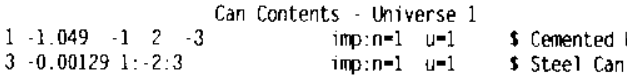

$\begin{array}{lll}16 & 02 & 42.380 \\ c & & \end{array}$

\$ Top of Interior

5 Radius of Liner s Bottom of Liner 5 Top of Liner.

s Radius of Drum s Botton of Drum s Top of Drum

5 Top above Drum

$\begin{array}{lll}20 & p \times & .28 .729\end{array}$

*21 px 28.729

$\mathrm{C}$

*22

$\begin{array}{ll} \\ * 25 & p\end{array}$

$\begin{array}{lll}\mathrm{C} & & \\ 36 & \mathrm{pz} & -42.38\end{array}$

world s Bottom of the

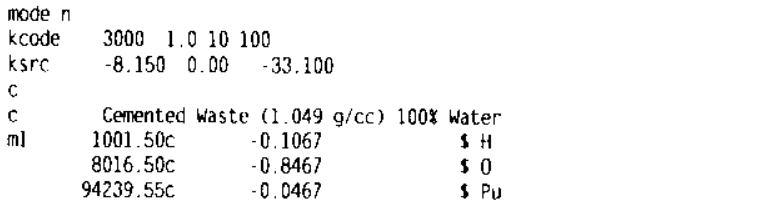


HNF-6179 Rev. 0

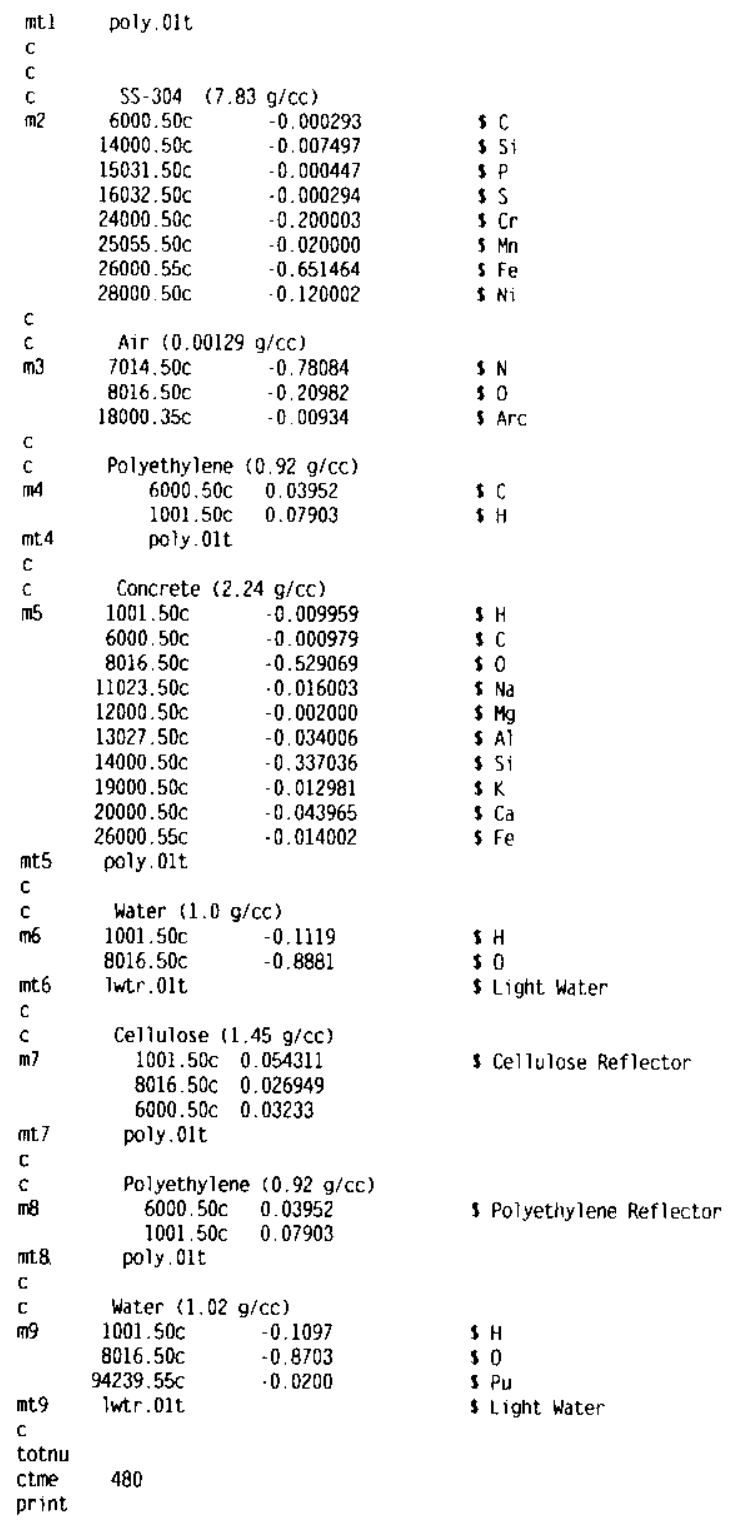

nonpipe_botlp.inp

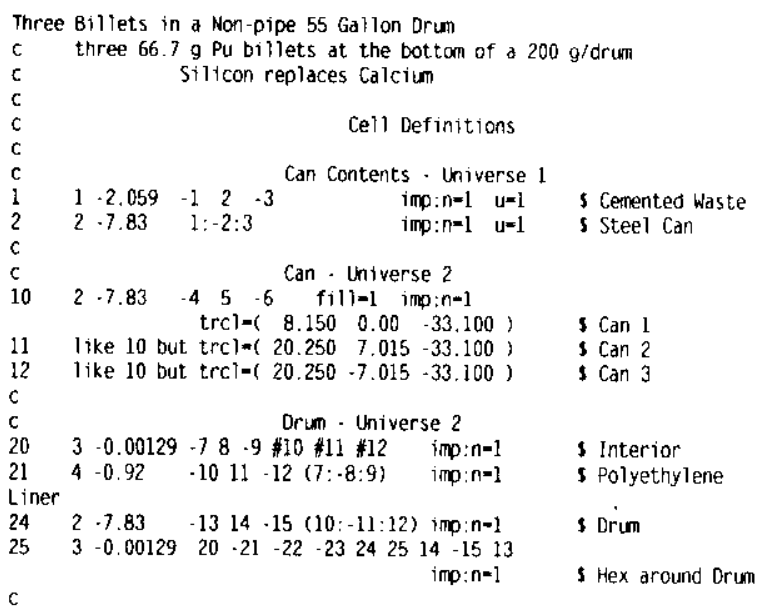

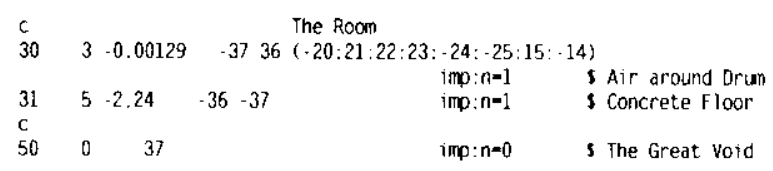

\begin{tabular}{|c|c|c|c|c|c|c|}
\hline$c$ & & & Surf & De & nitions & \\
\hline$c$ & & & Bill & & & \\
\hline 1 & $c z$ & 6.985 & & & & s Radius of Waste \\
\hline 2 & $p z$ & -8.890 & & & & s Bottom of Waste \\
\hline 3 & $p z$ & 8.890 & & & & S Top of Waste \\
\hline 4 & $c 2$ & 6.988 & & & & S Outside of Can \\
\hline 5 & pz & .8 .893 & & & & s Botton of can \\
\hline 6 & p2 & 8.893 & & & & 3 Top of Can \\
\hline $\begin{array}{l}c \\
c\end{array}$ & & & Drut & & & \\
\hline 7 & $\mathrm{CZ}$ & 28.485 & & & & S Radrus of \\
\hline Inter & ior & & & & & \\
\hline $\begin{array}{l}8 \\
\text { Inter }\end{array}$ & $\begin{array}{l}p Z \\
\text { ior }\end{array}$ & -41.999 & & & & \$ Bottom of \\
\hline 9 & $\mathrm{pz}$ & 41.999 & & & & s Top of Interior \\
\hline c & & & & & & \\
\hline 10 & $C 2$ & 28.575 & & & & S Radius of Liner \\
\hline Il & $\mathrm{pz}$ & $-42,227$ & & & & s Bottom of Liner \\
\hline 12 & $p z$ & 42.227 & & & & s Top of Liner \\
\hline$c$ & & & & & & \\
\hline 13 & $c z$ & 28.727 & & & & s Radius of Drum \\
\hline 14 & $p z$ & -42.379 & & & & s Bottom of Drum \\
\hline 15 & $p Z$ & 42.379 & & & & 3 Top of Drum \\
\hline 16 & $\mathrm{p} 2$ & 42.380 & & & & \$ Top above Drum \\
\hline c & & & & & & \\
\hline$c$ & & & Hexas & |al & faces & $(x+B y+\{z=D)$ \\
\hline 20 & $p x$ & .28 .729 & & & & $s$ \\
\hline 21 & $p x$ & 28.729 & & & & 3 \\
\hline c & & A & B & $\mathrm{C}$ & D & 3 \\
\hline 22 & $\rho$ & 0.5 & 0.8660254 & 0 & 28.730 & 3 \\
\hline 23 & p & -0.5 & 0.8660254 & 0 & 28.730 & $s$ \\
\hline 24 & $p$ & 0.5 & 0.8660254 & 0 & -2.8 .730 & 5 \\
\hline 25 & $p$ & -0.5 & 0.8660254 & 0 & -28.730 & 5 \\
\hline $\mathrm{C}$ & & & & & & \\
\hline 36 & p2 & .42 .381 & & & & Concrete Floor \\
\hline 37 & 50 & 100.00 & & & & 5 End of the world \\
\hline
\end{tabular}

mode $n$

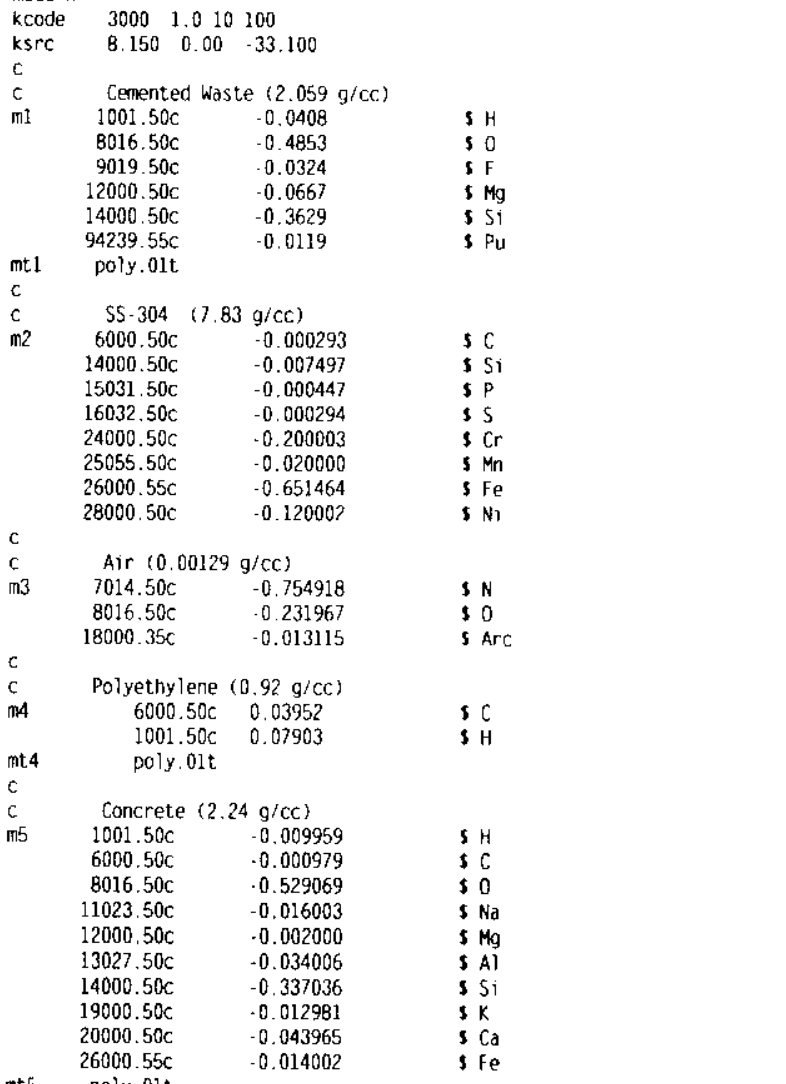


HNF-6179 Rev. 0

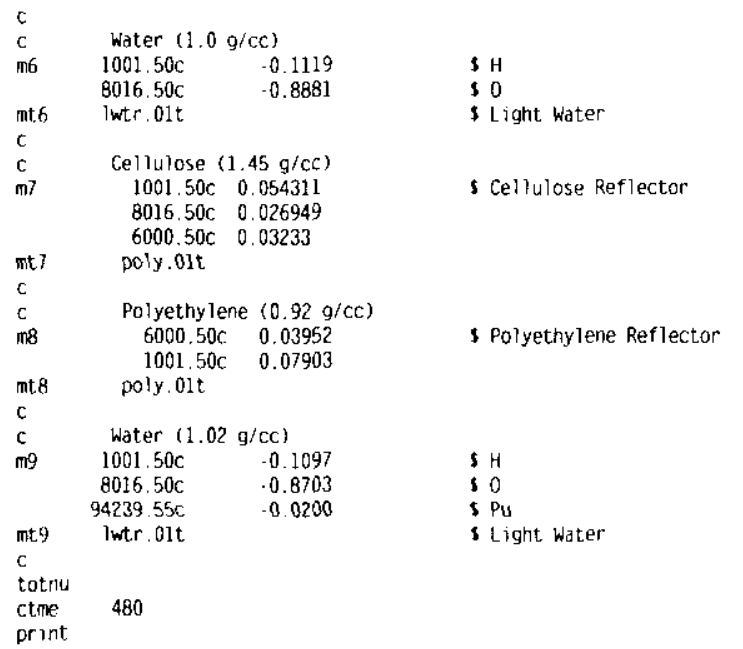

nonpipe bot2p. inp

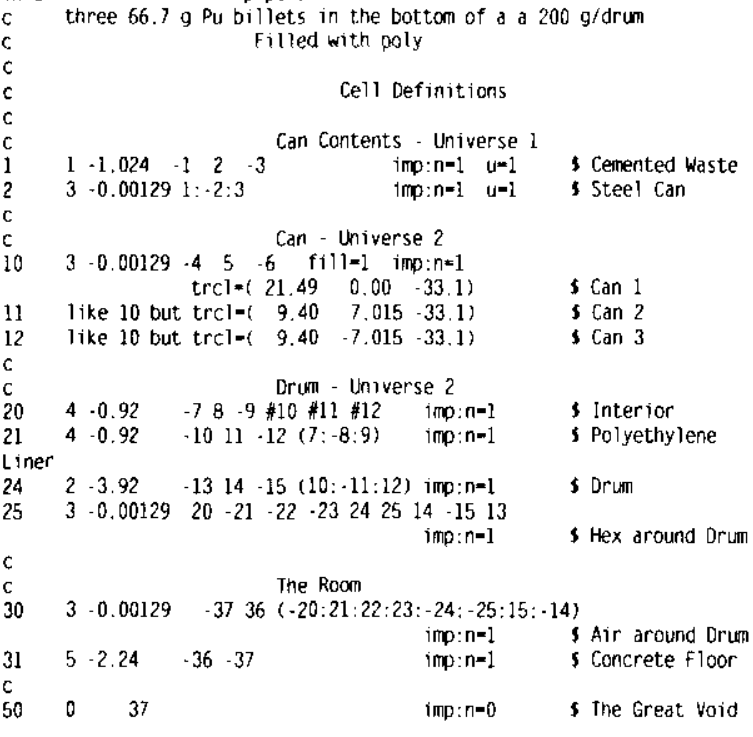

$\mathrm{C}$
$\mathrm{c}$
$\mathrm{c}$
1
2
3
4
5
6
$\mathrm{c}$

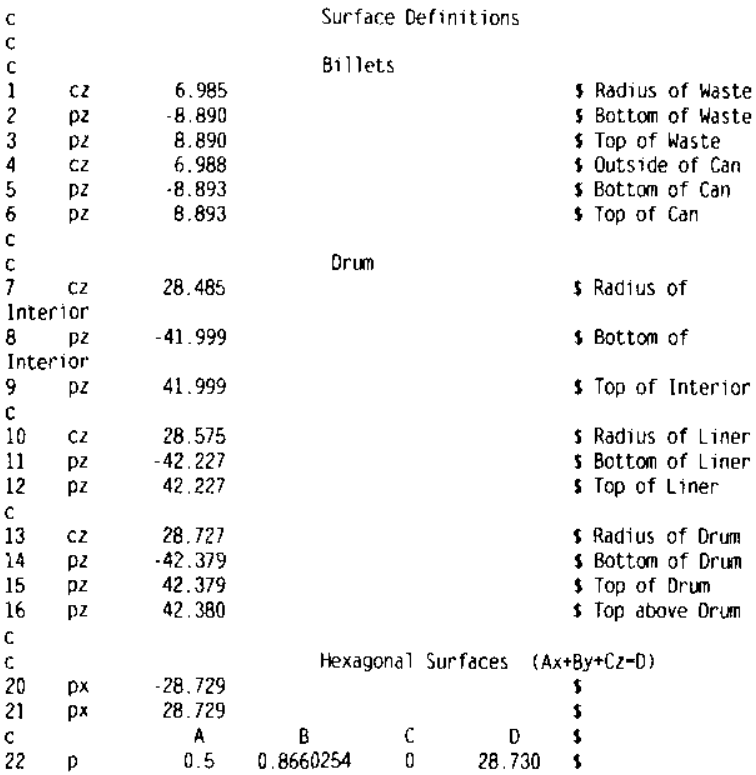

$\begin{array}{llrrrrl}23 & p & -0.5 & 0.8660254 & 0 & 28.730 & \text { s } \\ 24 & p & 0.5 & 0.8660254 & 0 & -28.730 & \text { s } \\ 25 & 0 & -0.5 & 0.8660254 & 0 & -28.730 & \text { s } \\ c & & & & & & \text { s Concrete Floor } \\ 36 & p 2 & -42.381 & & & & \text { s End of the world } \\ 37 & \text { so } & 100.00 & & & \end{array}$

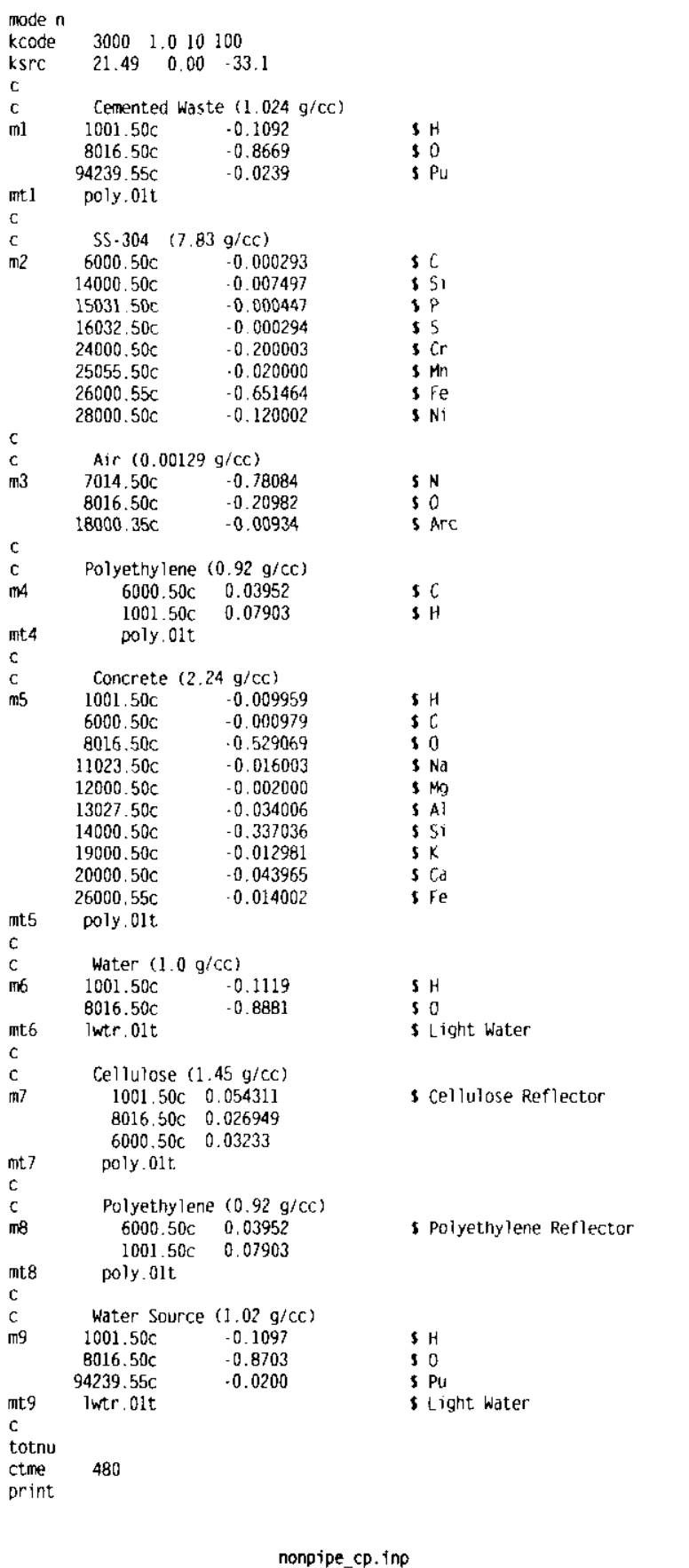

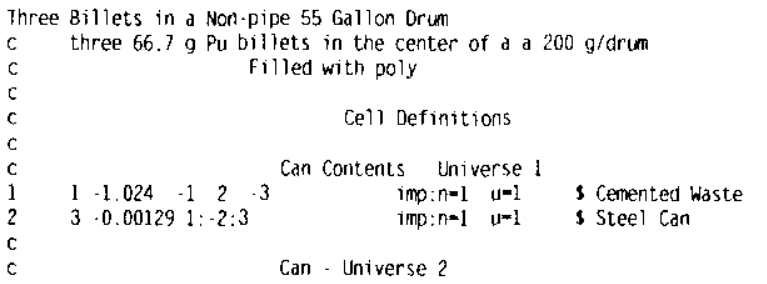


HNF-6179 Rev. 0
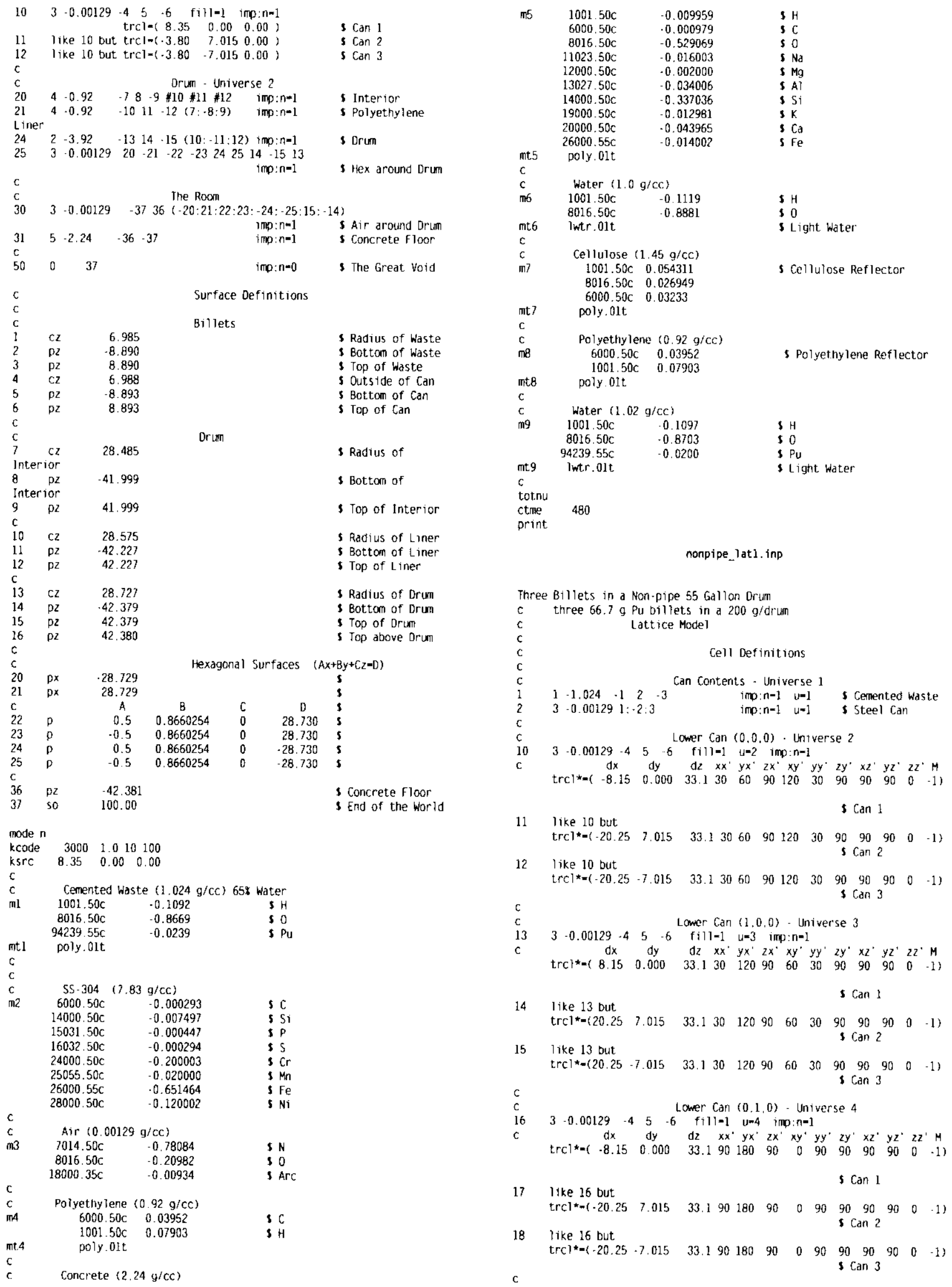


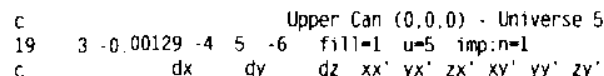

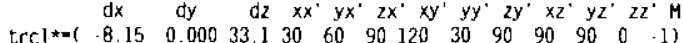

$20 \quad$ like 19 but

5 Can 1

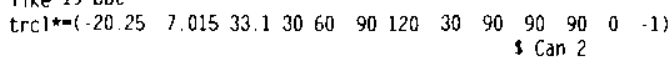

21 like 19 but

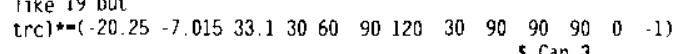

Upper Can $(1,0,0)$. Universe 6

$22 \quad 3-0.00129-4 \quad 5 \quad-6 \quad$ fill $=1 \quad u=6 \quad$ imo: $n=1$

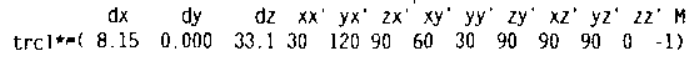

$23.11 \mathrm{ke} 22$ but

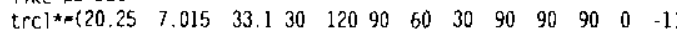

$24 \quad$ like 22 but

$5 \operatorname{can} 2$

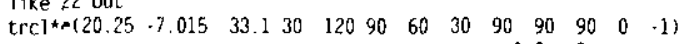
5 Can 3

$\begin{array}{llllllll}\text { C } & \text { Upper Can }(0,1,0) \text {. Universe ? } \\ 25 & 3 & -0.00129 & -4 & 5 & -6 & \text { fill-1 } u=7 \text { imp: } n-1\end{array}$

c $d x \quad d y \quad d z \quad x x^{*} y x^{\prime} 2 x^{\prime} x y^{\prime} y y^{\prime} z y^{\prime} x z^{\prime} y z^{\prime} z z^{\prime} M$

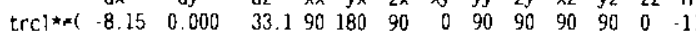

26 like 25 put

s Can 1

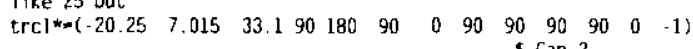

27 like 25 but

s Can 2

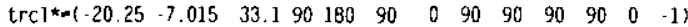
5 Can 3

c

c Drum - Universe 2

$30 \quad 4 \quad-0.92 \quad-78 \quad-9 * 10 * 11 \quad \# 12 \quad$ imp: $n=1 \quad u=2$ s Interior

$31 \quad 4 \cdot 0.92 \quad-1011 \cdot 12(7: \cdot 8: 9)$ imp:n-1 u=2 s Polyethylene

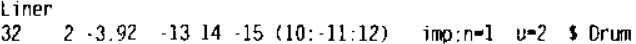

$33 \quad 3 \cdot 0.0012913:-14: 15$ imp:n-1 $u=2$ \& Air around Drum

c Drum - iniverse 3

$34 \quad 4-0.92 \quad .78 .9 \# 13 \quad \# 14 \quad \# 15 \quad$ imp: $n=1 \quad u=3$ s interior

$\begin{array}{llllll}35 & 4 & -0.92 \quad-1011 \cdot 12(7:-8: 9) \quad \text { imp:n=1 } u=3 & \text { s Polyethylene }\end{array}$

Liner

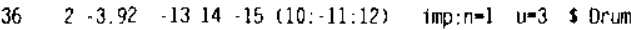

$37 \quad 3 \cdot 0.0012913:-14: 15 \quad$ imp:n-1 $y=3$ s Air around Drum

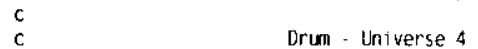

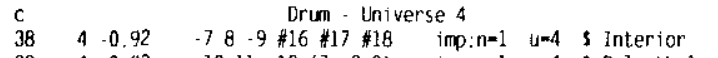

$39 \quad 4-0.92 \quad \cdot 1011-12(7:-8: 9) \quad$ imp:n=1 $\quad u=4$ s Polyethylene

Liner

$40 \quad 2-3.92 \quad-1314 \cdot 15(10:-11: 12) \quad$ imp:n-1 u=4 S Drum

$413 \cdot 0.0012913:-14: 15$ imp:n=1 $u=4$ \& Air around orumc

c $\quad$ Drum - Universe 5

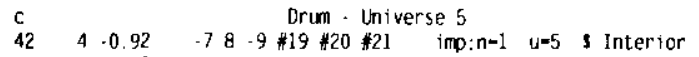

$\begin{array}{lllll}4 & 4 & -0.92 & -1011-12(7:-8: 9) \quad \text { imp: } n=1 \quad u=5 & \text { S Polyethylene }\end{array}$

$\begin{array}{llllllll}44 & 2 & -3.92 & .13 & 14 & -15 & (10: \cdot 11: 12) \quad \text { imp:n-1 } u=5 & \text { s Orum }\end{array}$

$45 \quad 3-0.0012913:-14: 15$ imp:n=1 $v=5$ s Air around Drum

C $\quad$ Drum - Universe 6

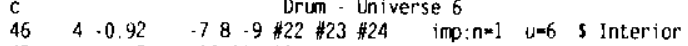

$\begin{array}{llllll}47 & 4 & -0.92 & -10 & 11-12(7:-8: 9) \quad \text { imp:n=1 } u=6 & \text { s Polyethylene }\end{array}$

Liner

$\begin{array}{lllllllll}48 & 2 & -3.92 & -13 & 14 & -15 & (10:-11: 12) \quad i m p: n-1 \quad u=6 & s & \text { Drum }\end{array}$

$493-0.00129$ 13:-14:15 imp:n=1 u=6 Air around orum

C Drum - Universe 7

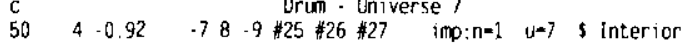

$51 \quad 4-0.92 \quad-1011-12(7:-8: 9) \quad$ imp:n=1 $u=7$ s Polyethylene

Liner

$52 \quad 2 \cdot 3.92-1314-15(10: \cdot 11: 12) \quad$ imp:n-1 u-7 s Orum

$53 \quad 3 \quad-0.00129$ 13:-14:15 imp:n=1 u=7 3 Air around Drumc

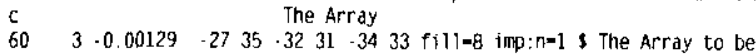
filled c

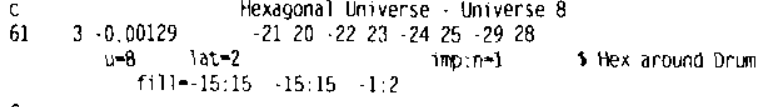

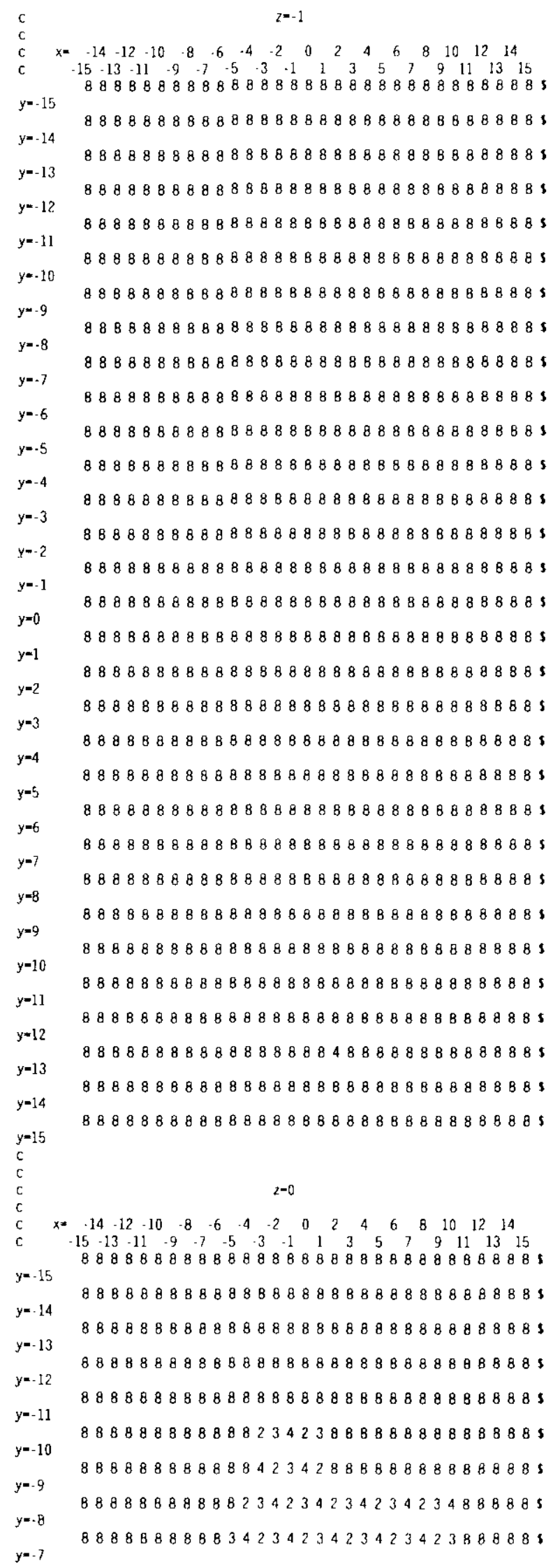


HNF-6179 Rev. 0

$y=-6$

$y=-5$

$y=-4$

$y=-3$

$y=-2$

$y=-1$

$y=0$

$y=1$

$y=2$

$y=3$

$y=4$

$y=5$

$y=6$

$y=7$

$y \star 8$

$y=9$

$y=10$

$y=11$

$y=12$

$y=13$

$y=14$

$y=15$

c

C $y=-15$

$y=-14$

$y=-13$

$y=-12$

$y=-11$

$y=-10$

$y=-9$

$y=-8$

$y=-7$

$y=-6$

$y=-5$

$y=-4$

$y=-3$

$y=-2$

$y=1$

$y=0$

$y=1$

$y=2$

$y=3$

$y-4$
$868888888823423423423423428888 B$ s $8888888888423423423423423488888 \mathrm{~s}$ 8888888888342342342342342388888 s 8888888884234234234234234288888s $888868888342342342342342348888 B$ s $88888888823423423423428888888 B 8$ s 888888888423423423423488 B8888885 88888834234234234234238888888885 B8 888882342342342342342888888888s 88888842342342342342388888888885 88888834234234234234288888888885 88688823423423423423488888868885 88888842342342342342388888888885 $8888883423423423423428888 B 888 B 85$ 888888234234234234234888888888 B 8888884234234232886888888888888 s 88888834234234238888888888888983 88888823423423428888888888888885 8888888864234234889888888888 88 88888888834234238888888888888885 88888868823423428888888888888885 88888888842342348888888888888885

$z=1$

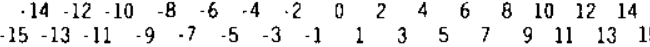
88888 B 8888888888B88888888888888s 88 8 8888 B B8888888888888888888888 $8888888 B 88888888888888888888888$ s $888888888888888888 B 888888888888$ s

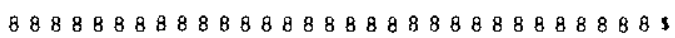
88888888888888888888888888886885 $8 B 88888 B 88888888888888888888888$ s 8888888888888888888888888888888 s 8 88888888888888888888888 B888888, $8 B 8888888888888888 B 8888888888885$

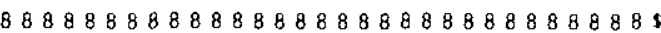

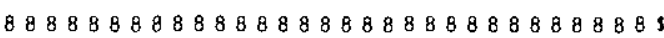
88888888888888888888888888888885 8888888888888888888888888888888 s 8888888888888888888888888888888 s 8888888888888888888888888888888 s 8888888888888888888888888888888 s 8888888688888888888888888888888

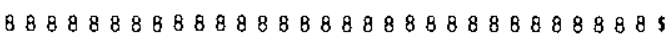
8888888688888888888888888888888 s $y=5$

$y=6$

$y=7$

$y=8$

$y=9$

$y=10$

$y=11$

$y=12$

$y=13$

$y=14$

$y=15$

$c$$$
\text { c }
$$

$y=-15$

$y=-14$

$y=-13$

$y=-1$ ?

$y=-11$

$y=-10$

$y=.9$

$y=-8$

$y=-7$

$y=-6$

$y=-5$

$y=-4$

$y=-3$

$y=-2$

$y=1$

$y=0$

$y=1$

$y-2$

$y=3$

$y=4$

$y=5$

$y=6$

$y=7$

$y=8$

$y=9$

$y=10$

$y+11$

$y=12$

$y=13$

$y=14$

$y=15$
$C$

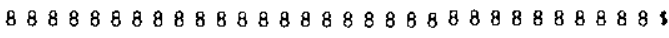
$888888888 B 888888888888888888888$ s 8868888888888688888888888888888 s $8 B 8888888888 B 888888888888$ 8 88888 s B888888888R8R888888888886888888

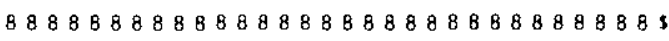

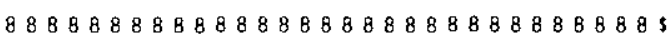
8888888888888888888888888886888 s $8 B 88868888 B 88888848 B 88888888888$ s

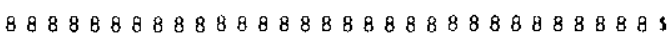
$888688888 B 8888 B 888888$ B 88888B8885

$z=2$

$\begin{array}{lllllllllllllll}-14 & -12 & -10 & -8 & -6 & -4 & -2 & 0 & 2 & 4 & 6 & 8 & 10 & 12 & 14\end{array}$

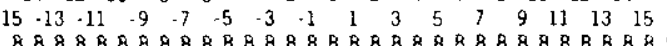
8888888888888888888888888888888 s $88 B 8888888888 B 8888 B 8688888888885$ 8888888888888888888888888888888 s B $888888888888888 B 88888888888 B 885$ 88888888888888888888888888888885 8 $8888888 B 8888888888886888888888$ เ 8888888888888888888888888888888 ,

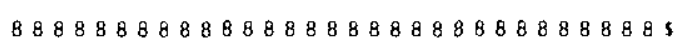
B8888888888888888888868888888885 $888888888888 B 888888888888888888 \mathrm{~s}$

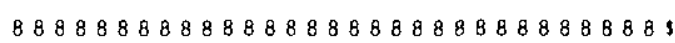
$8888 B 8888 B 8888 B 88888 B 8888 B 88888$ s

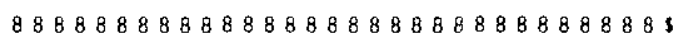
$8888888 B 88888888888888888888888$ s $888888888 B 88888 B 888888888888888$ s $888 B 888888888888888868888888888$ s $888888888888888888888888888888 B$ s 8888888888888888888888888888888 s

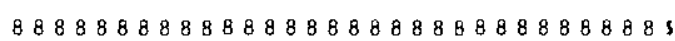

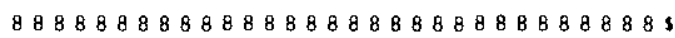
8888888888888888886888888888888 s 8888888888888888888886888888888 s $888888888 B 88888 B 8 B 8888 B 88888888$ s 8888886888888888888888888888888 s $88888 B 888888888888888888888888 \mathrm{~B}$ 8 888888888888688888888888888888 s $868888 B 888888888888888888888888$ s B $88888 B 8888888888488 B 8888888888$ s $8888888888 B 88888888888888888888$ s $88 B 88888 B 8 B 888 B 88888 B 8888888888$, 
HNF-6179 Rev. 0$$
\text { c }
$$

$\begin{array}{lllllllll}70 & 3 & -0.00129 & -32 & 31 & -34 & 33 & -36 & 27 \\ \text { imp }: n=1\end{array}$

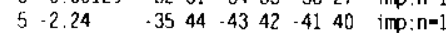

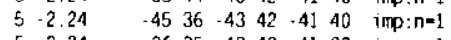

$\begin{array}{lllllllll}5 & -2.24 & .36 & 35 & -43 & 42 & -41 & 32 & \text { imp: } n=1\end{array}$

$\begin{array}{lllllllll}5 & -2.24 & -36 & 35 & -43 & 42 & -31 & 40 & \text { imp }: n=1\end{array}$

11

$\begin{array}{lllllllllll}75 & 5 & \cdot 2.24 & -36 & 35 & -33 & 42 & -32 & 31 & \mathrm{~m} m \mathrm{~m}: \mathrm{n}-1\end{array}$

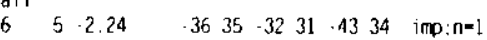
wall

$\begin{array}{lll}\text { C } & 0 & 41:-40: 43:-42: 45:-44 \quad \text { imp: } n=0\end{array}$

$\mathrm{c}$
$\mathrm{c}$
$\mathrm{c}$
1
2
3
4
5
6
$\mathrm{c}$
$\mathrm{c}$

\section{C2 $\quad 6.985$}

$02-8.890$

pz $\quad 8.890$

$C 2 \quad 6.988$

$\begin{array}{lr}\text { pz } & -8.893 \\ \text { pz } & \text { 8.893 }\end{array}$

C2 28.485

$\begin{array}{lll}\text { Interior } & \\ 8 & \text { pz } & -41.999\end{array}$

$\begin{array}{lll}\text { Interior } & \\ 9 & \text { pz } & 41.999\end{array}$

$\begin{array}{lll}\mathrm{C} & & \\ 10 & \mathrm{CZ} & 28.575 \\ 11 & \mathrm{pz} & -42.227\end{array}$

$\begin{array}{lll}11 & \mathrm{pz} & -42.227\end{array}$

$\begin{array}{lll}12 & \text { DZ } & 42.227\end{array}$

$13 \quad \mathrm{CZ} \quad 28.727$

$\begin{array}{rrr}14 & p z & -42.379 \\ 15 & D z & 42.379\end{array}$

16 pZ 42.380

$\begin{array}{lll}\mathrm{C} & & \\ \mathrm{C} & & \end{array}$

c

$\begin{array}{lll}c & & \\ 20 & \text { px } & 28.729\end{array}$

21 px 28.729

$\begin{array}{llrccc}c & \text { Dx } & 28.729 & \text { B } & C & 0 \\ 22 & p & 0.5 & 0.8660254 & 0 & 28.730 \\ 23 & p & 0.5 & 0.8660254 & 0 & .28 .730 \\ 24 & p & -0.5 & 0.8660254 & 0 & 28.730 \\ 25 & p & -0.5 & 0.8660254 & 0 & -28.730\end{array}$

c

$\begin{array}{lll}C & & \\ 26 & p z & -42 .\end{array}$

27 D2 127.140

$\begin{array}{lll}28 & \text { p2 } & -42.3795\end{array}$

$\begin{array}{ll}\text { Volume } & \\ 29 \quad D 2 & 42.3795\end{array}$

volume

DX -350.00

$\begin{array}{lll} & \\ & \mathrm{px} & 350.00\end{array}$

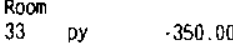

34 py $\quad 350.00$

$\begin{array}{lll}35 & \mathrm{p} 2 & -42.88 \\ 36 & \mathrm{pz} & 150.00\end{array}$

C

$\begin{array}{lll}40 & p x & -380.48 \\ 41 & p x & 380.48\end{array}$

$\begin{array}{ll}\text { py } & -380.48 \\ \text { py } & 380.48\end{array}$

Dy $\quad 380.48$

$\begin{array}{rrr}45 & \text { DZ } & -100.00 \\ & \text { DZ } & 200.00\end{array}$

Surface Definitions

Billets

Drum

(n)

The Array Boundaries

S Botton of Bottom

$s$ Top of Top Layer s Bottom of Fill

s Top of Fill

The Room

s Left Side of

s Right Side of

5 Front of Room

$S$ Back of Room

5 Floor of Room

Walls. Celing. Floor

5 l.eft wall

s Right Wall

s Front Wall

s Back Wall

s Floor

s Celing

mode $n$

$\begin{array}{lllll}\text { kcode } & 3000 & 1.0 & 10 & 100\end{array}$

$\begin{array}{llll}\mathrm{ksrC} & 0.0 & -10.0 & -33.0\end{array}$

$-12.0-35.0 \quad-33.0$

c Cemented Waste $(1.024 \mathrm{~g} / \mathrm{cc}) 65 \%$ water $\begin{array}{lll}1001.50 \mathrm{c} & -0.1092 & \mathrm{SH} \\ 8016.50 \mathrm{c} & -0.8669 & \mathrm{H}\end{array}$

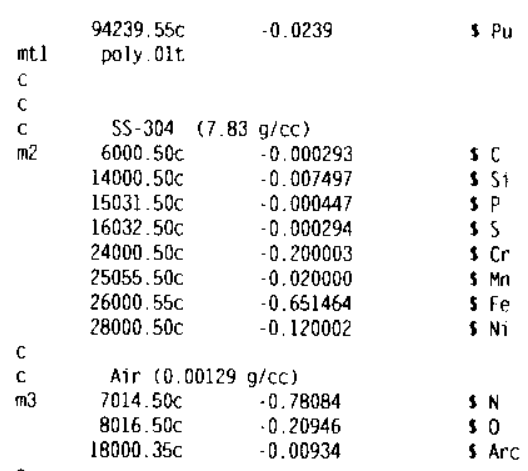

$\begin{array}{lrll}c & & \\ c & \text { Polyethylene } & (0.92 \mathrm{~g} / \mathrm{cc}) & \\ \mathrm{m} 4 & 6000.50 \mathrm{c} & 0.03952 & \\ & 1001.50 \mathrm{C} & 0.07903 & \mathrm{C} \\ & & \text { S H }\end{array}$

mt4 poly.01t

c Concrete $(2.24 \mathrm{~g} / \mathrm{cc})$

mb $1001.50 \mathrm{C} \quad .0 .009959$

$6000.50 \mathrm{C} \quad-0.000979 \quad$ S C

$8016.50 \mathrm{C}-0.529059$

$11023.50 \mathrm{C}-0.016003$

$12000.50 \mathrm{C}-0.002000 \quad-0.0006$

$3027.50 \mathrm{C}-0.034006$ S Al

$14000.50 \mathrm{C}-0.337036 \quad$ s 51

$19000.50 \mathrm{C}-0.012981 \quad$ S $K$

$20000.50 \mathrm{C} \quad-0.043965$ s $\mathrm{C}$

mt5 poly.01t of

c Water $(1.0 \mathrm{~g} / \mathrm{cc})$

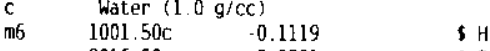

$\begin{array}{lll}8016.50 \mathrm{C} & -0.8881 & \text { s0 } 0\end{array}$

mt6 Iwtr.01t \$ Light water

c $\quad$ Cellulose $(1.45 \mathrm{~g} / \mathrm{cc})$

$\begin{array}{ll}1001.50 \mathrm{c} & 0.054311 \\ 8016.50 \mathrm{C} & 0.026949\end{array}$

$6000.50 \mathrm{C} \quad 0.03233$

mt 7 poly.01t

c Polyethylene $(0.92 \mathrm{~g} / \mathrm{cc})$

$6000.50 \mathrm{c} \quad 0.03952$

$\begin{array}{ll}6000.50 \mathrm{c} & 0.03952 \\ 1001.50 \mathrm{C} & 0.07903\end{array}$

$\begin{array}{ll}\text { mit8 } & \text { poly.01t } \\ \mathrm{c} & \text { Water }(1.02 \mathrm{~g} / \mathrm{CC})\end{array}$

c Water $(1.02 \mathrm{~g} / \mathrm{cc})$

$8016.50 \mathrm{C}-0.8703$

$\mathrm{mt}$ iwtr.01t $\mathrm{c}$ Light Water

$\mathrm{c} \quad 30 \mathrm{~g} / 1 \mathrm{Pu}$ in water $(1.03 \mathrm{~g} / \mathrm{cc})$

ml0 94239.55c $-0.0292 \quad$ S Plutoniun

$1001.50 \mathrm{c}-0,1086$ S Water Moderator

$8016.50 \mathrm{C}-0.8622$

mt10 lwtr.01t

c

totnu

ctme $\quad 480$

print

nonpipe_lat2.inp

Three Billets in a Non-pipe 55 Galion Orum

three $66.7 \mathrm{~g}$ Pu billets in a $200 \mathrm{~g} / \mathrm{drum}$

Lattice Madel

Cell Definitions

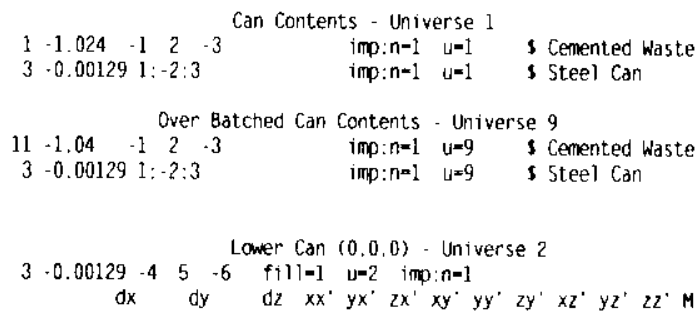




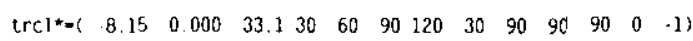

li. like 10 but 5 Can 1

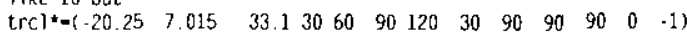

12 like 10 but

3 Can 2

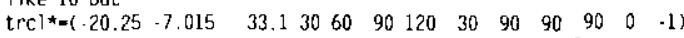
$5 \operatorname{can} 3$

c $\quad$ Lower Can (1.0.0) - Universe 3

-6 fill-1 u=3 imp: $n=1$

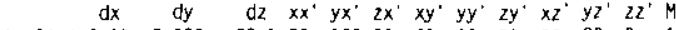

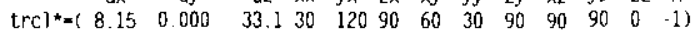

$14 \quad$ like 13 but

3 Can 1

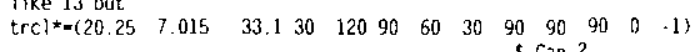

$15 \quad$ like 13 but s Can?

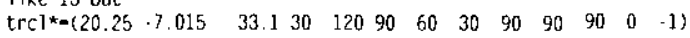
$5 \operatorname{Can} 3$

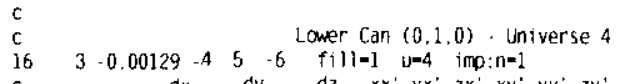

$c \quad d x \quad d y \quad d z \quad x x^{\prime} y x^{\prime} \quad z x^{\prime} \quad x y^{\prime} y y^{\prime} z y^{\prime} x z^{\prime} y z^{\prime} z z^{\prime} M$ $\operatorname{trc} 1^{*}=\left(\begin{array}{rllllllllllll}-8.15 & 0.000 & 33.1 & 90 & 180 & 90 & 0 & 90 & 90 & 90 & 90 & 0 & -1\end{array}\right)$

s Can 1

$17 \quad$ like 16 but

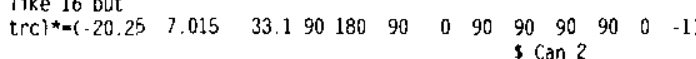

$18 \quad 1$ ike 16 but

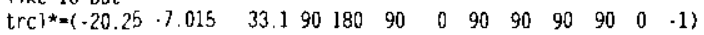
$3 \operatorname{Can} 3$

c $\quad$ Upper Can $(0.0 .0)$ - Universe 5

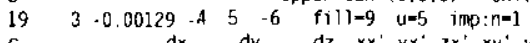

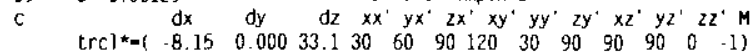

$s \operatorname{Can} 1$

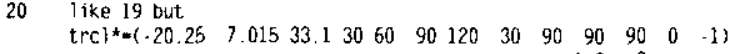

$21 \quad 1$ ike 19 but

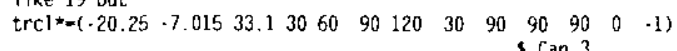

c Upper Can $(1,0.0)$ - Universe 6

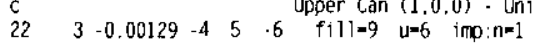

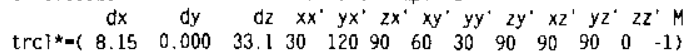

like 22 but 5 Can

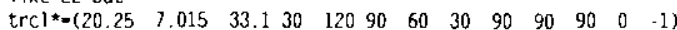

$24 \quad$ like 22 but

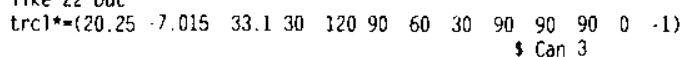

Upper Can $(0.1,0)$ - Universe ?

$25 \quad 3 \cdot 0.00129-4 \quad 5 \quad-6 \quad$ fill $=9$ is $=7$ imp:n=1

c $d x \quad d y \quad d z \quad x x^{\circ} y x^{\prime} z x^{\circ} x y^{\prime} y y^{\prime} z y^{\prime} x z^{\prime} y z^{\prime} z z^{\prime} M$

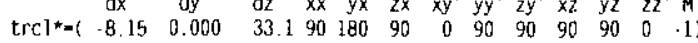

26

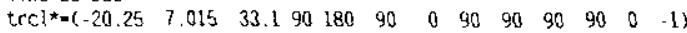

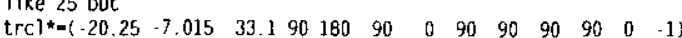
3 Can 3

Orum - Universe 2

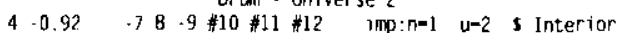

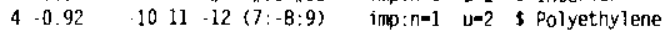

ner

$2-3.92-1314-15(10:-11: 12)$ imp:ne-1 $u=2$ s Drum

$3-0.0012913:-14: 15$ imp:n-1 $\mathrm{H}=2$ sir around Drum

Orum - Universe 3

$4-0.92 \quad-7 \quad 8 \quad 9 \quad \# 13 \quad \# 14 \quad \# 15 \quad$ inm: $n=1 \quad y=3$ s Interior

$4-0.92-1011-12\langle 7:-8: 9\} \quad$ imp: $n=1 \quad v=3$; Polyethylene

iner

$36 \quad 2 \cdot 3.92-1314-15(10:-11: 12) \quad$ imp:n=1 u=3 s Drum

$37 \quad 3-0.0012913: 14: 15$ imp:n-1 $u=3$ isir around Drum c Drum - Universe 4

$394-0.92 \quad-1011-12(7:-8: 9) \quad$ imp:n=1 $\quad \mathrm{w}=4$ s Polyethylene

$\begin{array}{llllllll}40 & 2 & -3.92 & -13 & 14 & -15 & (10:-11: 12) \quad i m p: n=1 & u=4\end{array}$ S Drum

$413-0.00129$ 13:-14:15 imo:n=1 u=4 \& Air around Drumc

c Drum - Universe 5

$42 \quad 4-0.92 \quad-78.9 \quad \# 19$ \#20 \#21 imp:n=1 u=5 s Interior

$43 \quad 4-0.92 \quad 1011-12(7:-8: 9) \quad i m p: n=1 \quad u=5$; Polyethylene

$\begin{array}{llllllllll}44 & 2 & -3.92 & -13 & 14 & -15 & (10:-11: 12) \quad i m p: n-1 & u=5 & s & \text { Drum }\end{array}$

$45 \quad 3-0.0012913:-14: 15 \quad$ imp:n=1 u=5 \& Air around Drum

$c$ Orum - Liniverse 6

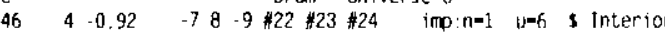

$47 \quad 4 \quad 0.92 \quad-1011-12(7:-8: 9)$ imp:n-1 u=6 solyethylene

48 ? $-3.92-1314 \cdot 15(10:-11: 12) \quad$ imp:n=1 $u=6$ s Drum

$3-0.0012913:-14: 15 \quad$ imp:n=1 $u=6$ s Air around Drum

C Drum - Universe 7

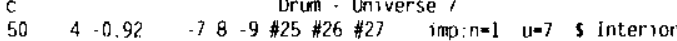

$51 \quad 4-0.92 \quad-1011-12(7:-8: 9) \quad$ imp:n*1 $u=7$ s Polyethylene

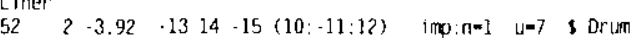

$53 \quad 3-0.0012913:-14: 15 \quad$ imo:n=1 u=7 \& Air around Drumc

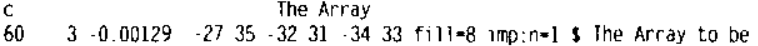
filled

Hexagonal Universe - Universe 8
$3 \cdot 0.00129 \quad-2120-2223-2425-2928$

$u=8$ lat $=2$ imp:n=1 Hex around Drum fi11--15:15 $-15: 15 \quad-1: 2$

$z=-1$

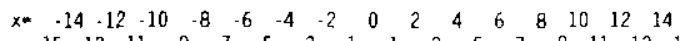
$\begin{array}{lllllllllllllllll}-15 & -13 & -11 & -9 & -7 & -5 & -3 & -1 & 1 & 3 & 5 & 7 & 9 & 11 & 13 & 15\end{array}$ 88888888888888888888888888888885

$y=-15$

$y=-14$

88 8B8888B8888888988B88888B88888

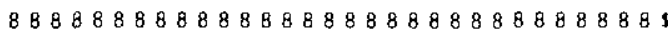

888888888868888888888888888868 s

$88888888888888 B 8888888888888888$ s

88 86888888888888888888888888888

$8 B 88888888888888888888888888888$ s

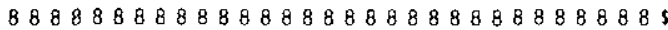

$88888888888888 B 88888688888 B 8888$ s

$88 B 888888888888888888888888888 \mathrm{~B}$ s

8888888888888888888888888888888 s

8888888888888888888888888888888 s

88888888888888888888888888888885

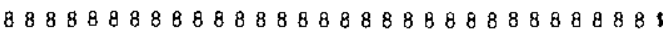

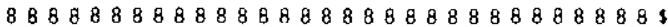

88888888888888888888888888888885

8888888888888888888 B B8888888988

$888888888888888888888888 B 888888$ s

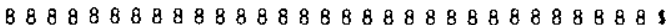

88888888888888888888888888 B B888

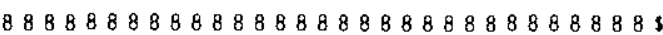

$8 B 888 B 8888888888888888868888888$ s

8888888888888888888888888888888

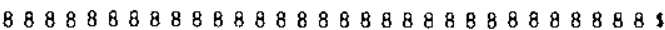

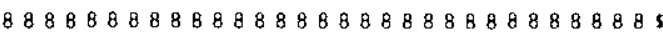


$y=10$

$y=11$

$y=12$

$y=13$

$y=14$

$y=15$

c

c

c

$y=\cdot 15$

$y=-14$

$y=-13$

$y=-12$

$y=-11$

$y=-10$

$y=-9$

$y=-8$

$y=-7$

$y=\cdot 6$

$y=-5$

$y=-4$

$y=-3$

$y=-2$

$y=-1$

$y=0$

$y=1$

$y=2$

$y=3$

$y=4$

$y=5$

$y=6$

$y=7$

$y=8$

$y=9$

$y=10$

$y=11$

$y=12$

$y=13$

$y=14$

$y=15$

c

$x=\begin{array}{lllllllllllllll}-14 & \cdot 12 & -10 & -8 & -6 & -4 & -2 & 0 & 2 & 4 & 6 & 8 & 10 & 12 & 14\end{array}$ $\begin{array}{llllllllllllllll}-15 & -13 & -11 & -9 & -7 & -5 & -3 & -1 & 1 & 3 & 5 & 7 & 9 & 11 & 13 & 15\end{array}$ $y=-15$ 888888888888888888888888888888 \& $y=-14$

88888888888888888888888888888883 8 888888888888888888888888888888 s 88BB888B8888888B888888888B88B8Bs 8888888888888888888868888888888 s $88888888888888 B 88888888888888885$ $8 B 8888 B 8888 B B B B B 888 B 88888 B 88888$ s

$2=0$

$\begin{array}{lllllllllllllll}-14 & -12 & -10 & -8 & -6 & -4 & .2 & 0 & 2 & 4 & 6 & 8 & 10 & 12 & 14\end{array}$ $\begin{array}{llllllllllllllllll}15 & -13 & -11 & -9 & -7 & -5 & -3 & -1 & 1 & 3 & 5 & 7 & 9 & 11 & 13 & 15\end{array}$ 8888888888888888888888888888888 s 8888888888B8888888888868888888

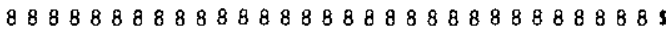
B 888888888888888888B888688888885 8888888888888888888888888888888 s 8888888888862342388888888888888 $888 B 888888884234288888888 B 888 B 8$ 88888888888234234234234234888885 888888888834234234234234238888 s B8888888882342342342342342888885 8888888888423423423423423488888 s 8888888888342342342342342388888 s 8 888888884234234234234234288888 s 8 B888888834234234234234234888885 88888888823423423423428888888885 8888888884234235634234888888888s 88888834234234274234238888888885 B 8888823423423423423428888888885 8888884234234234234238888888888 s 8888883423423423423428888888888 , 8888882342342342342348888888888 s 88888842342342342342388888888885 8888883423423423423428888888888 s 888888234234234234234888B888888s B B888842342342328888888888888885 B888883423423423888888888888888, 8888882342342342888888888888888 , 8888888884234234888888888888888 s B 888888883423423888888888888888 s 888888888234234288888688888888 B 8888888884234234888888886888888 s

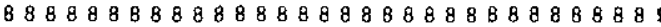

$y=13$

$y=-12$

$y=-11$

$y=\cdot 10$

$y=-9$

$y=-8$

$y=-7$

$y=-6$

$y=-5$

$y=-4$

$y=-3$

$y=-2$

$y=-1$

$y=0$

$y=1$

$y=2$

$y=3$

$y=4$

$y=5$

$y=6$

$y=7$

$y=8$

$y=9$

$y=10$

$y=11$

$y=12$

$y=13$

$y=14$ $y=15$
$C$

c

$y=15$

$y=-14$

$y=-13$

$y=12$

$y=-11$

$y=-10$

$y=-9$

$y=-8$

$y=-7$

$y=-6$

$y=-5$

$y=-4$

$y=\cdot 3$
88888888888 B8888888868888888888s

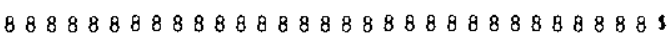
$88888888 B 8888868888888$ B 88888688 s 8 8888888888888888888888888888885 8888888888888888888888888888888 s 8888888888888888888888888888888 s 8888888888888888886888888888888 s

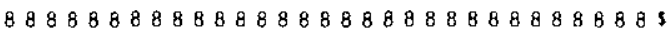
8 888888888888888888888888888 88 5 $8888888888888888888 B 88888888888$ s $8 B 888 B 888888 B 8888 B B 8 B B B 88 B B 8 B 883$

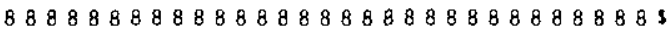
$88886888888888 B 8888888888888 B 885$ $888888888888888888888888888 B 8885$ 88888888888888888888888888888885 $888888888888888888888888 B 868888$ 8888888888888888888888888888888 s 8888888888888888888888888888888 s $8888888 B 8888888888 B 8888 B 88888885$ 8888888888888888888888888888868 s B 888868888888888888888888888888 88886888888888888888888888888885

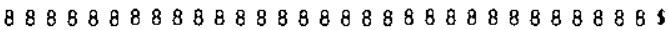
8888888888888888886888888888888 , 8888888888888888888888888886888 s 8888888888888688888888888688888 , $888888 B 88888 B 8888868888888888 B 8$ s

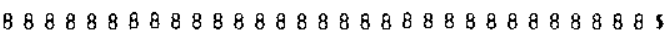

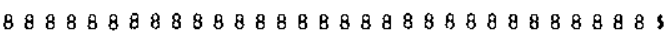

$z=2$

$\begin{array}{ccccccccccccccc}-14 & -12 & -10 & -8 & -6 & -4 & 2 & 0 & 2 & 4 & 6 & 8 & 10 & 12 & 14\end{array}$ $\begin{array}{lllllllllllllllll}15 & -13 & -11 & -9 & -7 & -5 & -3 & -1 & 1 & 3 & 5 & 7 & 9 & 11 & 13 & 15\end{array}$ 8888888888888888888888888888888 s 888 B8888B8888888888868888888888s 88888888888888888888888888888885

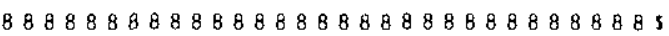
$888888888 B 8888888888888888 B 8 B 88$ s 88888888888888888888888888888883 8888888888888 8 8888888888888868 s $88888 B 888 B 888888888888888888888$ s $88888888888888888888888888 B 8888$ s $886888888888 B 8888888888888888885$ $888888888888888888888 B 8888888885$

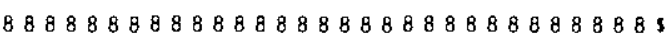
8888888888888888888888888888 B8 5 
HNF-6179 Rev. 0

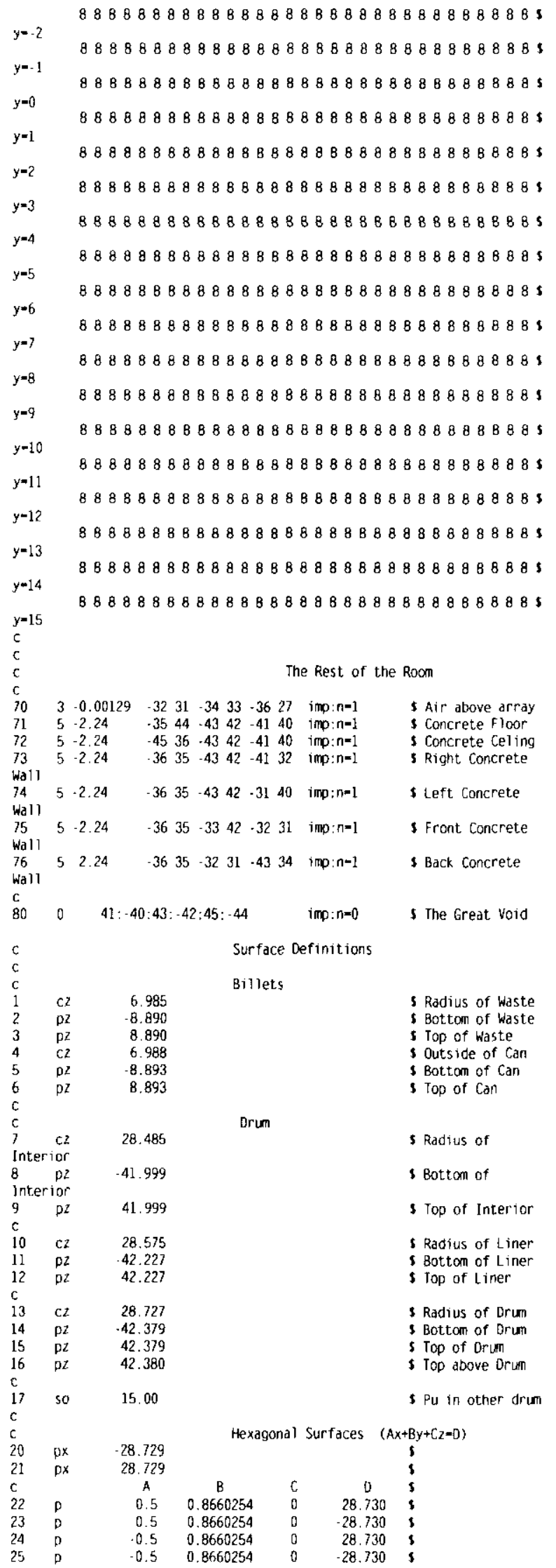

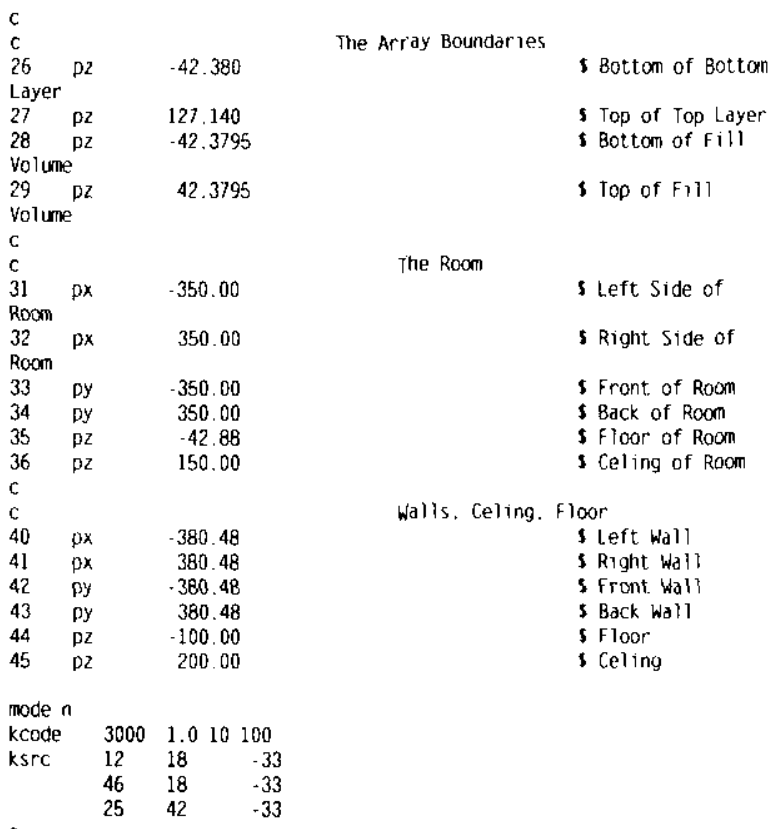

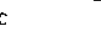

c Cemented Waste $(1.024 \mathrm{~g} / \mathrm{cc}) 100 \%$ Water

mi $\begin{array}{rrl}1001.50 \mathrm{C} & -0.1092 & 5 \mathrm{H} \\ 8016.50 \mathrm{C} & -0.8696 & 5 \mathrm{O} \\ 94239.55 \mathrm{C} & -0.0234 & 5 \mathrm{Pu}\end{array}$

mt 1 poly.01t

c

m2

$55-304 \quad(7.83 \mathrm{~g} / \mathrm{cc})$

$6000.50 \mathrm{C} \quad-0.000293 \quad$ SC

$\begin{array}{lll}14000.50 \mathrm{C} & -0.007497 & 5 \mathrm{Si}\end{array}$

$-0.000447-5$

$16032.50 \mathrm{C}-0.000294 \quad$ is

$24000.50 \mathrm{C} \quad-0.200003 \quad$ s Cr

$\begin{array}{lll}25055.50 \mathrm{C} & -0.020000 & \text { \$ Mn } \\ 26000.55 \mathrm{C} & -0.651464 & \text { S } \mathrm{Fe}\end{array}$

$28000.50 \mathrm{C} \quad-0.120002 \quad 5 \mathrm{Ni}$

c Air $(0.00129 \mathrm{~g} / \mathrm{cc})$

$\mathrm{m} 3 \quad 7014.50 \mathrm{C} \quad-0.78084 \quad$ iN

$\begin{array}{rrr}8016.50 \mathrm{C} & -0.20946 & \$ 0 \\ 18000.35 \mathrm{C} & -0.00934 & \$ \text { ArC }\end{array}$

$\begin{array}{lrrr} & 18000.35 \mathrm{c} & -0.00934 & \\ \mathrm{c} & & & \\ \mathrm{c} & \text { Polyethylene } & (0.92 \mathrm{~g} / \mathrm{cc}) & \\ \text { m4 } & 6000.50 \mathrm{c} & 0.03952 & \mathrm{~s} \mathrm{C} \\ & 1001.50 \mathrm{c} & 0.07903 & \mathrm{~s}\end{array}$

mt4 poly.01t 0.07903 is

C

$\begin{array}{lccc}\text { C } & \text { Concrete } & (2.24 \mathrm{~g} / \mathrm{CC}) \\ \text { m5 } & 1001.50 \mathrm{C} & -0.009959 & \mathrm{SH}\end{array}$

$\begin{array}{lll}1001.50 \mathrm{C} & -0.009959 & \text { SH } \\ 6000.50 \mathrm{C} & -0.000979 & \text { S C }\end{array}$

$8016.50 \mathrm{C} \quad-0.529069$ S 0

$11023.50 \mathrm{C} \quad-0.016003 \quad$ S $\mathrm{Na}$

$12000.50 \mathrm{C} \quad-0.002000 \quad \$ \mathrm{Mg}$

$14000.50 \mathrm{C}$

$14000.50 \mathrm{C}$

$\begin{array}{lll}19000.50 \mathrm{C} & -0.012981 & \$ \mathrm{~K} \\ 20000.50 \mathrm{C} & -0.043965 & 5 \mathrm{Ca}\end{array}$

$\begin{array}{rr}26000.55 \mathrm{C} & -0.014002 \text { ife } \\ \text { mit5 poly.01t } & \end{array}$

c Water $(1.0 \mathrm{~g} / \mathrm{cc})$

m6 $1001.50 \mathrm{C} \quad-0.1119 \quad$ SH

$\begin{array}{rrr}1001.50 \mathrm{C} & -0.1119 & \text { S } \\ 8016.50 \mathrm{C} & -0.8881 & \text { s }\end{array}$

mt6 Iwtr.01t s Light Water

c Celitulose $(1.45 \mathrm{~g} / \mathrm{cc})$

$\begin{array}{ll}c & \text { Ceitulose }(1.45 \mathrm{~g} / \mathrm{cc}) \\ \mathrm{m} 7 & 1001.50 \mathrm{c} \quad 0.054311\end{array}$

$8016.50 \mathrm{C} \quad 0.026949$

Sellulose Reflector

mit 7 poly.01t

c Polyethylene $(0.92 \mathrm{~g} / \mathrm{cc})$

$\begin{array}{lll}\mathrm{mB} & 6000.50 \mathrm{c} & 0.0395 \%\end{array}$

$\mathrm{mt8}$ poly.01t

S Polyethylene Reflector

\section{C-15}


HNF-6179 Rev. 0

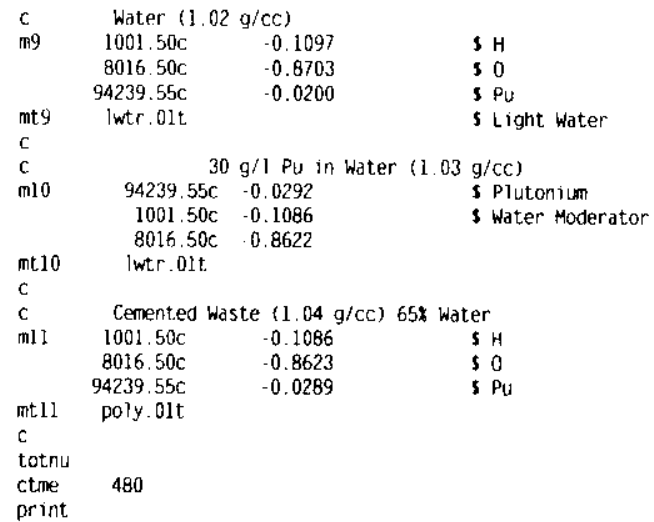

nonpipe_lat3.inp

Three Billets in a Non-pipe 55 Gallon Orum

three $66.7 \mathrm{~g} \mathrm{Pu}$ billets in a $200 \mathrm{~g} / \mathrm{dr}$

Lattice Model

Cell Definitions

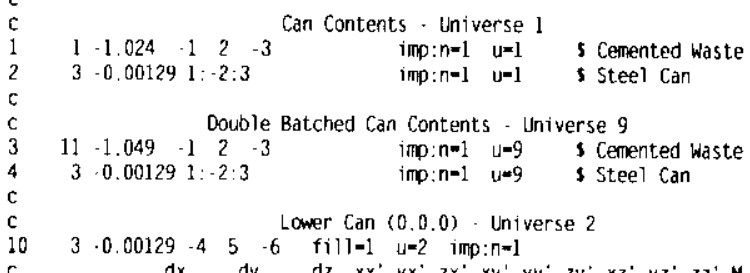

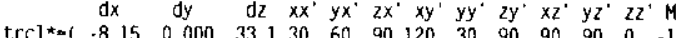

11 like 10 but

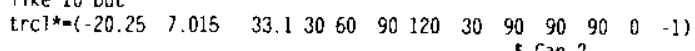

s Can 1

$12 \quad$ like 10 but

5 Can 2

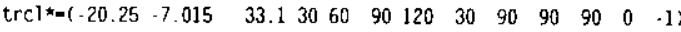
s Can 3

$\begin{array}{llllllll}\text { c } & \text { Lower Can }(1,0,0) \text { - Universe } 3\end{array}$

c $d x$ dy $d z \quad x x^{\prime} y x^{\prime} 2 x^{\prime} x y^{\prime} y y^{\prime} z y^{\prime} x z^{\prime} y z^{\prime} z z^{\prime} M$

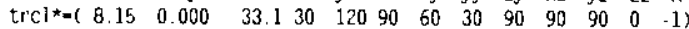

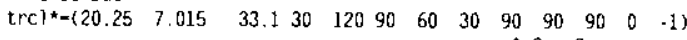

15 like 13 but

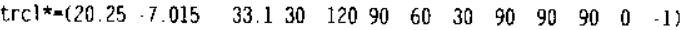
$3 \operatorname{Can} 3$

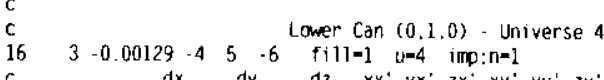

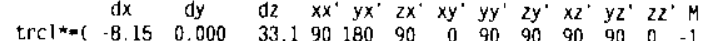

$17 \quad$ like 16 but

s Can 1

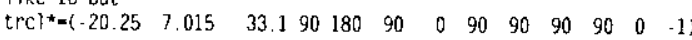

$18 \quad$ like 16 but 5 Can 2

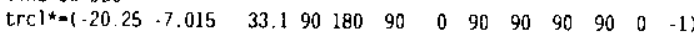

s Can 3

c Upper Can $(0,0.0)$. Universe 5

$19 \quad 3 \quad-0.00129 .4 \quad 5 \quad-6 \quad$ fill-g $\quad u=5$ imp: $n=1$

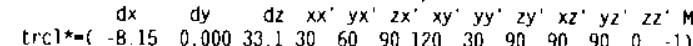

$20 \quad$ like 19 but

san 1

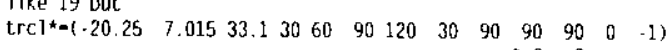

21 like 19 but

5 Can 2

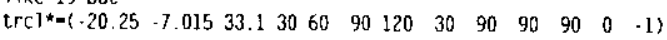

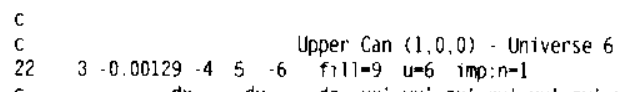

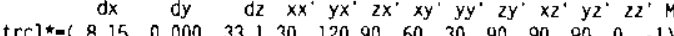

33 like 22 but \$Can 1

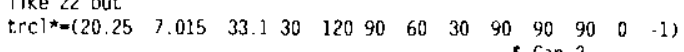

5 Can 2

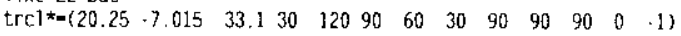
5 Can 3

$\begin{array}{lll}c & \text { Upper Can }(0,1,0) \text { - Universe } 7\end{array}$

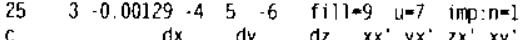

c $\quad d x \quad d y \quad d z \quad x x^{\circ} y x^{\circ} z x^{\prime} x y^{\prime} y y^{\prime} z y^{\prime} x z^{\prime} y z^{\prime} z z^{\prime} M$

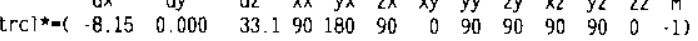

$26 \quad$ like 25 but

s Can 1

$\operatorname{trcl}^{*}=\left(\begin{array}{lllllllllllll}-20.25 & 7.015 & 33.1 & 90 & 180 & 90 & 0 & 90 & 90 & 90 & 90 & 0 & -1\end{array}\right)$

27 like 25 Dut

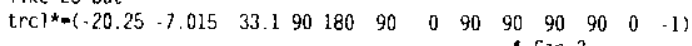

5 Can 3

C Drum - Universe 2

$30 \quad 4-0.92 \quad-7 \quad 8-9 \quad \# 10 \# 11 \# 12 \quad$ imp: $n=1 \quad u=2$ s Interior

$31 \quad 4 \cdot 0.92 \quad-1011-12(7:-8: 9) \quad$ imp:n-1 $\mathrm{u}=2$ s Polyethylene

$\begin{array}{lllllllllll}32 & 2 & -3.92 & -13 & 14 & -15 & (10:-11: 12) & \text { ind :n-1 } \quad t=2 & 5 \text { Drum }\end{array}$

$3-0.00129 \quad 13: \cdot 14: 15 \quad$ imp:n-1 $u=2$ \& Alr around Drum

c Drum - Universe 3

$34 \quad 4-0.92 \quad-78-9 \$ 13 \# 14 \# 15 \quad$ imp: $n=1 \quad u-3$ s interior

$35 \quad 4-0.92 \quad-1011 \cdot 12(7:-8: 9) \quad i m p: n \neq 1 \quad y=3$ s Polyethylene

$\begin{array}{lllllllll}36 & 2 & -3.92 & -13 & 14 & -15 & (10:-11: 12) & \text { ino:n-1 } u=3 & \text { s Drum }\end{array}$

$3-0.0012913: \cdot 14: 15 \quad$ imp:n=1 $u=3$ \& Air around Drum

c $\quad$ Drum - Universe 4

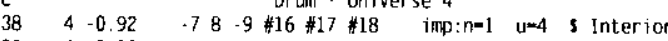

$39 \quad 4-0.92 \quad-1011 \cdot 12(7:-8: 9) \quad 1 m p: n=1 \quad u=4$ s Polyethylene

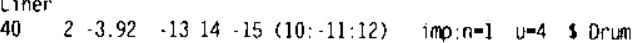

$413-0.0012913:-14: 15$ imo:n-1 u=4 s Air around Drumc

C $\quad$ Orเมn - Universe 5

$42 \quad 4 \cdot 0.92 \quad-78-9 \$ 19 \# 20$ \#21 imp:n=1 u=5 s Interior

$43 \quad 4-0.92 \quad-1011-12(7:-8: 9) \quad$ imp:n-1 $\quad u=5$ s Polyethylene

$\begin{array}{llllllllll}44 & 2 & -3.92 & -13 & 14 & -15 & (10:-11: 12) & \text { imp:n=1 u-5 s Drum }\end{array}$

$453-0.00129$ 13:-14:15 imp:n=1 $u=5$ s Air around Orum

c $\quad$ Or um - Universe 6

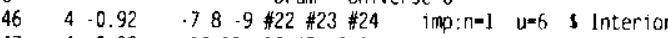

$47 \quad \begin{array}{lllll}-0.92 & -1011 \cdot 12(7:-8: 9) & i m p: n=1 & u=6 & 3\end{array}$

$48 ? \cdot 3.92-1314-15(10:-11: 12) \quad$ imp:n=1 u=6 s Drum

$493-0.0012913:-14: 15 \quad$ imp:n=1 $\|=6$ s Air around Drum

c $\quad$ Drum - Universe 7

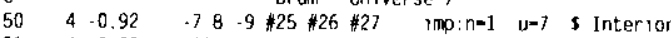

$51 \quad 4-0.92 \quad 1011-12(7:-8: 9) \quad$ imp:n=1 $\langle\neq 7$ s Polyethylene

Liner

$52 \quad 2-3.92-1314-15(10:-11: 12) \quad$ imp:n=1 $\quad u=7 \quad$ orum

$53 \quad 3-0.00129$ 13:-14:15 imp:n=1 um7 s Air around Orumc

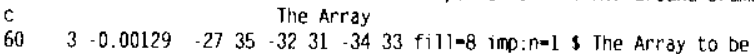
filled

c

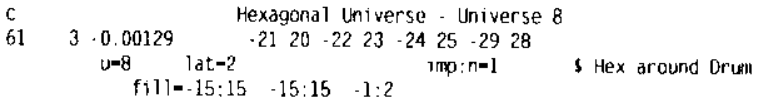

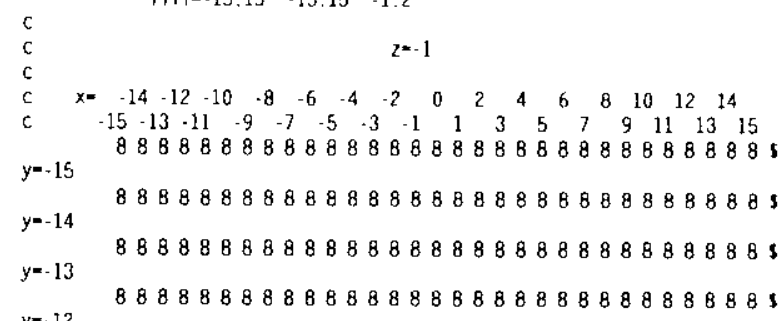


HNF-6179 Rev. 0

$y=-11$

$y=-10$

$y=-9$

$y=-8$

$y=-7$

$y=-6$

$y=-5$

$y *-4$

$y=-3$

$y=-2$

$y=-1$

$y=0$

$y=1$

$y=2$

$y=3$

$y=4$

$y=5$

$y=6$

$y=7$

$y=8$

$y=9$

$y=10$

$y=11$

$y=12$

$y=13$

$y=14$

$y=15$

c

C

c

$y=\cdot 15$

$y=-14$

$y=-13$

$y=-12$

$y=-11$

$y=-10$

$y=-9$

$y=-8$

$y=-7$

$y \rightarrow-6$

$y=-5$

$y=-4$

$y=-3$

$y=-2$

$y=-1$
88888888888888888888888888888885 $8888888 B 88888888888886888888888$ s $88 B 8888888888888898888888888888$ s $88 B 888888888868888888888$ B888888s

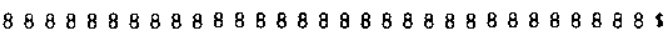
$8888888888 B 888888888888 B 8 B 888885$ $8888888888888888888888888888888 \mathrm{~s}$ $88888888 B 888888888888888$ B888888s $8888888888888888888888868888888 \mathrm{~s}$ $888888888888 B 8888888888 B 88888885$ 88 8 8 8 88888888888B88888888888885 88888888888888888888888888 B88885 88B88888888888888888888888888B85 888888888888888888888888888888 s s 8888888888888888888888888886888 s

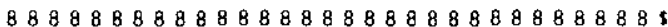

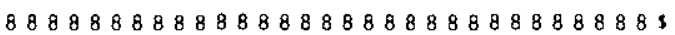
$8888888888 B 8888 B 888 B 8888 B 888888$ s $888 B 8888888888$ B88888888888888885 $8 B B 888886888888888$ B888888888888

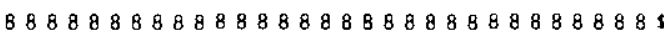
B 888888888888888888888888888888 s

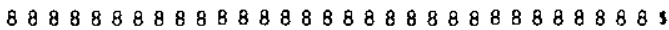
$88 B 888888888888888868$ 88 88888888 s

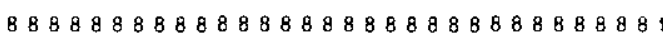
88 8 $88888888 B 8888888888688888888$ 88888888888688888888688888888885

$z=0$

$\begin{array}{lllllllllllllll}-14 & -12 & -10 & -8 & -6 & -4 & -2 & 0 & 2 & 4 & 6 & 8 & 10 & 12 & 14\end{array}$ $\begin{array}{lllllllllllllllll}15 & -13 & -11 & -9 & -7 & -5 & -3 & -1 & 1 & 3 & 5 & 7 & 9 & 11 & 13 & 15\end{array}$ 8888888888888888888888888888 888 $868888888888888888 B 88888888$ B888. $888888 B 88888888888888 B 88888888 B$ s $8888688888 B 88888 B 8888888$ B888888s 8888888888888888888688888888888

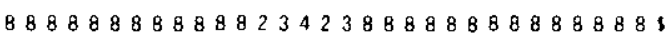
$88888888888 B 4234288888888888888$ s 888 B B 8888B823423423423423488888 8888888888342342342342342388888

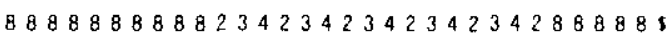
888 B 888888423423423423423488888 8888888888342342342342342388888 s 8888888884234234234234234288888 s 8888888883423423423423423488888 , 8888888882342345342342888888888 s $y=0$

$y=1$

$y=2$

$y=3$

$y=4$

$y=5$

$y=6$

$y=7$

$y=8$

$y=9$

$y=10$

$y-3]$

$y=12$

$y=13$

$y=14$

$y-15$$$
\text { C }
$$

$$
\text { C }
$$$$
\text { c }
$$$$
y=-15
$$

$y=-14$

$y=-13$

$y=-12$

$y=-11$

$y=-10$

$y=\cdot 9$

$y=-8$

$y=-7$

$y=-6$

$y=-5$

$y=-4$

$y=-3$

$y=-2$

$y=-1$

$y=0$

$y=1$

$y=2$

$y=3$

$y=4$

$y=5$

$y=6$

$y=7$

$y=8$

$y=9$

$y=10$
8888888884234234234234888888888 8888883423423423423423888888888 s 888888234234234234234288888 B B885 8888884234234234234238888888888 s $88 B 88834234234234234288888888885$ 88888823423423423423488888888885 $88888842342342342342388888 B 8888$ s 8888883423423423423428888888888 s 88888823423423423423488888888885 88868842342342328888888888888885 88888834234234238888888888888883 8888882342342342888888888888888 s 8888888884234234888888888888888 s $8888888883423423888888888888888 \mathrm{~s}$ 8888888882342342888888888888 B88s 88888988842342348888888888888884

$2-1$

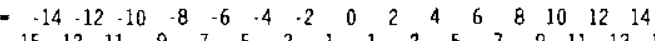
8888888888888 $8888888 B 8888888888688888 B 8888 B 85$ $8888888888888888886888886888888 \mathrm{~s}$

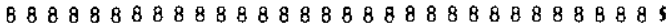

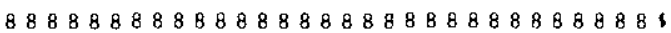
8888888888888888888888888888888 s $8 B 88888888888888 B 88888888888 B 885$ 8888888888888888888886888888888 s 8888888888888888888888888888888 s

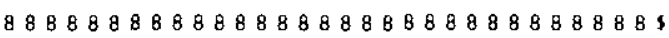
$888888 B 88888 B 888888888888888888$ s 8888888888888888888868888888888 s

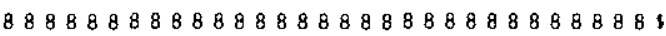
$888888 B 888888888888888888888888$,

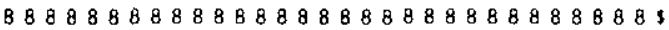
B8888868888888888888868888888885

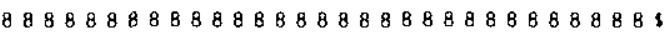

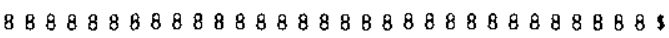
88888888888888888888888888888885

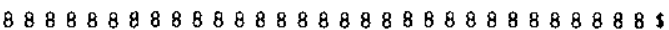
$8888888888888888888888888888888 \mathrm{~s}$ $8 B 888888888 B 88886888888888888 B 8$ s B88888888B88888B88888888888888Bs 8888888888888888888888888888888 s

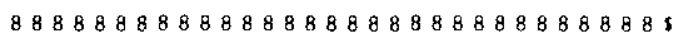
$88688888888888888888 B 88888888885$ 
HNF-6179 Rev. 0

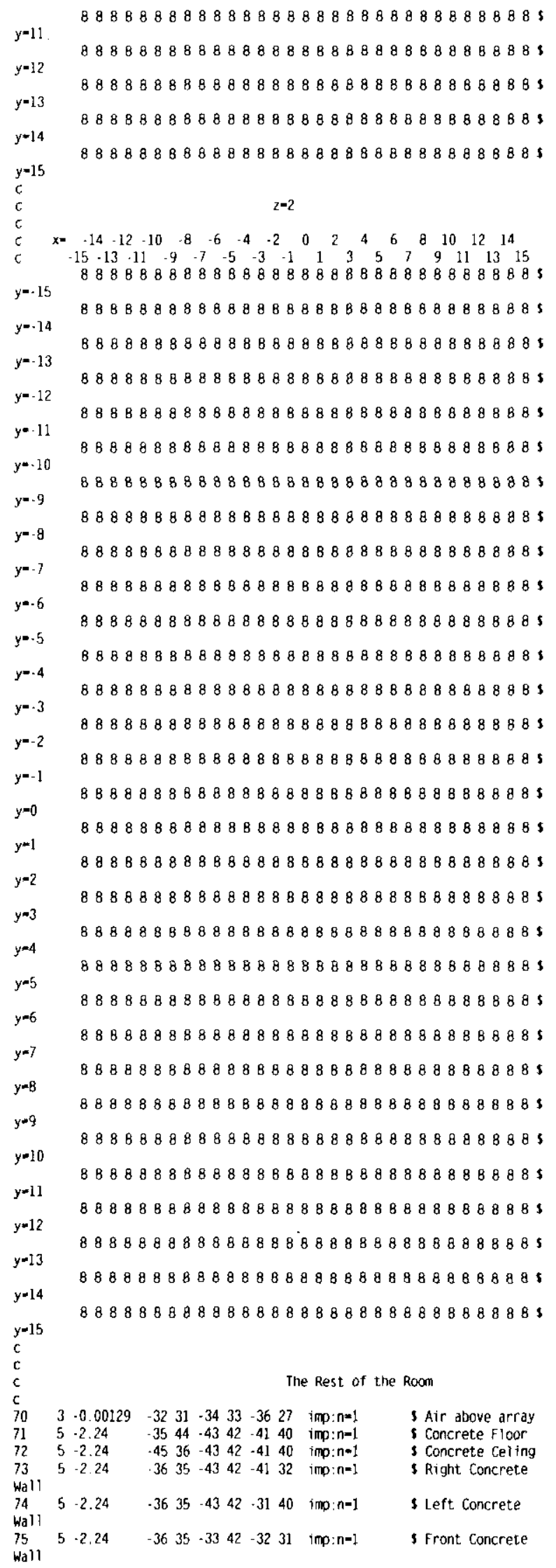

\begin{tabular}{|c|c|c|c|c|c|}
\hline $\begin{array}{l}76 \\
\text { Wall }\end{array}$ & $5-2$ & $\begin{array}{llll}-36 & 35 & -32 & 31\end{array}$ & $1-43 \quad 34$ & $\operatorname{imp}: n=1$ & I Back Concrete \\
\hline $\begin{array}{l}c \\
80\end{array}$ & 0 & $41:-40: 43:-42: 45:-4$ & 44 & imp: : $1=0$ & 5 The Great Void \\
\hline $\begin{array}{l}c \\
c\end{array}$ & \multicolumn{5}{|c|}{ Surface Definitions } \\
\hline c & & & Billets & & \\
\hline 1 & $c z$ & 6.985 & & & 5 Radius of Waste \\
\hline 2 & $p Z$ & .8 .890 & & & S Botton of Waste \\
\hline 3 & $p z$ & B. 890 & & & S Top of Waste \\
\hline 4 & $c z$ & 6.988 & & & S Outside of Can \\
\hline 5 & $\rho 2$ & -8.893 & & & \$ Bottom of Can \\
\hline 6 & $\rho z$ & 8.893 & & & \$ Top of Can \\
\hline c & & & & & \\
\hline c & & & Drum & & \\
\hline 7 & $c z$ & 28.485 & & & s Rađius of \\
\hline Inter & & & & & \\
\hline $\begin{array}{l}8 \\
\text { Inter }\end{array}$ & pz & -41.999 & & & $s$ Bottom of \\
\hline $\begin{array}{l}9 \\
c\end{array}$ & $p 2$ & 41.999 & & & s Top of Interior \\
\hline 10 & $c z$ & 28.575 & & & S Radius of $\mathrm{t}$ iner \\
\hline 11 & $p z$ & -42.227 & & & s Bottorn of Liner \\
\hline 12 & pz & 42,227 & & & $\$$ Top of Liner \\
\hline $\begin{array}{c}c \\
13\end{array}$ & $c z$ & 28.727 & & & S Radius of Drum \\
\hline 14 & $p z$ & .42 .379 & & & 5 Botton of Drum \\
\hline 15 & Dz & 42.379 & & & s Top of Drum \\
\hline 16 & D2 & 42.380 & & & 5 Top above Drum \\
\hline $\begin{array}{c}C \\
17\end{array}$ & & & & & 3. Pu in ather orun \\
\hline
\end{tabular}


HNF-6179 Rev. 0

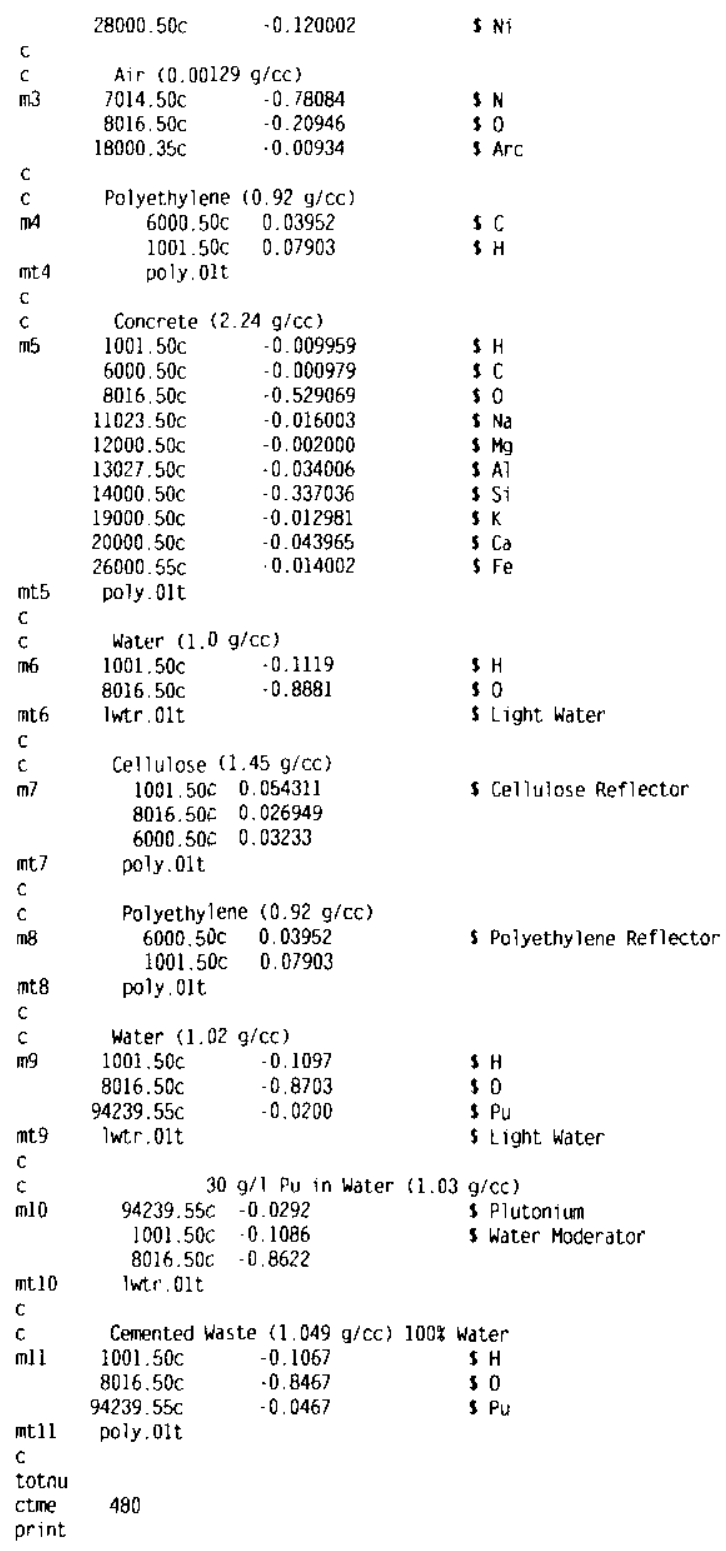

nompipe_lat3a.1np

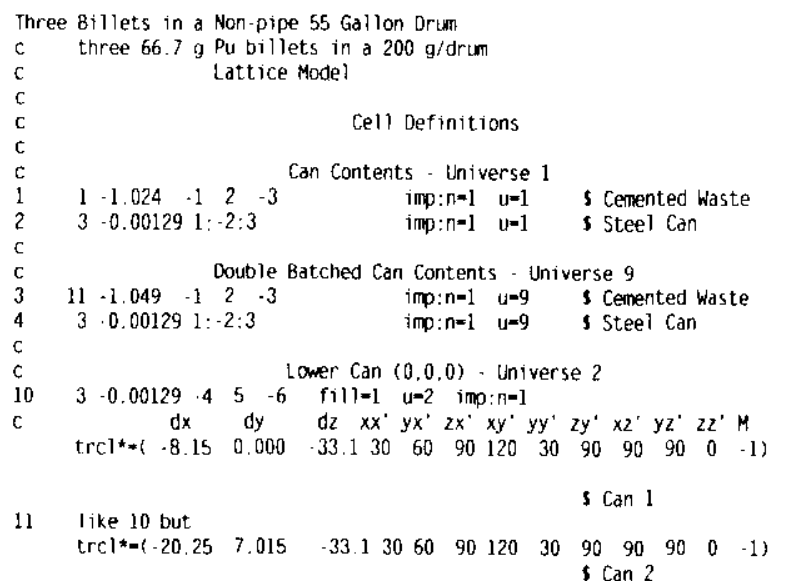

12 like 10 but.

$\left.\begin{array}{lllllllllllll}\operatorname{trc} 1^{*}-(-20.25 & -7.015 & -33.1 & 30 & 60 & 90 & 120 & 30 & 90 & 90 & 90 & 0 & -1\end{array}\right)$

c Lower Can $(1,0,0)$. Universe 3

$13 \quad 3-0.00129-4 \quad 5 \quad 6 \quad$ fill-1 $\quad \mathrm{y}=3$ imp:n-1

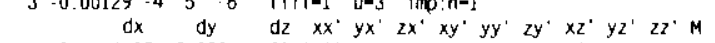

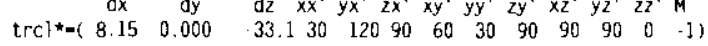

14 like 13 but 3 Can 1

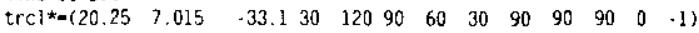

$5 \operatorname{can} 2$

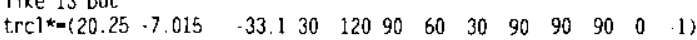
$5 \operatorname{can} 3$

$3-0.00129-4 \quad 5 \quad$ Lower Can $(0,1,0)$ - Universe 4 $\begin{array}{llll}-6 & \text { fill }=1 \quad u=4 & \text { imp: } n=1 \\ -4 & 5 & 0\end{array}$

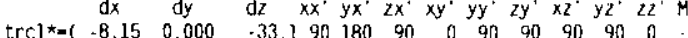

1)

like 16 but

s Can 1

$\begin{array}{llllllllllll}\operatorname{trcl}^{*}-(-20.25 & 7.015 & -33.1 & 90 & 180 & 90 & 0 & 90 & 90 & 90 & 90 & 0\end{array}$

like 16 but

s Can 2

$\begin{array}{llllllllllll}\operatorname{trc})^{*}=(-20.25 & -7.015 & -33.1 & 90 & 180 & 90 & 0 & 90 & 90 & 90 & 90 & 0\end{array}$

$5 \operatorname{can} 3$

$3-0.00129-4 \quad 5 \quad-6 \quad$ fi $T 1=9$ Upper $(0.0 .0)$ - Universe 5

$d x \quad d y \quad d z \quad x x^{\prime} y x^{\prime} z x^{\prime} x y^{\prime} y y^{\prime} z y^{\prime} x z^{\prime} y z^{\prime} \quad z z^{\prime} M$

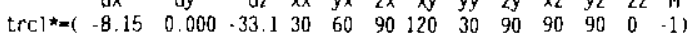

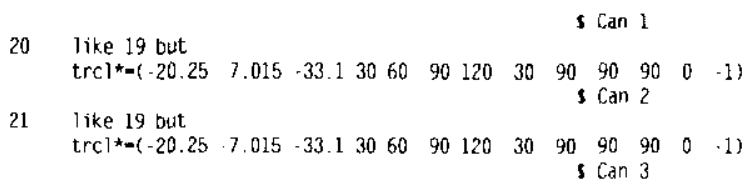

$\begin{array}{llllll} & -0 & 0 & \text { Upper Can }(1,0.0) \text { - Universe } 6\end{array}$

$\begin{array}{llllll}3-0.00129 & -4 & 5 & -6 & \text { fill-9 } \quad u=6 & \text { imp: } n=1\end{array}$

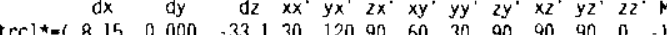

like 22 but 5 Can 1

like 22 but

$90 \quad 90 \quad 90 \quad 00-1)$

$24 \quad$ like 22 put $5 \operatorname{can} 2$

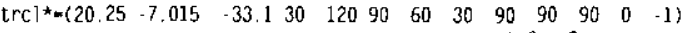

$5 \operatorname{can} 3$

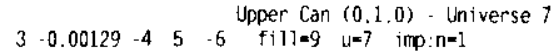

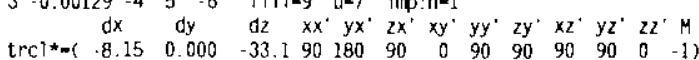

s [an $]$

26

1ike 25 but

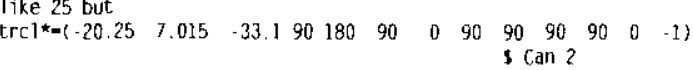

$27 \quad$ like 25 but $\left.\begin{array}{lllllllllllll}\operatorname{trc}]^{*}=(-20.25 & -7.015 & -33.1 & 90 & 180 & 90 & 0 & 90 & 90 & 90 & 90 & 0 & -1\end{array}\right)$

Drum - Universe 2

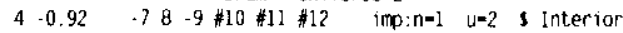

$\begin{array}{lllll}4 & -0.92 \quad-10 & 11-12(7:-8: 9) \quad \text { imp: } n=1 & u=2 & 5\end{array}$

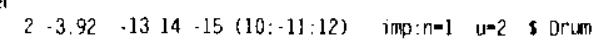

3-0.00129 13:-14:15 imp:n=1 u-2 s Air around Drum

Drum - Universe 3

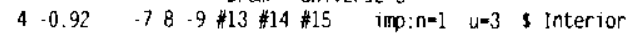

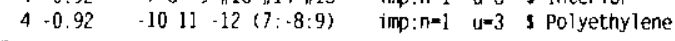

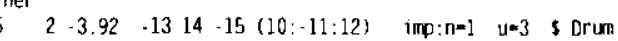

$3-0.0012913:-14: 15 \quad$ imp:n*1 u=3 s Air around Drum

Drum - Universe 4

$4 \cdot 0.92 \quad-78 \cdot 9 \# 16 \# 17 \# 18 \quad$ imp:n=1 $u=4$ s Interior

$\begin{array}{lllll}4-0.92 & -1011 \cdot 12(7:-8: 9) & \text { imp:n-1 } & u=4 & \text { Interior }\end{array}$ 
HNF-6179 Rev. 0

\begin{tabular}{llll}
40 & 2 & -3.92 & $-1314-15(10:-11: 12)$ \\
41 & $3 \cdot 0.00129$ & $13:-14: 15$ & imp:n \\
$\mathrm{c}$ & \multicolumn{4}{c}{ Drum . Universe 5} \\
$\mathrm{C}$ &
\end{tabular}

$42 \quad 4 \cdot 0.92 \quad-78-9 * 19 * 20$ \#21 imp:n-1 u=5 s Interior

$43 \quad 4-0.92 \quad-1011-12(7:-8: 9) \quad i m p: n-1 \quad u=5$ s Polyethylene

$\begin{array}{llllllll}\text { Liner } & 2 & -3.92 & -13 & 14 & -15(10:-11: 12) & \text { imp:n-1 } u-5 & \text { s Drum }\end{array}$

$45 \quad 3-0.00129$ 13:-14:15 imp:n=1 uns I Air around Drum

c Drum - Universe 6

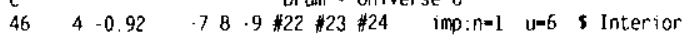

$47 \quad 4-0.92 \quad-1011 \cdot 12(7:-8: 9) \quad m: n: n-1 \quad u=6$ s Polyethylene

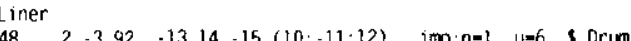

$49 \quad 3-0.0012913:-14: 15$ imp:n=1 $u=6$ s Air around Drum

C $\quad$ Drum - Universe 7

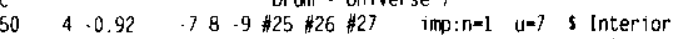

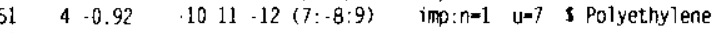

Liner $2 \cdot 3.92 .1314-15(10 \cdot-11: 12) \quad$ imo:n-1 $y=$ ? $s$ Drum

$53 \quad 3-0.0012913:-14: 15$ imp:n-1 $u=7$ s Air around Drumc

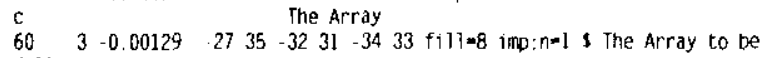
filled

$c$
6
61

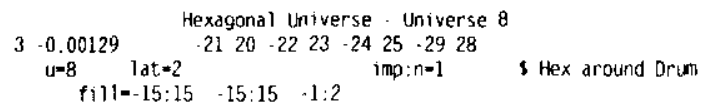

$c$
$c$
$c$
$c$

$x=\begin{array}{lllllllllllllll}-14 & -12 & -10 & -8 & -6 & -4 & -2 & 0 & 2 & 4 & 6 & 8 & 10 & 12 & 14\end{array}$ $\begin{array}{llllllllllllllll}-15 & -13 & -11 & -9 & -7 & -5 & -3 & -1 & 1 & 3 & 5 & 7 & 9 & 11 & 13 & 15\end{array}$ 8888 B 88888888888888888888888888,

$y=-15$

$y=-14$

$y=-13$

$y=-12$

$y=-11$

$y=-10$

$y=-9$

$y=-8$

$y=.7$

$y=-6$

$y=-5$

$y=-4$

$y=-3$

$y=-2$

$y=-1$

$y=0$

$y=1$

$y-2$

$y=$

$y=3$

$y=4$

$y=5$

$y=6$

$y=7$

$y=8$

$y=9$

$y=10$

$y=11$
888888888888888888888888888888 s 88888888888888888888888888888885 B 8888B88888888888888868888888885 $888 B 888888 B 88888888888888888888$ s $8888888888888888886888888888888 \mathrm{~s}$ B8888888888888888888888888888885

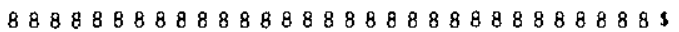
888888888888888888888888 B8888B8 88888888888888888888888888888885 88888888888888888888888888888885 $888888888 B 8888988888 B 8888889888$ s 8888888888888888888888888888888 s

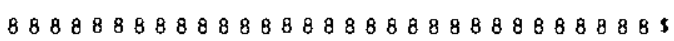
8888888888688888888888888888888 s 88888888888888888888888888888885

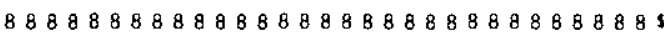

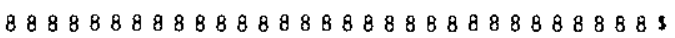
$8 B 88888888888$ B B88888888B8888B885 $888888888888888888888888888888 B$

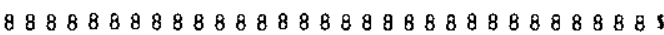

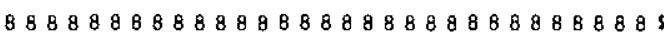
88 888888888888888888888888888885 8888888888888888888888888888888 8888888888888888888888888888888 s B 888888888888888888888888888888 $888868888 B 868888888888888 B 88888$ s $y-12$

$y=13$

$y=14$

$y=15$

C

c

$$
\text { c }
$$

$y=-15$

$y=-14$

$y=-13$

$y=-12$

$y=-11$

$y=-10$

$y=-9$

$y=-8$

$y=-7$

$y=-6$

$y=-5$

$y=-4$

$y=-3$

$y=-2$

$y=-1$

$y=0$

$y-1$

$y=2$

$y=3$

$y=4$

$y=5$

$y=6$

$y=7$

$y=8$

$y=9$

$y=10$

$y=11$

$y=12$

$y=13$

$y=14$

$y=15$$$
\text { c }
$$$$
c
$$$$
\text { c }
$$

$y=-15$

yn $\cdot 14$

$y=-13$

$y=-12$
$8888888888888888888888888888888 \mathrm{~s}$ $88888888 B 888888888888888 B 888888$ s

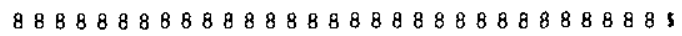
$888888 B 888888888888888888 B 88888$ s

\section{$z=0$}

$\begin{array}{lllllllllllllll}-14 & -12 & -10 & -8 & -6 & -4 & -2 & 0 & 2 & 4 & 6 & 8 & 10 & 12 & 14\end{array}$ $\begin{array}{llllllllllllllll}15 & -13 & -11 & -9 & -7 & -5 & -3 & -1 & 1 & 3 & 5 & 7 & 9 & 11 & 13 & 15\end{array}$ 888888888888888 8 888 888888688888

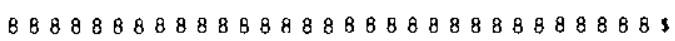
8888888888888888888888888888888 s $88888688888888888888888 B 8888868$ s

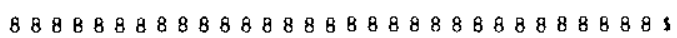
8888888888882342388888888888888 s 8888888888884234288888888888888 s $8 B 8888 B 888823423423423423488888$ s 8888888888342342342342342388888 s 8886888888234234234234234286888 s 8888888888423423423423423488888 s B888888888342342342342342388888s 8888888884234234234234234288888 s 8888888883423423423423423488888 s $888888 B 8823423453423428 B 8888888$ s 8888888884234234234234888888888 s 8888883423423423423423888888888 s 8888882342342342342342888888888 s 8888884234234234234238888888888 s 8888883423423423423428888888888 s 8888882342342342342348888888888 , 8888884234234234234238888888888 s 88888834234234234234288888888883 $888888234234234234234888 B 888888$, $8688884234234232868888868888 B 885$ 88888834234234238888888888888885 88888823423423428888888888888885 $8888888 B 842342348 B 88888888888 B 8$ s 8888888883423423888888888888888 s 88 88888882342342888888888B88888, $888888 B 8842342348 B 8888888888888$ s

$2=1$

$\begin{array}{llllllllllllllll}- & -14 & -12 & -10 & -8 & -6 & -4 & -2 & 0 & 2 & 4 & 6 & 8 & 10 & 12 & 14\end{array}$ $\begin{array}{llllllllllllllll}15 & -13 & -11 & -9 & -7 & -5 & -3 & -1 & 1 & 3 & 5 & 7 & 9 & 11 & 13 & 15\end{array}$ B88888888888888868888886888888Bs $8888888888888888888 B 8886 B 8888 B B$ s 88888888888888888888888888888885 8888888888888888888888888888888 s 
HNF-6179 Rev. 0

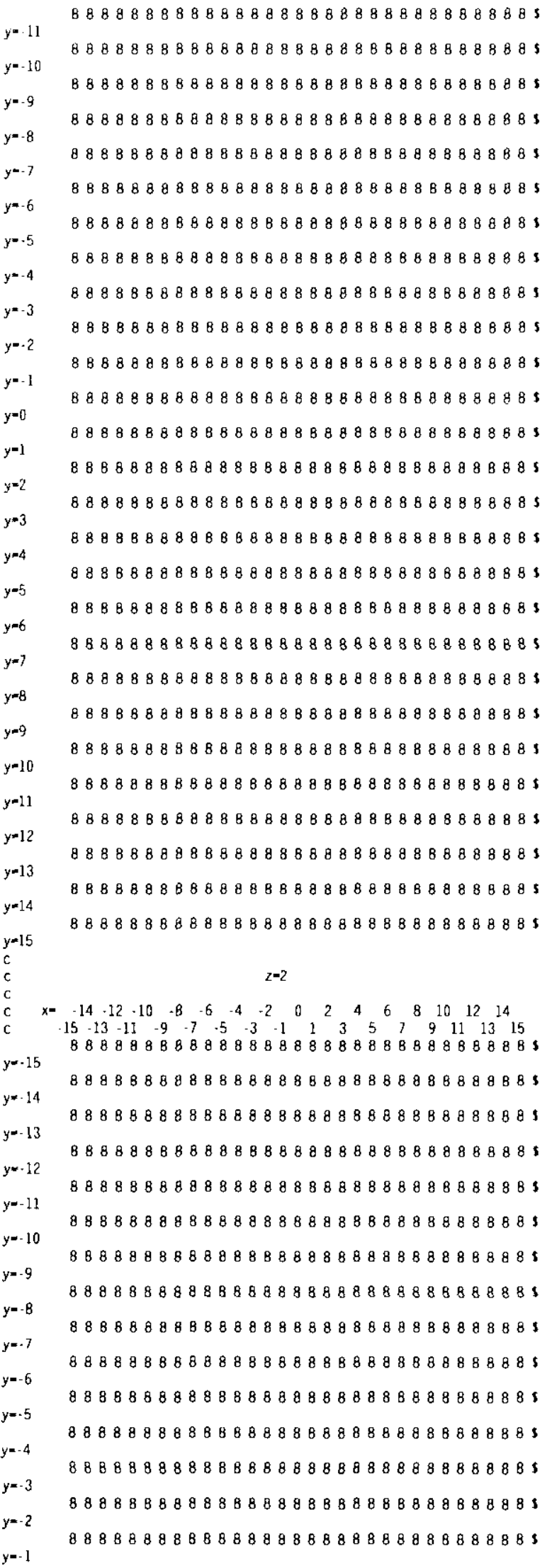

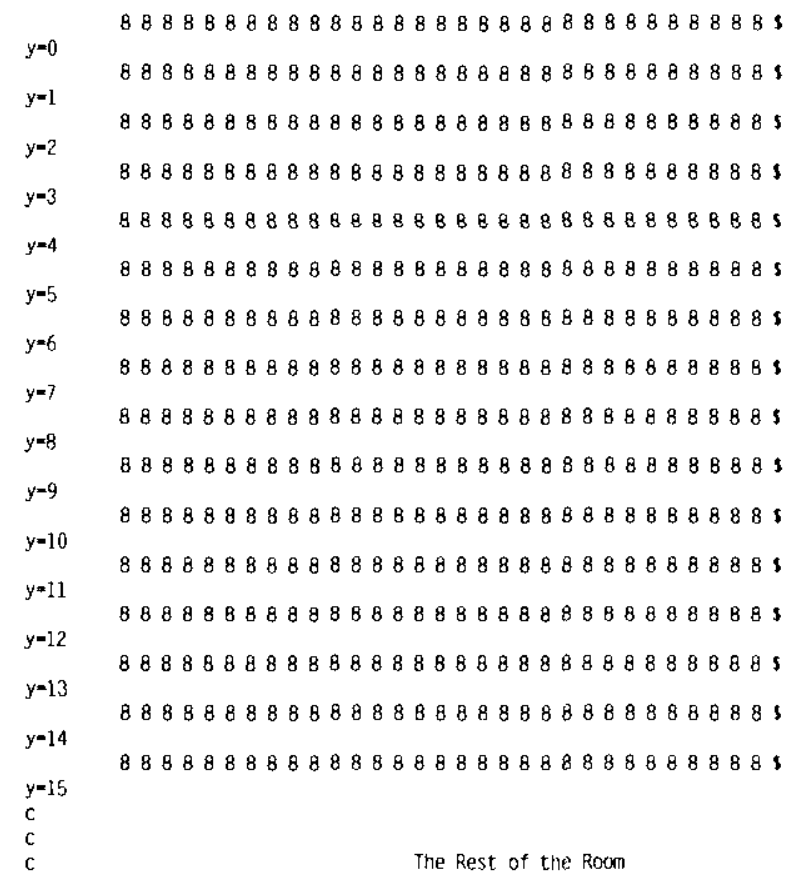

$\begin{array}{lllllllll}3 & -0.00129 & -32 & 31 & -34 & 33 & -36 & 27 & \text { imp: } n=1\end{array}$

$\begin{array}{llllllll}5 & -2.24 & -35 & 44 & -43 & 42 & -41 & 40\end{array}$ imo: $n=1$

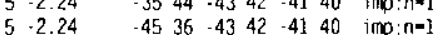

$\begin{array}{lllllllll}5 & -2.24 & -45 & 36 & -43 & 42 & -41 & 40 & \text { imp: }: n=1 \\ 5 & -2.24 & -36 & 35 & -43 & 42 & -41 & 32 & \text { imp:n=1 }\end{array}$

Wall

$\begin{array}{lll}74 & 5 & -2.24\end{array}$

$75 \quad 5 \cdot 2.24$

Wa 1

Wal

c
5 Air above drray

S Concrete Floor Soncrete Celing $\checkmark$ Right concrete

$s$ Left Concrete

s Front Concrete

s Back Concrete

s The Great vord Surface Definitions Billets

Sadius of Waste Bottom of Waste Top of Waste s Outside of Can s Bottom of can s Top of Can

5 Radius of

S Bottom of

s Top of Interior

S Radius of Liner 5 Bottom of Liner $\checkmark$ Top of Liner

5 Radius of Drum 5 Bottom of Drum s Top of Drum

$s$ Top above Drum

s Pu in other arum

Hexagonal Surfaces $(A x+B y+C z-D)$

$$
.28 .729
$$$$
28.729
$$$$
\begin{array}{cc}
\text { A } & \text { B } \\
0.5 & 0.8660254
\end{array}
$$$$
\begin{array}{ll}
0.5 & 0.8660254 \\
0.5 & 0.8660254
\end{array}
$$$$
\begin{array}{ll}
-0.5 & 0.8660254 \\
-0.5 & 0.8660254
\end{array}
$$$$
\begin{gathered}
\text { Surfaces } \quad(A x+B \\
0 \\
28.730 \\
-28.730 \\
28.730 \\
-28.730
\end{gathered}
$$$$
\begin{aligned}
& 5 \\
& 5 \\
& 5 \\
& 5
\end{aligned}
$$

The Array Boundaries

$-42.380$

s Botton of Botton 
HNF-6179 Rev. 0

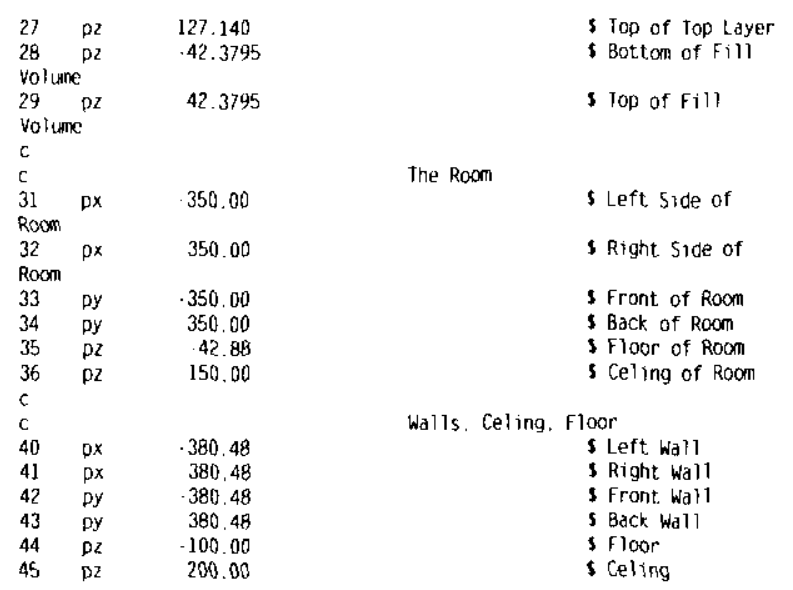

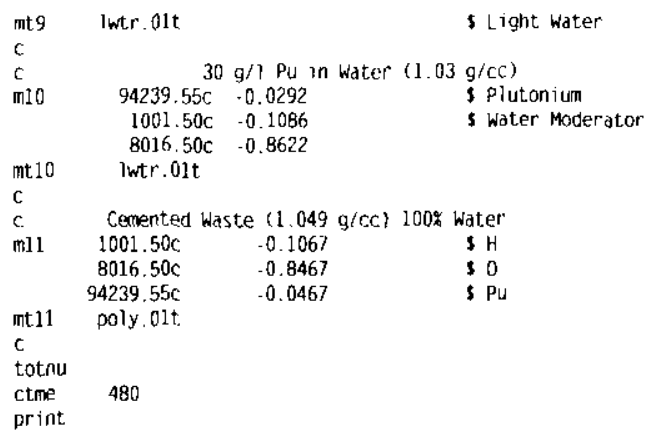

mode $n$

kcode $\quad 3000 \quad 1.010100$

$\begin{array}{lllll}\text { ksrc } \quad 0.0 & \cdot 10.0 & 33.0\end{array}$

$12.0-35.0 \quad 33.0$

$12.0 \quad 35.0 \quad 33.0$

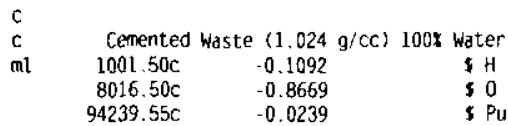

mtl poly.01t

$\mathrm{H}$
$\mathrm{P}$
$\mathrm{Pu}$

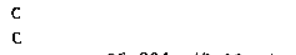

$\begin{array}{lccc}c & 55.304 & (7.83 \mathrm{~g} / \mathrm{cc}) & \\ \mathrm{m} 2 & 6000.50 \mathrm{C} & 0.000293 & \text { s } \mathrm{C}\end{array}$

$14000.50 \mathrm{C} \quad-0.007497 \quad$ s $5 \mathrm{Si}$

$15031.50 \mathrm{c} \quad-0.000447$ is

$16032.50 \mathrm{C}-0.000294$ is

$24000.50 \mathrm{C}-0.200003 \quad \mathrm{~s} \mathrm{Cr}$

$25055.50 \mathrm{C} \quad-0.020000 \quad$ s Mn

$\begin{array}{lll}26000.55 \mathrm{C} & -0.651464 & 5 \mathrm{Fe} \\ 28000.50 \mathrm{C} & -0.120002 & 5 \mathrm{Ni}\end{array}$

c $\quad$ Air $(0.001299 / \mathrm{cc})$

m3 7014.50c $\quad-0.78084$ S N

$\begin{array}{rrr}8016.50 \mathrm{C} & -0.20946 & \text { S } 0 \\ 28000.35 \mathrm{C} & -0.00934 & \text { S ArC }\end{array}$

$\begin{array}{lrl}c & & \\ c & \text { Polyethyiene }(0.92 \mathrm{~g} / \mathrm{cc}) & \\ \mathrm{m} 4 & \text { 5000.50C } 0.03952 & \text { s } \mathrm{C} \\ & 1001.50 \mathrm{C} \quad 0.07903 & \text { s } \mathrm{H}\end{array}$

mt4 poly.01t

c $\quad$ Concrete $(2.24 \mathrm{~g} / \mathrm{cc})$

c Concrete $(2.24 \mathrm{~g} / \mathrm{cc})$

$\begin{array}{ll}1001.50 \mathrm{C} & -0.009959 \\ 6000.50 \mathrm{C} & -0.000979\end{array}$

$8016.50 \mathrm{C} \quad-0.529069$

$11023.50 \mathrm{C}$

$12000.50 \mathrm{C}$

$13027.50 \mathrm{c} \quad .0 .034006$

$14000.50 \mathrm{C} \quad-0.337036$

$19000.50 \mathrm{C} \quad .0 .012981$

$20000.50 \mathrm{c} \quad-0.04396$

$26000.55 \mathrm{c}$

poly.01t

$-0.014002 \quad \$ \mathrm{Fe}$

c Water $(1.0 \mathrm{~g} / \mathrm{cc})$

m6 $1001.50 \mathrm{C}-0.1119 \quad$ s $\mathrm{H}$

$8016.50 \mathrm{C}-0.8881 \quad 30$

mt6 iwte.01t S Light water

c Cellulose $(1.45 \mathrm{~g} / \mathrm{cc})$

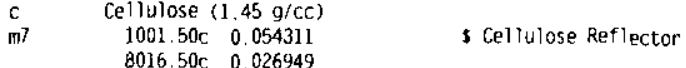

$6000.50 \mathrm{C} \quad 0.03233$

int 7 poly. $01 \mathrm{t}$

c Polyethylene $10.92 \mathrm{~g} / \mathrm{cc})$

and $\quad 6000.50 \mathrm{c} \quad 0.03952$

$\begin{array}{ll}1001.50 \mathrm{C} & 0.07903\end{array}$

mt8 poly olt

5 Polyethylene Reflector

Water $(1.02 \mathrm{~g} / \mathrm{cc})$

$\begin{array}{lll}1001.50 \mathrm{C} & 0.1097 & 5 \mathrm{H} \\ 8016.50 \mathrm{C} & 0.8703 & 50\end{array}$

$\begin{array}{rrr}94239.55 \mathrm{C} & -0.0200 & 5\end{array}$

nonpipe_lat3b. Inp

Three Billets in a Nan-pipe 55 Gallon Orun

three $66.7 \mathrm{~g}$ Pu billets in a $200 \mathrm{~g} / \mathrm{dr}$ Lattice Model

Cell Definitions

Can Contents - Universe 1

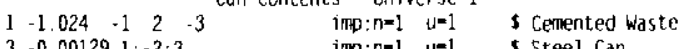

$3-0.001291:-2: 3 \quad$ imp:n-1 $u=1$ steel Can

Double Batched Can Contents - Universe 9

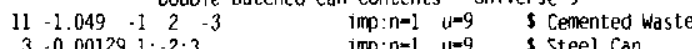

3. -0.00129 1:-2:3 imp: $n=1 \quad u=9$ s Steel Can

Lower Can $(0.0 .0)$ - Universe ?

$3-0.00129-4 \quad 5 \quad-6 \quad$ fill $-1 \quad u=2$ imo: $n=1$

$\operatorname{trcl}=\left(\begin{array}{ccc}d x & d y & d z \\ 7.0581 & 4.075 & -33.1) \quad \text { S Can } 1\end{array}\right.$

like 10 but

$\operatorname{trcl}=\left(\begin{array}{lll}14.0295 & 16.200 & -33.1\end{array}\right) \quad 5 \mathrm{Can} 2$

like 10 but

s Can 3

$3.0 .00129-45$ Lower Can $(1,0,0)$ - Universe 3

$\begin{array}{llllllll}3 & -0.00129 & -4 & 5 & -6 & f i] l=1 & u-3 & \text { imp: } n=1\end{array}$

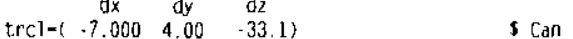

like 13 but

trcil- $-21.04454 .0498-33.1) \quad$ San 2

15. like 13 but

$\operatorname{trcl}=(-14.0295(6.200-33.1) \quad$ s can 3

$3-0.00129-45$ Lower Can $(0,1,0)$ - Universe 4

$\operatorname{trcl}=\left(\begin{array}{ccc}d x & d y & d z \\ 0.0 & -8.15 & -33.1\end{array}\right) \quad$ \$Can 1

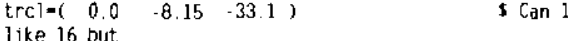

trci=( - $7.015-20.25-33.1) \quad \$$ Can 2

$\begin{array}{llll}\text { like } 16 \text { but } & \\ \operatorname{trc} l=(7.015 & -20.25-33.1) & 5 \text { Can } 3\end{array}$

Upper Can $(0,0,0)$. Universe 5

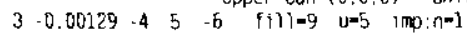

$\operatorname{trcl}=(-2) \quad d y \quad d z$

trcl=( $7.0581 \quad 4.075 \quad-33.1) \quad$ s Can 1

like 19 but

trcl-(14.0295 $16.200-33.1) \quad$ S Can 2

trcl=\{21.0445 $4.0498 \cdot 33.1) \quad \$$ Can 3

$3.0 .0029-4$ Upper Can $(1,0.0)$ - Universe 6

$3-0.00129-4 \quad 5$-6 filt:- $u=6$ imp: $n=1$

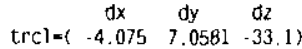

like 22 but

$\operatorname{trc})=\left(\begin{array}{llll}-21.0445 & 4.0498 & -33 & 1\end{array}\right)$

Can 1

like 22 but

trct- $(-14.029516 .200-33.1)$

s Can 3

$3 \cdot 0.00129-45-6$ fin Can $(0.1 .0)$ - Universe 7

$\begin{array}{llllll}-4 & 5 & -6 & \text { fill-9 } & u=7 & \text { imp: } n-1\end{array}$

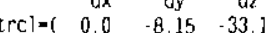

3 Can

like 25 but

trcl-( $-7.015 \cdot 20.25-33.1)$ s Can 2

trel=( $7.015-20.25-33.1) \quad$ s Can 3 
HNF-6179 Rev. 0

$\begin{array}{lll}\text { C } & \text { Drum - Universe } 2\end{array}$

$4.0 .92 \quad-78 \cdot 9 \# 10 \# 11$ \#12 imo:n-1 $u=2$ s Interior

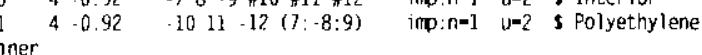

$32 \quad 2-3.92-1314-15(10 \cdot-11 \cdot 12)$ imp:n=1 u=2 s Drum

$33 \quad 3-0.0012913:-14: 15$ imp:n+1 $u=2$ s Air around Drum

Orum - Universe 3

$34 \quad 4-0.92 \quad-78 \quad 9 \quad \# 13 \quad \# 14 \quad \# 15 \quad$ imp: $n=1 \quad u=3$ s Interior

$35 \quad 4-0.92 \quad-1011 \cdot 12(7:-8: 9) \quad$ imp:n-1 $\quad u=3$ s Polyethylene

$36 \quad 2-3.92 \quad-1314 \cdot 15(10:-11: 12)$

$37-0.00129,13:-14: 15$

imo: $n-1 \quad u=3$ s Drum

imp: $n=1 \quad u=3$ s Air around Drum

Orum - Untverse 4

- $-0.92 \quad-78-9 \# 16 \# 17 \# 18$ imp:n=1 $u=4$ s Interior

$4-0.92 \quad-1011-12(7:-8: 9) \quad$ imp:n=1 $u=4$ s Polyethylene

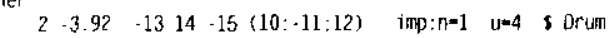

$41 \quad 3-0.0012913:-14: 15 \quad$ imp:n-1 u=4 s Air around Drumc

c $\quad$ Drum. Universe 5

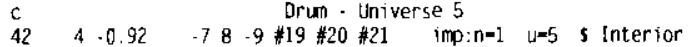

$43 \quad 4-0.92 \quad-1011-12(7:-8: 9) \quad$ imp:n=1 $\quad u=5$ s Polyethylene

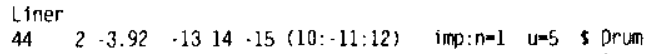

$45 \quad 3 \cdot 0.0012913:-14: 15 \quad$ imp:n=1 $u=5$ s Air around Drum

c $\quad$ Drum - Universe 6

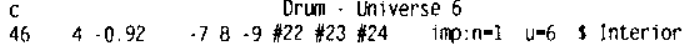

$47 \quad 4-0.92 \quad-1011-12(7:-8: 9)$ imp:n=1 $u=6$, Polyethylene

$\begin{array}{llllll}\text { Liner } & 2-3.92 & -1314 \cdot 15(10:-11: 12) \quad \text { imp: } n=1 \quad u=6 & \text { s Drum }\end{array}$

$49 \quad 3 \cdot 0.0012913:-14: 15$ imp:n=1 $u=6$ s Air around Drum

$$
\text { C Drum - Universe } 7
$$

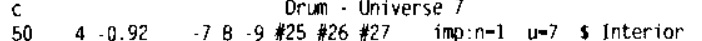

$51 \quad 4 \quad-0.92 \quad-1011-12(7:-8: 9) \quad$ imp:n-1 $\quad \mathrm{u}=7$ s Polyethylene

Liner
52

$53 \quad 3 \cdot 0.0012913:-14: 15$ imp:n=1 $u=7$ s Air around Drunc

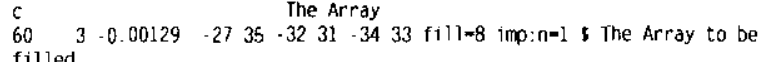

c Hexagonal Universe - Universe 8

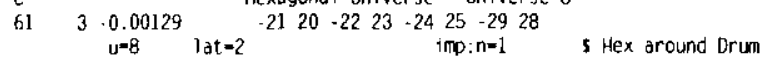
$u=8 \quad l a t=2$
$f(11)=-15: 15 \quad-15: 15 \quad-1: 2$

$z-1$

$x=\begin{array}{lllllllllllllll}-14 & -12 & -10 & -8 & -6 & -4 & -2 & 0 & 2 & 4 & 6 & 8 & 10 & 12 & 14\end{array}$ 8888888888888888888888888888888

$y=-15$

$y=14$

$y=-13$

$y=-12$

$y=-11$

$y=-10$

$y=-9$

$y=-9$

$y=-8$

$y=-7$

$y=-6$

$y=-5$

$y=-5$

$y=-4$

$y=-3$

$y=-2$

$y=1$

$y=0$

$y=1$ $y=2$

$y-3$

$y=4$

$y=5$

$y=6$

$y=7$

$y=8$

$y-9$

$y=10$

$y=11$

$y=12$

$y=13$

$y=14$

$y=15$

c

$c$
$c$
$c$

$y=-15$

$y=-14$

$y=-13$

$y=-12$

$y=-11$

$y=-10$

$y=-9$

$y=-8$

$y=-7$

$y=-6$

$y=-5$

$y=-4$

$y=-3$

$y=-2$

$y=1$

$y=0$

$y=1$

$y=2$

$y=3$

$y=4$

$y=5$

$y-6$

$y=7$

$y-8$

$y=9$

$y=10$

$y-11$

$y=12$

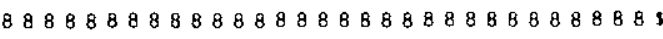
8 $8888888888888 B 88888888888886885$ 8888888888888 B B888888B8888 8888 B 8888888888B88888888B8888888888

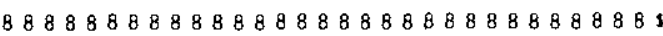
$888888888888888888888888888888 B$ 88888888888888888888888888868885 8688888888888888888868888888888 888888888888888888888888888888 8 8888888888888888888888888888888 । 88888888888888888888888888868885 8 8 B 88888888888888888888888B88885 $8888888888888888888888888888888 \mathrm{~s}$ $88888 B 88888888888888888888888885$

$z=0$

$\begin{array}{lllllllllllllll}-14 & -12 & -10 & -8 & -6 & -4 & -2 & 0 & 2 & 4 & 6 & 8 & 10 & 12 & 14\end{array}$

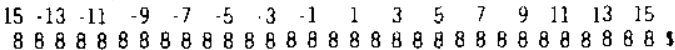
B8888B8888888888888888B888B88885

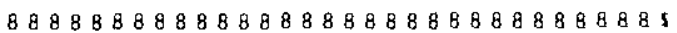
$88 B 88888888888888888 B 88888888885$

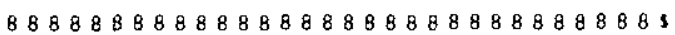
B 8888688888823423888888888868885 88 $888888 B 88$ 84234288B888888688885 88888888888234234234234234888883 8888888888342342342342342388888 s 8888888888234234234234234288888 s 88888888884234234234234234888885 8888888888342342342342342388888 s

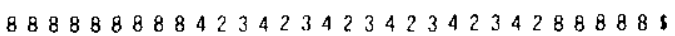
88888888834234234234234234888885 $888888 B 8823423453423428$ 888888B8s 88888688842342342342348888888885 $888888342342342342342388888 B 8885$ 88888823423423423423428868888885 88888842342342342342388888888883 $88888834234234234234288888 B 88885$ 88888823423423423423488888888885 88888842342342342342388888888885 8888883423423423423428888888888 s 8888882342342342342348888888888 s B 88888423423423288B8888888888885 B8888B3423423423888888888888888s $8888882342342342888888 B 88888688$ s 8888888884234234888888888868888 s 
$y=13$

$y=14$

$y=15$

$c$

c

$y=-13$

$y=-12$

$y=11$

$y=10$

$y=-9$

$y=-8$

$y=-7$

$y=-6$

$y=-5$

$y=-4$

$y=-3$

$y *-2$

$y=1$

$y=0$

$y=1$

$y=2$

$y=3$

$y=4$

$y=5$

$y * 6$

$y=7$

$y=8$

$y=9$

$y=10$

$y-11$

$y=12$

$y=13$

$y=14$

$y=15$

$c$

$c$
$c$
$c$

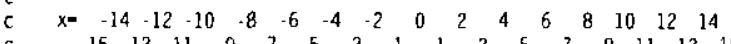
$\begin{array}{lllllllllllllllllllll}\cdot 15 & -13 & -11 & -9 & -7 & -5 & -3 & -1 & 1 & 3 & 5 & 7 & 9 & 11 & 13 & 15\end{array}$ $y *-15$

$y=-14$

$y=-13$

$y=12$

$y=-11$

$y=-10$

8888888883423423888888888888888 s $8888888882342342888888 B 88888888$ s 88888888842342348888888888888885

$z=1$

$\begin{array}{lllllllllllllll}-14 & -12 & -10 & -8 & -6 & -4 & -2 & 0 & 2 & 4 & 6 & 8 & 10 & 12 & 14\end{array}$

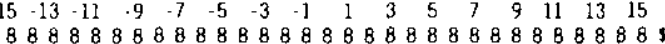

88888888888888888888888888888885

88888888888888888888888888888885

$8 B 8888 B 88888888 B 888 B 88888888888 \mathrm{~s}$

8888888888888888888888888888888 s

88888888888888888888888888888885

88 8888888B88888B8888B88888888B85

$8888888888888888888888888888888 \mathrm{~s}$

$B 8 B 8888 B B 88 B B 888 B B B B B 88 B 8 B 888 B 85$

8 $88888888888888888888888888888 B 5$

8 $8888888888888888888888888888 B 8$ s

$888888888888888888888888 B 8888885$

$8 B 888888888888888888888888888885$

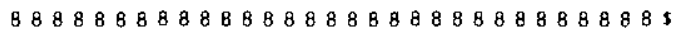

8888688888 B888888888888888888885

88 8888888B8888888888888888888B85

$888 B 8888 B 8888888888888888888888 \mathrm{~S}$

$8 B 888888888888888888888888888$ g 5 s

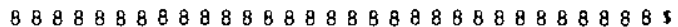

$8888 B 88888 B 88888888888888888888$ s

$8888888888888888888888888888888 \mathrm{~s}$

$8888888888888 B 888 B 8888 B 888888885$

88888888888888888888888888888885

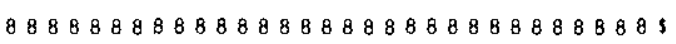

$8888888 B 88888888888888888888888 \mathrm{~s}$

88888888888888888888888888888885

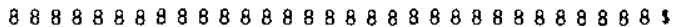

$888888888888888888 B 88888888888$ क

$888888 B 888888 B 888888888888 B B 88 B \mathrm{~S}$

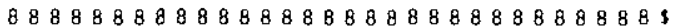

88888888888888888888888888888885

$8888888 B 8888$ 88B8888888888888B88

8888888888888888888888888888888 s

$8888 B 8888888888888888888 B 888888$ s

$88888 B 88888888888$ B B 8888B88888885

$888888888888888888 B 8888888888885$

$8888 B 8 B 8888 B B 8888 B 888888888 B 888 \mathrm{~s}$ $y=-9$

$y=-8$

$y=-7$

$y=-6$

$y=-5$

$y=-4$

$y=-3$

$y=-2$

$y=-1$

$y=0$

$y=1$

$y=2$

$y=3$

$y=4$

$y=5$

$y=6$

$y=7$

$y=8$

$y=9$

$y=10$

$y=11$

$y=12$

$y=13$

$y=14$

$y=15$

c

$c$

$$
\begin{aligned}
& c \\
& 70
\end{aligned}
$$

Surface Definitions

$\begin{array}{llllllll}3 \cdot 0.00129 & -32 & 31 & -34 & 33 & -36 & 27 & \text { imo: } n=1\end{array}$

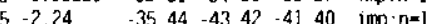

$\begin{array}{llllllll}-2.24 & -45 & 36 & .43 & 42 & -41 & 40 & \text { imp: } n=1\end{array}$

$\begin{array}{lllllllll}5 & -2.24 & -36 & 35 & -43 & 42 & -41 & 32 & \text { imp: } n=1\end{array}$

$\begin{array}{lllllllll}5 & -2.24 & -36 & 35 & -43 & 42 & .31 & 40 & \text { img }: n=1\end{array}$

$\begin{array}{lllllllll}5 & -2.24 & -36 & 35 & -33 & 42 & -32 & 31 & \text { imp: } n=1\end{array}$

.2 .24

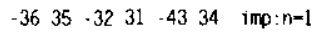

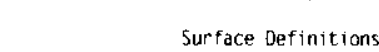

Billets

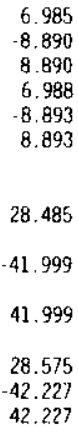

Bi11

D2

3

$5 \quad p z$

6

$7 \quad c$

Interior

$8 \quad$ pz

Interior

$9 \quad \mathrm{pz}$

$10 \mathrm{C2}$

$11 \quad \mathrm{Dz}$

is $c 2$

Drum
8888888888888888 8888888888B8888s

8888888888888888 s

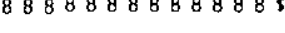

88888888888 s

$88888888 B 88$ s

88888888888888888888888888888885

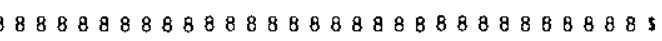

8888888888888888s

(8) $8888888 B 8888 B 8 B 8 B 88 B 8 B 5$

$88 B 888888888888888888 B 888888888$ s

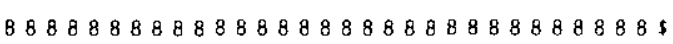

$8888888 B 88888 B 8888868888888$ s

888888888888888 s

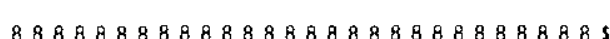

8886888888888888888688888888888 s

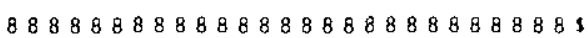

$888888888888868888 B 8888868$ s

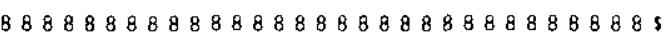

The Rest of the Room

Air above array

Concrete floor

s Concrete Celing

s Right Concrete

3 Left Concrete

I Front concrete

s Back Concrete

s The Great void

- Radius of waste 8ottom of Wast - Top of Waste

5 Outside of Can

- Bottan of Can

s Top of can

5 Radius of

s Bottom of

s Top of Interior

5 Radius of liner

5 Bottom of Liner

$\checkmark$ Top of Liner

$\checkmark$ Radius of Orian 
HNF-6179 Rev. 0

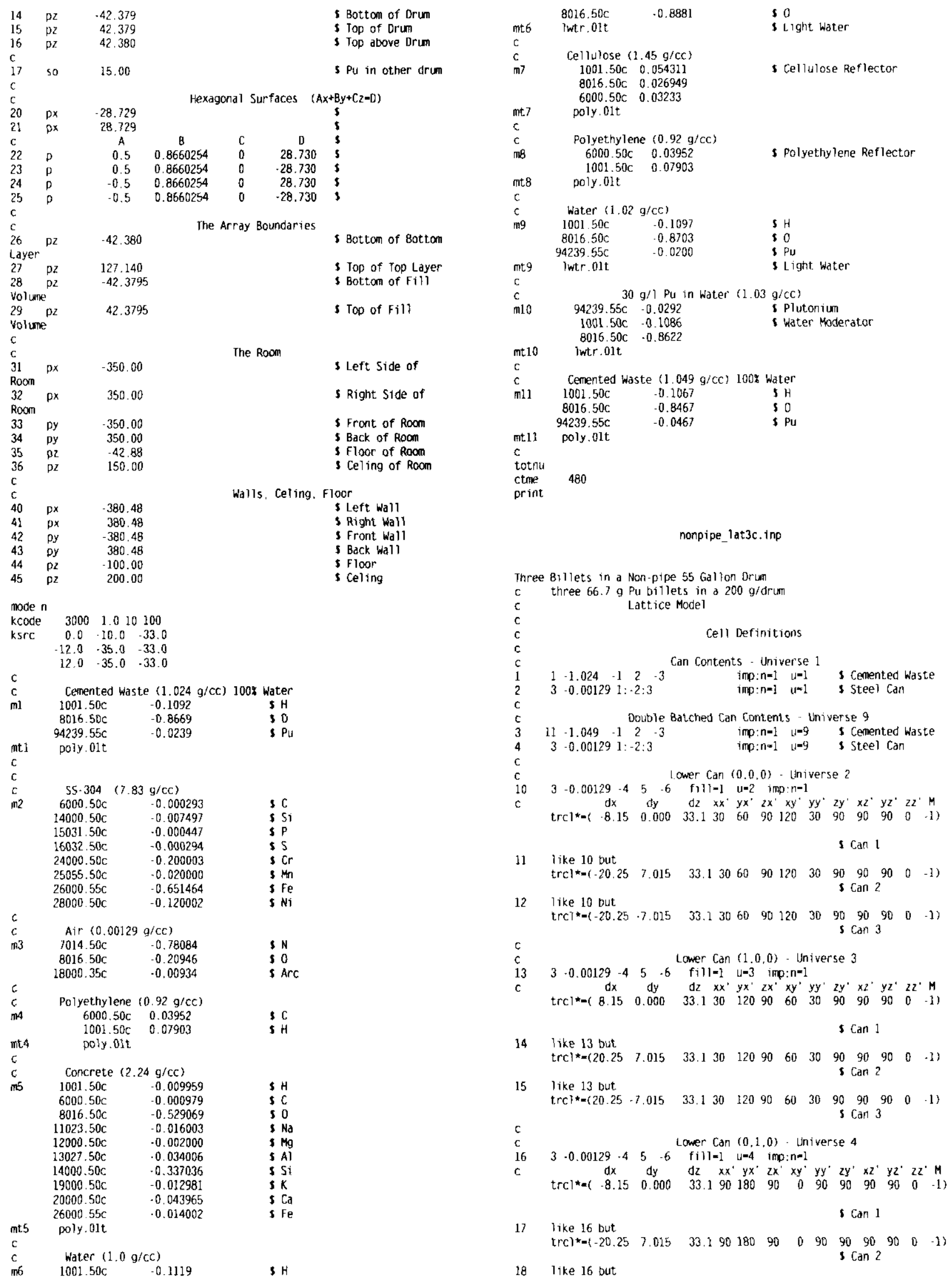




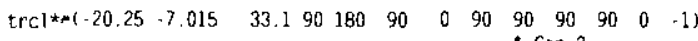
s Can 3

$c$

$193-0.00129-4 \quad 5 \quad-6$ Upper Can $(0.0 .0)$ - Universe 5

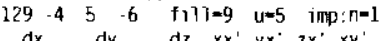

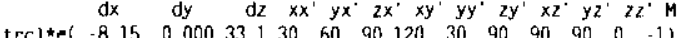

$20 \quad$ like 19 but

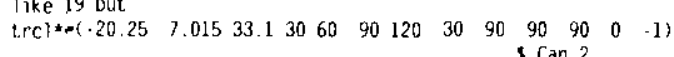

21 like 19 but

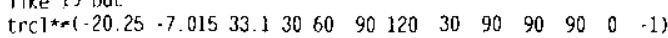
5 Can 3

c $\quad$ Upper Can $(1.0,0)$ - Universe 6

$22 \quad 3 \cdot 0.00129-4 \quad 5 \quad-6$ fillime $u=6$ imp: $n=I$

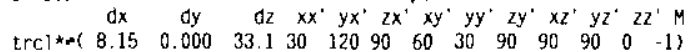
$\left.\begin{array}{lllllllllllll}\text { trcl })^{*}(20.25 & 7.015 & 33.1 & 30 & 120 & 90 & 60 & 30 & 90 & 90 & 90 & 0 & -1\end{array}\right)$ 24 like 22 but 5 Can 2

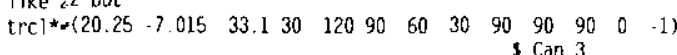

Upper Can $(0,1,0)$. Universe 7

$25 \quad 3-0.00129-4 \quad 5 \quad-6 \quad$ fi) $1-9 \quad u=7$ imp: $n=1$

c $d x \quad d y \quad d z \quad x x^{\prime} y x^{\prime} z x^{\prime} x y^{\circ} y y^{\prime} z y^{\prime} x z^{\prime} y z^{\prime} z z^{\prime} M$

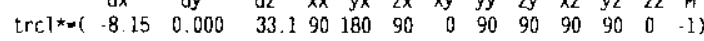

26 like 25 but

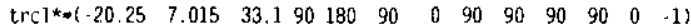

like 25 but

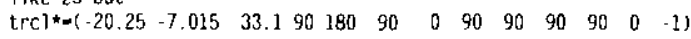
s $\operatorname{can} 3$

C. Orum - Universe 2

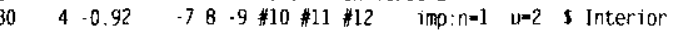

$31 \quad 4-0.92 \quad-1011-12(7:-8: 9) \quad i m p: n=1 \quad u=2$ s Polyethylene

Liner

$32 \quad 2 \cdot 3.92-1314-15(10:-11: 12) \quad$ ino:n=1 $u=2$ s Drum

$33 \quad 3-0.0012913:-14: 15 \quad$ imp:n=1 $u=2$ s Air around Drum

c Drum - Universe 3

$34 \quad 4 \quad-0.92 \quad .78-9 \quad \# 13 \quad \# 14 \quad \# 15 \quad$ imp:n=1 $u=3$ s Interior

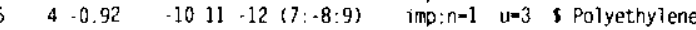

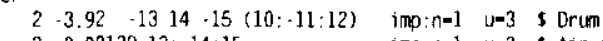

$3-0.0012913:-14: 15$

imp:n-1 $u=3$ s Air around Orum

Drum - Universe 4

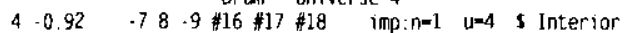

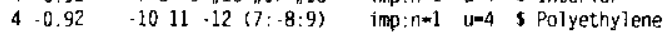

$2 \cdot 3.92-1314-15(10:-11: 12)$

$3 \cdot 0.0012913:-14: 15$

imo: $n-1 \quad u=4$ s Drum

ing: $n-1 \quad u=4$, Air around Orumc

Drum - Universe 5

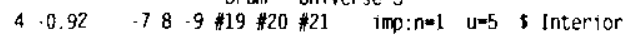

$\begin{array}{lllll}4 & -0.92-1011-12(7:-8: 9) \quad \text { imp: } n=1 \quad u=5 & \text { s Polyethylene }\end{array}$

$2-3.92-1314-15(10:-11: 12) \quad$ imp:n=1 $u=5$ s Orum

3 $-0.0012913:-14: 15$ imp:n-1 $u=5$ s Air around Drum

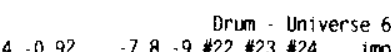

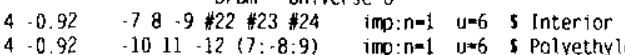

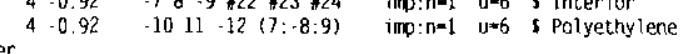

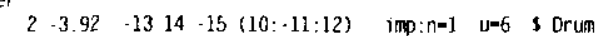

$3-0.0012913:-14: 15$ imp:n=1 $u=6$ s Air around Drum

Drum - Universe 7
$4-0.92 \quad 78-9 \quad \# 25 \# 26 \# 27$ imp:n-1 $\quad \mathrm{w}=7$ s Interior

4. $0.92 \quad-1011 \cdot 12(7:-8: 9) \quad$ imp:n=1 $u=7$ s Polyethylene

liner

$52 \quad 2 \cdot 3.92 \quad .13 \quad 14 \cdot-15(10:-11: 12)$ imp:n-1 u-7 s Orum

$53 \quad 3 \cdot 0.0012913:-14: 15$ imp: $n=1 \quad u=7$, Air around Drumc $\begin{array}{lllll}\text { C The Array } \\ 60 \quad 3 \cdot 0.00129 & -2735-3231-3433 \text { fill } \approx 8 \text { imp:n=1 s The Array to be } \\ \text { filled } & \end{array}$

c Hexagonal Universe - Universe 8

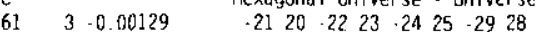

u-8 lat $=2$ imp:n-1 S Hex around Drum

$2=-1$

$\begin{array}{lllllllllllllll}-14 & -12 & -10 & -8 & -6 & -4 & -2 & 0 & 2 & 4 & 6 & 8 & 10 & 12 & 14\end{array}$ $\begin{array}{llllllllllllllll}-15 & -13 & 11 & -9 & -7 & -5 & -3 & -1 & 1 & 3 & 5 & 7 & 9 & 11 & 13 & 15\end{array}$ 8888888888888888888888 B B8888888s

$y=-15$

$y=-14$

$y=-13$

$y=-12$

$y=-11$

$y=-10$

$y=-9$

$y=-8$

$y=-7$

$y=-6$

$y=-5$

$y=-4$

$y=-3$

$y=-2$

$y=-1$

$y=0$

$y=1$

$y=2$

$y=3$

$y=4$

$y=5$

$y=6$

$y=7$

$y=8$

$y-9$

$y=10$

$y=11$

$y=12$

$y=13$

$y=14$

$y=15$

$c$

c

$c$

$y=-15$

$y=-14$

$y=-13$

$y=-12$

$y=-11$

$y=-10$

$888888888888888888888 B 8888888885$

8888888888888888888888888888888 s

$888888888888888888888888888888 \mathrm{~B}$ s

$8888888888888888888888 B 88888 B 8$ s

8 8888 8 8888888888888888888888888 s

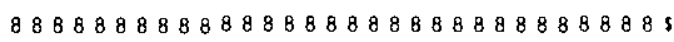

8888888888888888888888888888888 s

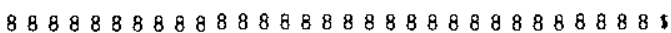

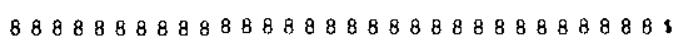

8888888888888888888888888888888 s

8 8888888888888888888888888888885

8888888888888888888888888888888 s

$8 B 88888888888888888888888888888$ s

$8888 B 8888$ B B 8888888888B8888888885

8888888888888888888888888888888 s

8888888888888888888888888868888 s

$8 B 8888888888 B 8888 B 8888888888888$ s

88888888888888888888888888888885

$8888 B 888888888688888$ B 88888B88885

$888888888888888888888888888888 B \mathrm{~s}$

$88 B 8888888888888888888888888888$ s

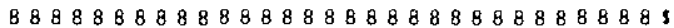

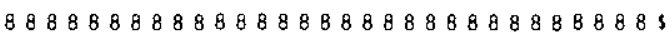

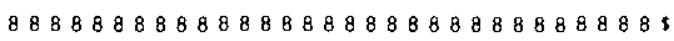

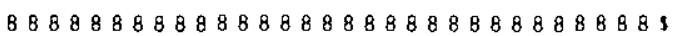

88888888888888888888888888888885

8888888868888888888688888 B888885

$8 B 88888 B 8888888888 B 88888888888 B \mathrm{~s}$

888888888888888868888888888888 8

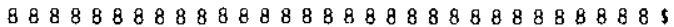

$z=0$

$=\begin{array}{lllllllllllllll}-14 & -12 & -10 & -8 & -6 & -4 & -2 & 0 & 2 & 4 & 6 & 8 & 10 & 12 & 14\end{array}$ $\begin{array}{llllllllllllllll}15 & -13 & -11 & -9 & -7 & .5 & -3 & -1 & 1 & 3 & 5 & 7 & 9 & 11 & 13 & 15\end{array}$ 88868888888888888888688888 B 8888 s $8888888888888 B 8888 B 888888888886$ s 8888888888888888888888888889888 s $888888888 B 8888888888886888888 B 85$ 888 8 8B88888888888888888888888885

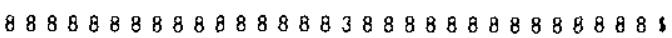
8888888888888888888 B88888B8888 B 
HNF-6179 Rev. 0

$y=1$

$y=2$

$y=3$

$y=4$

$y * 5$

$y=6$

$y=7$

$y=8$

$y=9$

$y=10$

$y=11$

$y=12$

$y=13$

$y=14$

$y=15$

$\mathrm{c}$

c

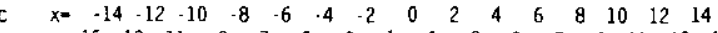

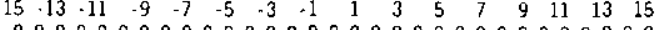

$y=-1$

$y=0$

$y=1$

$y=2$

$8888 B 8888888888888 B 8888888888885$

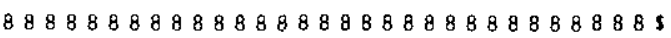
$888888888 B B 88888888888888888888$ s

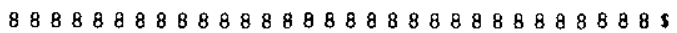
88888888888888888888886888888885

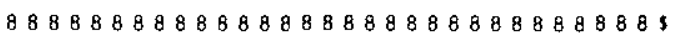
$8 B 8 B 88888 B 8886888888 B 8888$ B B B B885 8 B 888886888888853888888888888885 88888888888889848888886888888885 8888888888888888888888888888888 s 8888888888888888888888888888888 s 88888888888888888888888688888885

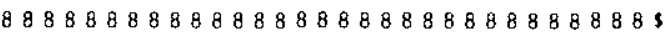
$888888888888 B 888888888898888888$ s

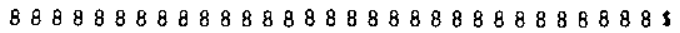
888888888888888888888888888888B5 88888888 B 88888888888B8888888B88s 868888888888888888888888888888 \& B 88 \& $8 B 88888 B 888888888 B 88888888$ B 8 8 886888888888888888888888888 B 85

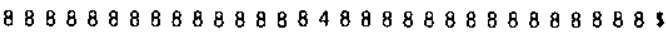
$88888 B 88888 B 8888886888$ B88886888s 8888888888888888888888888888888 s 8888888 8 88888888888888 8 8 B8888B8

\section{$2-1$}

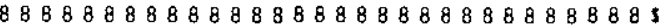
$88889888888888888 B 8888888888888$ s $88888888888888888888 B 88888888 B 8$ s 8 888888888888888888888888888888 s 8888888688888888888888888888 8 8 8888888888888888888888888888888 s 888888888888888888888888888868 s 8888888888888888888888888888886 s 888B888888888888888888888B88888

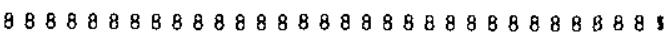
888888888888888888888888888888 s 8888888888888888888888888888888 s 888888B888889888888888888888888 8888888888888888888888888888888 s $8 B 88888888888888888888888888888$ s 88888888888888888888898888888885 $8888 B 888888888886888888888 B 888 B$ s

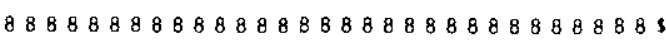

$y=3$

$y=4$

$y=5$

$y=6$

$y=7$

$y=8$

$y=9$

$y=10$

$y=11$

$y=12$

$y=13$

$y=14$

$y=15$

c$$
\text { c }
$$

$y=-12$

$y=-11$

$y=-10$

$y * \cdot 9$

$y=-8$

$y=-7$

$y=-6$

$y=-5$

$y=-4$

$y=-3$

$y=-2$

$y=-1$

$y=0$

$y=1$

$y=2$

$y=3$

$y=4$

$y=5$

$y=6$

$y=7$

$y=8$

$y=9$

$y=10$

$y=11$

$y=12$

$y=13$
$8 B 8888 B 888888 B 88888888888888 B 885$

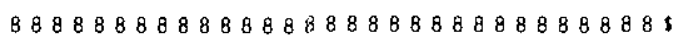
$8888 B 8888 B 8888 B 8 B 888888688888 B 8$ s $88 B 888 B 88888 B 8 B 88888888888888$ \&

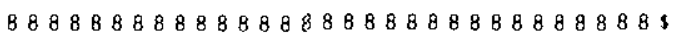
$8888 B 888 B 888888888888 B 888888 B 885$ B888888888888888888888888888888s $8888888888888888888888888888888 \mathrm{~s}$ $88888 B 88888888888 B 88888 B 888888 B$ s $88888888888888888 B 8888 B 88888888 \mathrm{~s}$ 8888888888888868888888888888888 s 88888888888888 \&888B888888888888s

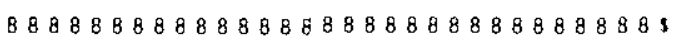

\section{$2=2$}

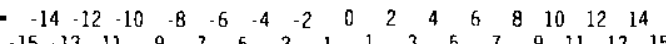
$8 B 888 B 8 B 888 B 88888888888 B 886888 B$ s

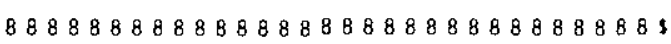
$888 B 88888 B 8888888888 B 888888$ B888 $8 B 8888888888888888888888 B 88888 B$ s 8888888888888888888888888888888 s $88 B 888888 B 8888 B 8888888888 B 88888$ s 8888888888886888888888888888888 s $8888888888888888888888888888 B 88 \mathrm{~s}$ 8 8 $8 B 88888 B 888 B 888888888888888885$ $8 B 88888888888888888888888888888$ s

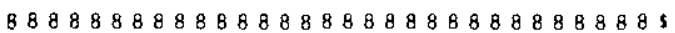
$888 B 88888 B 888888888888888 B 88888$ s

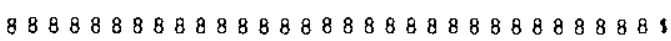
$8888888888 B 8888 B 88888888888 B 888$ s 8888888888888888888868888888888 s $88 B 8888888888 B 8888868888 B 888888$ s B 888888888888888888888888888888 s 88 $88888888888888888888888888888 \mathrm{~s}$ 8888888688888888888888888888888 s 8888888888888888888888888888888 s

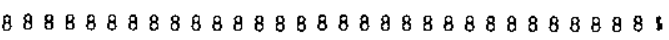
8 $88888888888888888888888888888 B$ s

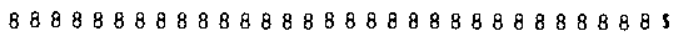

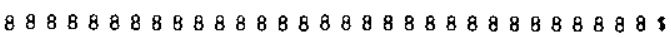
8888888888888888888888888888886 s

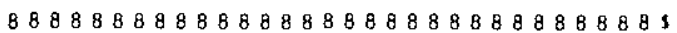
$888 B 88888 B 8888 B 8 B 8888 B B 8888888$ s, $88888888888888888888688888 B 8888$ s $888888688888888888 B 888888888888$ s 
HNF-6179 Rev. 0

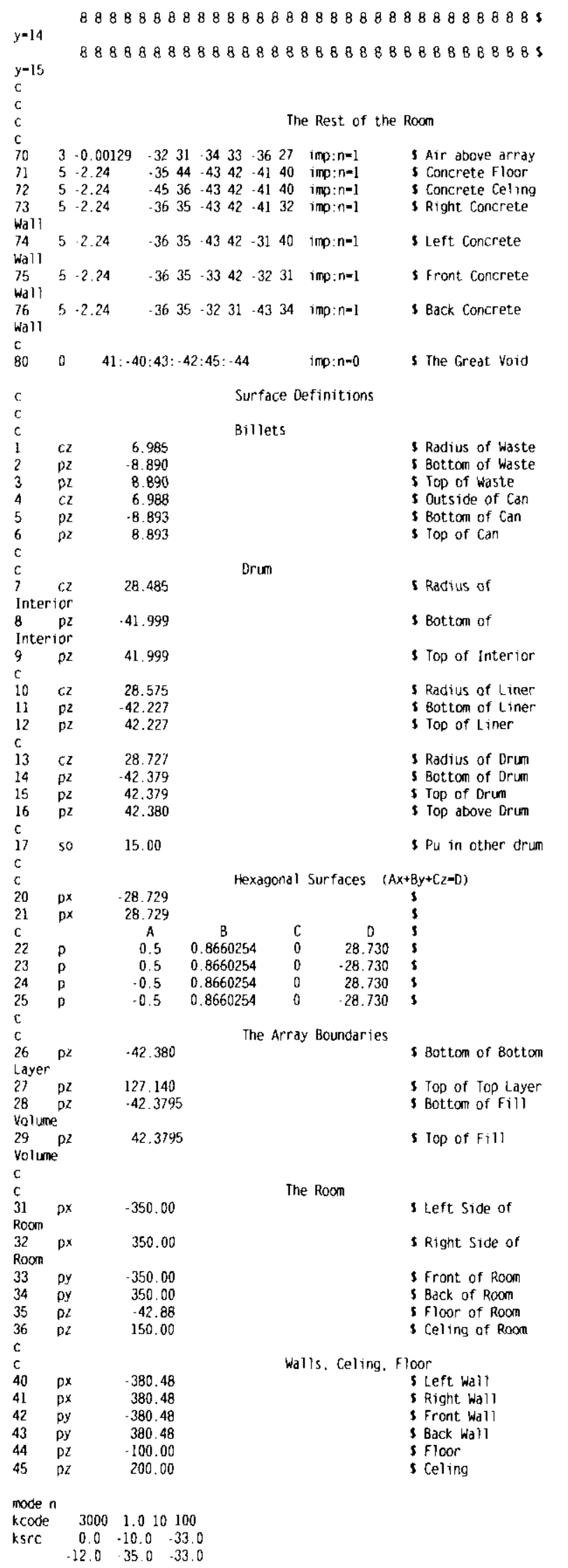

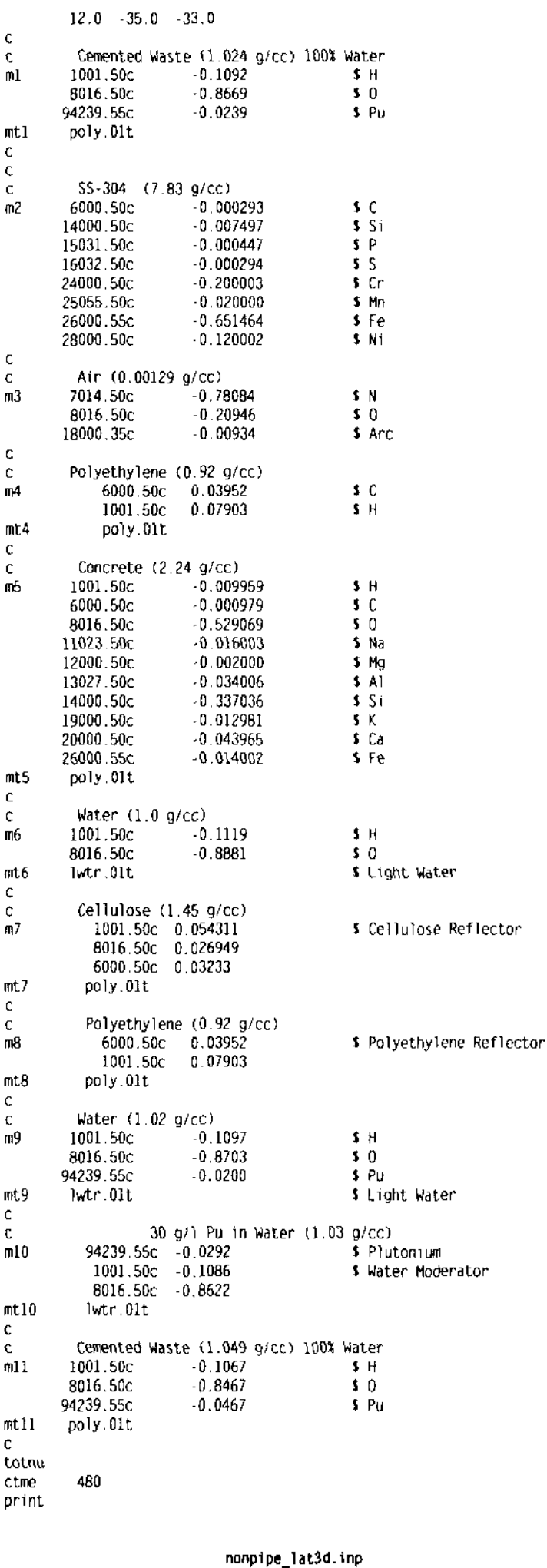

Three Billets in a Non-pipe 55 Gallon Drum three $66.7 \mathrm{~g}$ Pu billets in a $200 \mathrm{~g} / \mathrm{drum}$ Lattice Model

Cell Defintions

Can Contents - Universe 1 


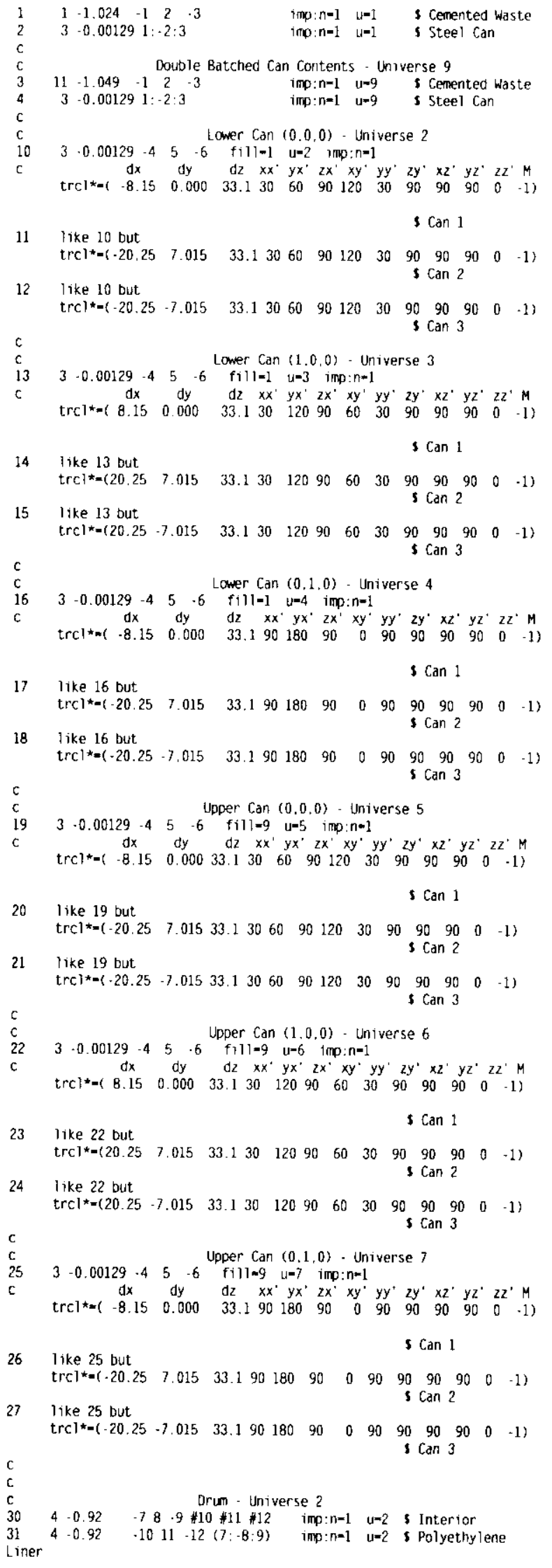

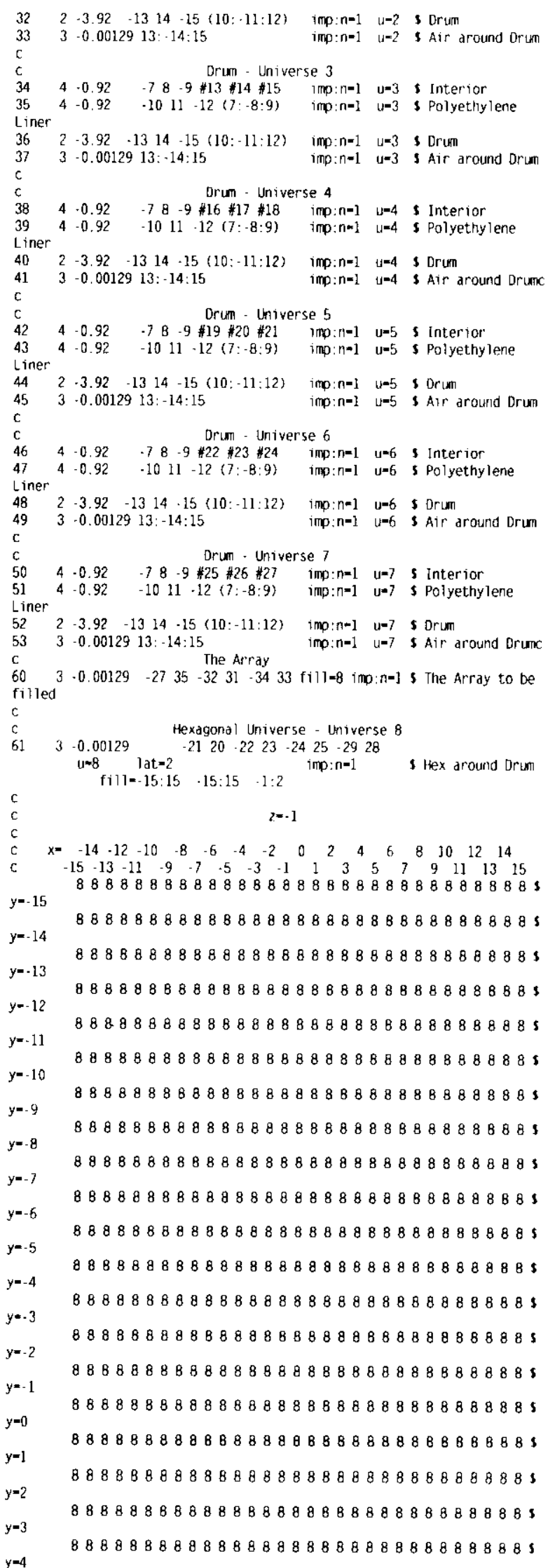


HNF-6179 Rev. 0

8888888888888888888888888888888 s 8 888888888688888888888888886888 s 8 $88888888 B 8888888888888888888$ B8s 88888888888888888888688888886885

$888898 B 888888 B 8888 B 8888 B 88888885$ $88888888888888888 B 8888888868888$ s 88 $8868888 B 8888888888 B 888888888$ \& $8 B 88888888888888888888888888888$ $888888888888888888 B 8888 B 88 B 888 B$ $8888888888888888 B 88888888888888$ s $888 B 888888888888888888888888888$ 88888888888888888888888888888885

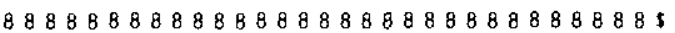
8 888888888888888889888888888888 s $8888888888888888888888886888888 \mathrm{~s}$ 88888868888886888888888888888885 88888888888823423888888888888885

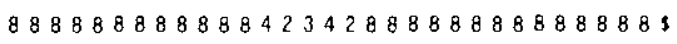
8888888688823423423423423488888 s 88888888883423423423423423888885 8888688888234234234234234288888 s 8888888888423423423423423488888 s 88888888883423423423423423888885 88888888842342342342342342888889 8888888863423423423423423488888 s B B888888823423453423428888688883 8888888884234234234234888888888 s 88888834234234234234238888888885 B8888823423423423423428888888885 8888884234234234234238888888888 s 88888834234234234234288888888885 88888823423423423423488888888885 88888842342342342342388888888883 88888334234234234234288888888885 88888823423423423423488888 B8888s 8888884234234232888888888888888 s 88888834234234238888888888888885 88888823423423428888888888888885 88B8888884234234888888888888B88s 88888888834234238888888888888885 88888888823423428888888888888885 88 8B888884234234888888888B8888Bs

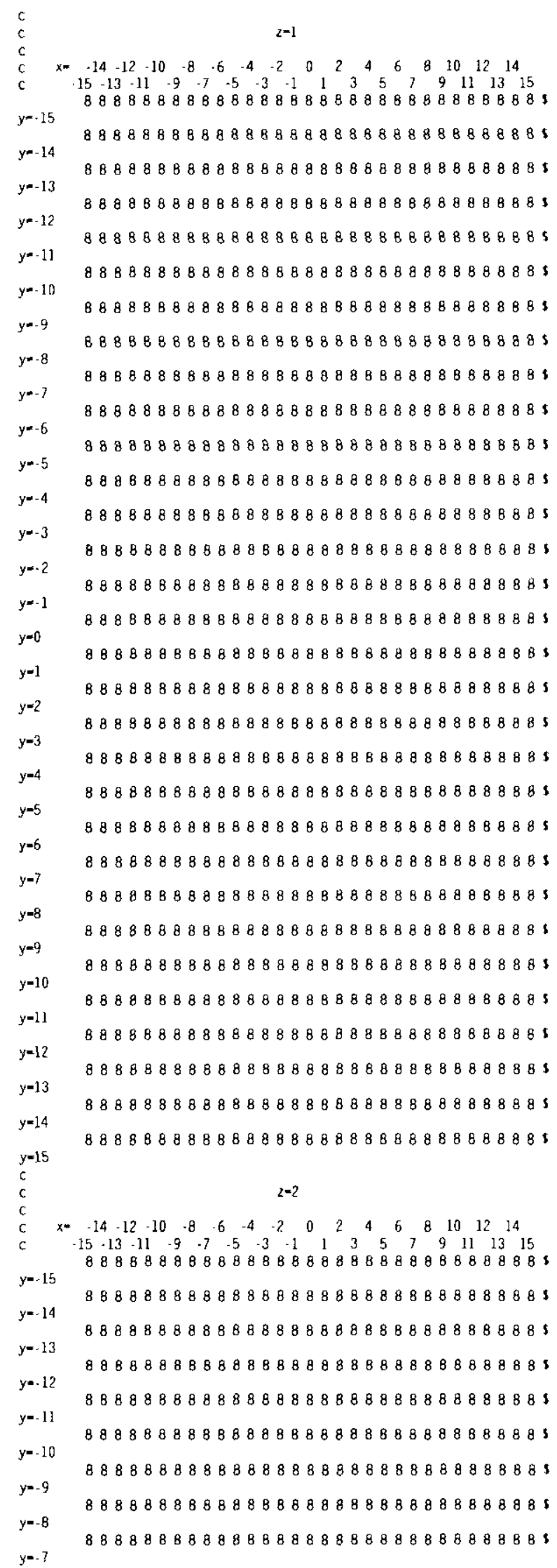


HNF-6179 Rev. 0

8888888888888888888888888888888 s $y=\cdot 6$

$y=-5$

$y=-4$

$y=-3$

$y=-2$

$y=-1$

$y=0$

$y-1$

$y=2$

$y=3$

$y-4$

$y=5$

$y-6$

$y=7$

$y=8$

$y=9$

$y=10$

$y=11$

$y=12$

$y=13$

$y=14$

$y=15$

888888888888888888888 B8888888s

$8888888888888888 B 888888888888$ B

888B8888888888888888888888888885

8888888888888888888888888888888 s

$8888 B 88888888888888888 B 88888888$ s

$88 B 888888888888888888888888888$ s

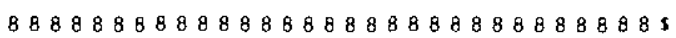

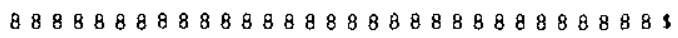

$88888888888888888888888888888 B 8$ s

$888888888888 B 888886888888888888$ s

$8988888888888888888888888888888 \mathrm{~s}$

8888888888888888888888888889868 s

8888888888888B888888888888888885

88888888888888888888888888888885

$888 B 888888888888 B 88888888 B 888 B 8$ s

88888888868888888888888888888885

88888988888888888898888888888885

$8888 B 88888888888 B 8888888888888 B$ s

888888888888888888888888888888 s s

88888888888888888868888888868885

The Rest of the Room

$\begin{array}{lllllllllll}70 & 3 & -0.00129 & -32 & 31 & -34 & 33 & -36 & 27 & \text { imp: } n=1\end{array}$

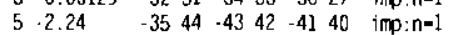

$\begin{array}{lllllllll}5 & -2.24 & -45 & 36 & -43 & 42 & -41 & 40 & \mathrm{imp}: n=1\end{array}$

$\begin{array}{lllllllll}5 & -2.24 & -36 & 35 & -43 & 42 & -41 & 32 & \text { imp: } n=1\end{array}$

$\begin{array}{llllllllll}74 & 5 & -2.24 & -36 & 35 & -43 & 42 & -31 & 40 & \text { imo:n-1 }\end{array}$

$\begin{array}{llllllllll}74 & 5 & -2.24 & -36 & 35 & -43 & 42 & -31 & 40 & \text { imp:n=1 } \\ \text { Wa11 } & & & & & & & & & \\ 75 & 5 & -2.24 & -36 & 35 & -33 & 42 & -32 & 31 & \text { imp:n=1 }\end{array}$

wâll

$\begin{array}{lllllllllll}76 & 5 & -2.24 & -36 & 35 & -32 & 31 & -43 & 34 & i m p: n=1\end{array}$ wall

$\begin{aligned} & \text { c } \\ & 80\end{aligned} 0 \quad 41:-40: 43:-42: 45:-44 \quad$ imp: $n=0$

- Air above array s Concrete Floor

5 Concrete Celing

right Concrete

Left Concrete

$s$ Front Concrete

$\checkmark$ Back Concrete

$\begin{array}{lr}\mathrm{CZ} & 6.985 \\ \mathrm{PZ} & -8.890\end{array}$

$02-\quad-8.890$

CZ $\quad 6.988$

p2 $\quad-8.893$

p2

interior

r

8.85

28.485

.41 .999

41.999

$9 \quad \mathrm{pz}$

$\begin{array}{lll}0 & \mathrm{CZ} & 28.575\end{array}$

$\begin{array}{llr}11 & \text { p2 } & -42.227 \\ 12 & \text { pz } & 42.227\end{array}$

$\begin{array}{lll}13 & \mathrm{Cl} & 28.727\end{array}$

$\begin{array}{rrr}13 & \mathrm{CZ} & 28.727 \\ 14 & \mathrm{pz} & -42.379\end{array}$

$\begin{array}{lll}15 & \mathrm{pz} & 42.379 \\ 16 & \mathrm{pz} & 42.380\end{array}$

$\begin{array}{lll}\text { c } & \text { so } & 15.00\end{array}$

17 so $\quad 15.00$
Surface Definitions

Billets

s The Great void
5 Radius of Waste Bottom of Waste

5 Top of Waste

s Outside of can $s$ Botton of Can

s Top of Can

Drum

1 Radius of

5 Bottom of

s Top of Interior

$\$$ Radius of Liner $s$ Bottom of Liner

s Top of Liner

S Radius of Drum

5 Bottom of Drum

s Top of Drum

1 Top above Drum

$5 \mathrm{Pu}$ in other drum

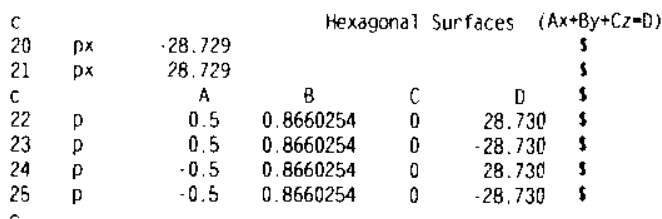

$26 \mathrm{pz} \quad-42.380 \quad$ The Array Boundaries

$27 \mathrm{pz} \quad 127.140 \quad$ STop of Top Layer

$28 \mathrm{pz} \quad-42.3795 \quad$ S Botton of Fill

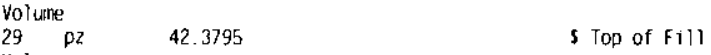

volunie

$31 \rho x-350.00$

$\rho x \quad 350.00$

py $\quad-350.00$

py $\quad 350.00$

$\begin{array}{ll}\mathrm{pZ} & -42,88 \\ \mathrm{pZ} & 150.00\end{array}$

The Room

\$ Left Side of

5 Right side of

5 Front of Room

5 Back of Room

5 floor of Room

s Celing of Room

Walls, Celing. Floor

3 Left wall

S Right. Wall

s Front Wall

Back Wall

$\$$ Floor

s Celing

mode $n$

kcode $3000 \quad 1.010500$

ksrc $0.0 \quad-10.0 \quad-33.0$

$\begin{array}{lll}12.0 & -35.0 & -33.0\end{array}$

$12.0 \cdot 35.0-33.0$

c Cemented waste $(1.024 \mathrm{~g} / \mathrm{cc}) 100 \%$ Water

$\begin{array}{lll}1001.50 \mathrm{C} & -0.1092 & \mathrm{H}\end{array}$

$8016.50 \mathrm{C} \quad-0.8669$

$94239.55 \mathrm{C} \quad-0.0239$

mt 1 poly.01t$$
\text { c }
$$

$c$
$\mathrm{C}$
$\mathrm{m} 2$

$55-304 \quad(7.83 \mathrm{~g} / \mathrm{CC})$

$6000.50 \mathrm{C} \quad 0.000293$

$\begin{array}{rr}14000.50 \mathrm{c} & -0.007497\end{array}$

$\begin{array}{ll}14000.50 \mathrm{C} & -0.007497 \\ 15031.50 \mathrm{C} & -0.000447\end{array}$

$16032.50 \mathrm{C} \quad .0 .000294$

$24000.50 \mathrm{C} \quad .0 .200003$

$25055.50 \mathrm{C} \quad-0.020000$

$26000.55 \mathrm{C} \quad-0.65146$

$28000.50 \mathrm{C} \quad .0 .120002$

C $\quad$ Air $(0.00129 \mathrm{~g} / \mathrm{cc})$

C Alr $(0.00129 \mathrm{~g} / \mathrm{cc})$

$\begin{array}{ll}7014.50 \mathrm{C} & -0.78084 \\ 8016.50 \mathrm{C} & -0.20946\end{array}$

$\begin{array}{rr}8016.50 \mathrm{C} & -0.20946 \\ 18000.35 \mathrm{C} & -0.00934\end{array}$

s 0

c Polyethylene $(0.92 \mathrm{~g} / \mathrm{cc})$

$\begin{array}{llll}\text { m4 } & 6000.50 \mathrm{C} & 0.03952 & \text { s }\end{array}$

$\begin{array}{llll} & 1001.50 \mathrm{c} & 0.07903 & \text { s }\end{array}$

c Concrete $(2.24 \mathrm{~g} / \mathrm{CC})$

$\begin{array}{lcc}c & \text { Concrete }(2.24 \mathrm{~g} / \mathrm{cc}) \\ \text { m5 } & 1001.50 \mathrm{C} & -0.009959\end{array}$

$6000.50 \mathrm{C} \quad-0.000979$

$8016.50 \mathrm{c} \quad-0.529069$

$11023.50 \mathrm{c} \quad 0.016003$

$12000.50 \mathrm{C} \quad-0.002000$

$13027.50 \mathrm{C} \quad-0.034006$

$\$ 4000.50 \mathrm{C} \quad-0.337036$

$19000.50 \mathrm{c} \quad 0.012981$

$20000.50 \mathrm{C} \quad-0.043965$

$26000.55 c$

0.043965

mt5 poly.01t

c Water $(1.0 \mathrm{~g} / \mathrm{cc})$

c Water (1.0 g/CC)

$\begin{array}{ll}1001.50 \mathrm{C} & -0.1119 \\ 8016.50 \mathrm{C} & -0.8881\end{array}$

mt6 lwtr.01t

c $\quad$ Cellulose $(1.45 \mathrm{~g} / \mathrm{Cc})$

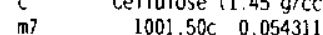

$8016.50 \mathrm{C} \quad 0.026949$

$5 \mathrm{C}$

\$ $\mathrm{Si}$

$\$ P$

$\$ \mathrm{~S}$

S Mn

$5 \mathrm{Fe}$

$5 \mathrm{~N}$

3 ArC

S C

$5 \mathrm{H}$

50

$5 \mathrm{Na}$

$3 \mathrm{Na}$

S Al

S Si

$S K$

s Ca

$5 \mathrm{H}$

50

5 Light water

s Cellulose Reflector 
HNF-6179 Rev. 0

$y=-14$

$y=-13$

$y=-12$

$y=.11$

$y=10$

$y=.9$

$y=-8$

$y=-7$

$y=-6$

$y=-5$

$y=-4$

$y=-3$

$y=-2$

$y=-1$

$y=0$

$y=1$

$y=2$

$y=3$

$y=4$

$y=5$

$y=6$

$y=7$

$y=8$

$y=9$

$y=10$

$y=11$

$y=12$

$y=13$

$y=14$

$y=15$

C

c

c

$y=-14$

$y=-13$

$y=-12$

$y=-11$

$y=-10$

$y=-9$

$y=-8$

$y=\cdot 7$

$y=-6$ $\begin{array}{llllllllllllllll}15 & -13 & -11 & -9 & -7 & -5 & -3 & -1 & 1 & 3 & 5 & 7 & 9 & 11 & 13 & 15\end{array}$

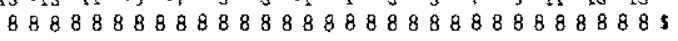
B 8888888888B888888888888888B888s

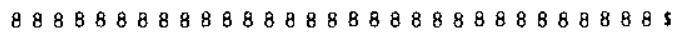
$88888888 B 8888888888888888888888$ s $888888888888 B 888888888688888886$ s $888 B 8888888888888888888886888$ B 5 5 88888888888888888888888888888885 8888888888B88888B8888888886888Bs $8888888888888 B 88888888888888888$ s $8888 B 888898888888$ B 8888888886888 s 8888888888888888888888888888888 s 8888888888888888886888888888888 s 8888888888888888 888888888888888, 8888888868888888888888888888888 s $88888888888888888888888 B 8888888$ s $8888 B 8888 B 8888888 B 88886888 B 8888$ s 8888888888888888888888888888888 s

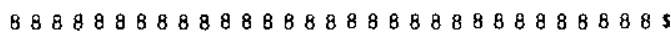
$88888888888888888 B 8888 B 88888 B 88$ s

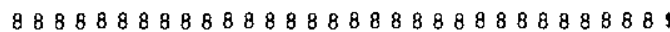
$888888 B 888888888888888888888 B 88$ 8888688888888888888888888888888 s $8888888888888888888888888888 B 88$ s 8888888888888888888888888888888 s 8888888888888888888888888888888 s

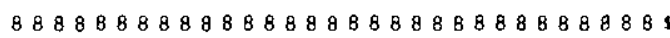
88888888888888888888888888888883 B 888888888888888888888B888B88885 $88 B 8 B 88$ 8B B88888B88888888B8888B8 $8888888888888888888888 B 88886888$ s

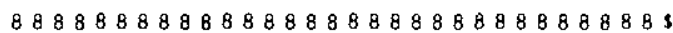

$2=0$

$\begin{array}{lllllllllllllll}-14 & -12 & -10 & -8 & -6 & -4 & -2 & 0 & 2 & 4 & 6 & 8 & 10 & 12 & 14\end{array}$ $\begin{array}{llllllll}3 & 5 & 7 & 9 & 11 & 13 & 15\end{array}$ 8888888888888888888888888888888

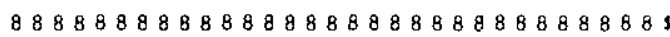
88888888888888888888888888888 8 8888888888888888888888888888888 s 8888888888888888888888888888888 s BBB8BB8B8B8B234238BB888B888888Bs 8888888888884234286888888888888 s 888888888882342342342342348888 8s 8888888888342342342342342388888 s 8888888888234234234234234288888 $y=-5$

$y=-4$

$y=-3$

$y=-2$

$y=\cdot 1$

$y=0$

$y=1$

$y=2$

$y=3$

$y=4$

$y-5$

$y=6$

$y=7$

$y=8$

$y=9$

$y=10$

$y=1]$

$y=12$

$y=13$

$y=14$

$y=15$

$c$

c

$y=-15$

$y=-14$

$y=-13$

$y=\cdot 12$

$y=-11$

$y=-10$

$y=-9$

$y=-8$

$y=-7$

$y=-6$

$y=-5$

$y=-4$

$y=-3$

$y=2$

$y=-1$

$y=0$

$y=1$

$y=2$

$y=?$

$y=4$

$y=5$
88888888884234234234234234888885 8888888888342342342342342388888 s 8888888884234234234234234288888 s 8888888883423423423423423488888 s 8888888882342345342342888888888 s B8886888842342342342348888888B8s 8888883423423423423423888888B88s 8888882342342342342342888868888 s 8888884234234234234238888888888 s 88 88883423423423423428888888888 s $888888234234234234234888 B 888888$ s 8888884234234234234238888888888 s 8888883423423423423428888888888s $88888823423423423423488 B 888888$ s $88888842342342328888888 B 8888888$ s 88 $88883423423423888888888888 \mathrm{~B} 8 \mathrm{~g}$ s 8888882342342342888888888888888 s 8888888884234234888888888888888 s $888888888342342388 B 888888888888$ s B8888B88823423428888888888888885 8886888884234234888688888688888 s

$2=1$

$x=\begin{array}{lllllllllllllll}-14 & -12 & -10 & -8 & -6 & -4 & \cdot 2 & 0 & 2 & 4 & 6 & 8 & 10 & 12 & 14\end{array}$ $\begin{array}{llllllllllllllll}15 & -13 & -11 & -9 & -7 & -5 & -3 & -1 & 1 & 3 & 5 & 7 & 9 & 11 & 13 & 15\end{array}$ 88888888888888888 B 888886888888 8 88 88888888888888888888888888888 $8 B 8888 B 8888888888 B 8888888888888$ s 8888898888888888888888888888888 s 8888888888886888888888888888888 s

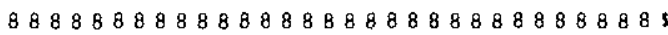
8888888888888888888888888888888 s $88888888888 B 8888868888888888888$ s 8888888888888888888888888888888 s 8888888888888888888888888888888 s $88888888888888 B 8888888888888888$ s

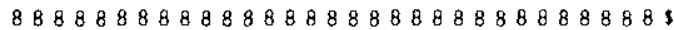
$888868888888888888888 B 888888888$ s $888888868888888888 B 888888888888$ s $88888 B 8888888888888888888888888$ s $88 B 88888888888888888888888888885$ B8B888BB8888B88888B8B888B8888B85 $88 B 8888888888 B 88888888888 B 88888$ s 888888888888888888888888888888 s 8888888888888888888888888888888 s $88 B 8898888888 B 88888888888 B 88888$ 


\section{HNF-6179 Rev. 0}

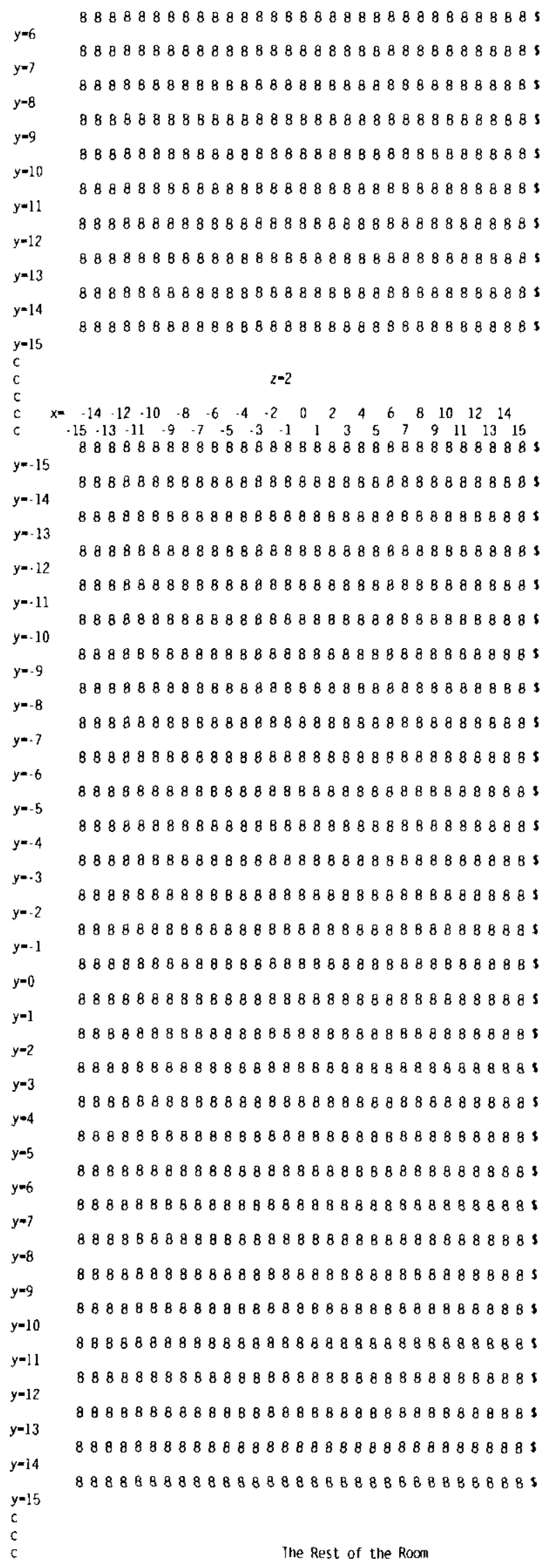

$y=15$
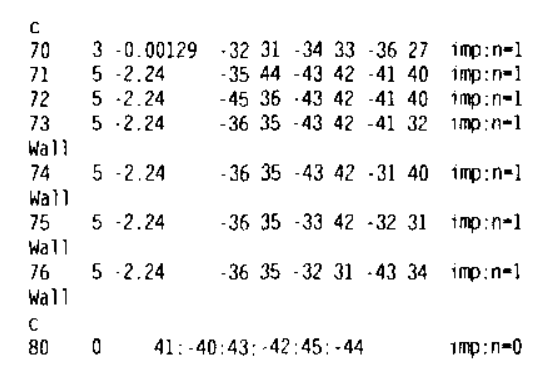

$\begin{array}{lll}c & & \text { Surface Definitions } \\ c & & \text { Billets } \\ c & & \end{array}$

$\begin{array}{rrr}1 & c z & 6.985 \\ 2 & p 2 & -8.890 \\ 3 & p 2 & 8.890 \\ 4 & C 2 & 6.988 \\ 5 & p 2 & -8.893\end{array}$

5
6
$c$
7

$$
\begin{aligned}
& 7 \\
& \text { Int } \\
& 8 \\
& \text { Int } \\
& 9 \\
& \mathrm{C} \\
& 10 \\
& 11 \\
& 12
\end{aligned}
$$$$
\begin{aligned}
& 10 \\
& 11 \\
& 12 \\
& c \\
& 13
\end{aligned}
$$$$
14
$$$$
\begin{aligned}
& 15 \\
& 16 \\
& c \\
& 17
\end{aligned}
$$

$$
\begin{aligned}
& 14 \\
& 15 \\
& 16 \\
& c \\
& 17 \\
& c \\
& c \\
& 20 \\
& 21
\end{aligned}
$$$$
2
$$$$
\begin{aligned}
& 21 \\
& c \\
& 22 \\
& 23
\end{aligned}
$$

1

$$
\begin{aligned}
& 22 \\
& 23 \\
& 24 \\
& 25 \\
& c \\
& c \\
& 26
\end{aligned}
$$$$
\begin{aligned}
& \text { volume } \\
& c \\
& c
\end{aligned}
$$$$
42.3795
$$$$
\text { volume }
$$$$
31 \text { Room }
$$$$
32 \mathrm{px}
$$$$
\text { Room }
$$$$
\begin{aligned}
& \text { Room } \\
& 33 \\
& 34
\end{aligned}
$$$$
\begin{array}{ll}
34 \\
35
\end{array}
$$$$
\begin{aligned}
& c \\
& 40
\end{aligned}
$$$$
\begin{array}{ll}
c & \\
40 & p x \\
41 & p x \\
42 & p y \\
43 & p y \\
44 & p 2 \\
45 & p 2
\end{array}
$$$$
\text { orum }
$$

Radius of Waste

Botton of waste

5 Top of Waste

s Outside of Can

- Top of Can

S Radius of

s Bottom of

5 Top of Interior

$\$$ Radius of Liner s Bottom of Liner $\checkmark$ Top of Liner

s Radius of Drimn - Botton of Orum 5 Top of Drum

5 Top above Drum

s $\mathrm{Pu}$ in other drun

$\mathrm{px}$
$\mathrm{px}$

px $\quad 28.729$

\section{mode $n$}

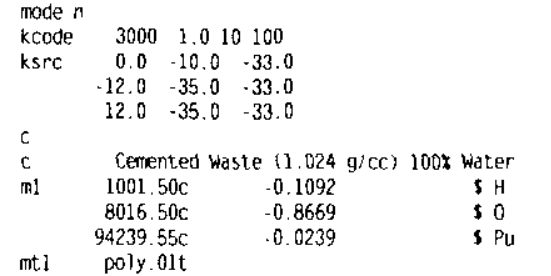

$B y+C z=0)$ 
HNF-6179 Rev. 0

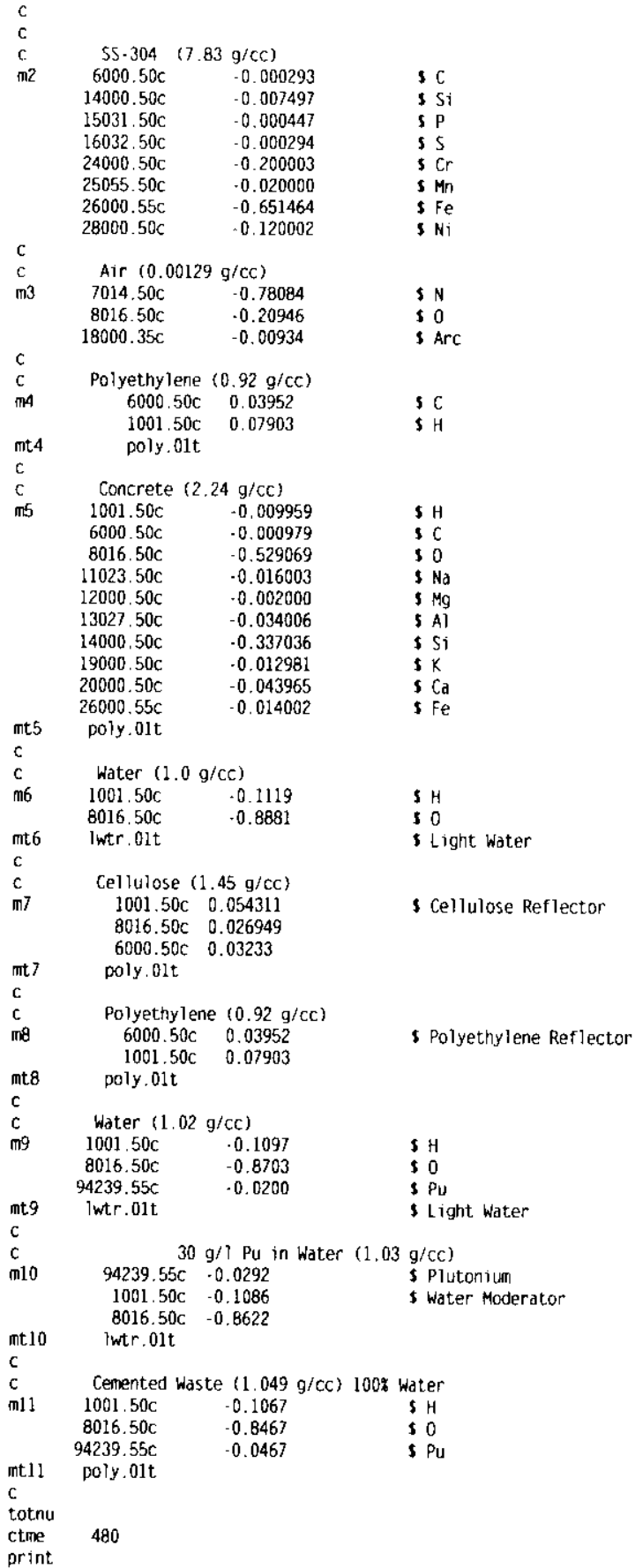

nonpipe_\}at4 inp

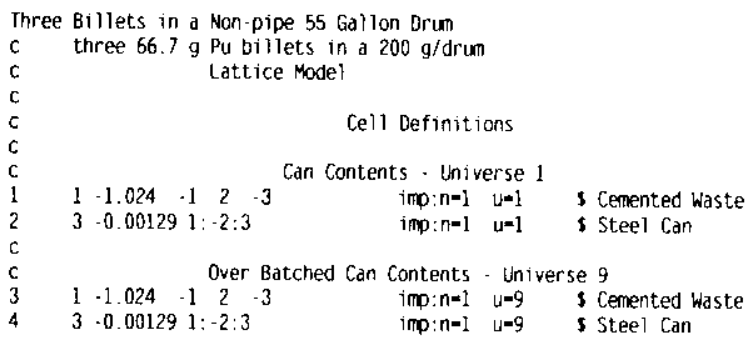

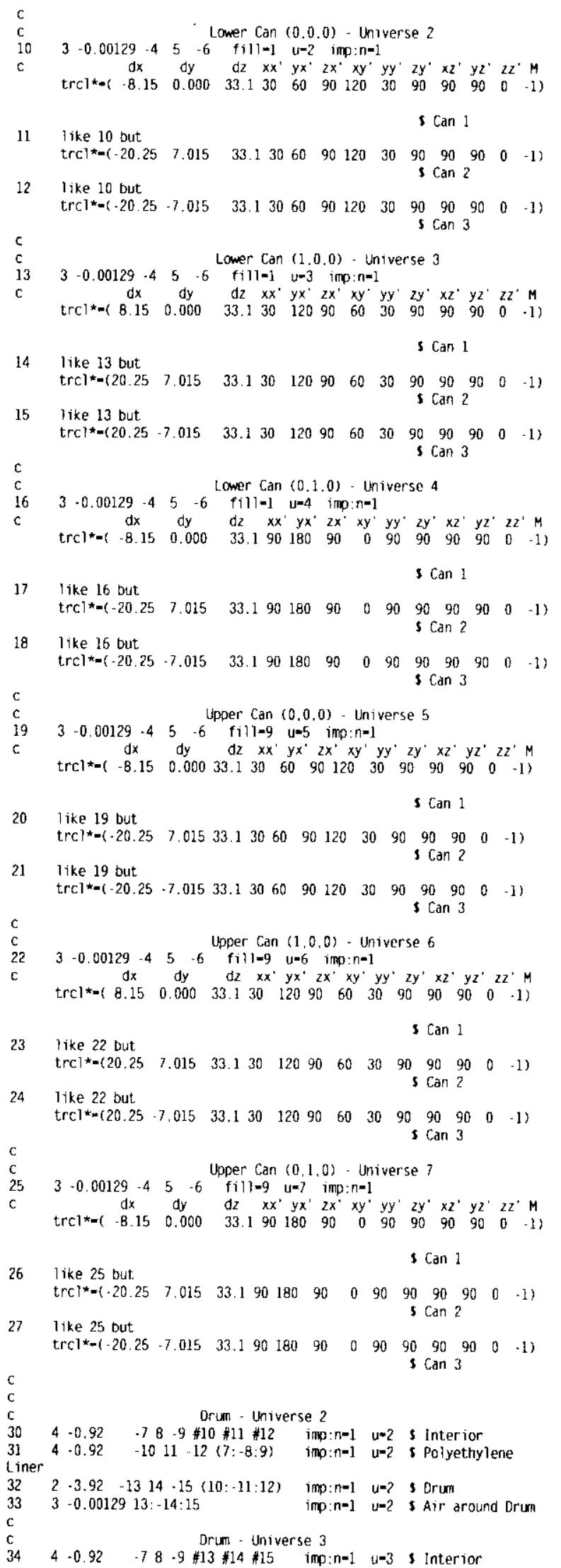




\section{HNF-6179 Rev. 0}

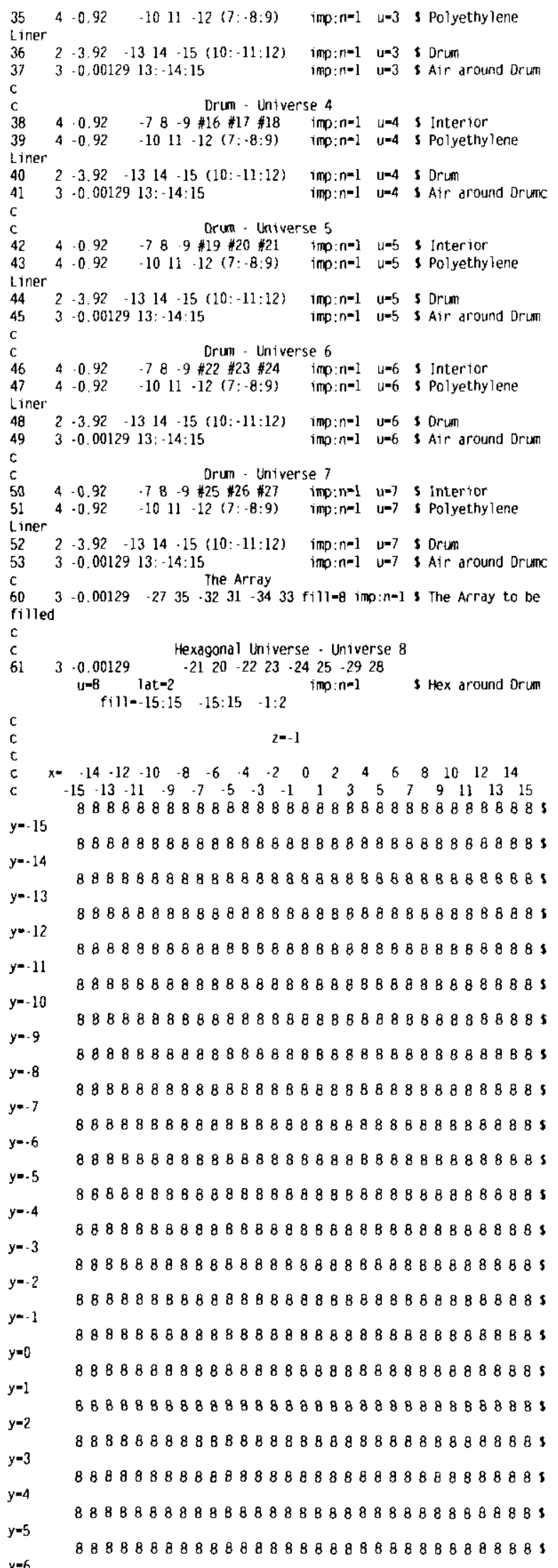

$y=7$

$y=8$

$y=9$

$y=10$

$y=11$

$y=12$

$y=13$

$y=14$

$y=15$

c

c C

$y=-15$

$y=-14$

$y=-13$

$y=-12$

$y=-11$

$y=-10$

$y=-9$

$y=-8$

$y=-7$

$y=-6$

$y=-5$

$y=-4$

$y=-3$

$y=-2$

$y=-1$

$y=0$

$y=1$

$y+2$

$y=3$

$y=4$

$y=5$

$y=6$

$y=7$

$y=8$

$y=9$

$y=10$

$y=11$

$y=12$

$y=13$

$y=14$

$y=15$

$c$
$8 B 888888888888888888888888888885$ $888888888 B 8888888 B 88888888888 B 8$ s 88888888888888888888888888888885 8889888888888888888888868888888 s $888888888888888 B 88888888888 B 888$, 88888888888888888888888888888885 88888888888888888488888888888885 $888888888888 B 888886888888888888$ s

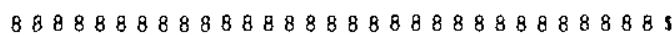

$z=0$

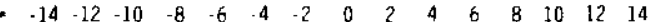
$\begin{array}{cccccccccccccccc}15 & -13 & -11 & -9 & -7 & -5 & -3 & -1 & 1 & 3 & 5 & 7 & 9 & 11 & 13 & 15\end{array}$ 898888888888888 B 888888888888888 s

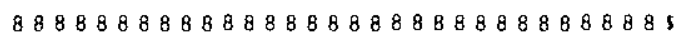
8688888888888888888888888888888 , 8888888888888888888888888888888

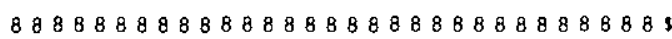
86888888888823423888888888888885 88888888888842342888888888888885 88888888888234234234234234888883 8888888888342342342342342388888 s 88888888882342342342342342888885 $88888888 B 84234234234234234888885$ 8888888888342342342342342388888 s B888888884234234234234234288888s 8888888883423423423423423488888 s 888888888234234564234288888888 s, B8888B88842342372342348888888885 8888883423423423423423888888888s 8888882342342342342342888888888 s 8888684234234234234238888888888 s 8888883423423423423428888888888 s 8888882342342342342348888888688 88868842342342342342388888888885 88888834234234234234288888888 B 8s 88888823423423423423488888888885 8886884234234232888888888888888 s $8888883423423423886888888888 B 88$ s 8888882342342342888888888888888 s 8888888884234234888888888888888 s B8888B8883423423888888888888888, 8888888882342342888688888888888

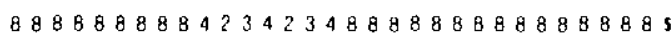

$2=1$

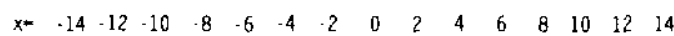


$y=-14$

$y=-13$

$y=12$

$y=-11$

$y=\cdot 10$

$y=-9$

$y=-8$

$y=-7$

$y=6$

$y=-5$

$y=-4$

$y=-3$

$y=-2$

$y=-1$

$y=0$

$y=1$

$y=2$

$y=3$

$y=4$

$y=5$

$y=6$

$y=7$

$y=8$

$y=9$

$y=10$

$y=11$

$y=12$

$y=13$

$y=14$

$y=15$

c

$\mathrm{c}$

c

$y=\cdot 15$

$y=-14$

$y=-13$

$y=12$

$y=-11$

$y=-10$

$y=-9$

$y=-8$

$y=-7$

$y=-6$

$y=-5$

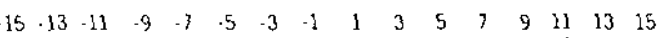
88 B8888 B888888888888888888888885

88888888888888888888888888888885

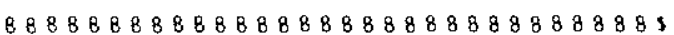

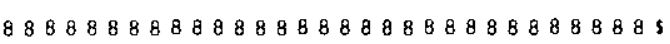

$8888888888888888888888888888888 \mathrm{~s}$

8888888888888888888888888888888 s

$88888888888888 B 88888888888888885$

$88888 B 8888888 B 888888888 B 88 B 8 B B 85$

$8888888888888888 B 888888888888 B 8$ s

88888888888888888888888888888 \& 3

888888888888888888B88888888B8885

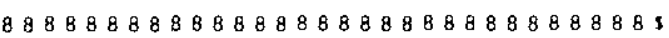

$8 B 888888 B 888888888 B B 888888888885$

B 8888B8888888888B B88888888B88885

$88888888888888888888 B 8888888888$ s

$8 B 888888 B 8888885688888888888888 \mathrm{~s}$

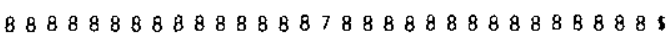

8888888888888888888888888888888 s

8 $8888888 B 88888 B 8888 B 88888888888$ s

$88 B 888 B 8888888888888888888 B 8888 \mathrm{~s}$

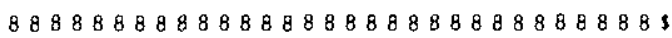

$8 B 88888 B 888$ 8 88888888888888888885

88888888888888888888888888888885

$88 B 888888 B 88888 B 888 B 888888888 B 8$ s

$88 B 8888888888 B 888888888 B 888 B 8885$

B8888B88 g888888888888888888888Bs

8 $88 B 8888 B 88888888$ 8 $88888 B 8888$ B s

$8888888 B 88888888888888888888888 \mathrm{~s}$

88 $88 B 88888888888848888888 B 88888$ s

$88 B 88888888888888888888 B 8888 B 885$

B88888B8888888888888888888988885

$z=2$

$\begin{array}{llllllllllllllll}-14 & 12 & -10 & 8 & -6 & -4 & -2 & 0 & 2 & 4 & 6 & 8 & 10 & 12 & 14\end{array}$ $\begin{array}{llllllllllllllll}15 & 13 & -11 & -9 & -7 & -5 & -3 & -1 & 1 & 3 & 5 & 7 & 9 & 11 & 13 & 15\end{array}$ 8888888888888888888888888888888 s

8888888888888888888888888888888 s

$8888888888888888888888888888888 \mathrm{~s}$

888888888888 88888888888888888885

88888888888888888888888888888885

$88 B 888888888888888888 B 8888888 \mathrm{BB}$ s

$888888888888888888888888888 B 888$ s

8888888888888888888888888888888

88 88888888888888B88888B888888885

88888888888888888888888888888885

88 B 88888888888888888888888888B8s $y=-4$

$y=3$

$y=-2$

$y=-1$

$y=0$

$y=1$

$y=2$

$y=3$

$y=4$

$y=5$

$y=6$

$y=7$

$y=8$

$y=9$

$y=10$

$y=11$

$y=12$

$y=13$

$y=14$

$y=15$

$$
70
$$

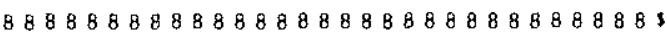
88 88888688888888888888888988888 s $8 B 888888888888888888888886888885$ 8888888888888888888888888888888

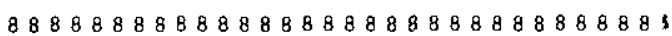
8888888888888888888888898888888 s B88888888B888888B888888888888B85 8888888888888 B88888B888888888885 8888888888888888888888888688888 s $8888888888888888888888888888 B 88$ s $88 B 8 B B 88888888888888888888888885$ $8 B 88888888888888888888888888888$ s

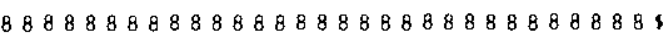
$888888888 B 888888888888 B 888888885$ 88888888888888888888888888 B8888s $88888 B 8 B 88888 B 8 B 888888888888888$ s $88888888888888 B 8888888888888888$ s 8888888888888888848888888888888 s B 8888888888888888888888888888 8

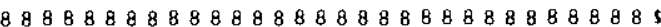

\section{The Rest of the Room} $\begin{array}{llllllll}-36 & 35 & -33 & 42 & -32 & 31 & \text { imp: } n=1\end{array}$ $-2.24$ $\begin{array}{lllllll}36 & 35 & -32 & 31 & -43 & 34 & \text { Imp:n-1 }\end{array}$ $41:-40: 43:-42: 45:-44 \quad$ imp: $n=0$ Surface Definitions

Billets

$\begin{array}{lllllll}31 & -34 & 33 & -36 & 27 & \text { imp: } n-1\end{array}$ $\begin{array}{llllllll}5 & 44 & -43 & 42 & -41 & 40 & \text { imo:n-1 }\end{array}$ $\begin{array}{llllllll}45 & 36 & -43 & 42 & -41 & 40 & \text { imp: } n=1\end{array}$ $\begin{array}{llllllll}-45 & 36 & -43 & 42 & -41 & 40 & \text { imp: } n=1 \\ -36 & 35 & -43 & 42 & -41 & 32 & \text { imp: } n=1\end{array}$

- Air above array Concrete Celing s Right Concrete

- t.eft Concrete

s Front Concrete

S Back Concrete

28.485

$-41.999$

41.999

28.575

28.575
-42.227

-42.227
42.227

28.727

$-42.379$

42.379

42.380

15.00

px $\quad-28.729$

$p x$
Drun

Botton of Waste

Bottom of Waste

S Top of Waste

Outside of Can

$\checkmark$ Bottom of Can

s Top of Can

5 Radius of

5 Botton of

5 Top of Interior

3 Radius of liner 3 Bottan of Liner s Top of Liner

5 Radius of Drum 5 Bottom of Drum 5 Top of Drum

s Top above Drum

5 Pu in other drum

Surfaces $(A x+B y+C z=0)$
Hexagonal Surfaces 
HNF-6179 Rev. 0

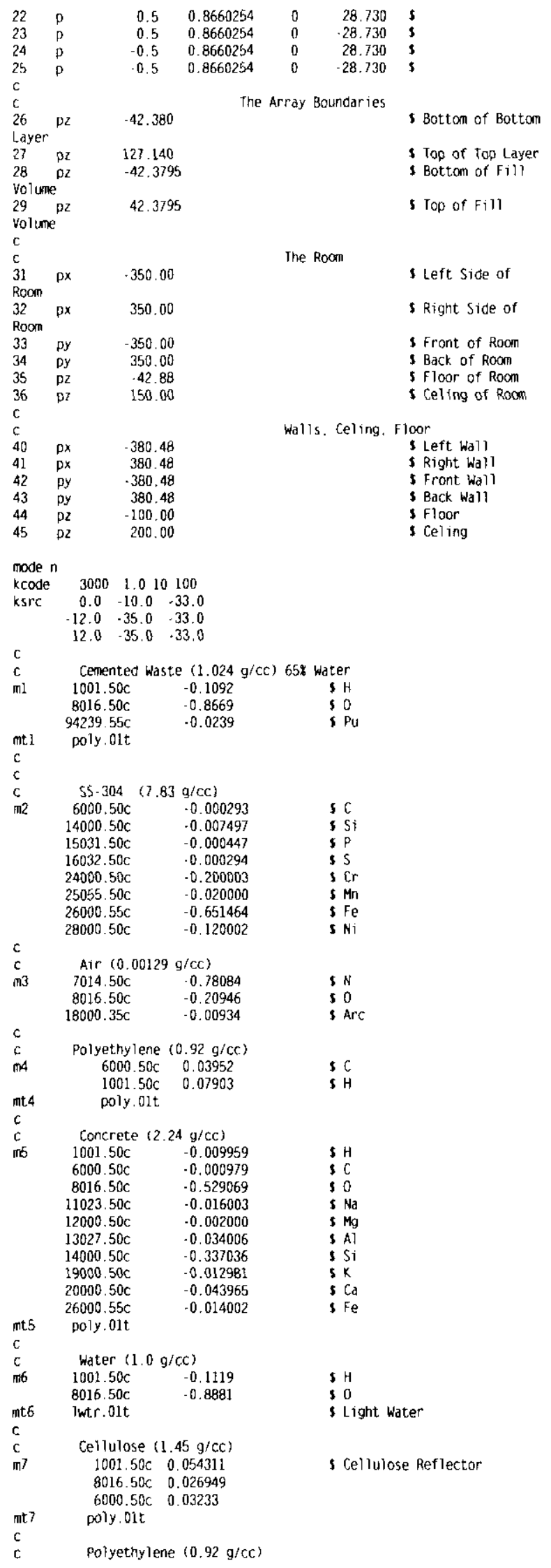

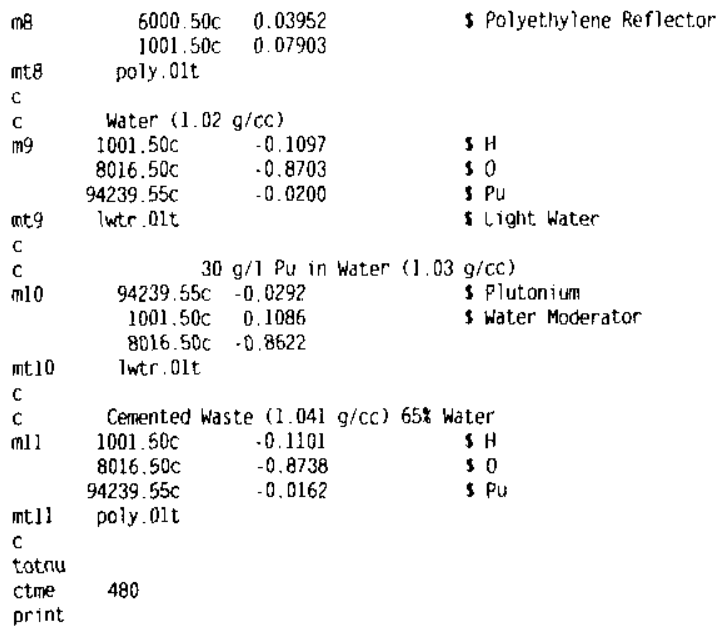

nonpipe_lats.inp

Three Billets in a Non-pipe 55 Gallon Drum

three $66.7 \mathrm{~g}$ Pu billets in a $200 \mathrm{~g} / \mathrm{drum}$ Lattice Model with Wagon

\section{Cell Definitions}

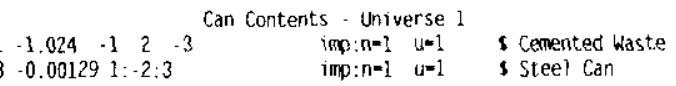

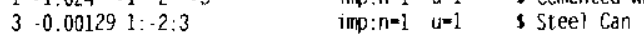

Over Batched Can Contents - Universe

$11-1.041-1 \quad 2 \quad-3 \quad$ imp: $n=1 \quad \mu=9$ s Cemented Waste

$3.0 .001291:-2: 3 \quad$ imp: $n=1, w=9$ S Steel Can

Wagon Can Contents. Universe 10

8 $-11.46 \quad-208204 \cdot 2.05 \quad u=10 \quad$ imp: $n=1 \quad$ s Plutonium $0 x$ ide $3-0.00129$ 201:-204:206 u=10 imp:n=1 s Can

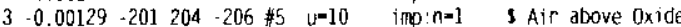

Lower Can $(0,0,0)$ - Universe ?

$\begin{array}{llllllll}3 & -0.00129 & -4 & 5 & -6 & \text { fill-1 } & \mathrm{y}=2 & \text { ino: } n-1\end{array}$

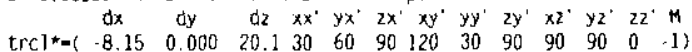

s can 1

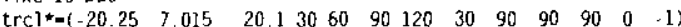

$3 \operatorname{Can} 2$

12 like 10 but

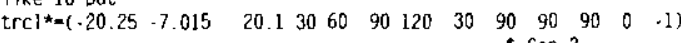

s can 3

c Lower Can $(1,0.0)$ - Universe 3

$13 \quad 3-0.00129-4 \quad 5 \quad-6 \quad$ fill $=1 \quad u=3$ imp: $n=1$

c $d x \quad d y \quad d z \quad x x^{\circ} y x^{\circ} 2 x^{\circ} x y^{\prime} y y^{\circ} z y^{\prime} x z^{\prime} y z^{\prime} z z^{\prime} M$

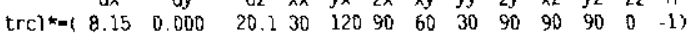

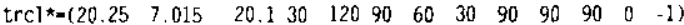

S Can 2

15 like 13 but

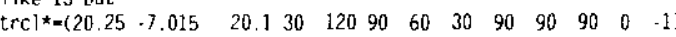
S Can 3

c Lower Can $(0.1,0)$. Universe 4

$16 \quad 3 \cdot 0.00129-4 \quad 5 \quad-6 \quad$ filli=l $u=4$ imp:n=1

$d x \quad d y \quad d z \quad x x^{\prime} y x^{\prime} 2 x^{\circ} x y^{\prime} y y^{\prime} z y^{\prime} x z^{\prime} y z^{\prime} 2 z^{\prime} M$

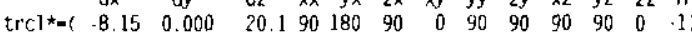

s Can 1

$17 \quad$ like 16 but

$\operatorname{trci})^{*}\left(\begin{array}{ccccccccccccc}-20.25 & 7.015 & 20.1 & 90 & 180 & 90 & 0 & 90 & 90 & 90 & 90 & 0 & -1\end{array}\right)$

$5 \operatorname{can} 2$

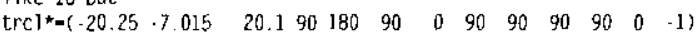
5 Can 3

c Upper Can $(0.0 .0)$ - Universe 5

$19 \quad 3-0.00129-4 \quad 5 \quad-6$ fill-g ums ifno: $n=1$ $d x$ dy dz $x x^{\prime} y x^{\prime} z x^{\circ} x y^{\prime} y y^{\prime} z y^{\prime} x z^{\prime} y z^{\prime} z z^{\prime} M$ 
$\operatorname{trc}^{*}=\left\{\begin{array}{rllllllllllll}-8.15 & 0.000 & 20.1 & 30 & 60 & 90 & 120 & 30 & 90 & 90 & 90 & 0 & -1\end{array}\right)$

like 19 but

- Can 1

s Can 2

\section{like 19 but}

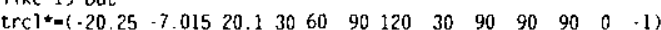
$\checkmark \operatorname{Can} 3$

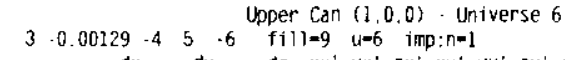

$d x \quad d y \quad d z \quad x x^{\prime} y x^{\prime} z x^{\prime} x y^{\prime} y y^{\prime} z y^{\prime} x z^{\prime} y z^{\prime} z z^{\prime} M$

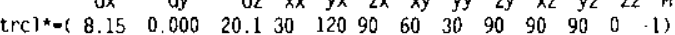

s can 1

\section{like 22 but}

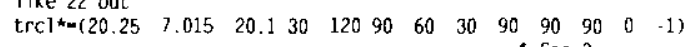

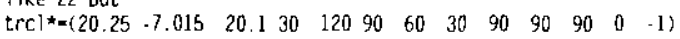

5 Can 3

$\begin{array}{llllll} & -0.00129 & -4 & 5 & -6 & \text { Upper Can }(0.1,0)\end{array}$

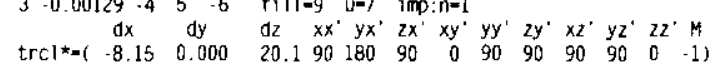

like 25 but

s Can 1

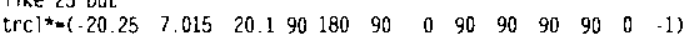

like 25 but

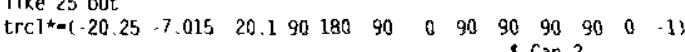
san 3

$$
\begin{gathered}
\text { Drum - Universe } 2 \\
4-0.92 \quad-78-9+010,11-412
\end{gathered}
$$

$4-0.92 \quad-78 \quad-9$ \#10 \#11 \#12 imp:n=1 u=2 s Interior

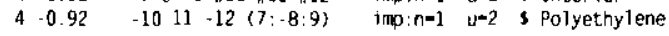

$2-3.92-13 \quad 14-15(10:-11: 12)$ imp:n-1 $u=2$ s Drum

$\begin{array}{lll}3-0.0012913:-14: 15 & \text { imp:n=1 } u=2 \text { s Air around Drum }\end{array}$

\section{Drum - Universe 3}

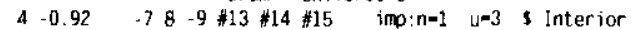

$4-0.92 \quad-1011-12(7:-8: 9) \quad$ imp:n=1 $\quad u=3$ s Polyethylene

$2 \cdot 3.92-1314 \cdot 15(10 \cdot-11: 12)$ imp:nml u=3 s Orum

$3 \cdot 0.00129$ 13:-24:15 imp:n-1 u=3 3 Air around Drum

Drum - Universe 4

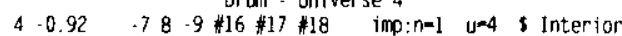

$4-0.92 \quad-1011-12(7:-8: 9) \quad$ imp:n-1 $u=4$ s Polyethylene

ner

$2-3.92 \cdot 1314-15(10:-11: 12)$ imo:n-1 ume s Drum

$3 \cdot 0.00129$ 13:-14:15 imp:n=1 $u=4$ sir around Drunc

$\begin{array}{lll}4.0 .92 \quad-78.9 & \text { Drum - Universe } 5\end{array}$

8.9 \$19 *20 \#21 imp:n-1 u=5 s Interior

$.1011-12(7:-8: 9)$ imp:n-1 u-5 5 Polyethylene

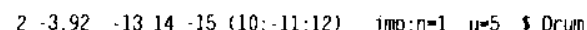

3. $\cdot 0.0012913: \cdot 14: 15$ imp:n=1 $\mathrm{u}=5$ s Air around Orum

$\begin{array}{llll}4 & -0.92 \quad-78-9 & \text { Orum - Universe } 6\end{array}$

$4-0.92 \quad-78-9 \quad \# 22$ \#23 $\# 24 \quad$ imp: $n=1 \quad u=6$ s Interior

$2-3.92-13 \quad 14-15(10:-11: 12)$ imp:n-1 umb sorum

$3-0.0012913:-14: 15$ imp:n=1 $u=6$ \& Air around Drum

Drum - Universe 7

-7 8 -9 \#25 \#26 \#27 imp:n-1 u-7 s interior

ner

$2 \cdot 3.92-1314 \cdot 15(10 \cdot \cdot 11: 12) \quad$ imp:n-1 u-7 s Drum

3 - 0.00129 13:-14:15 imo:n=1 $u=7$ s Air around Orumc

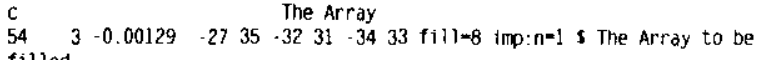

c

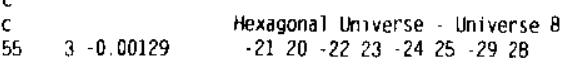

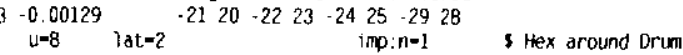
fi $11--15: 15 \quad-15: 15 \quad-1: 2$

$z=-1$

$x=\begin{array}{lllllllllllllll}-14 & -12 & -10 & -8 & -6 & -4 & -2 & 0 & 2 & 4 & 6 & 8 & 10 & 12 & 14\end{array}$
$88888888888888888888888888888 B 8$ s 88888888888888888888888888888885 88888 B 888888 B 88888888888888888 Bs

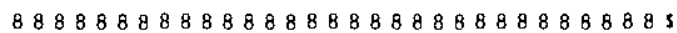
$888888 B 88888888889868 B 88 B B 88 B 885$

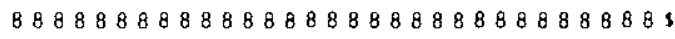

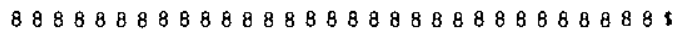
8888888888888888888888888888888 s 888888888 B B88888B888888888888885 88888888888888888888888888888885 $88888888888888 B 88888888888888885$ 88888888886888886888888888888885

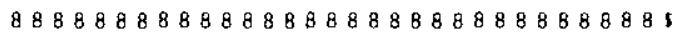
8888888888888888888888888888888 s $8888 B 8888888888 B 88888$ B88888B8885 $8888888688888888888 B 88888888888$ s $8888888 B 88888888888888888888888 \mathrm{~s}$ $8888 B 88888 B 888 B 888888888888$ B8885 88888888888888888888888888888865 $8888 B 88888888888 B 888888888888885$ $88 B 8888888888888888888888888888$ s

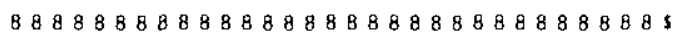
8 888888888888888888888 888888886s

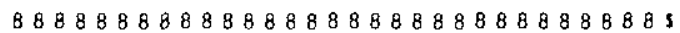
$88888888888888888888888888 B 8898$ s $88888888888888888888888888888 B 8$ s B88888888888888888888888888888885 88888888888888888888888888 88888s $888888888888888884888 B 8888888885$ 8888888888888888888888888888888 s 8888888888888888888888888888888 s

$z=0$

$x=\begin{array}{lllllllllllllll}14 & -12 & -10 & -8 & -6 & -4 & -2 & 0 & 2 & 4 & 6 & 8 & 10 & 12 & 14\end{array}$ $8 B 8888888888 B 88888 B 88$ 8 888888 B 68 , $y=-15$ 88888888888888888888888888888885 8888888888888888888888888888888 s 8888888888888888888888888888888 s 88888888888888888888888888888885

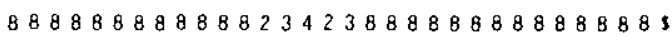
$8 B 88888888884234288888888 B 888885$ 888888888882342342342342348BB है क 8888688888342342342342342388888 s 8888888888234234234234234288888 s 8888888888423423423423423488888 s 
HNF-6179 Rev. 0

88888888883423423423423423888865 $88 B 8888884234234234234234288888$ s

$y=-3$

$y=-2$

$y=-1$

$y=0$

$y=1$

$y=2$

$y=3$

$y=4$

$y=5$

$y-6$

$y=7$

$y=8$

$y=9$

$y=10$

$y=11$

$y=12$

$y=13$

$y=14$

$y=15$

C

$y=-15$

$y=-14$

$y=-13$

$y=-12$

$y=-11$

$y=-10$

$y=-9$

$y=-8$

$y=-7$

$y=-6$

$y=-5$

$y=-4$

$y=-3$

$y=-2$

$y=-1$

$y=0$

$y=1$

$y=2$

$y=3$

$y=4$

$y=5$

$y=6$

B 8888888834234234234234234888885

88888888823423423423428888888885 $88888 B 88842342342342348888888885$ 888888342342342342342388888888 s 88888823423423423423428888888885 88888842342342342342388888868885 $88888 B 3423423423423428888888888$ s 88888823423423423423488888 B8888s $88 B 88842342342342342388888888885$ $8 B 88883423423423423428888888888$ s $8888882342342342342348888888888 \mathrm{~s}$ 8888884234234232888888888B888B8s 88888834234234238888888868888885 88888823423423428888888888888885 $88888 B 88842342348888888888888885$ $8888888883423423888888888888888 \mathrm{~s}$ 8868888882342342888 B888888888885 $8 B 88888 B 8423423488 B 888888888 B 88$ s

$2+1$

$\begin{array}{lllllllllllllll}-14 & -12 & -10 & -8 & -6 & -4 & -2 & 0 & 2 & 4 & 6 & 8 & 10 & 12 & 14\end{array}$ $\begin{array}{llllllllllllllll}5 & -13 & -11 & -9 & -7 & -5 & -3 & -1 & 1 & 3 & 5 & 7 & 9 & 11 & 13 & 15\end{array}$ 88888888888888888888888888868885 B8888888888888888888888888B888BS 88 888888888888888888888888 88888s $88 B 8888888888888888888868888888$ s

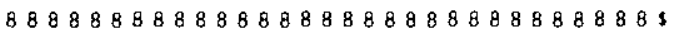
8 8888888888888888888 8 8888888888s $8 B 88888 B 8888868888 B 888888888888$ s 888888888888888888888 B88888888Bs 8 $88888888 B 888888888888886888888$ s $8 B 88888888888888888888888888888$ 5 $88888 B 88888 B 888888888888888888 B 5$

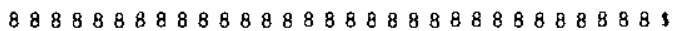
88888888888888888888888888888885 $888888888888888888888888888888 B$ s $88 B 8688888888888888888888888888$ s 88888888688888888888888888888885 $888888 B 888888888868888888888888$ s 8888888888888888888888888888888 s 8888 8 88888888888888688888888888 s

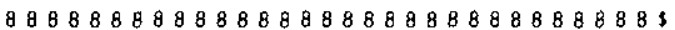
$8 B 88888888888888888888888888888$ s $8 B 88888888888688888888888888 B 885$ $y=7$

$68888 B 8888888888888 B 888888688885$ 88888888888888888888888888888883 888688888888868888888 B B 888888885 $y=9$ $y=10$ $y=11$ $y=12$ $y=13$ $y=14$ $y=15$$$
\text { c }
$$$$
\text { c }
$$$$
c
$$

$y=-15$

$y=-14$

$y=-13$

$y=-12$

$y+11$

$y=-10$

$y=-9$

$y=-8$

$y=-7$

$y=-6$

$y=-5$

$y=-4$

$y=-3$

$y=-2$

$y=-1$

$y=0$

$y=1$

$y=2$

$y=3$

$y=4$

$y=5$

$y=6$

$y=7$

$y=8$

$y=9$

$y=10$

$y=11$

$y=12$

$y=13$

$y=14$

$y=15$

88888888888688888888888888888885 $8 B 88888 B 88 B B 8 B 888 B 8 B 8 B B 8 B 8 B B B B 85$

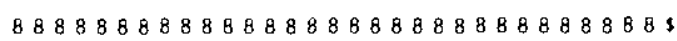

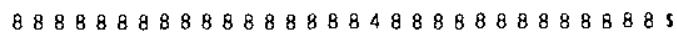
8888888688888 B88888888888888888 $8 B 88888 B 88888888888888888888888$,

$z=2$

$\begin{array}{lllllllllllllll}-14 & -12 & -10 & -8 & -6 & -4 & -2 & 0 & 2 & 4 & 6 & 8 & 10 & 12 & 14\end{array}$ $\begin{array}{llllllllllllllll}15 & -13 & -11 & -9 & -7 & -5 & -3 & -1 & 1 & 3 & 5 & 7 & 9 & 11 & 13 & 15\end{array}$

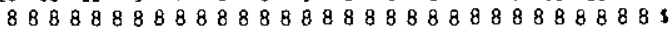
8888888888888888888888888888888 s

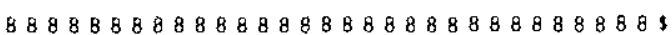

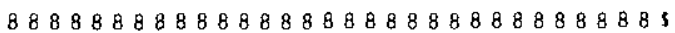
8 $8888888 B 888886888888888888888$ s 88888888888888888888888888888885 8888888888888888888888888888888 ,

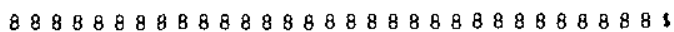
$888888 B 8 B B 8 B 8 B B 88888888888888885$

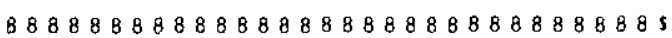

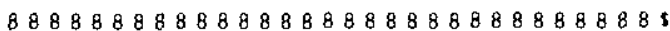
A8888888888888888888888888888885

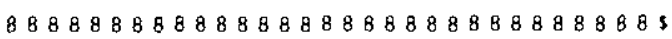

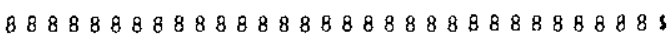
8888888888888B88888888888B888883 B B 8 8 $88 B 888888888888888688888888$ s

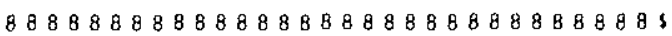
$88 B 8888688888888888888888888888$ s

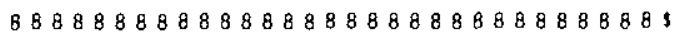
888888888888888888888888888888 8 g 888888 B B 8888B8888888888B88888B5 8888888888888888888888888888888 s $888888888 B 888888888888888888888$ s

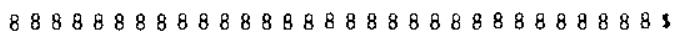
$88888888888 B 888888888868888888$ s $8888 B 888888888 B 8888888888888888$ s 888888888888888888888888888888 B 8888886888888888888888688888888 s $88888888888888888488888888 B 8888$ s 8 88888888888888888888888 888888 s 88888888888868888888888888888885

Cans in wagon

$3-0.00129-202203-207$ fill $=10 \quad$ imp: $n=1$ trcl- $-1.24 .158-360.529-19.200)$

s Wagon Can \#1 
HNF-6179 Rev. 0

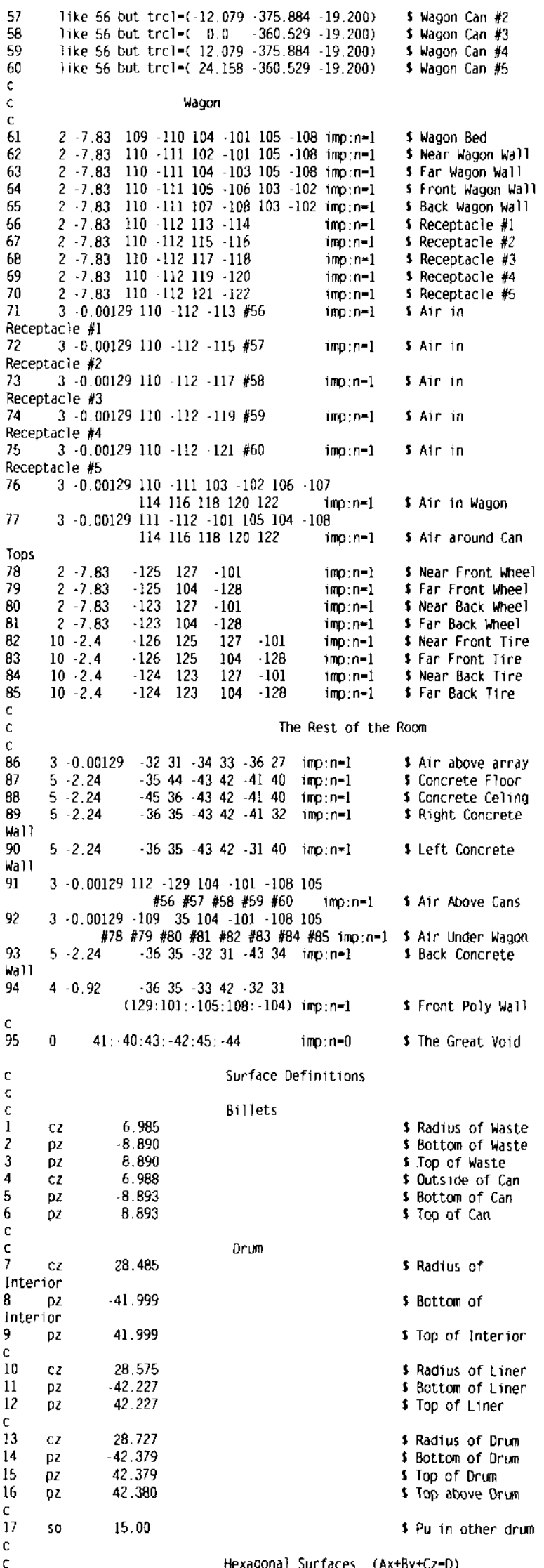

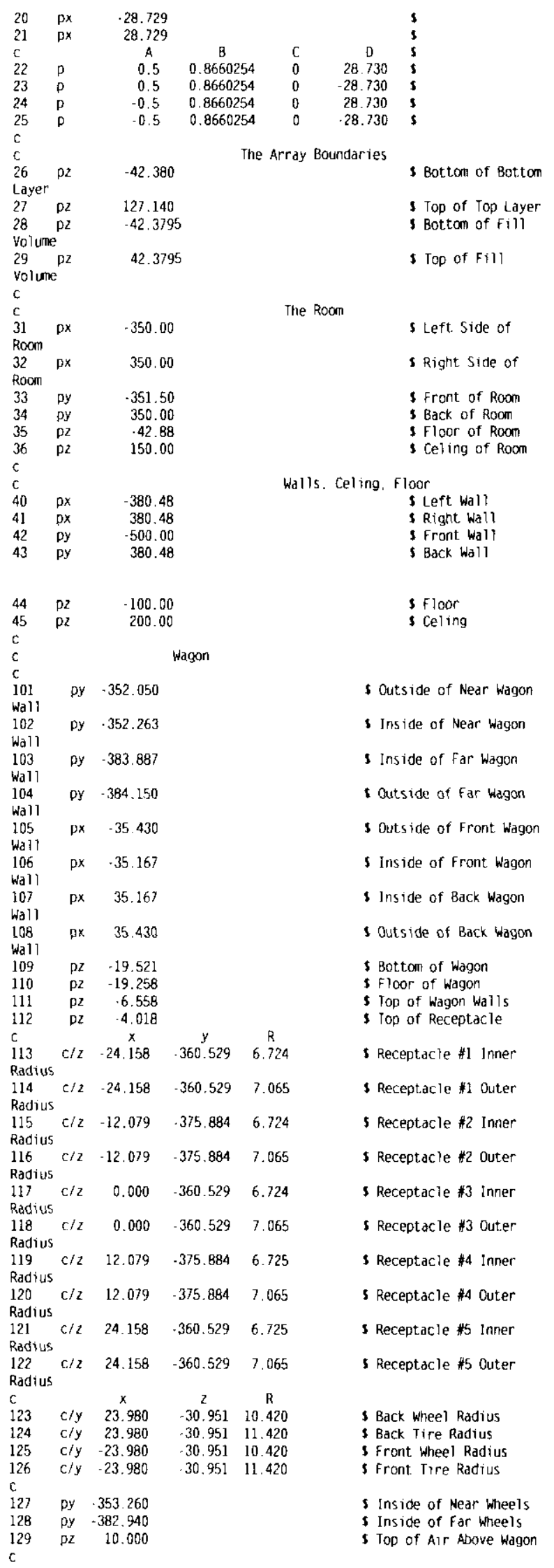




\section{HNF-6179 Rev. 0}

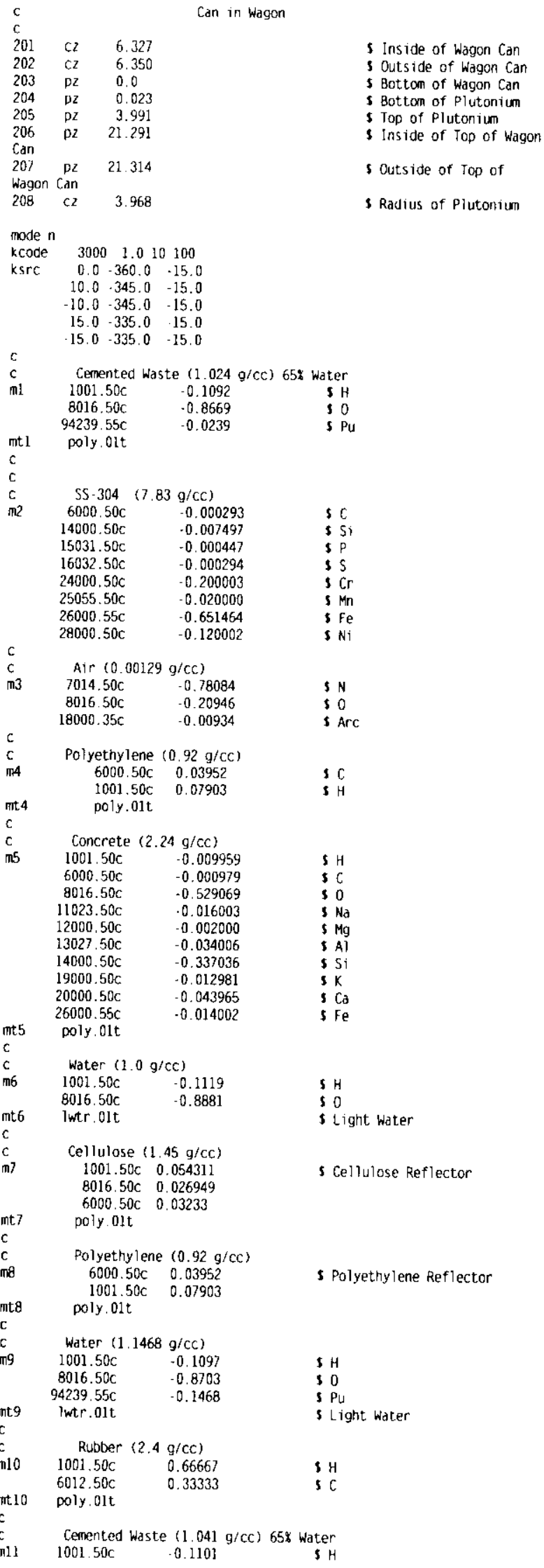

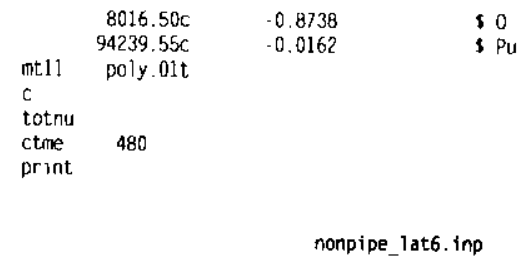

Three Billets in a Non-pipe 55 Gallon Orum three $66.7 \mathrm{~g}$ Pu billets in a $200 \mathrm{~g} / \mathrm{drum}$ Lattice Model with wagon

Cell Definitions

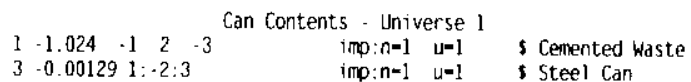
Over Batched Can Contents. Universe 9 $\begin{array}{llllll}1 & 1.024-1 & 2 & -3 & 3 & \end{array}$ $3-0.001291:-2: 3 \quad$ imo:n-1 $u=9$ s Steel Can

1 Wagon Can Contents - Universe 10

$11-19.6 \quad-208204-205 \quad u=10 \quad$ imo: $n-1$ s Plutoniun 0xide $3-0.00129$ 201:-204:206 $u=10$ imp:n=1 S Can

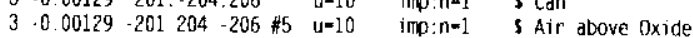
$3-0.00129-4$ Lower Can $(0.0,0)$ - Universe 2 $\begin{array}{lllllllll}3 & -0.00129 & -4 & 5 & -6 & \text { fill } & u-2 & \text { imp }: n=1\end{array}$

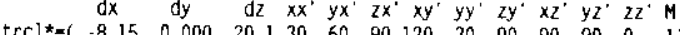


$\left.\begin{array}{lllllllllllll}\text { like } 22 \text { but } & \\ \operatorname{trc})^{*}=(20.25 & -7.015 & 20.1 & 30 & 120 & 90 & 60 & 30 & 90 & 90 & 90 & 0 & -1\end{array}\right)$ 5 Car 3

c. Upper Can $(0,1,0)$ - Universe

$\begin{array}{lllllll}3-0.00129 & -4 & -6 & \text { f1ll-9 } & u-7 & \text { imp: } n=1\end{array}$

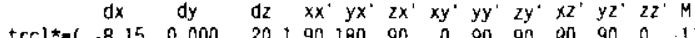

like 25 but

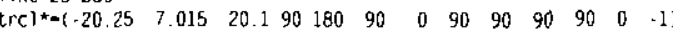

s Cari 1

$27 \quad$ like 25 but

$\left.\begin{array}{lllllllllllll}\operatorname{trc} l^{*}=(-20.25 & -7.015 & 20.1 & 90 & 180 & 90 & 0 & 90 & 90 & 90 & 90 & 0 & .1\end{array}\right)$ s Car 3

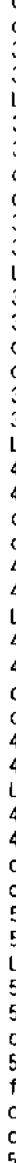

4.0.92 Drum - Universe ?

ner

$-78-9 \# 10 \# 11 \# 12 \quad$ imp:n-1 u=2 s interior

$2-392-1314-15(10 \cdot-11 \cdot 12) \quad$ imp:n=1 u=2 s Drum

Drum - Universe 3

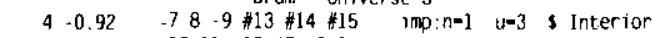

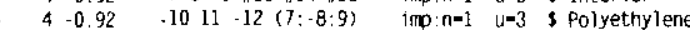

iner

$37 \quad 3 \cdot 0.0012913:-14: 15 \quad$ imp:n=1 $u=3$ s Air around Drum

Druan - Universe 4

$38 \quad 4-0.92 \quad-7$ B -9 \#16 \#17 \#18 imp:n-1 u=4 s Interior

$39 \quad 4-0.92 \quad .1011-12(7:-8: 9)$ imp:n-1 $u=4$ s Polyethylene

40 2 $-3.92-1314-15(10:-11: 12)$ ime:n=1 u=4 s Drum

$413 \cdot 0.00129$ 13:-14:15 imp:n=1 u=4 sir around Drumc

c $\quad$ Orum - Universe 5

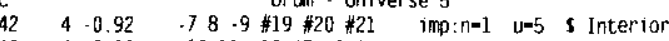

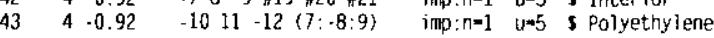

$44 \quad 2-3.92-1314-15(10:-11: 12)$ imp:n-1 u=5 s Drum

$453-0.0012913:-14: 15$ imo:n=1 $\mathrm{u}=5$ s Air around Drum

c Drum - Universe 6

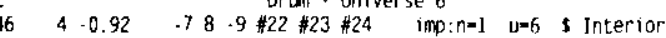

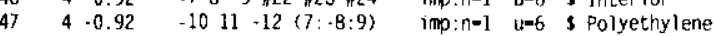

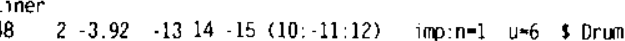

$493-0.0012913:-14: 15$ imp:n-1 $u=6$ s Air around Drum

C Drum. Universe 7

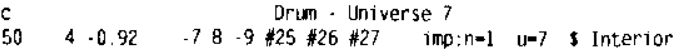

$51 \quad 4-0.92 \quad .1011-12(7:-8: 9) \quad i m p: n=1 \quad u=7$ s Polyethylene

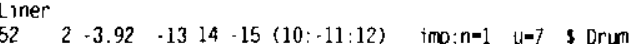

$53 \quad 3-0.00129$ 13:-14:15 imp:n=1 $u=7$, Air around Drumc

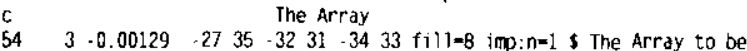
filled

$3 \cdot 0.00129$

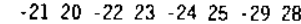

ued lat-2 imp:n=1 \$ Hex around Drum fi1 $1=-15: 15 \quad-15: 15 \quad-1: 2$

$2=-1$

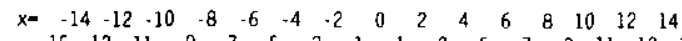
$\begin{array}{llllllllllllllll}15 & -13 & -11 & -9 & -7 & -5 & -3 & -1 & 1 & 3 & 5 & 7 & 9 & 11 & 13 & 15\end{array}$

$y=\cdot 15$

8888888888888888888888888888888 s

$y=-14$

$y=-13$

B8888888888888888888888888888885

$y=-12$

$y=-11$

$y=-10$

$y=-9$

$y=-8$

$y=-8$
$y=-7$

888 8 888888889888898888888888888

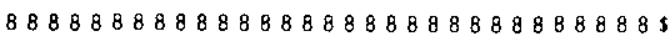

8888888888888888888888888888888 s

8888888888888888888868888888888 s

$888888 B 888888888888888888886888$ s $y=-6$

$y=\cdot 5$

$y=-4$

$y=-3$

$y=-2$

$y=\cdot 1$

$y=0$

$y=1$

$y=2$

$y=3$

$y=4$

$y=5$

$y=6$

$y=7$

$y=8$

$y=9$

$y=10$

$y=11$

$y-12$

$y=13$

$y=14$

$y=15$

$c$

c

c

c

$y=-15$

$y=-14$

$y=-13$

$y=-12$

$y=-11$

$y=-10$

$y=-9$

$y=-8$

$y=-7$

$y=-6$

$y=-5$

$y=-4$

$y=-3$

$y=-2$

$y=-1$

$y=0$

$y=1$

$y=2$

$y=3$

$y=4$
8888888888888888888888888888888

$8888888 B 8888888 B 888888888888 B 885$ $888888888888888888888888 B 8888885$ $88888 B 8888 B 88888888888868888888$ s

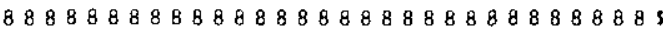
8 $8888888 B 8888888888888888888888$ s 8888888688888888888688888888888 s 888888888888888888888888 B88888Bs

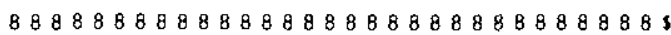
$88868888888888888888888888888 B 8$ 88888888888888888888888888888885 $88 B 88888888888888888 B 8888 B 88888$ $888888 B 8888888888888898888888 B 8$

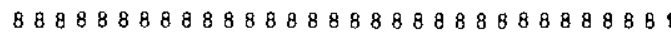
8888888888888888888888688888 888 $88 B 8888888888688888$ B88888888888s

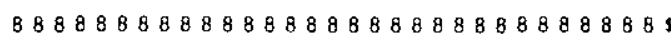
888888888888888888888 B888888888s 8888888888888888888868888888888 s $888888 B 888888888848888888888888$ s 8888888888888888888888888888888 8888888888888868888888888888888

$z=0$

$x=\begin{array}{lllllllllllllll}-14 & -12 & -10 & -8 & -6 & -4 & -2 & 0 & 2 & 4 & 6 & 8 & 10 & 12 & 14\end{array}$ $\begin{array}{llllllllllllllll}15 & -13 & -11 & -9 & -7 & -5 & -3 & -1 & 1 & 3 & 5 & 7 & 9 & 11 & 13 & 15\end{array}$ 8 888888888888888888888888888888 , 88888888888888888688888888888 B s

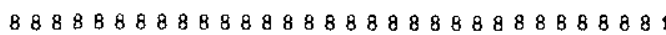
$888 B 888888888888888888888688888$,

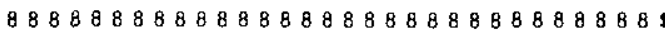
8888888888882342388888888888888 s 8886888888884234288888888888888 s $8888888888823423423423423488 B 88$ s 8888888888342342342342342388 B88 8888888888234234234234234288888 s 888888888842342342342342348888 Bs 8888888888342342342342342388888 s $88868888 B 4234234234234234288888$ s $88888888834234234234234234888 B 8$ s B88888888234234234234288888B888s 8888888884234234234234888888888 s $8888883423423423423423888898 B 88$ s 8 B888823423423423423428888888885 88888842342342342342388888888889 88888834234234234234288888888 8 s 
$y=5$

$y=6$

$y=7$

$y=8$

$y=9$

$y=10$

$y=11$

$y=12$

$y=13$

$y=14$

$y-15$

C

C

$\begin{array}{lllllllllllllllll}c & x= & -14 & -12 & -10 & -8 & -6 & -4 & -2 & 0 & 2 & 4 & 6 & 8 & 10 & 12 & 14\end{array}$

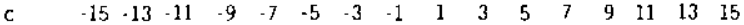

$y=-15$

$y=\cdot 14$

$y=-13$

$y=12$

$y=\cdot 11$

$y=-10$

$y=-9$

$y=-8$

$y=7$

$y=-6$

$y=-5$

$y=.4$

$y=-3$

$y=-2$

$y=-1$

$y=0$

$y=1$

$y=2$

$y=3$

$y=4$

$y=5$

$y=6$

$y=7$

$y-8$

$y=9$

$y=10$

$y=11$

$y=12$

$y=13$

$y=14$

$y=15$

88888823423423423423488888888865 8888884234234234234238888888888 s 8888883423423423423428888888888 s 8888882342342342342348888886888 s 8888884234234232888886888888888 s 8 888883423423423888888888888888 s 88888823423423428888888888888865

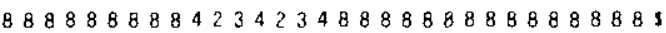
$88888 B 88834234238888 B 8 B 8 B 888 B B B 5$ 8888888882342342888888888888888 s 8888888884234234888888888888868 s

\section{$2=$} 888888888888888888888888888888 s $8888888888888 B 88888888888888888 \mathrm{~s}$

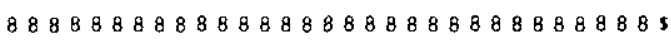
$888888888 B 888888888888886888888$ s 8888888888888888888888888886888 s $8888888888888888888888888888 B 88$ s 88 8888888B8888888888888888888B8s 8888888888888888888888888886888 s

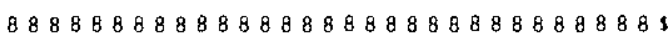
88888 8 $888888888888 B 888888888888$ s $8888 B 88888 B 88888888888888888886$ s 888888888888888888888888888888 s $8888888888888888888888 B 88888888$ s 8888888888888888888888888888888 s $88 B 88888888888$ B8888888888888888s $888888888888888888888888888888 \mathrm{~B}$ s

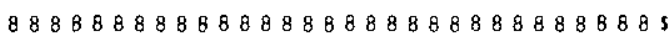
$88888888888888888 B 8888888 B 8888$ s 8888688888888888888888888888888 s 8888888888888888888888888888888 s

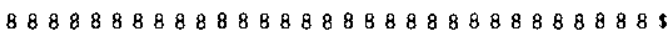
8888888888888888888888886888888 s B B88888888888888888888888888B885 8888688888888888888888888888888 s 8888888888888888888888888888888 s $8886888898 B 88888888886888$ B8888Bs B BBB8888BB8888BB88BBBBB8BBBBBBBS 8 888888888688888888886888888888 s

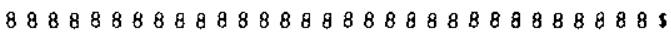
8888888888888888868888888888888 s

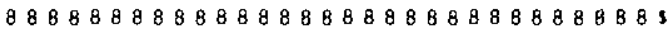
$c$

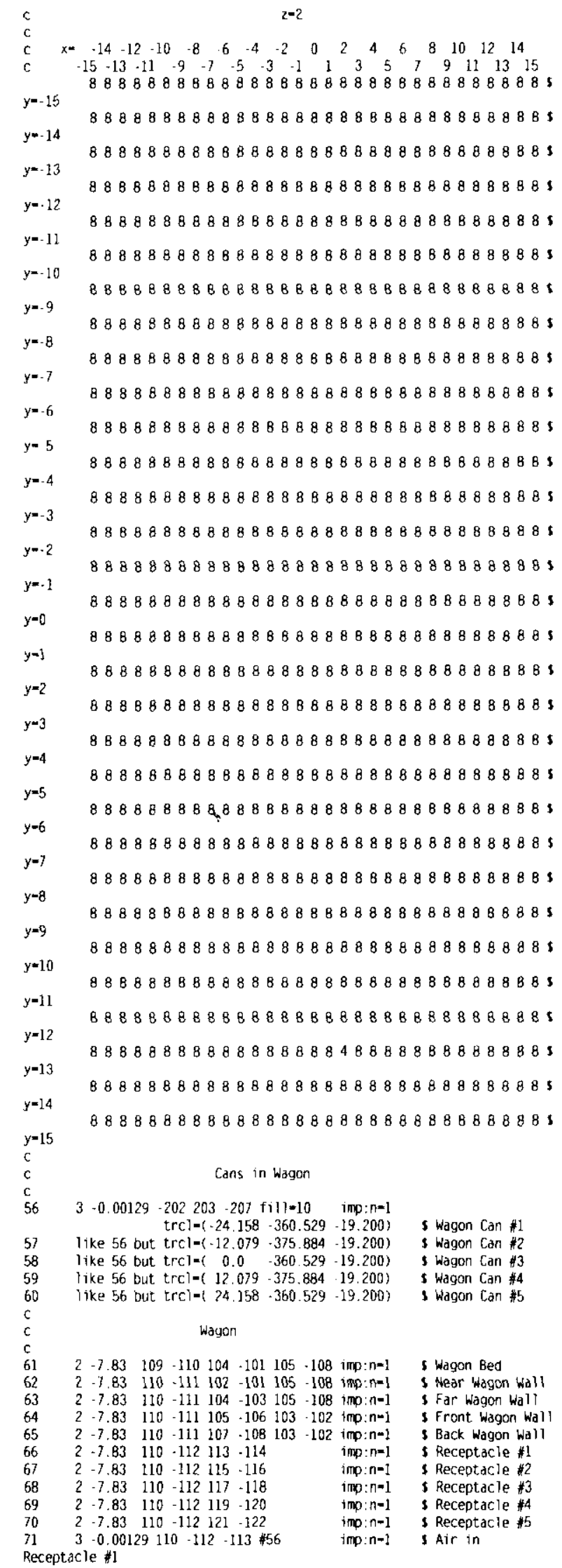

C-44 
HNF-6179 Rev. 0

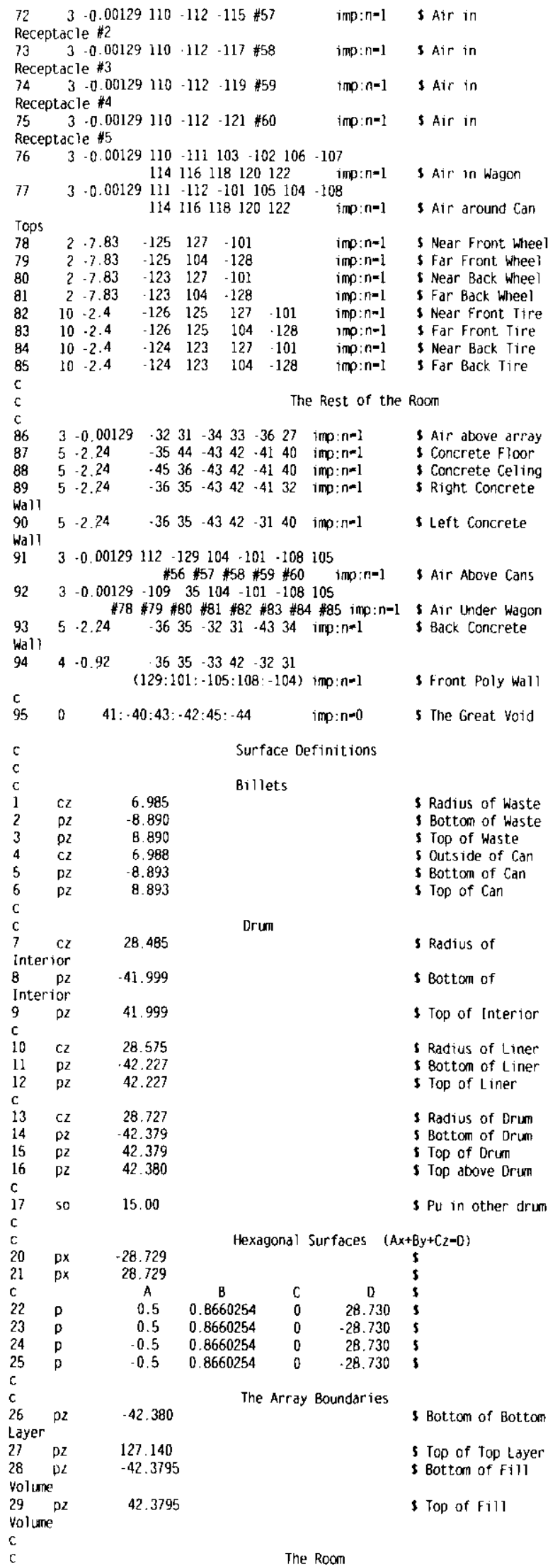

\begin{tabular}{|c|c|c|c|c|c|}
\hline $\begin{array}{l}31 \\
\text { Roan }\end{array}$ & $p x$ & \multicolumn{2}{|c|}{.350 .00} & & $s$ Left side of \\
\hline $\begin{array}{l}32 \\
\text { Room }\end{array}$ & $p x$ & \multicolumn{2}{|c|}{350.00} & & s Right. Side of \\
\hline 33 & py & \multicolumn{2}{|c|}{-351.50} & & s Front of Room \\
\hline 34 & py & \multicolumn{2}{|c|}{350.00} & & s Back of Room \\
\hline 35 & $\mathrm{pz}$ & \multirow{2}{*}{\multicolumn{2}{|c|}{$\begin{array}{l}-42.88 \\
150.00\end{array}$}} & & I floor of Room \\
\hline 36 & $p l$ & & & & S Celing of Room \\
\hline \multirow{2}{*}{\multicolumn{6}{|c|}{ Walls Coling Flon }} \\
\hline$c$ & & & & \multicolumn{2}{|c|}{ walls, Celing. Floor } \\
\hline 40 & $p x$ & \multicolumn{2}{|c|}{-380.48} & & s Left wall \\
\hline 41 & $p x$ & \multirow{2}{*}{\multicolumn{2}{|c|}{$\begin{array}{r}380.48 \\
-500.00\end{array}$}} & & s Right wall \\
\hline 42 & py & & & & S Front wall \\
\hline 43 & py & \multicolumn{2}{|c|}{380.48} & & S Back Wall \\
\hline 44 & $p 2$ & \multirow{3}{*}{\multicolumn{2}{|c|}{$\begin{array}{r}-100.00 \\
200.00\end{array}$}} & & s Floor \\
\hline 45 & $\mathrm{pz}$ & & & & s Celing \\
\hline \multirow{2}{*}{\multicolumn{3}{|c|}{$\begin{array}{l}c \\
c\end{array}$}} & & & \\
\hline & & & \multicolumn{2}{|l|}{ Wagon } & \\
\hline c & & & & & \\
\hline $\begin{array}{l}101 \\
\text { Wall }\end{array}$ & py & -352.050 & & & s Outside of Near Wagon \\
\hline $\begin{array}{l}102 \\
\text { Wall }\end{array}$ & py & -352.263 & & & S Inside of Near Wagon \\
\hline $\begin{array}{l}103 \\
\text { Wall }\end{array}$ & py & -383.887 & & & s Inside of Far Wagon \\
\hline $\begin{array}{l}104 \\
\text { Wall }\end{array}$ & py & -384.150 & & & s Outside of Far Wagon \\
\hline $\begin{array}{l}105 \\
\text { Wall }\end{array}$ & $p x$ & -35.430 & & & s Outside of front Wagon \\
\hline $\begin{array}{l}106 \\
\text { Wall }\end{array}$ & $p x$ & -35.167 & & & S Inside of Front Wagon \\
\hline $\begin{array}{l}107 \\
\text { wall }\end{array}$ & $p x$ & 35.167 & & & s Inside of Back Wagon \\
\hline $\begin{array}{l}108 \\
\text { Wall }\end{array}$ & $p x$ & 35.430 & & & s Outside of Back wagon \\
\hline 109 & p2 & -19.521 & & & 5 Botton of wagon \\
\hline 110 & D2 & -19.258 & & & S Floor of Wagon \\
\hline 111 & pz & -6.558 & & & s Top of Wagon Walls \\
\hline 112 & pz & .4 .018 & & & s Top of Receptacle \\
\hline c & & $x$ & y & $R$ & \\
\hline $\begin{array}{l}113 \\
\text { Radius }\end{array}$ & $\mathrm{c} / 2$ & -24.158 & .360 .529 & 6.724 & s Receptacle \#1 liner \\
\hline $\begin{array}{l}114 \\
\text { Radius }\end{array}$ & $c / 2$ & -24.158 & .360 .529 & 7.065 & I Receptacle \#1 Outer \\
\hline $\begin{array}{l}115 \\
\text { Radius }\end{array}$ & $\mathrm{C} / 2$ & -12.079 & -375.884 & 6.724 & S Receptacle \#2 Inner \\
\hline $\begin{array}{l}116 \\
\text { Radius }\end{array}$ & $\mathrm{c} / \mathrm{z}$ & -12.079 & -375.884 & 7.065 & s Receptacle \#2 Outer \\
\hline $\begin{array}{l}117 \\
\text { Radius }\end{array}$ & $c / 2$ & 0.000 & -360.529 & 6.724 & S Receptacle $\# 3$ Inner \\
\hline $\begin{array}{l}118 \\
\text { Radius }\end{array}$ & $\mathrm{c} / 2$ & 0.000 & -360.529 & 7.065 & 5 Receptacle $\$ 3$ Outer \\
\hline $\begin{array}{l}119 \\
\text { Radius }\end{array}$ & $c / 2$ & 12.079 & -375.884 & 6.725 & Seceptacie \#4 Inner \\
\hline $\begin{array}{l}120 \\
\text { Radius }\end{array}$ & $\mathrm{c} / 2$ & 12.079 & -375.884 & 7.065 & S Receptacle \#4 Outer \\
\hline $\begin{array}{l}121 \\
\text { Radius }\end{array}$ & $\mathrm{C} / \mathrm{z}$ & 24.158 & .360 .529 & 6.725 & S Receptacle \#5 Inner \\
\hline $\begin{array}{l}122 \\
\text { Radius }\end{array}$ & $\mathrm{c} / 2$ & 24.158 & -360.529 & 7.065 & \$ Receptacle $\$ 5$ Outer \\
\hline$c$ & & $\mathrm{x}$ & z & $\mathrm{R}$ & \\
\hline 123 & $c / y$ & 23.980 & -30.951 & 10.420 & S Back whee I Radius \\
\hline 124 & $\mathrm{c} / \mathrm{y}$ & 23.980 & -30.951 & 11.420 & s Back fire Radius \\
\hline 125 & $\mathrm{c} / \mathrm{y}$ & -23.980 & -30.951 & 10.420 & S Front wheel Radius \\
\hline 126 & $\mathrm{c} / \mathrm{y}$ & -23.980 & .30 .951 & 11.420 & s Front Tire Radius \\
\hline$c$ & & & & & \\
\hline 127 & py & .353 .260 & & & S Inside of Near wheels \\
\hline 128 & py & -382.940 & & & S Inside of Far wheels \\
\hline 129 & $\mathrm{pz}$ & 10.000 & & & 5 Top of Air Above Wagon \\
\hline
\end{tabular}

- Inside of Wagon Can

5 Outside of Wagon Can

5 Bottom of Wagon Can

3 Botton of Plutoniun

3 Top of Plutonium

$\checkmark$ Inside of Top of wagon

$s$ Outside of Top of

5 Radius of Plutoniuan 
HNF-6179 Rev. 0

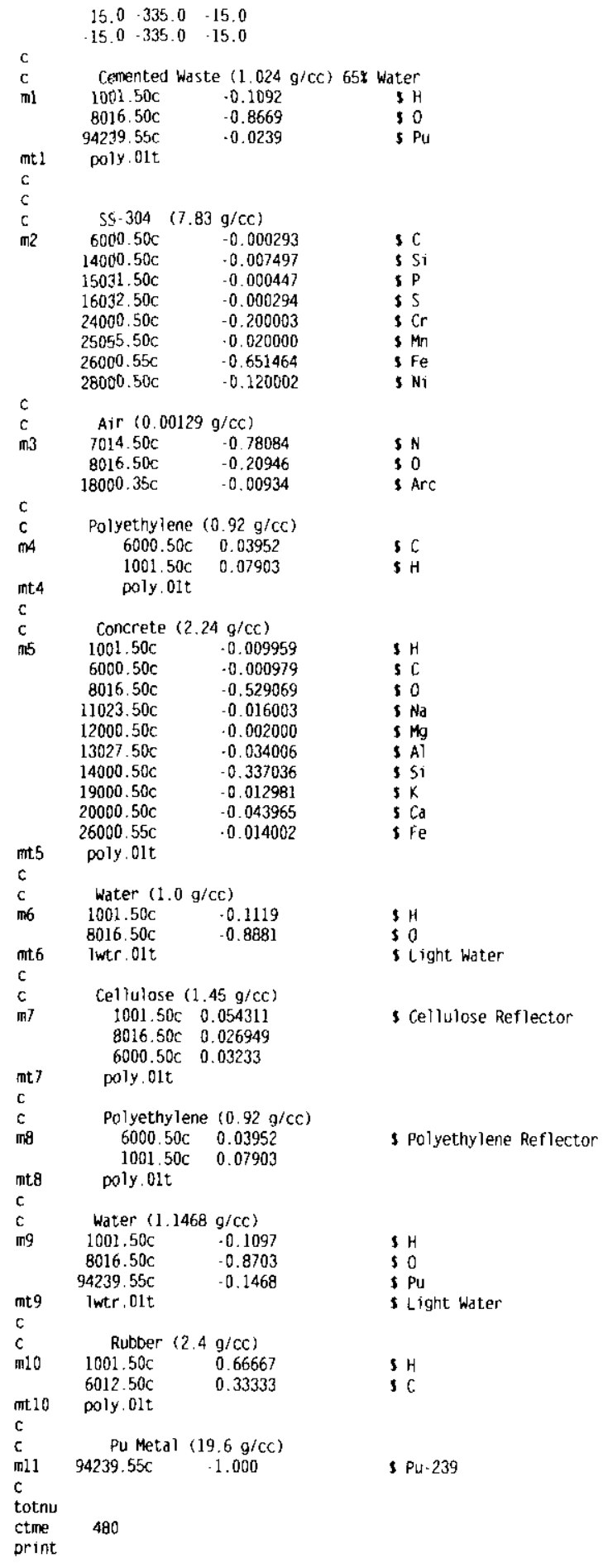

nonplpe_ref21, Inp

Three Billets in a Non-pipe 55 Gallon Drum

three $66.7 \mathrm{~g} \mathrm{Pu}$ billets at the bottom of a $200 \mathrm{~g} / \mathrm{drum}$

Infinite relection. Two Layers

silicon replaces Calcium

Cell Definitions

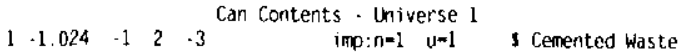

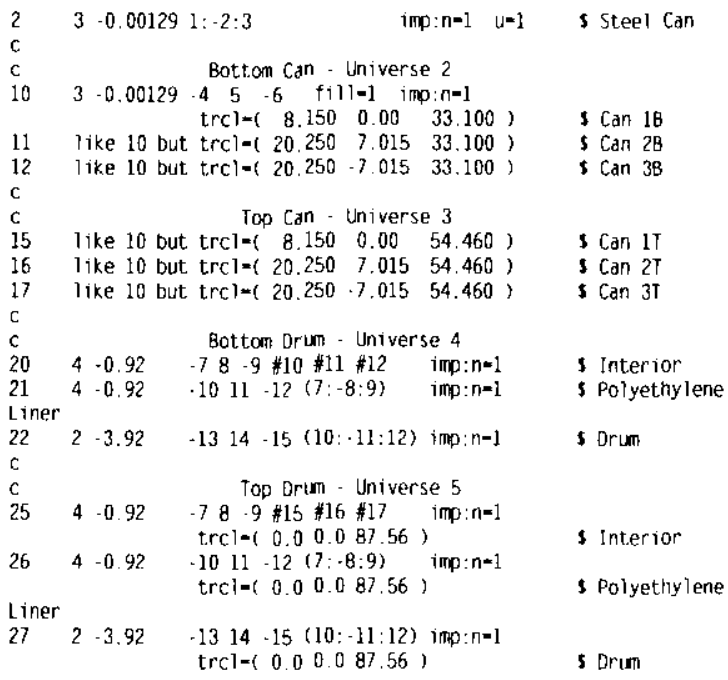

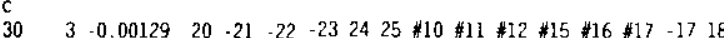

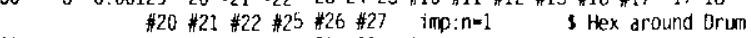

$\begin{array}{llllllllllll}31 & 5 & -2 & 24 & 38 & -18 & 20 & -21 & \cdot 22 & -23 & 24 & 25\end{array}$

$\begin{array}{lllllllllll}32 & 3 & -0.00129 & -37 & 17 & 20 & -21 & -22 & -23 & 24 & 25\end{array}$

imp:n=1 S Air ADove Drums

$500 \quad(-20: 21: 22: 23:-24:-25: 37:-38)$ imo:n-0 S The Great void

Surface Definitions

Billets

Radius of waste

Botton of Waste

5 Top of Waste

s Outside of Can

Bottom of Can

s Top of Can

5 Radius of

S Botton of

5 Top of interior

S Radius of Liner sottom of Liner s Top of Liner

S Radius of Drum Botton of Brum Top of Orum 3 Top above Drum s Top above Top

s floor

$-42.380$

Hexagonal Surfaces $(A x+B y+C z=D)$

$\begin{array}{llr}* 20 & \text { px } & -28.729 \\ \star 21 & \text { px } & 28.729\end{array}$

$\begin{array}{llcccc}C & & A & B & C & D \\ \star 22 & D & 0.5 & 0.8660254 & 0 & 28.730 \\ * 23 & D & -0.5 & 0.8660254 & 0 & 28.730 \\ \star 24 & D & 0.5 & 0.8660254 & 0 & -28.730\end{array}$

$\begin{array}{llcccc}C & & A & B & C & D \\ \star 22 & D & 0.5 & 0.8660254 & 0 & 28.730 \\ * 23 & D & -0.5 & 0.8660254 & 0 & 28.730 \\ \star 24 & D & 0.5 & 0.8660254 & 0 & -28.730\end{array}$

$\begin{array}{rrrrr}0.5 & 0.8660254 & 0 & -28.730 & \$ \\ -0.5 & 0.8660254 & 0 & -28.730\end{array}$

$\begin{array}{llll}\mathrm{C} & & & \\ 36 & \mathrm{pz} & -42.381 & \text { s Concrete Floor }\end{array}$

$\begin{array}{llll}36 & p 2 & -42.381 & \text { s Concrete Floor } \\ 37 & p 2 & 200.00 & \text { s Top of the World } \\ 38 & \text { pz } & -100.00 & \text { s Bottom of the }\end{array}$

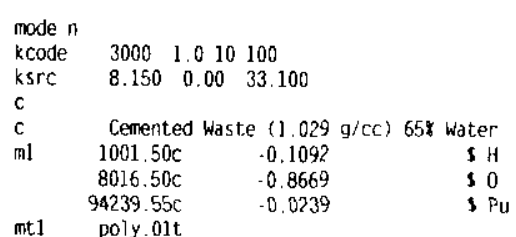

mit poly.01t 
HNF-6179 Rev. 0

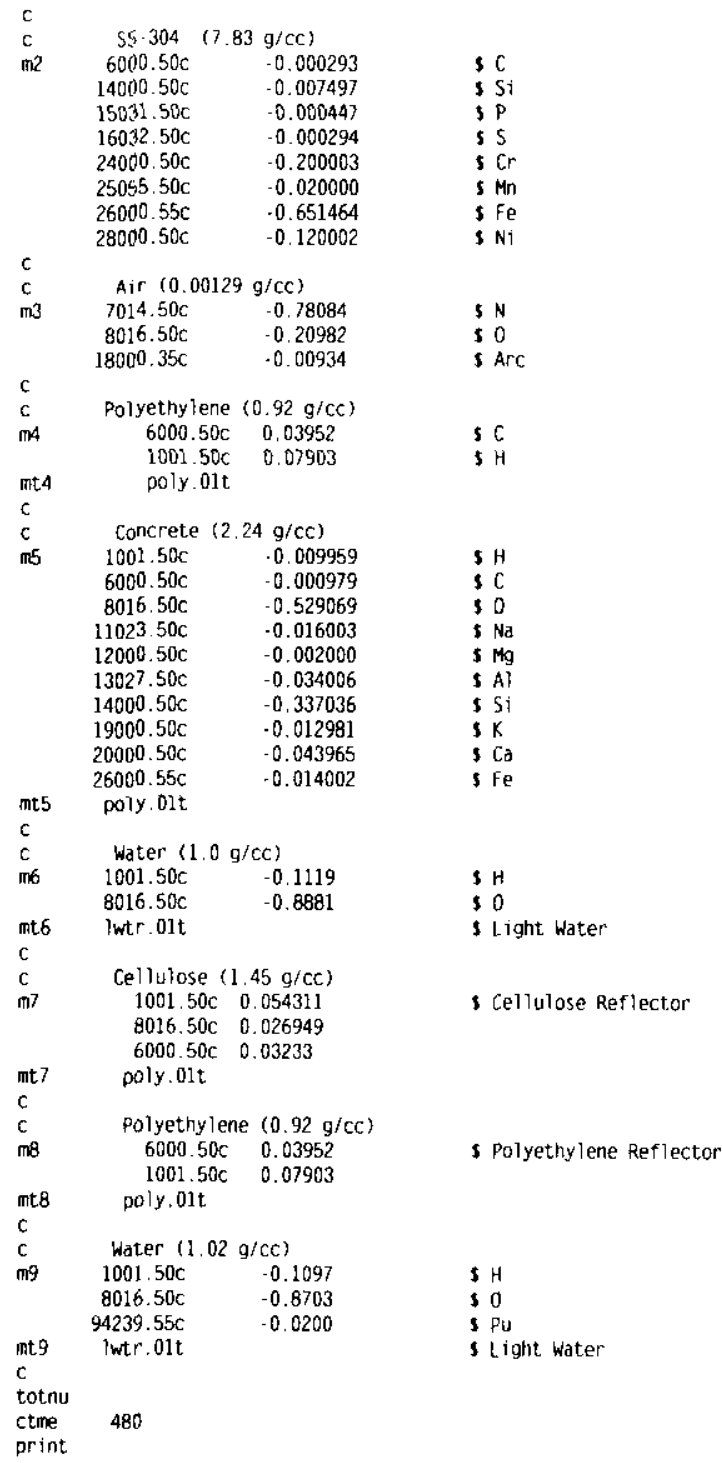

nonpipe.ref2p.inp

Three Billets in a Non-pipe 55 Galion Orum

$c \quad$ three $66.7 \mathrm{~g}$ Pu billets at the bottom of a $200 \mathrm{~g} / \mathrm{drum}$

one relecting boundary (two drums)-poly

Cell Definitions

$\begin{array}{lllllll}1 & -1.024 & -1 & 2 & -3 & \text { Can Contents - Universe } 1 \\ \text { imp:n=1 } u=1\end{array}$

$\begin{array}{llllllll}1 & -1.024 & -1 & 2 & -3 & \text { imp: } n=1 & u=1 \\ 3 & -0.00129 & 1:-2: 3 & \text { s Cemented waste }\end{array}$

$3.0 .00129-4 \quad 5 \quad-6 \quad$ Can - Universe 2 $.45 \quad-6 \quad$ fill $=1$ imp: $n=1$

like 10 but trcl- $(20.250 \quad 7.015-33.100$ s Can 1

11 like 10 but $\operatorname{trcl}-\left(\begin{array}{rrrr}20.250 & 7.015 & -33.100) & \text { \& Can } 2 \\ 12 & \text { like } 10 \text { but } \operatorname{trcl}-(20.250 & -7.015 & -33.100\end{array}\right)$ s Can 3

c $\quad$ Orum - Universe 2

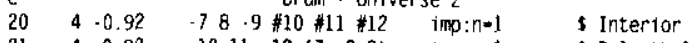

$21 \quad 4-0.92 \quad-1011-12(7:-8: 9) \quad$ imp:n-1 s Polyethylene

Liner

$24 \quad 2-3.92 \quad-1314-15(10:-11: 12)$ imp: $n=1 \quad$ s or um

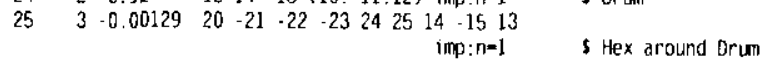
c The Room

$30 \quad 3-0.00129 \quad-3736(-20: 21: 22: 23:-24:-25: 15:-14) \cdot 21$

\begin{tabular}{|c|c|c|c|c|c|c|}
\hline \multirow{2}{*}{$\begin{array}{l}31 \\
c \\
50\end{array}$} & $5-2.2$ & $-36-3$ & $37-21$ & \multicolumn{2}{|r|}{$\begin{array}{l}i m p: n=1 \\
i m p: n=1\end{array}$} & $\begin{array}{l}\text { S Air around Drum } \\
\text { S Concrete Fioor }\end{array}$ \\
\hline & 0 & $37: 21$ & & & $\operatorname{imp}: n=0$ & s The Great void \\
\hline c & \multirow{2}{*}{\multicolumn{6}{|c|}{ Surface Definitions }} \\
\hline $\begin{array}{l}c \\
c\end{array}$ & & & & & & \\
\hline 1 & $c 2$ & 6.985 & Dim & & & \$ Radius of Waste \\
\hline 2 & $p 2$ & 8.890 & & & & s Botton of waste \\
\hline 3 & $p z$ & 8.890 & & & & S Top of Waste \\
\hline 4 & $c z$ & 6.988 & & & & S Outside of Can \\
\hline 5 & $\mathrm{pz}$ & -8.893 & & & & s Bottom of Can \\
\hline 6 & $p 2$ & 8.893 & & & & $s$ Top of Can \\
\hline $\begin{array}{l}c \\
c\end{array}$ & \multirow{2}{*}{\multicolumn{5}{|c|}{ Drum }} & \\
\hline $\begin{array}{l}\mathrm{C} \\
7\end{array}$ & & & & & & \\
\hline \multicolumn{7}{|c|}{ Inter or } \\
\hline B & $p z$ & -41.999 & & & & s Bottom of \\
\hline \multicolumn{7}{|c|}{ Interior } \\
\hline 9 & $p z$ & 41.999 & & & & s Top of Interior \\
\hline \multicolumn{7}{|r|}{-5} \\
\hline 10 & $\mathrm{Cz}$ & 28.575 & & & & $\$$ Radius of Liner \\
\hline 11 & $\begin{array}{l}p z \\
p z\end{array}$ & $\begin{array}{r}-42.227 \\
42.227\end{array}$ & & & & $\begin{array}{l}\text { Bottom of Liner } \\
\text { Top of Liner }\end{array}$ \\
\hline \multicolumn{7}{|r|}{ 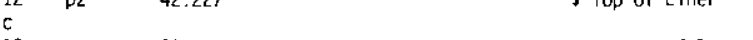 } \\
\hline 13 & $c z$ & 28.727 & & & & 5 Radius of Drum \\
\hline 14 & pz & .42 .379 & & & & s Botton of Drum \\
\hline 15 & $p z$ & 42.379 & & & & \$ Top of Drum \\
\hline 16 & $p z$ & 42.380 & & & & s Top above Drum \\
\hline \multirow{2}{*}{\multicolumn{7}{|c|}{ Hexagonal Surfaces }} \\
\hline & & & & & & $(+B y+(z-0)$ \\
\hline $\begin{array}{l}20 \\
\star 21\end{array}$ & $p x$ & -28.729 & & & & 3 \\
\hline $\begin{array}{l}\star^{\star} 21 \\
c\end{array}$ & $p x$ & 28.729 & & & & 3 \\
\hline $\begin{array}{l}c \\
22\end{array}$ & & A & B & $c$ & D & 5 \\
\hline $\begin{array}{l}22 \\
23\end{array}$ & $\begin{array}{l}p \\
0\end{array}$ & $\begin{array}{r}0.5 \\
-0.5\end{array}$ & 0.8660254 & 0 & 28.730 & $\begin{array}{l}5 \\
5\end{array}$ \\
\hline 24 & p & 0.5 & 0.8660254 & 0 & $\begin{array}{r}28.730 \\
-28.730\end{array}$ & 5 \\
\hline 25 & $p$ & -0.5 & 0.8660254 & 0 & -28.730 & 5 \\
\hline $\begin{array}{l}C \\
36\end{array}$ & $p z$ & -42.381 & & & & s Concrete Floor \\
\hline 37 & so & 100.00 & & & & S End of the world \\
\hline
\end{tabular}

$3000 \quad 1.010100$

$\begin{array}{lllll}\text { ksre } & 8.150 & 0.00 & -33.100\end{array}$

c Cenented waste (1.024 g/cc) 65\% Water

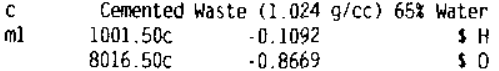

$\begin{array}{rrr}8016.50 \mathrm{c} & -0.8669 & \text { s. } 0 \\ 94239.55 \mathrm{C} & -0.0239 & \text { s } \mathrm{Pu}\end{array}$

mt1 poly $01 \mathrm{t}$

c $\quad 55.304 \quad(7.83 \mathrm{~g} / \mathrm{CC})$

$\begin{array}{ccc}55.304 & (7.83 \mathrm{~g} / \mathrm{CC}) & \\ 6000.50 \mathrm{C} & -0.000293 & \text { SC }\end{array}$

$\begin{array}{lll}14000.50 \mathrm{C} & -0.007497 & 5 \mathrm{~S} \\ 15031.50 \mathrm{C} & -0.000447 & \mathrm{~s}\end{array}$

$16032.50 \mathrm{C}-0.000294$ s

$24000.50 \mathrm{C} \quad 0.200003 \quad \mathrm{~S} \mathrm{Cr}$

$25055.50 \mathrm{C}-0.020000 \quad$ s in

$26000.55 \mathrm{C} \quad-0.651464 \quad$ S Fe

$28000.50 \mathrm{C}-0.120002$ I $\mathrm{Ni}$

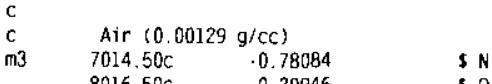

$8016.50 \mathrm{C}-0.20946 \quad$ \$ 0

c Polyethylene $(0.92 \mathrm{~g} / \mathrm{cc})$

$\begin{array}{lll}6000.50 \mathrm{c} & 0.03952 & \text { S C } \\ 1001.50 \mathrm{c} & 0.07903 & \text { S H }\end{array}$

mt4 poly.01t

(1)

C Concrete $(2.24 \mathrm{~g} / \mathrm{cc})$

m5 $1001.50 \mathrm{C} \quad-0.009959$

$\begin{array}{lll}1001.50 \mathrm{C} & -0.009959 & \text { S H } \\ 6000.50 \mathrm{C} & -0.000979 & \text { S C }\end{array}$

$\begin{array}{lll}6000.50 \mathrm{C} & -0.000979 & 5 \mathrm{C} \\ 8016.50 \mathrm{C} & -0.529069 & \text { 5 } 0\end{array}$

$11023.50 \mathrm{c}-0.016003 \quad$ is

$12000.50 \mathrm{C} \quad-0.002000 \quad 5 \mathrm{Mg}$

$14000.50 \mathrm{C}-0.337036$ S $5 \mathrm{si}$

$\begin{array}{lll}19000.50 \mathrm{C} & -0.012981 & \text { S K } \\ 20000.50 \mathrm{C} & -0.043965 & \text { S Ca }\end{array}$

$\begin{array}{lll}20000.50 \mathrm{C} & -0.043965 & \text { S } \mathrm{Ca} \\ 26000.55 \mathrm{C} & -0.014002 & \text { S Fe }\end{array}$

mit5 poly.01t

c water $(1.0 \mathrm{~g} / \mathrm{cc})$

m6 $\quad 1001.50 \mathrm{C} r-0.1119 \quad 5 \mathrm{H}$

$\begin{array}{lll}1001.50 \mathrm{C} & -0.1119 & 5 \mathrm{H} \\ 8016.50 \mathrm{C} & -0.8881 & \$ \mathrm{O}\end{array}$ 
HNF-6179 Rev. 0

\begin{tabular}{|c|c|c|}
\hline mt6 & lwt.r.01t & s Light water \\
\hline c & C:ollulose $(1.45 \mathrm{~g} / \mathrm{cc})$ & \\
\hline my & $\begin{array}{ll}1001.50 \mathrm{C} & 0.054311 \\
8016.50 \mathrm{c} & 0.026949 \\
6000.50 \mathrm{c} & 0.03233\end{array}$ & \$ Cellulose Reflector \\
\hline $\begin{array}{l}\text { nt } 7 \\
\text { c }\end{array}$ & poly.01t & \\
\hline $\begin{array}{l}c \\
\mathrm{~m} 8\end{array}$ & $\begin{array}{cc}\text { Polyethylene } & (0.92 \mathrm{~g} / \mathrm{CC}) \\
6000.50 \mathrm{C} & 0.03952 \\
1001.50 \mathrm{C} & 0.07903\end{array}$ & s Polyethylene Reflector \\
\hline $\begin{array}{l}\mathrm{mt8} \\
\mathrm{c}\end{array}$ & poly olt & \\
\hline $\begin{array}{c}c \\
m 9\end{array}$ & $\begin{array}{cc}\text { Water }(1.02 \mathrm{~g} / \mathrm{cc}) \\
1001.50 \mathrm{C} & -0.1097 \\
8016.50 \mathrm{C} & -0.8703 \\
94239.55 \mathrm{C} & -0.0200\end{array}$ & $\begin{array}{l}\text { S } \mathrm{H} \\
\$ 0 \\
\$ \mathrm{Pu}\end{array}$ \\
\hline $\begin{array}{l}\mathrm{mt} 9 \\
\mathrm{c} \\
\text { totnu }\end{array}$ & lwtr.01t & s Light water \\
\hline $\begin{array}{l}\text { ctme } \\
\text { print }\end{array}$ & 480 & \\
\hline
\end{tabular}

nonpípe. refp. inp

Three Billets in a Non-pipe 55 Gallon Drum

c three $66.7 \mathrm{~g}$ Pu billets at the bottom of a $200 \mathrm{~g} / \mathrm{drum}$ infinite reflection-Poly

\section{Cell Definitions}

$\begin{array}{llllll}1+1.024 & -1 & 2 & -3 & \text { Can Contents - Universe } 1 \\ \text { imp: } n=1 \quad u=1\end{array}$ $\begin{array}{llllll}1-1.024-1 & 2-3 & \text { imp:n-1 } u=1 & \text { s Cemented waste } \\ 3-0.00129 & 1:-2: 3 & \text { imp:n=1 } u=1 & \text { s Steel Can }\end{array}$

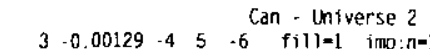
trcler $8.150 \quad 0.00-33.100)$

$11 \quad$ lixe 10 but $\operatorname{trc})=(20.250,7.015-33.100)$

$11 \quad$ like 10 but $\operatorname{trc})=\left(\begin{array}{ccc}20.250 & 7.015 & -33.100\end{array}\right)$

s Can 1

Orum - Universe 2

$\begin{array}{llllll}20 & 4 & -0.92 & -78-9 * 10 \# 11 * 12 & \text { imp:n-1 } & \text { \$ Interior } \\ 21 & 4 & -0.92 & -1011-12(7:-8: 9) & \text { imp:n-1 } & \text { s Polyethyl }\end{array}$

Liner

$24 \quad 2-3.92-1314-15(10:-11: 12)$ imp:n-1

$\begin{array}{lllllllllllll}25 & 3 & -0.00129 & 20 & -21 & -22 & -23 & 24 & 25 & 14 & -15 & 13\end{array}$

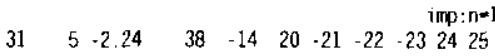

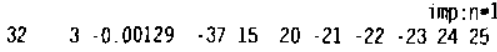

c $\quad(\cdot 20: 21: 22: 23:-24:-25 \cdot 37: 38)$ imp:n=0

Polyethylene

$\checkmark$ Drum

5 Hex around Drum

5 Concrete Floor

- Air Above Drums

s The Great void

\begin{tabular}{|c|c|c|c|c|c|c|}
\hline $\begin{array}{l}c \\
c\end{array}$ & & \multicolumn{5}{|c|}{ Surface Definitions } \\
\hline$c$ & & \multicolumn{4}{|c|}{ Billets } & \\
\hline 1 & $C Z$ & 6.985 & & & & S Radius of Waste \\
\hline 2 & $p z$ & .8 .890 & & & & s Bottom of Waste \\
\hline 3 & $p 2$ & 8.890 & & & & S Top of Waste \\
\hline 4 & $c z$ & 6.988 & & & & s Outside of Can \\
\hline 5 & $p z$ & .8 .893 & & & & $s$ Bottom of Can \\
\hline $\begin{array}{l}6 \\
c\end{array}$ & pz & 8.893 & & & & $s$ Top of Can \\
\hline 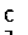 & & & Drum & & & \\
\hline In & $\begin{array}{c}\text { CZ } \\
\text { ior }\end{array}$ & 28.485 & & & & s Radius of \\
\hline 8 & $\rho 2$ & -41.999 & & & & $\$$ Bottom of \\
\hline Int & or & & & & & \\
\hline 9 & $p 2$ & 41.999 & & & & s Top of Interior \\
\hline 10 & $c z$ & 28.575 & & & & \\
\hline 11 & $p z$ & .42 .227 & & & & $\$$ Botton of Liner \\
\hline 12 & $p z$ & 42.227 & & & & S Top of Liner \\
\hline & & & & & & \\
\hline 13 & $C 2$ & 28.727 & & & & \$ Radius of Drum \\
\hline 14 & $p z$ & -42.379 & & & & Botton of Drum \\
\hline 15 & $\mathrm{p} 2$ & 42.379 & & & & S Top of Orum \\
\hline 16 & $p 2$ & 42.380 & & & & \$ Top above Drun \\
\hline & & & Hexagc & a) 5 & faces & $A x+B y+(z=-D)$ \\
\hline & $p x$ & -28.729 & & & & 5 \\
\hline 2 & $p x$ & 28.729 & & & & s \\
\hline & & A & 8 & c & D & s \\
\hline 22 & p & 0.5 & 0.8660254 & 0 & 28.730 & $\mathbf{s}$ \\
\hline 23 & $\mathrm{P}$ & -0.5 & 0.8660254 & 0 & 28.730 & s \\
\hline+2 & $p$ & 0.5 & 0.8660254 & 0 & .28 .730 & 5 \\
\hline$k^{2}$ & D & -0.5 & 0.8660254 & 0 & .28 .730 & s \\
\hline
\end{tabular}

$\begin{array}{llll}\text { C } & & -42.381 & \text { s Concrete Floor } \\ 36 & p z & 100.00 & \text { s Top of the Wor } 1\end{array}$

$\begin{array}{lll}38 \quad 02 & -100.00 & \text { s Bottom of the }\end{array}$

$\begin{array}{lllll}\text { mode } & & & & \\ \text { kcode } & 3000 & 1.0 & 10 & 100\end{array}$

$\begin{array}{llll}\text { ksrc } & 8.150 & 0.00 & -33.100\end{array}$

C Cenented waste $(1.024 \mathrm{~g} / \mathrm{cc}) 65 \%$ water

$\mathrm{m} 1 \mathrm{1001.50 \textrm {C }}-0.1092 \quad \mathrm{H}$ $\begin{array}{rrr}8016.50 \mathrm{C} & -0.8669 & 5 \mathrm{O} \\ 94239.55 \mathrm{C} & -0.0239 & \text { S Pu }\end{array}$

mt1 poly.01

c

c $\quad 5 S-304 \quad(7.83 \mathrm{~g} / \mathrm{CC})$

$6000.50 \mathrm{C} \quad-0.000293 \quad$ s

$16032.50 \mathrm{C}-0.00044$

$24000.50 \mathrm{c}-0.200003$

$25055.50 \mathrm{c}-0.20003$

26000.55

$28000.50 \mathrm{C} \quad-0.120002$

$5 \mathrm{Si}$
$5 \mathrm{P}$
$5 \mathrm{~S}$
$5 \mathrm{Cr}$
$5 \mathrm{Mr}$
$5 \mathrm{Fe}$
$\$ \mathrm{Ni}$

c $\quad$ Air $(0.00129 \mathrm{~g} / \mathrm{CC})$

$\begin{array}{lll}7014.50 \mathrm{C} & -0.78084 & 5 \mathrm{~N} \\ 8016.50 \mathrm{C} & -0.20982 & 50\end{array}$

$18000.35 \mathrm{C} \quad-0.00934$ S Ar

c Polyethylene $(0.92 \mathrm{~g} / \mathrm{cc})$

m4 $\begin{array}{lll}6000.50 \mathrm{c} & 0.03952 & \text { S } \\ 1001.50 \mathrm{c} & 0.07903 & \text { i }\end{array}$

mit4 poly.01t 0.07903 S

c Concrete $(2.24 \mathrm{~g} / \mathrm{cc})$

c Concrete $(2.24 \mathrm{~g} / \mathrm{cc})$

$\begin{array}{lll}1001.50 \mathrm{C} & -0.009959 & \text { \$ } \\ 6000.50 \mathrm{C} & -0.000979 & \text { s }\end{array}$

$\begin{array}{lll}6000.50 \mathrm{C} & -0.000979 & \text { S } \\ 8016.50 \mathrm{C} & -0.529069 & \text { s }\end{array}$

$11023.50 \mathrm{C}-0.016003 \quad$ S Na

$12000.50 \mathrm{C} \quad-0.002000 \quad$ s Mg

$\begin{array}{lll}13027.50 \mathrm{C} & 0.034006 & \text { S AF } \\ 14000.50 \mathrm{c} & -0.337036 & \text { s }\end{array}$

$19000.50 \mathrm{C}-0.012981$ \&

$20000.50 \mathrm{C}-0.043965$ s Ca

$\begin{array}{lll}20000.50 \mathrm{C} & -0.043965 & \text { s Ca } \\ 26000.55 \mathrm{C} & .0 .014002 & \text { s Fe }\end{array}$

mt5 poly.01t

$c$
$c$
int water $(1.0 \mathrm{~g} / \mathrm{cc})$

m6 $\begin{array}{ccc}1001.50 \mathrm{C} & -0.1119 & \text { s } \\ 8016.50 \mathrm{C} & -0.8881 & \text { s }\end{array}$

mt6 Iwtr.01t s Light Water

c Cellulose $(1.45 \mathrm{~g} / \mathrm{cc})$

$1001.50 \mathrm{C} \quad 0.05431$

$8016.50 \mathrm{C} \quad 0.026949$

$6000.50 \mathrm{C} \quad 0.03233$

mt7 poly.01t

c $\quad$ Polyethylene $(0.92 \mathrm{~g} / \mathrm{cc})$

$6000.50 \mathrm{C} \quad 0.03952$

$\begin{array}{cc}1001.50 \mathrm{C} & 0.07903 \\ \text { poly. } 01 \mathrm{t} & \end{array}$

s Cellulose Reflector

Water $(1.02 \mathrm{~g} / \mathrm{cc})$

$1001.50 \mathrm{C} \quad-0.1097$

$8016.50 \mathrm{C} \quad-0.8703$

$94239.55 \mathrm{C} \quad .0 .0200$

nt9 iwtr.01t

5 Pu

5 Light water

c

totnu

ctme $\quad 480$

print

nonp1pe_refrot21. Inp

Three Bitlets in a Non-pipe 55 Gallon Drum

three $66.7 \mathrm{~g}$ Pu billets at the bottom of a $200 \mathrm{~g} / \mathrm{drum}$

Infinite relection -Two Layers

silicon replaces Calcion

Cell Definitions

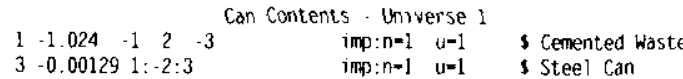


HNF-6179 Rev. 0
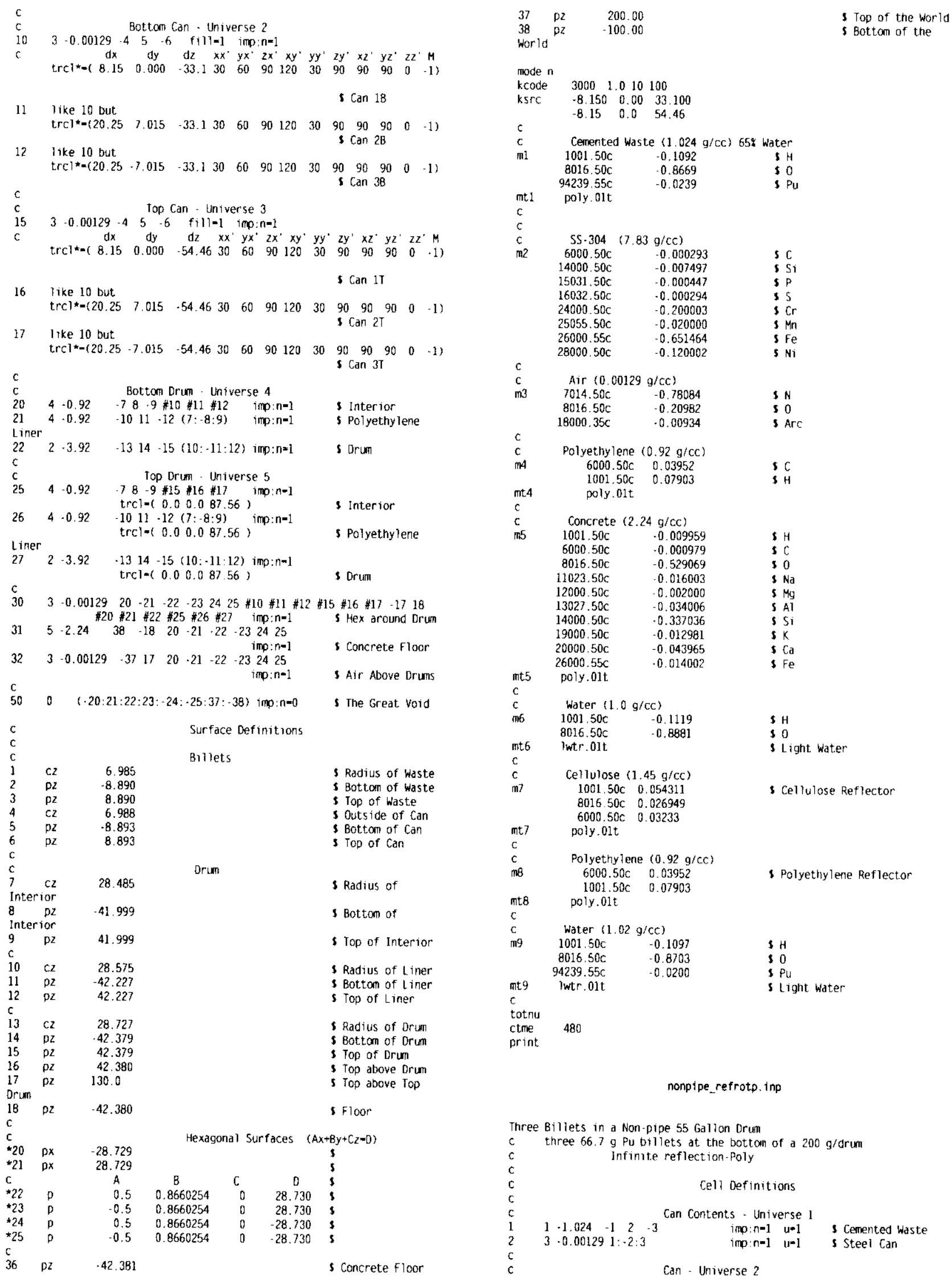


\section{HNF-6179 Rev. 0}

$10 \quad 3-0.00129-4 \quad 5 \quad-6 \quad$ fill-1 $\quad$ imo:n-1

$d x \quad d y \quad d z \quad x x^{\prime} y x^{\prime} z x^{\prime} x y^{\prime} y y^{\prime} z y^{\prime} x z^{\prime} y z^{\prime} z z^{\prime} M$

11 like 10 but

S Can 1

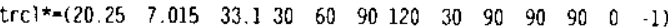

5 Can 2

12 like 10 but

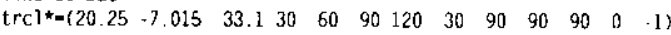

s Can 3

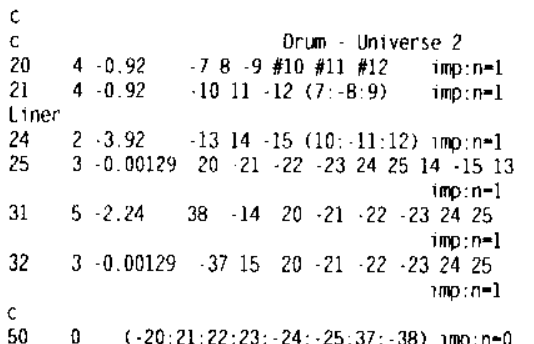

$500(-20: 21: 22: 23:-24:-25: 37:-38)$ 1mp:n-0

$\begin{array}{ll}C 2 & 6.985 \\ 02 & -8.890\end{array}$

pz $\quad 8.890$

CZ 6.98

$\begin{array}{lr}\mathrm{p} 2 & -8.893 \\ \mathrm{pz} & 8.893\end{array}$

cz

8.893

Surface Definitions

Billets

8 interior

$9 \quad$ Interior

$10 \quad \mathrm{CZ} \quad 28.575$

$\begin{array}{llr}11 & p z & -42.227 \\ 12 & p z & 42.227\end{array}$

$\begin{array}{lll}\mathrm{C} & & \\ 13 & \mathrm{Cl} & 28.727\end{array}$

$\begin{array}{llr}14 & 02 & -42.379 \\ 15 & 02 & 42.379\end{array}$

$\begin{array}{lll}15 & p 2 & 42.379 \\ 16 & \text { pz } & 42.380\end{array}$

c

c

*20 px

$\star 21$ px 28.729

c 22 A

$\begin{array}{llr}\star 22 & p & 0.5 \\ \star 24 & p & -0.5 \\ \star 25 & p & 0.5 \\ & \text { p } & -0.5\end{array}$

$\begin{array}{lll}c & & \\ 36 & \text { D2 } & -42.38\end{array}$

$\begin{array}{llr}37 & \text { pz } & 100.00 \\ 38 & \text { pz } & -100.00\end{array}$

World

mode $n$

kcode $\quad 3000 \quad 1.0 \quad 10 \quad 100$

$\begin{array}{lllll}\text { ksrC } & -8.150 & 0.00 & -33,100\end{array}$

c Cemented Waste $(1.024 \mathrm{~g} / \mathrm{cc}) 65 \%$ Water

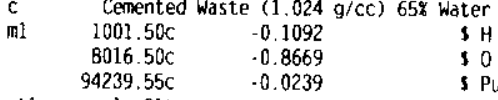

mt.1 poly.01t Hexagonal Surfaces $(A x+B y+C z=0)$

Botton of Orum

Top above Drum

3 Radius of Waste

Bottom of Wast

Top of waste

s Bottom of Can

S Top of Can

5 Radius of

s Bottom of

s Top of Interior

5 Radius of Liner s Bottom of Liner Top of Liner

5 Radius of Drum

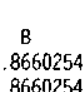

0.866025

0.8660254

0.8660254

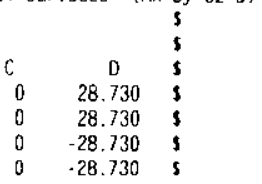

s Concrete Floor

STop of the world

Bottom of the

\begin{tabular}{|c|c|c|c|}
\hline & $18000.35 \mathrm{c}$ & -0.00934 & $\$$ ArC \\
\hline$c$ & \multicolumn{3}{|c|}{ Polyethylene $(0.92 \mathrm{~g} / \mathrm{CC})$} \\
\hline$\pi 4$ & $6000.50 \mathrm{C}$ & 0.03952 & s $\mathrm{C}$ \\
\hline mt 4 & \multicolumn{2}{|l|}{$\begin{array}{l}1001.50 \mathrm{c} \\
\text { poly.01t }\end{array}$} & $5 \mathrm{H}$ \\
\hline \multicolumn{4}{|c|}{ poly.01t } \\
\hline c & \multicolumn{2}{|c|}{ Concrete $(2.24 \mathrm{~g} / \mathrm{cc})$} & \\
\hline \multirow[t]{10}{*}{ m5 } & $1001.50 \mathrm{c}$ & -0.009959 & $S \mathrm{H}$ \\
\hline & $6000.50 \mathrm{c}$ & -0.000979 & s 6 \\
\hline & $8016.50 \mathrm{C}$ & -0.529069 & 50 \\
\hline & $11023.50 \mathrm{c}$ & -0.016003 & S $\mathrm{Na}$ \\
\hline & $12000.50 \mathrm{c}$ & -0.002000 & $5 \mathrm{Mg}$ \\
\hline & $13027.50 \mathrm{c}$ & -0.034006 & S Al \\
\hline & $14000.50 \mathrm{C}$ & -0.337036 & s $\mathrm{Si}$ \\
\hline & $19000.50 \mathrm{c}$ & $-0.01298]$ & Sk \\
\hline & $20000.50 \mathrm{C}$ & .0 .043965 & S $\mathrm{Ca}$ \\
\hline & $26000.55 c$ & -0.014002 & $5 \mathrm{Fe}$ \\
\hline \multirow{2}{*}{\multicolumn{4}{|c|}{ poly.01t }} \\
\hline & & & \\
\hline \multirow{3}{*}{$\begin{array}{l}c \\
m 6\end{array}$} & Water $(1.0 \mathrm{~g}$ & $1 /(C)$ & \\
\hline & $1001.50 \mathrm{c}$ & .0 .1119 & S H \\
\hline & $8016.50 \mathrm{c}$ & -0.8881 & $\$ 0$ \\
\hline \multirow{2}{*}{\multicolumn{4}{|c|}{ S Light Water }} \\
\hline & & & \\
\hline \multirow{4}{*}{$\begin{array}{l}c \\
\text { an } 7\end{array}$} & Cellulose (1. & $.45 \mathrm{~g} / \mathrm{Cc})$ & \\
\hline & $1001.50 \mathrm{c}$ & 0.054311 & S Cellulose Reflector \\
\hline & $8016.50 \mathrm{c}$ & 0.026949 & \\
\hline & $6000.50 \mathrm{c}$ & 0.03233 & \\
\hline \multirow{2}{*}{\multicolumn{4}{|c|}{ poly.01t }} \\
\hline & & & \\
\hline \multirow{2}{*}{$\begin{array}{l}c \\
m 8\end{array}$} & Polyethylent & $\mathrm{e}(0.92 \mathrm{~g} / \mathrm{cc})$ & \\
\hline & $\begin{array}{l}6000.50 \mathrm{c} \\
1001.50 \mathrm{c}\end{array}$ & 0.03952 & s Polyethylene Reflecto \\
\hline $\mathrm{mt8}$ & $\begin{array}{l}1001.50 \mathrm{C} \\
\text { poly.01t }\end{array}$ & 0.07903 & \\
\hline C & & & \\
\hline$c$ & Water $(1.02 \mathrm{~g}$ & $g /(c)$ & \\
\hline \multirow[t]{3}{*}{$m 9$} & $1001.50 \mathrm{c}$ & -0.1097 & $\$ \mathrm{H}$ \\
\hline & $8016.50 \mathrm{c}$ & -0.8703 & \\
\hline & $94239.55 \mathrm{C}$ & -0.0200 & s $\mathrm{Pu}$ \\
\hline $\mathrm{mt} 9$ & Iwtr.01t & & S Light. Water \\
\hline \multicolumn{4}{|l|}{$\begin{array}{l}c \\
\text { totnu }\end{array}$} \\
\hline $\begin{array}{l}\text { totnu } \\
\text { ctme }\end{array}$ & 480 & & \\
\hline print & & & \\
\hline
\end{tabular}

Three Billets in a Non-pipe 55 Gallon Drum

c tiree $66.7 \mathrm{~g}$ Pu billets at the bottom of a $200 \mathrm{~g} / \mathrm{drum}$ c Filled with poly Silicon replaces Calcium

C $4.5 \mathrm{~kg}$ of $\mathrm{Pu}$ as dry oxide $(H / P u=0)$ at theoretical density in cans $(\mathrm{H} / \mathrm{O}-\mathrm{l})$

c. Five cans are in a five position wagon with poly around it

Cell Definitions

Cerrented Can Contents . Universe 1

$\begin{array}{llll}1-1.024-1 \quad 2-3 & u=1 & i m p: n=1 & \text { s Cemented Waste } \\ 3-0.001291:-2: 3^{-3} & u=1 & i m p: n=1 & \text { s steel Can }\end{array}$

Wagon Can Contents - Universe 2

$8 \cdot 11.46 \quad-208204 \cdot 205 \quad u=2 \quad$ imp: $n=1 \quad$ s Plutonium 0xide $3-0.00129$ 201:-204:206 $u=2$ imp: $n=1$ s Can

$\begin{array}{lllll}3 & -0.00129 & -201 & 204 & -206\end{array}$ *3 $u=2 \quad$ imp: $n=1 \quad$ S Alr above Oxide

Cemented Caris in Drum

$\begin{array}{lllllll}3 & -0.00129 & -4 & 5 & -6 & \text { fill-1 } & \text { imp }: n=1\end{array}$ $\operatorname{trcl}=\left(\begin{array}{ccc}-6 \\ 8.150 \quad 0.00 & -20.100)\end{array}\right.$

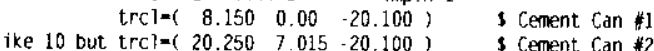

like 10 but $\operatorname{trcl}-(20.250-7.015-20.100)$

Cans in wagon

$3-0.00129-202203 \cdot 207$ fill-2 imp:n-1 trcl $=(37.259-24.158 \cdot 19.200)$

like 13 but $\operatorname{trcl}=(52.614-12.079-19.200)$

like 13 but $\operatorname{trcl}=\left(\begin{array}{lll}37.259 & 0.0 & -19.200\end{array}\right)$

like 13 but $\operatorname{trc}=\left(\begin{array}{llll}52.614 & 12.079 & -19.200\end{array}\right)$

like 13 but $\operatorname{trcl}=(37.259 \quad 24.158 \cdot 19.200)$

Wagon Can \#

5 Wagon Can

3 Wagon Can \#4

Drum

$4-0.92-78-9$ - $10 \# 11 \# 12$ ino:n-1 s Interior 
HNF-6179 Rev. 0

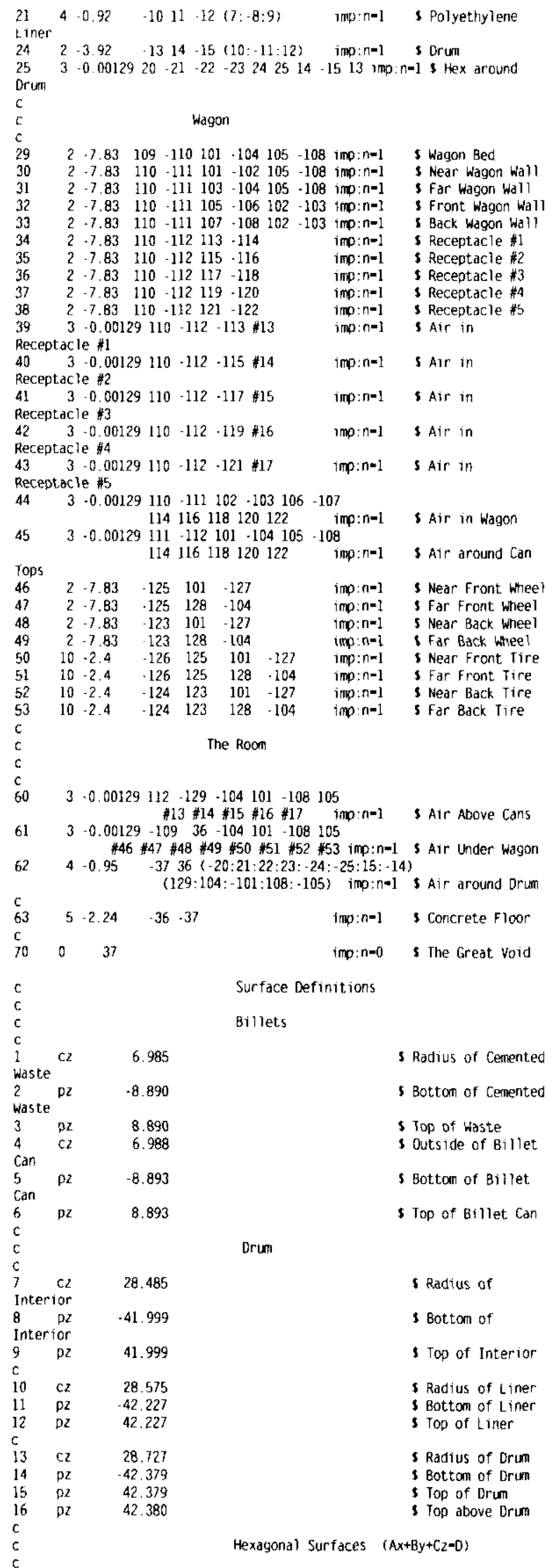

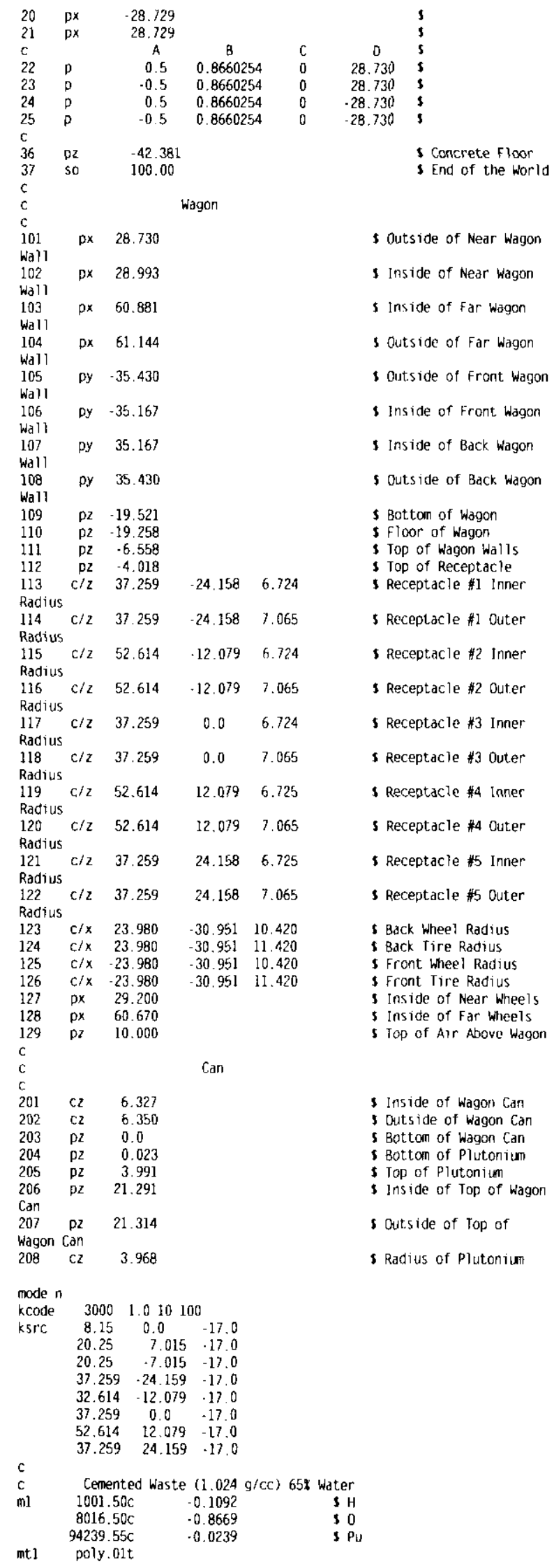


HNF-6179 Rev. 0

\begin{tabular}{|c|c|c|c|}
\hline \multirow{9}{*}{$\mathrm{m} 2$} & SS-304 $\quad 17.8$ & $83 \mathrm{~g} /(\mathrm{C})$ & \\
\hline & $6000.50 c$ & 0.000293 & $s c$ \\
\hline & $14000.50 \mathrm{c}$ & -0.007497 & si si \\
\hline & $15031.50 \mathrm{C}$ & .0 .000447 & S $\mathrm{p}$ \\
\hline & $16032.50 \mathrm{c}$ & -0.000294 & 55 \\
\hline & $24000.50 \mathrm{C}$ & -0.200003 & $\mathrm{sr}$ \\
\hline & $25055.50 \mathrm{c}$ & -0.020000 & s $\mathrm{Mn}$ \\
\hline & $26000.55 \mathrm{c}$ & -0.651464 & $3 \mathrm{Fe}$ \\
\hline & $28000.50 \mathrm{c}$ & -0.120002 & $3 \mathrm{Ni}$ \\
\hline \multicolumn{4}{|c|}{ Air $(0.00129 \mathrm{~g} / \mathrm{CC})$} \\
\hline \multirow[t]{3}{*}{$\mathrm{m} 3$} & $\begin{array}{l}\text { Air } 10.00129 \\
7014.50 \mathrm{C}\end{array}$ & $\begin{array}{l}9 \mathrm{~g} / \mathrm{CC}) \\
-0.78084\end{array}$ & $5 \mathrm{~N}$ \\
\hline & $8016.50 \mathrm{c}$ & -0.20982 & $\begin{array}{l}5 \mathrm{~N} \\
5 \mathrm{O}\end{array}$ \\
\hline & $18000.35 \mathrm{C}$ & -0.00934 & S Arc \\
\hline \multicolumn{4}{|c|}{ 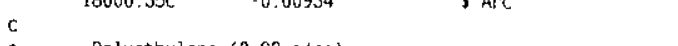 } \\
\hline \multirow{2}{*}{$\mathrm{m} 4$} & $6000.50 \mathrm{c}$ & $\begin{array}{l}0.92 \mathrm{~g} / \mathrm{Cl} \\
0.03952\end{array}$ & $5 \mathrm{C}$ \\
\hline & $1001.50 \mathrm{C}$ & 0.07903 & S \\
\hline \multirow{2}{*}{\multicolumn{4}{|c|}{ poly.01t. }} \\
\hline & Concrete $(2$. & & \\
\hline \multirow{10}{*}{ m5 } & $1001.50 \mathrm{C}$ & $\begin{array}{l}.24 \mathrm{~g}(\mathrm{CC}) \\
-0.009959\end{array}$ & 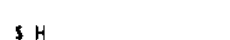 \\
\hline & $6000.50 \mathrm{c}$ & -0.000979 & S $\mathrm{C}$ \\
\hline & $8016.50 \mathrm{c}$ & -0.529069 & $\begin{array}{l}50 \\
50\end{array}$ \\
\hline & $11023.50 \mathrm{c}$ & -0.016003 & $\because \mathrm{Na}$ \\
\hline & $12000.50 \mathrm{c}$ & -0.002000 & $5 \mathrm{mg}$ \\
\hline & $13027.50 \mathrm{c}$ & -0.034006 & i Al \\
\hline & $14000.50 \mathrm{C}$ & -0.337036 & s si \\
\hline & $19000.50 \mathrm{C}$ & .0 .012981 & $3 k$ \\
\hline & $20000.50 \mathrm{C}$ & .0 .043965 & $\$ \mathrm{Ca}$ \\
\hline & $26000.55 \mathrm{c}$ & .0 .014002 & $\$ \mathrm{Fe}$ \\
\hline \multirow{2}{*}{\multicolumn{4}{|c|}{ poly.01t }} \\
\hline & Water $(1.0 \mathrm{~g}$ & $g /(c)$ & \\
\hline \multirow[t]{2}{*}{$\begin{array}{l}\mathrm{C} \\
\mathrm{m} 6\end{array}$} & $1001.50 \mathrm{c}$ & -0.1119 & $5 \mathrm{H}$ \\
\hline & $8016.50 \mathrm{c}$ & -0.8881 & 50 \\
\hline \multirow{2}{*}{\multicolumn{4}{|c|}{ s Light Water }} \\
\hline & & & \\
\hline \multirow[t]{3}{*}{$\pi 7$} & $\begin{array}{l}\text { Celluiose } \\
1001.50 \mathrm{c}\end{array}$ & $\begin{array}{c}1.45 \mathrm{~g} / \mathrm{CC}) \\
0.054311\end{array}$ & \\
\hline & $8016.50 \mathrm{c}$ & 0.026949 & S Cellulose Reflector \\
\hline & $6000.50 \mathrm{c}$ & 0.03233 & \\
\hline \multicolumn{4}{|c|}{ poly.01t } \\
\hline \multirow{2}{*}{$\begin{array}{l}c \\
m B\end{array}$} & Plutonium D & Doixide $(11.46 \mathrm{~g} / \mathrm{Cc})$ & \\
\hline & $\begin{array}{r}94239.55 \mathrm{C} \\
8016.50 \mathrm{c}\end{array}$ & $\begin{array}{l}-0.881 \\
0.219\end{array}$ & $\begin{array}{l}\text { 5 Plutonium } \\
\text { s Oxygen }\end{array}$ \\
\hline \multicolumn{3}{|c|}{$00.506=0.213$} & \\
\hline \multirow[t]{3}{*}{ m9 } & $1001.50 \mathrm{C}$ & -0.1097 & \\
\hline & $8016.50 \mathrm{C}$ & -0.8703 & 30 \\
\hline & $94239.55 \mathrm{C}$ & .0 .0200 & s $\mathrm{Pu}_{\mathrm{u}}$ \\
\hline $\mathrm{mt} 9$ & iwtr.01t & & s Light Water \\
\hline \multicolumn{4}{|r|}{ - congra mater } \\
\hline \multirow[t]{2}{*}{ m10 } & \multicolumn{3}{|c|}{ Rubber $(2.4 \mathrm{~g} / \mathrm{cc})$} \\
\hline & $\begin{array}{l}1001.50 c \\
6012.50 c\end{array}$ & $\begin{array}{l}0.66667 \\
0.33333\end{array}$ & ic \\
\hline $\begin{array}{l}m \mathrm{mt} 10 \\
\mathrm{c}\end{array}$ & poly.01t & & \\
\hline $\begin{array}{l}\text { totnu } \\
\text { ctme }\end{array}$ & 480 & & \\
\hline print & & & \\
\hline
\end{tabular}

nonpipe2_wagon. inp

Three Billets in a Non-pipe 55 Gallon Drum

c three $66.7 \mathrm{~g}$ Pu billets at the bottom of a $400 \mathrm{~g} / \mathrm{drum}$

c Filled with poly Silicon replaces Calcium

c $400 \mathrm{~g}$ of $\mathrm{Pu}$ as wet oxide in cans $(\mathrm{H} / \mathrm{D}=1)$

c Five cans are in a five position wagon with poly around it

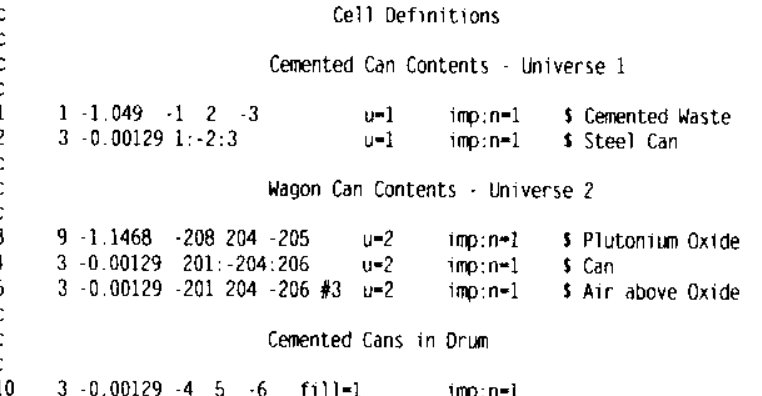

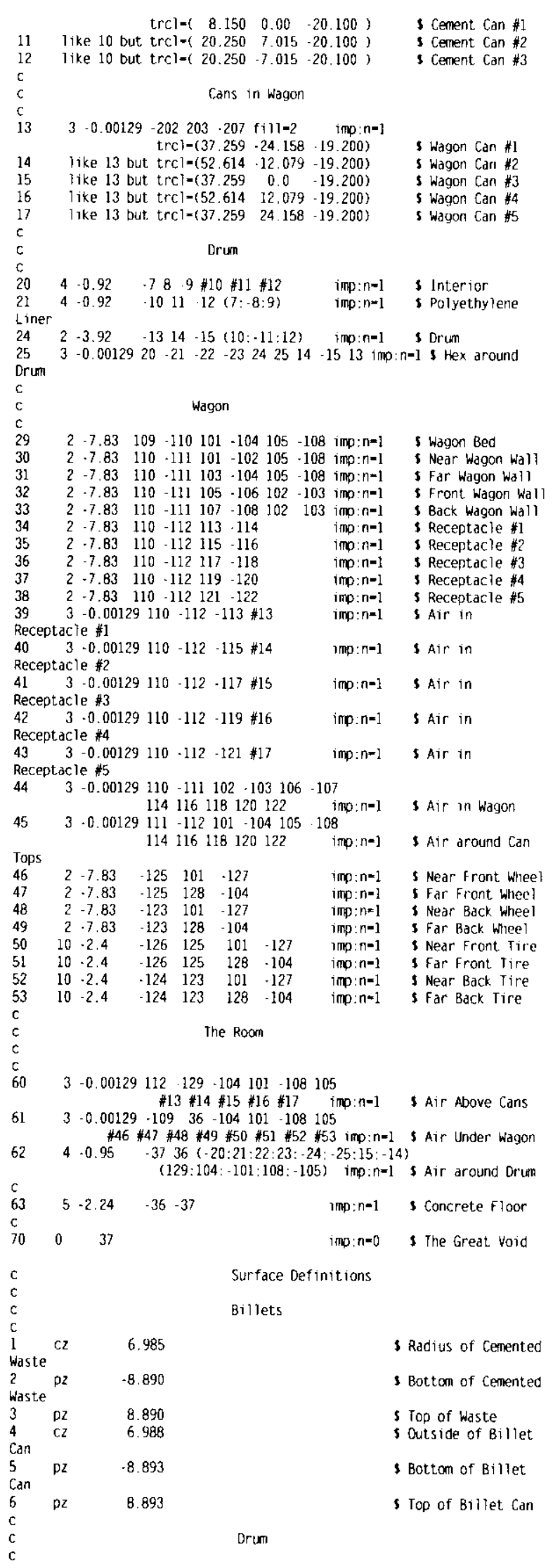




\section{HNF-6179 Rev. 0}

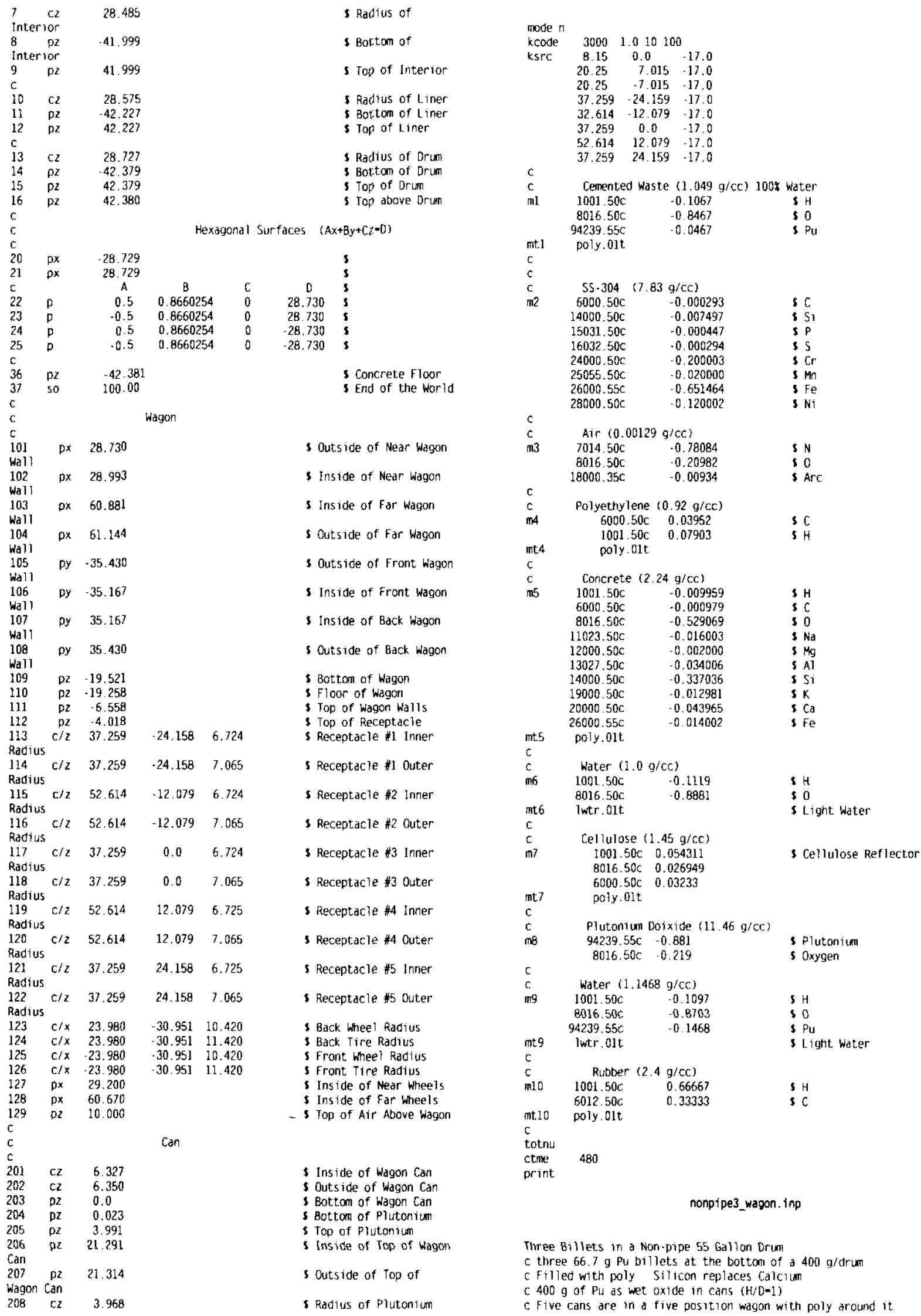


HNF-6179 Rev. 0

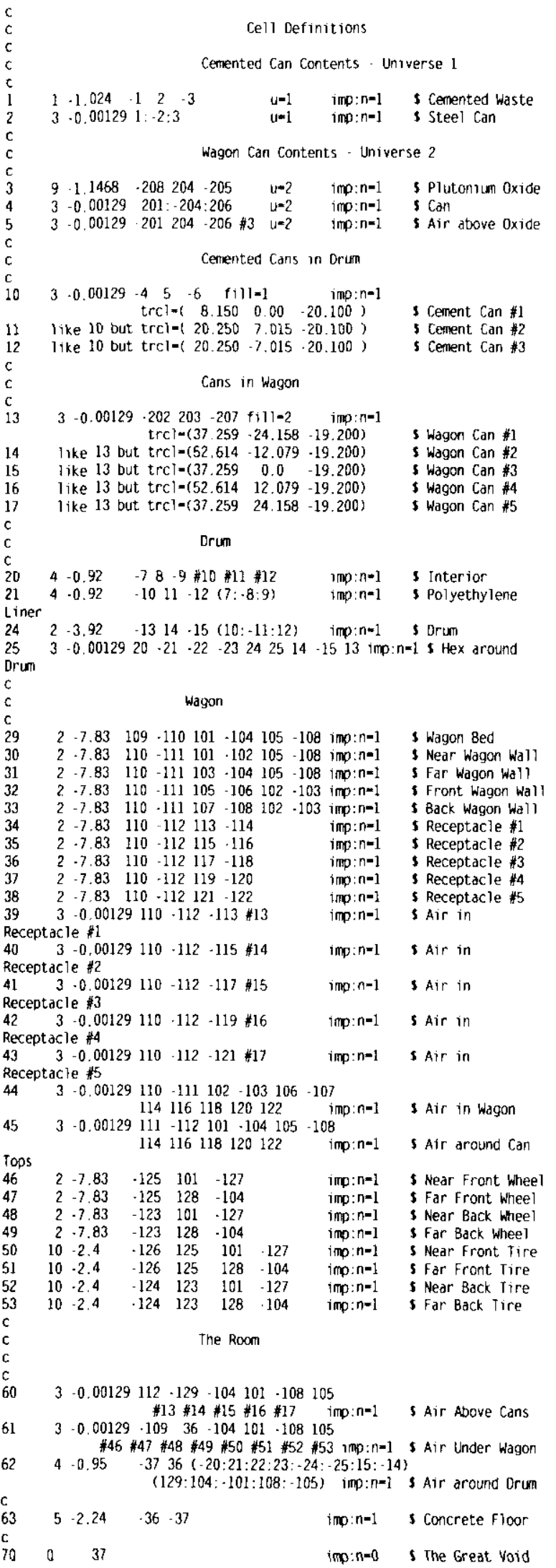

$c$

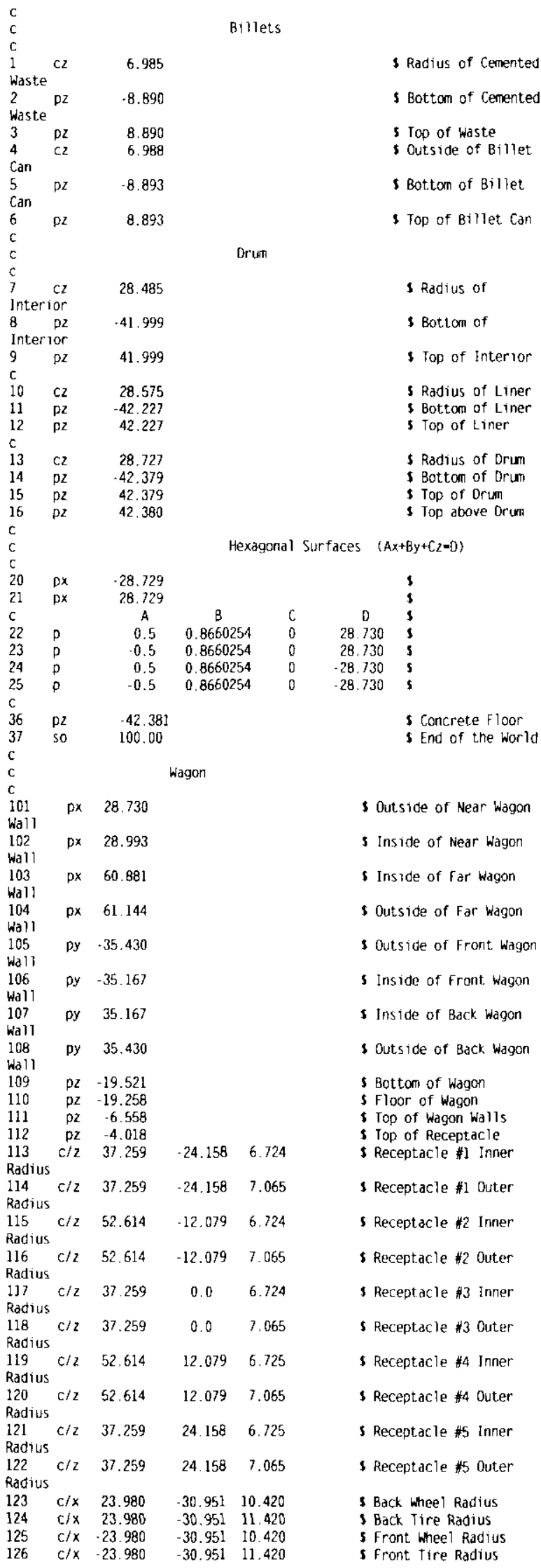


HNF-6179 Rev. 0

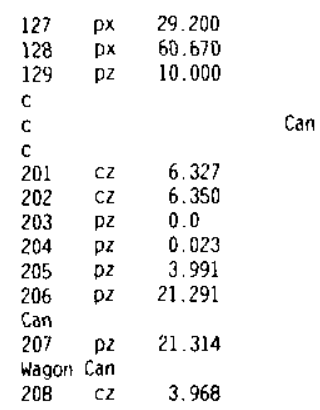

$\begin{array}{lrcr}\text { mode n } & & & \\ \text { kcode } & 3000 & 1.010 & 100 \\ \text { ksrc } & 8.15 & 0.0 & -17.0 \\ & 20.25 & 7.015 & -17.0 \\ & 20.25 & -7.015 & -17.0 \\ & 37.259 & -24.159 & -17.0 \\ & 32.614 & -12.079 & -17.0 \\ & 37.259 & 0.0 & -17.0 \\ & 52.614 & 12.079 & -17.0 \\ & 37.259 & 24.159 & -17.0\end{array}$

c

c Cemented Waste $(1.024 \mathrm{~g} / \mathrm{cc}) 65 \%$ water

ml $\quad \begin{array}{lll}1001.50 \mathrm{C} & -0.1092 & \text { S H }\end{array}$

$\begin{array}{rrr}84239.55 \mathrm{c} & -0.0239 & 5 \mathrm{Pu}\end{array}$

poly.01t

$\begin{array}{lll}c & & \\ c & 55-304 \quad(7.83 \mathrm{~g} / \mathrm{cc})\end{array}$

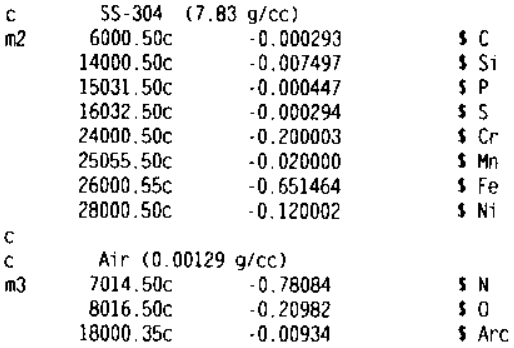

$\begin{array}{lrrr}\mathrm{c} & & & \\ \mathrm{c} & \text { Polyethylene } & (0.92 \mathrm{~g} / \mathrm{cc}) & \\ \text { ind } & 6000.50 \mathrm{C} & 0.03952 & 5 \mathrm{C} \\ & 1001.50 \mathrm{C} & 0.07903 & 5 \mathrm{H}\end{array}$

mt4 poly.01t

$\begin{array}{lccc}\text { c } & & \\ \text { c } & \text { Concrete } & (2.24 \mathrm{~g} / \mathrm{cc}) & \\ \text { m5 } & 1001.50 \mathrm{C} & -0.009959 & \$ \mathrm{H}\end{array}$

$\begin{array}{lll}1001.50 \mathrm{C} & -0.009959 & \text { SH } \\ 6000.50 \mathrm{C} & -0.000979 & \text { SC }\end{array}$

$8016.50 \mathrm{C}-0.529069 \quad$ \$ 0

$11023.50 \mathrm{C}-0.016003 \quad$ S Na

$12000.50 \mathrm{C}-0.002000 \quad 3 \mathrm{Mg}$

$13027.50 \mathrm{C} \quad-0.034006 \quad$ SAl

$14000.50 \mathrm{C}-0.337036 \quad$ Si

$\begin{array}{lll}19000.50 \mathrm{C} & -0.012981 & \text { S K } \\ 20000.50 \mathrm{C} & -0.043965 & \text { s C }\end{array}$

$26000.55 \mathrm{C} \quad-0.014002 \quad$ S Fe

$\mathrm{mt5}$ poly.01t

$\begin{array}{lll}\mathrm{c} & \text { Water }(1.0 \mathrm{~g} / \mathrm{Cc}) \\ \mathrm{c} & 1001.50 \mathrm{C} & -0.1119\end{array}$

$\begin{array}{lll}1001.50 \mathrm{C} & -0.1119 & \text { S } \mathrm{H} \\ 8016.50 \mathrm{C} & -0.8881 & \text { 3 } 0\end{array}$

mt6 iwtr.01t s Light water

c Cellulose $(1.45 \mathrm{~g} / \mathrm{cc})$

$1001.50 \mathrm{C} \quad 0.054311$

$8016.50 \mathrm{C} \quad 0.026949$

s Celluiose Reflector

int 7 poly.01t

c $\quad$ Plutoniun Doixide $(11.46 \mathrm{~g} / \mathrm{CC})$

m8 94239.55c -0.881 s Plutonitum

c Water $(1.1468 \mathrm{~g} / \mathrm{cc})$

c Water ( $1.1468 \mathrm{~g} / \mathrm{cc})$

$\begin{array}{ll}1001.50 \mathrm{C} & -0.1097 \\ 8016.50 \mathrm{C} & .0 .8703\end{array}$

$94239.55 \mathrm{C}$

-0.8703
-0.1468

loter. ol

soxygen

Inside of Wagon Can

Cutside of Wagon Can

Bottom of Wagon Can

Bottom of Plutoniu

$\checkmark$ inside of Top of wagon

5 Outside of Top of

s Radius of Plutonium

Ruboer $(2.4 \mathrm{~g} / \mathrm{cc})$

$\begin{array}{llll}\text { mlo } & 1001.50 \mathrm{C} & 0.66667 & \text { S H } \\ & 6012.50 \mathrm{c} & 0.33333 & \text { S } \\ \text { mt10 } & \text { poly.01t } & & \\ \mathrm{c} & & & \\ \text { totnu } & & & \\ \text { ctme } & 480 & & \\ \text { print. } & & & \end{array}$

nonpipe4_wagon.inp

Three Billets in a Non-pipe 55 Gallon Drum

c three $66.7 \mathrm{~g}$ Pu billets at the bottom of a $400 \mathrm{~g} / \mathrm{drum}$

$c$ filled with poly silicon replaces Calcium

c $400 \mathrm{~g}$ of $\mathrm{Pu}$ as wet oxide in cans $(\mathrm{H} / \mathrm{D}-1)$

c Five cans are in a five position wagon with poly around it

Cell definitions

Cemented Cari Contents - Universe

$\begin{array}{llll}-1.049 & -1 & 2 & -3 \quad u=1 \\ 3\end{array}$

$3-0.001291: \cdot 2: 3^{-3} \quad u=1 \quad$ imp: $n=1 \quad$ s steel Can

Wagon Can Contents - Universe 2

B $-11.46-208204-205 \quad u=2 \quad$ imp:n-1 B Plutonium Oxide

$3-0.00129$ 201:-204:206 u=2 imo:n-1 s Can

$3-0.00129-201204 \cdot 206 \$ 3 \quad u=z \quad$ inp:n=1 S Air above $0 x i d c$

Cemented Caris in Drum

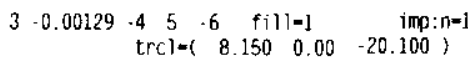

like 10 but tral-( $20.250-7.015-20.100)$

Cans in Wagon

$3-0.00129-202203-207$ fill $=2$ imp:n-1 trcl $=(37.259 \cdot 24.158-19.200)$

like 13 but $\operatorname{trcl}=(52.614 \cdot 12.079-19.200)$

like 13 but $\operatorname{trcl}=(37.259 \quad 0.0 \quad-19.200)$

like 13 but $\operatorname{trc})=(52.614 \quad 12.079-19.200)$

$s$ wagon Can \#1 5 wagon Can \#2

Wagon Can $\$ 4$

5 Wagon Can *5

Oruin

$4-0.92 \quad-78-9 \# 10 \# 11 \# 12$ imp:n-1 s Interior

$4 \cdot 0.92-1011-12(7:-8: 9)$ imp:n-1, Polyethylene

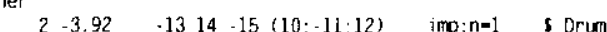

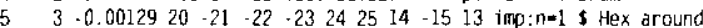

Drum

c Wagon

$2 \cdot 7.83 \quad 109-110 \quad 101-104 \quad 105-108$ imp: $n=1$

$2.7 .83 \quad 110-111 \quad 101-102 \quad 105-108$ imp: $n=1$ s Near Wagon Wall

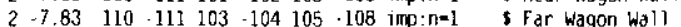

$\begin{array}{llllllllllll}2 & -7 & 83 & 110 & -111 & 105 & -106 & 102 & -103 & \text { imp:n-1 S Front Wagon Wa } 11\end{array}$

$\begin{array}{llllllllllll}2 & -7.83 & 110 & -111 & 107 & -108 & 102 & -103 & \text { imp: }: n-1 & \text { s Back Wagon wall }\end{array}$

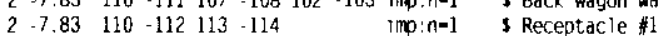

$\begin{array}{lllllll}2 & -7.83 & 110 & -112 & 115 & -116\end{array}$

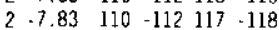

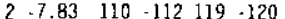

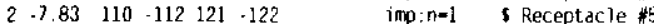

$3-0.00129110-112-113 \# 13 \quad$ imo:nal s A 13 in

Receptacle

$40 \quad 3-0.00129110-112-115 * 14 \quad$ imp:n=1 S Air in

Receptacle \#2

$41 \quad 3-0.00129110-112-117$ \#15 imp:n=1 Air in

Receptacle \#3

$42 \quad 3 \cdot 0.00129110-112-119 \# 16 \quad$ imp:n=1 S Air in

Receptacle

$43 \quad 3 \cdot 0.00129110-112 \cdot 121 \# 17$ imp:n=1 sir in

Receptacle

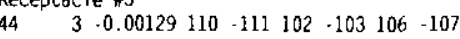

$45 \quad 3-0.00129111 \quad-112 \quad 101-104 \quad 105-108$ s Air around Can

Tops

$\begin{array}{lllll}2 & -7.83 & -125 & 101 & -127\end{array}$

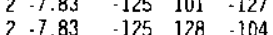

$\begin{array}{lllll}2 & -7.83 & -125 & 128 & -104 \\ 2 & -7.83 & -123 & 101 & -127\end{array}$

$\begin{array}{lllll}2 & -7.83 & .123 & 101 & -127 \\ 2 & -7.83 & .123 & 128 & -104\end{array}$

$\begin{array}{lllll}2 & -7.83 & .123 & 128 & -104\end{array}$

$\begin{array}{llllll}10 & -2.4 & -126 & 125 & 107 & -127\end{array}$

$\begin{array}{llllll}10 & -2.4 & .126 & 125 & 128 & .104\end{array}$

$\begin{array}{llllll}10 & -2.4 & -124 & 123 & 101 & .127\end{array}$

$\begin{array}{llll}124 & 123 & 128 & -104\end{array}$

\section{ino $: n=1$}

imp: $n=1$, Far Front wheel

imo:n=1 S Near Back wheel

imp: $n=1$ S Far Back wheel

Imp: $n=1$ S Near Front. Tire

$m p: n=1$ S Far Front Tire

man: $n=1$ \$ Near Back Trre

imo:n-1 S Far Back Tire 
HNF-6179 Rev. 0

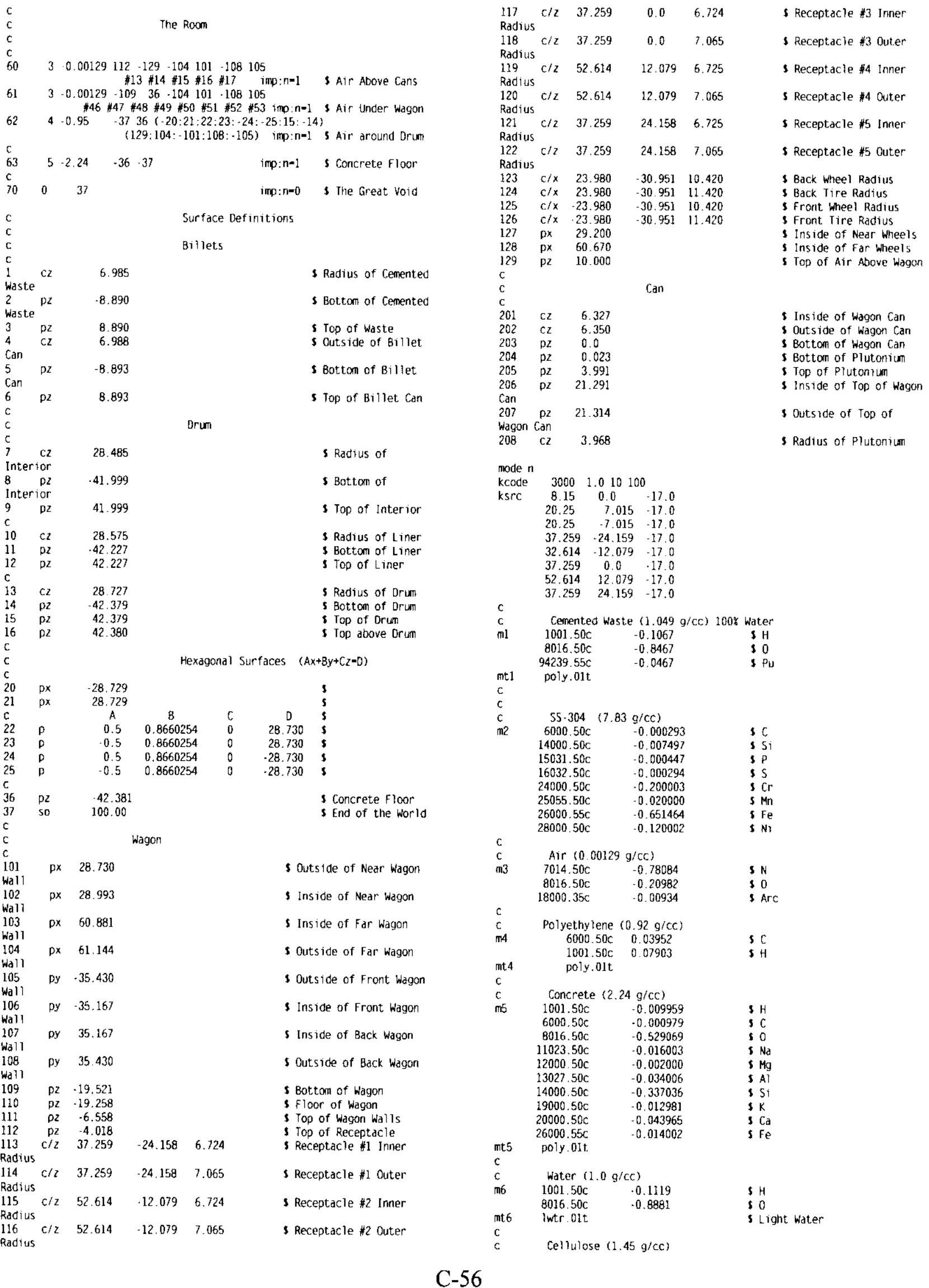


HNF-6179 Rev. 0

\begin{tabular}{|c|c|c|c|}
\hline$m 7$ & $\begin{array}{l}1001.50 c \\
8016.50 c \\
6000.50 c\end{array}$ & $\begin{array}{l}0.054311 \\
0.026949 \\
0.03233\end{array}$ & 5 Cellulose Reflector \\
\hline mit.7 & poly.01t & & \\
\hline $\mathrm{c}$ & \multirow{2}{*}{\multicolumn{2}{|c|}{ Plutonium Doixide $(11.46 \mathrm{~g} / \mathrm{CC})$}} & \\
\hline$c$ & & & \\
\hline $\mathrm{mg}$ & $\begin{array}{r}94239.55 c \\
8016.50 \mathrm{c}\end{array}$ & $\begin{array}{l}-0.881 \\
-0.219\end{array}$ & $\begin{array}{l}\text { \$ Plutonium } \\
\text { \$ Oxygen }\end{array}$ \\
\hline$c$ & \multirow{2}{*}{\multicolumn{2}{|c|}{ Water $(1.1468 \mathrm{~g} / \mathrm{cc})$}} & \\
\hline c & & & \\
\hline $\mathrm{mq}$ & $\begin{array}{r}1001.50 \mathrm{C} \\
8016.50 \mathrm{C} \\
94239.55 \mathrm{C}\end{array}$ & $\begin{array}{r}-0.1097 \\
-0.8703 \\
-0.1468\end{array}$ & $\begin{array}{l}\$ \mathrm{H} \\
\$ 0 \\
\$ \mathrm{Pu}\end{array}$ \\
\hline $\mathrm{mt} 9$ & lwtr. olt & & s Light water \\
\hline$c$ & & & \\
\hline c & \multicolumn{2}{|c|}{ Rubber $(2.4 \mathrm{~g} / \mathrm{Cc})$} & \\
\hline $\operatorname{mil} 0$ & $\begin{array}{l}1001.50 c \\
6012.50 c\end{array}$ & $\begin{array}{l}0.66667 \\
0.33333\end{array}$ & S $\mathrm{H}$ \\
\hline$m+10$ & poly.01t & & \\
\hline $\begin{array}{l}c \\
\text { totnu }\end{array}$ & & & \\
\hline $\begin{array}{l}\text { ctine } \\
\text { print. }\end{array}$ & 480 & & \\
\hline
\end{tabular}

nonpipe5_wagon.inp

Three Billets in a Non-pipe $55 \mathrm{Gal}$ lon Drum

c three $66.7 \mathrm{~g}$ Pu billets at the Dottom of a $200 \mathrm{~g} / \mathrm{drum}$

CFilled with poly silicon replaces Calcium

c $2.5 \mathrm{~kg}$ of Pu as metal (H/Pu=O) in cans (H/D-l)

c Five cans are in a five position wagon with poly around it

c

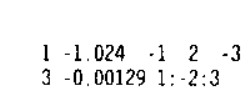

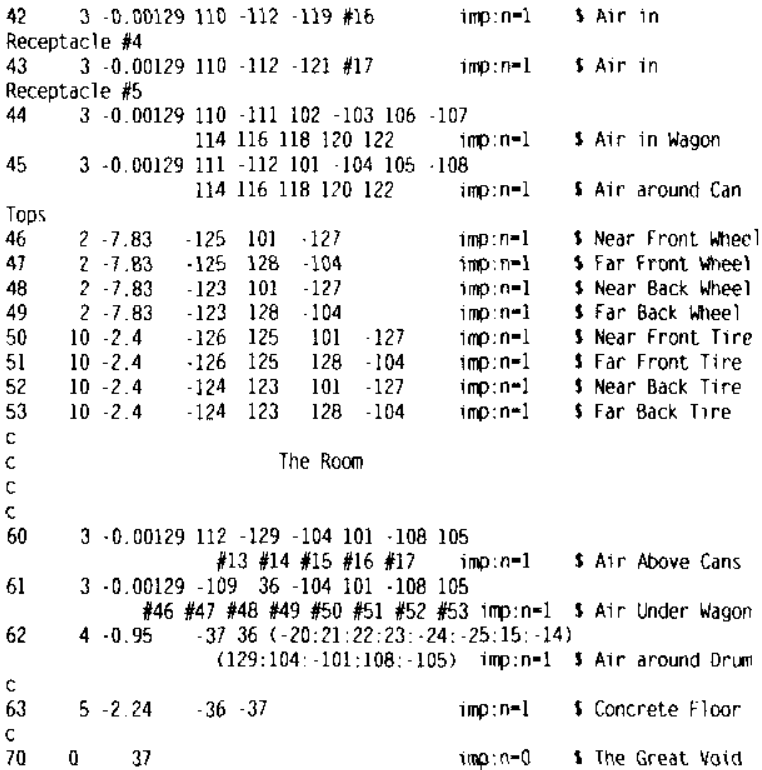

5 Bottom of

s Top of Interior

s Radius of Liner s Bottom of Liner s Top of Liner

S Radius of Drum 5 Botton of Drum S Top of Drum s Top of Drum

Hexagonal Surfaces $(A x+B y+C z=0)$

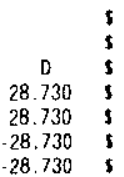

s Concrete Fioor 3 End of the World

S Outside of Near Wagon

s Inside of Near Wagon

5 Inside of Far wagon

$\$$ Outside of far wagon

s Outside of Front wagon 
HNF-6179 Rev. 0

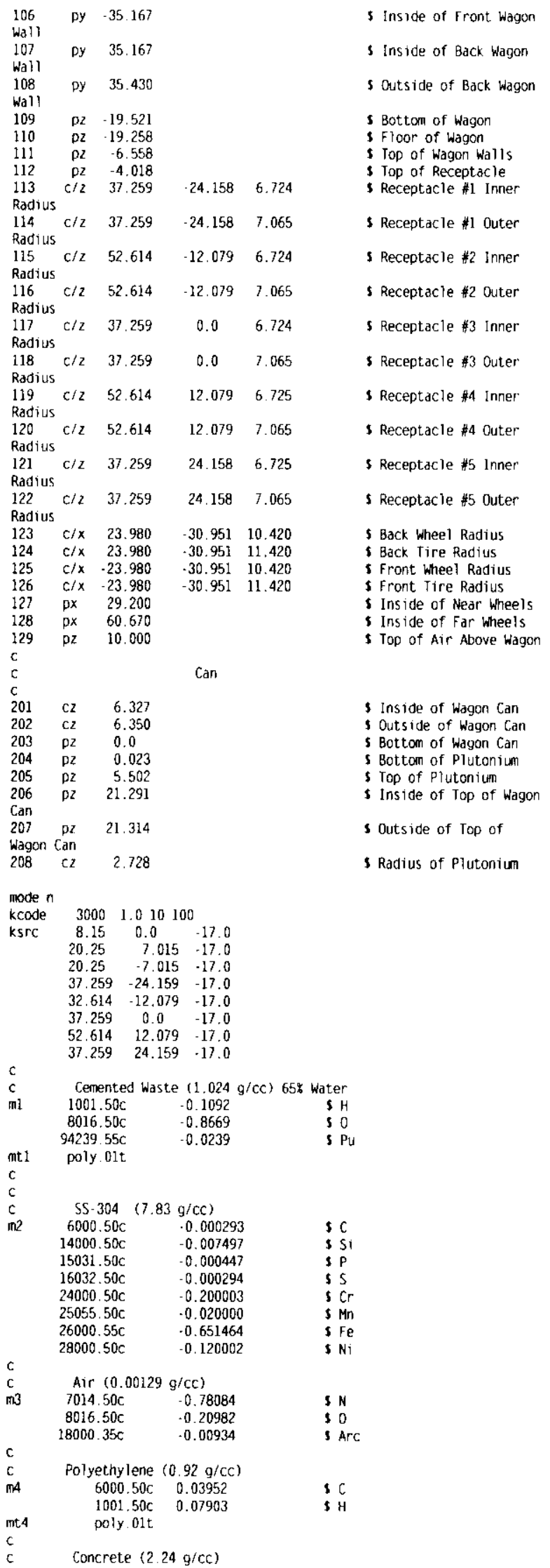

\begin{tabular}{|c|c|c|c|}
\hline \multirow[t]{10}{*}{ m5 } & $1001.50 \mathrm{c}$ & -0.009959 & $\$ \mathrm{k}$ \\
\hline & $6000.50 \mathrm{c}$ & -0.000979 & $5 \mathrm{C}$ \\
\hline & $8016.50 \mathrm{c}$ & -0.529069 & 50 \\
\hline & $11023.50 \mathrm{C}$ & -0.016003 & s $\mathrm{Na}$ \\
\hline & $12000.50 \mathrm{c}$ & -0.002000 & S $\mathrm{Mg}$ \\
\hline & $13027.50 \mathrm{c}$ & -0.034006 & S Al \\
\hline & $14000.50 \mathrm{c}$ & -0.337036 & s Si \\
\hline & $19000.50 \mathrm{C}$ & -0.012981 & $5 \mathrm{k}$ \\
\hline & $20000.50 \mathrm{c}$ & -0.043965 & $5 \mathrm{Ca}$ \\
\hline & $26000.55 c$ & .0 .014002 & $s \mathrm{Fe}$ \\
\hline \multirow{2}{*}{\multicolumn{4}{|c|}{ poly.01t }} \\
\hline & & & \\
\hline \multicolumn{4}{|c|}{ Water $(1.0 \mathrm{~g} / \mathrm{cc})$} \\
\hline \multirow[t]{2}{*}{$\pi 6$} & $1001.50 \mathrm{C}$ & -0.1119 & $5 \mathrm{H}$ \\
\hline & $\mathrm{B} 016.50 \mathrm{C}$ & -0.8881 & so 0 \\
\hline $\mathrm{mt} 6$ & Iwtr.01t & & S Light Water \\
\hline \multicolumn{4}{|r|}{ 2. } \\
\hline c & Cellutose & $(1.45 \mathrm{~g} / \mathrm{CC})$ & \\
\hline \multirow[t]{3}{*}{ my } & $1001.50 \mathrm{c}$ & 0.054311 & - Cellulose Reflector \\
\hline & $8016.50 \mathrm{C}$ & 0.026949 & \\
\hline & $6000.50 \mathrm{C}$ & 0.03233 & \\
\hline $\mathrm{mt} 7$ & poly.01t. & & \\
\hline \multicolumn{4}{|l|}{ c } \\
\hline c & Plutonium & Dolxide $(11.46 \mathrm{~g} / \mathrm{Cc})$ & \\
\hline \multirow[t]{2}{*}{$m 8$} & $94239.55 \mathrm{C}$ & -0.881 & s Plutonitum \\
\hline & $8016.50 \mathrm{c}$ & -0.219 & s oxygen \\
\hline \multicolumn{4}{|l|}{ c } \\
\hline$c$ & Water $(1.02$ & $\mathrm{g} /(\mathrm{cc})$ & \\
\hline \multirow[t]{3}{*}{$\mathrm{mg}$} & $1001.50 \mathrm{c}$ & -0.1097 & S H \\
\hline & $8016.50 c$ & -0.8703 & $\$ 0$ \\
\hline & $94239.55 \mathrm{C}$ & -0.0200 & s Pu \\
\hline $\mathrm{mt} 9$ & Iwtr.01t & & S Light water \\
\hline \multicolumn{4}{|r|}{ - } \\
\hline c & Rubber & $2.4 \mathrm{~g} / \mathrm{cc})$ & \\
\hline \multirow[t]{2}{*}{$\mathrm{m} 10$} & $1001.50 \mathrm{c}$ & 0.66667 & $5 \mathrm{H}$ \\
\hline & $6012.50 \mathrm{C}$ & 0.33333 & $\$ c$ \\
\hline $\mathrm{mt} 10$ & poly.01t & & \\
\hline c & Pu Metal & $(19.6 \mathrm{~g} / \mathrm{cc})$ & \\
\hline m11 & $94239.55 \mathrm{C}$ & -1.000 & $5 \mathrm{Pu}-239$ \\
\hline \multicolumn{4}{|l|}{$\begin{array}{l}c \\
\text { totnu }\end{array}$} \\
\hline $\begin{array}{l}\text { ctme } \\
\text { print }\end{array}$ & 480 & & \\
\hline
\end{tabular}

nonpipe6_wagon. inp

Three Billets in a Non-pipe 55 Gallon Drum $c$ three $66.7 \mathrm{~g}$ Pu billets at the bottom of a $200 \mathrm{~g} / \mathrm{drum}$ c filled with poly silicon replaces Calcium c $2.5 \mathrm{~kg}$ of $\mathrm{Pu}$ as metal (H/Pu=0) in cans (H/D-1) $c$ Five cans are in a five position wagon with poly around it One can overbatched

Cell Definitions

Cemented Can Contents - Universe 1

$\begin{array}{llll}1-1.024-12.3 & u=1 & \text { imp: } n=1 & \text { s Cemented Waste } \\ 3-0.001291:-2: 3 & u=1 & i m p: n=1 & \text { s steel Can }\end{array}$

Wagon Can Contents. Universe 2

$11-19.6 \quad-208204-205 \quad u=2 \quad 1 m p: n=1 \quad$ s Plutonilum Metal $\begin{array}{llll}11-19.6 & -208204-205 & u=2 & 1 m p: n=1 \\ 3-0.00129 & 201 \cdot-204 \cdot 206 & u=2 & i m p: n=1\end{array}$

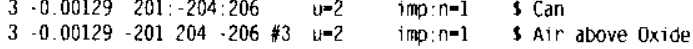
Double Batched Wagon Can Contents - Universe 3

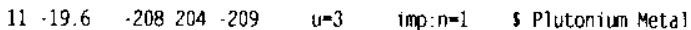
$3-0.00129 \quad 201:-204: 206 \quad \mathrm{u}=3 \quad$ imp: $n=1$ s Can

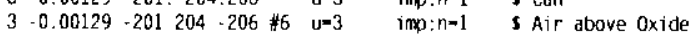

Cemented Cans in Drum

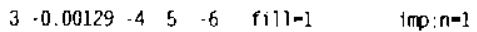

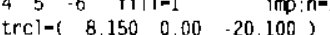

like 10 but trcl-( $20.250 \quad 7.015-20.100)$ $\begin{array}{lll}\text { like } 10 \text { but } \operatorname{trcl}-\left(\begin{array}{rrr}20.250 & 7.015 & -20.100\end{array}\right) & \text { s Cement Can } \# 2 \\ \text { like } 10 \text { but } \operatorname{trcl}=(20.250 & -7.015-20.100) & \text { s Cement Can } \# 3\end{array}$

Cans in Wagon

$3-0.00129-202 \quad 203-207$ firl $=2 \quad$ imp:n-1 trcl-(37.259-24.158-19.200) s Wagon Can \#1 1 ike 13 but trcl $=(52.614-12.079-19.200)$ s wagon Can \#2 $3-0.00129 \cdot 202203-207$ fill-3 imp:n-1 $\begin{array}{ccc}\operatorname{trcl}=(37.259 & 0.0 & -19.200)\end{array}$ s Double Wagon Can

16 like 13 but t,rcl-(52.614 $12.079 \cdot 19.200)$ I Wagon Can *4 
HNF-6179 Rev. 0

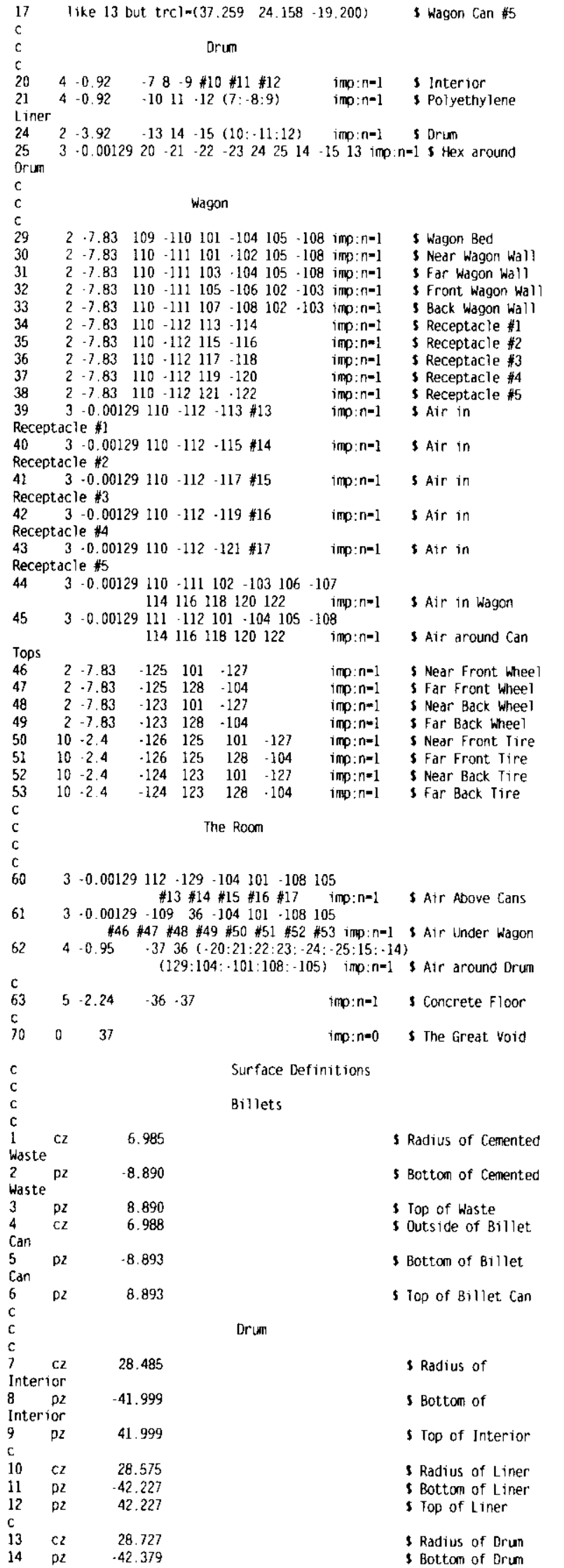

\begin{tabular}{|c|c|c|c|c|c|c|c|}
\hline 15 & $\mathrm{pz}$ & 42.379 & & & & & s \\
\hline 16 & pz & 42.380 & & & & & s \\
\hline c & & & \multirow{2}{*}{\multicolumn{4}{|c|}{ Hexagonal Surfaces }} & \\
\hline c & & & & & & & \\
\hline$c$ & & & & & & & \\
\hline 20 & $\mathrm{px}$ & -28.729 & & & & & \\
\hline 21 & $p x$ & 28.729 & & & & & \\
\hline c & & A & B & C & & D & \\
\hline 22 & $p$ & 0.5 & 0.8660254 & 0 & & .730 & \\
\hline 23 & $p$ & -0.5 & 0.8660254 & 0 & 28.7 & .730 & \\
\hline 24 & p & 0.5 & 0.8660254 & 0 & -28.7 & .730 & \\
\hline 25 & $p$ & -0.5 & 0.8660254 & 0 & -28.7 & .730 & \\
\hline
\end{tabular}

$\begin{array}{llll}36 & \mathrm{pz} & -42.381 & \text { s Concrete floor } \\ 37 & \text { so } & 100.00 & \text { s End of the World } \\ \text { c } & & & \end{array}$

c Wagon

101 px 28.730

$\begin{array}{lll}\text { Wall } & & \\ 102 & \text { px } & 28.993\end{array}$

$\begin{array}{lll}\text { Wall } & & \\ 103 & & \\ \text { px } & 60.881\end{array}$

$\begin{array}{lll}103 & \text { px } & 60.881 \\ \text { Wall } & & \\ 104 & \text { px } & 61.144\end{array}$

$\begin{array}{lll}104 & \text { px } & 61.144 \\ \text { Wa11 } & & \\ 105 & \text { py } & -35.430\end{array}$

$\begin{array}{lll}\text { Wa11 } & & \\ 106 & \text { py } & -35.167\end{array}$

$\begin{array}{lll}106 & \text { py } & -35.167 \\ \text { Wall } & & \\ 107 & \text { py } & 35.167\end{array}$

$\begin{array}{lll}107 & \text { py } & 35.167 \\ \text { Wall } & & \\ 108 & \text { py } & 35.430\end{array}$

$\begin{array}{lll}\text { Wal1 } & & \\ 109 & \text { Dz } & -19.521\end{array}$

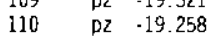

$111 \quad \mathrm{pz}-6.558$

112 pZ -4.018

$113 \mathrm{c} / 2 \quad 37.259$

$114 \quad c / 2 \quad 37.259$

$\begin{array}{ll}\text { Radius } & \\ \text { I15 } & \mathrm{c} / 2 \quad 52.614\end{array}$

$115 \quad \mathrm{c} / 2 \quad 52.614$
Radius

$\begin{array}{lll}\text { Radius } & \\ 116 \quad \mathrm{C} / \mathrm{z} & 52.614\end{array}$

$\begin{array}{lll}\text { Radius } & & \\ 117 & \mathrm{C} / \mathrm{z} & 37.259\end{array}$

$\begin{array}{lll}\text { Radius } & & \\ 118 \quad \text { C/2 } & 37.259\end{array}$

Radius $\begin{array}{ll}\mathrm{C} / 2 & 37.259 \\ & \end{array}$

$\begin{array}{lll}119 & c / 2 & 52.614\end{array}$

$\begin{array}{lll}\text { Radius } & & \\ 120 & \mathrm{C} / \mathrm{Z} & 52,614\end{array}$

Radius

$\begin{array}{lll}121 \quad \mathrm{C} / 2 & 37.259\end{array}$

$122 \mathrm{c} / \mathrm{z} \quad 37.259$

Radius

$123 \mathrm{c} / \mathrm{x} \quad 23.980$

$124 \mathrm{c} / \mathrm{x} \quad 23.980$

$c / x \quad-23.980$

$c / x \quad-23.980$

$127 \quad \mathrm{px} \quad 29.200$

128 px 60.670

129 pz 10.000

$-24.158 \quad 7.065$

$-12.079 \quad 6.724$

$12.079 \quad 7.065$

$0.0 \quad 6.724$

$0.0 \quad 7.065$

$12.079 \quad 6.725$

$12.079 \quad 7.065$

$24.158 \quad 6.725$

$24.158 \quad 7.065$

$30.951 \quad 10.420$

$30.951 \quad 11.420$

$-30.951 \quad 10.420$

$.30 .951 \quad 11.420$

Can

$201 \quad c 2 \quad 6.327$

$202 \quad \mathrm{C2} \quad 6.350$

203 p2 0.0

$204 \quad$ p2 0.023

$\begin{array}{rrr}205 & \text { D2 } & 5.502 \\ 206 & \text { p2 } & 21.291\end{array}$

Can 21.314

Wagon Can

$208 \quad C Z \quad 2.728$

$\begin{array}{llr}209 \quad \text { PZ } & 10.981\end{array}$

$\begin{array}{lrcr}\begin{array}{llcl}\text { mode n } \\ \text { kcode }\end{array} & 3000 & 1.010100 \\ \text { ksrc } & 8.15 & 0.0 & -17.0 \\ & 20.25 & 7.015 & -17.0 \\ & 20.25 & -7.015 & -17.0 \\ & 37.259 & -24.159 & -17.0 \\ & 32.614 & -12.079 & -17.0 \\ & 37.259 & 0.0 & -17.0 \\ & 52.614 & 12.079 & -17.0\end{array}$

Top of Drum

Top above Drum

s Outside of Near Wagon

s Inside of Near Wagon

5 Inside of Far wagon

5 Outside of Far wagon

3 Outside of Front Wagon

5 Inside of Front wagon

5 Inside of Back wagon

s Outside of Back Wagon

Bottom of Wagon

S floor of Wagon

5 Top of Wagon Walls

5 Top of Receptacle

5 Receptacle \#1 Inner

5 Receptacle \#l Outer

- Receptacle \#2 Inner

s Receptacle \#2 Outer

5 Receptacle \#3 Inner

5 Receptacle \#3 Outer

5 Receptacle \#4 Inner

S Receptacle \#4 Outer

s Receptacle \#5 Inner

5 Receptacle \#5 Outer

s Back wheel Radius

s Back Tire Radius

5 Front wheel Radius

5 Front Tire Radius

S Inside of Near wheels

5 Inside of $\mathrm{Far}$ wheels

s Top of Air above Wagon

5 Inside of Wagon Can

5 Outside of Wagon Can

s Bottom of Wagon Can

s Battom of Plutonium

5 Top of Plutonium

s Inside of Top of Wagon

s Outside of Top of

5 Radius of Plutonium 5 Top of Double Batched 
HNF-6179 Rev. 0

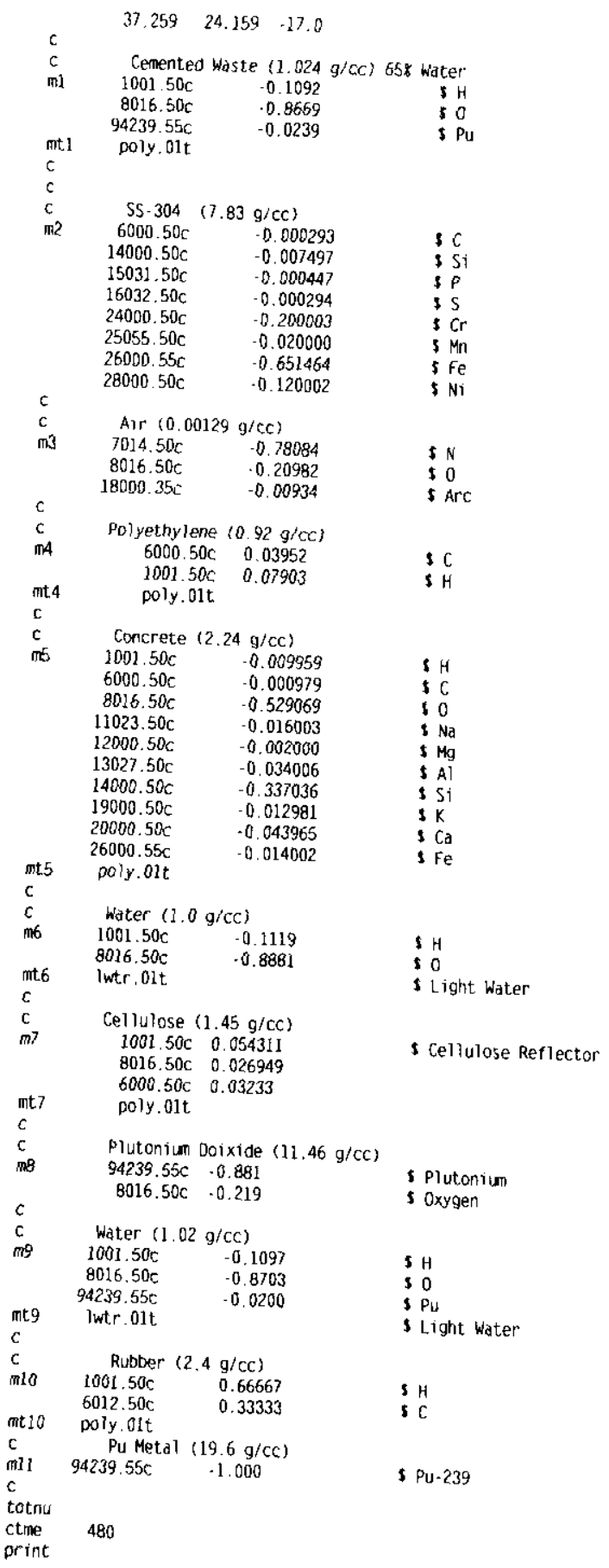

adrun_bot1.ing

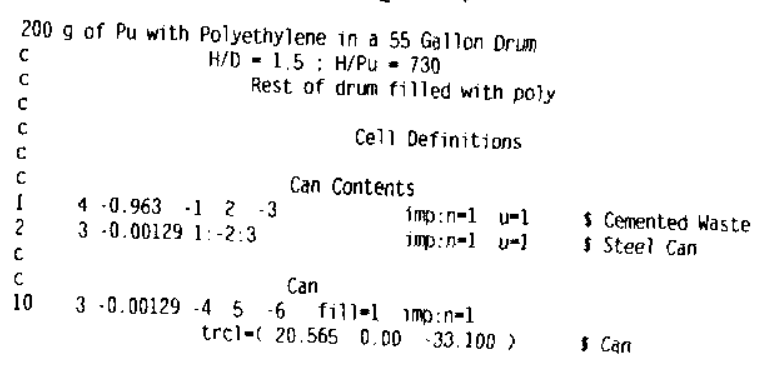

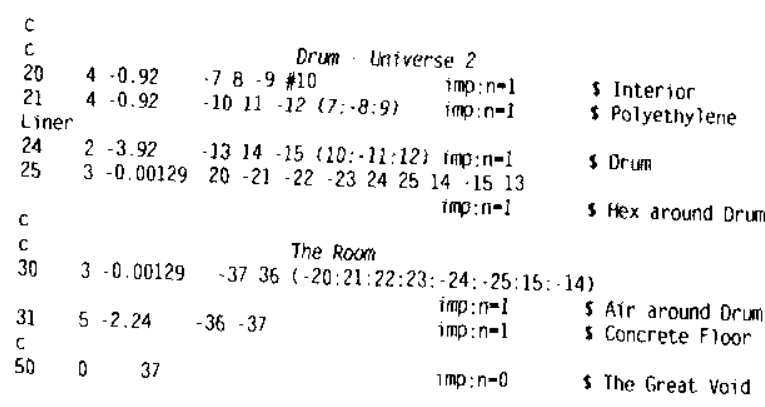

\begin{tabular}{|c|c|c|c|c|c|}
\hline $\begin{array}{l}\mathrm{C} \\
\mathrm{C}\end{array}$ & \multicolumn{5}{|c|}{ Surface Definitrons } \\
\hline c & & & Can & & \\
\hline 1 & $\mathrm{cz}$ & 7.904 & & & \$ Radius of Waste \\
\hline 2 & D $I$ & -8.890 & & & I Botton of Waste \\
\hline 3 & pI & 14.817 & & & S Top of waste \\
\hline 4 & $\mathrm{CZ}$ & 7.907 & & & S Outside of Can \\
\hline 5 & D2 & -8.893 & & & S Bottom of Can \\
\hline $\begin{array}{l}6 \\
c\end{array}$ & $p 2$ & 14.820 & & & s Top of Can \\
\hline c & & & Drum & & \\
\hline Inte & $\begin{array}{l}C 2 \\
\text { ior }\end{array}$ & 28.485 & & & s Radius of \\
\hline 8 & $\begin{array}{l}D 2 \\
\text { ior }\end{array}$ & -41.999 & & & $s$ Bottorn of \\
\hline $\begin{array}{l}9 \\
6\end{array}$ & $D 2$ & 41.999 & & & s Top of Interior \\
\hline 10 & $c z$ & 28.575 & & & S Radius of Liner \\
\hline 11 & Dz & -42.227 & & & s Bottom of Liner \\
\hline $\begin{array}{l}12 \\
c\end{array}$ & $a z$ & 42.227 & & & s Top of Liner \\
\hline 13 & $\zeta z$ & 28.727 & & & \\
\hline 14 & $\mathrm{p} Z$ & -42.379 & & & 3 Radius of Drun \\
\hline 15 & 02 & 42.379 & & & $\$$ Bottom of Drum \\
\hline 16 & D2 & 42.380 & & & $\begin{array}{l}\text { Top of Drum } \\
\text { Top above Drum }\end{array}$ \\
\hline $\begin{array}{l}c \\
c\end{array}$ & & & & & \\
\hline 20 & $p x$ & .28 .729 & Hexag & nal Surfaces & $+B y+C z=0)$ \\
\hline 21 & $\mathrm{Dx}$ & 28.729 & & & 5 \\
\hline$c$ & & A & B & D & $\begin{array}{l}5 \\
5\end{array}$ \\
\hline 22 & D & 0.5 & 0.8660254 & 28.730 & s \\
\hline 23 & b & .0 .5 & 0.8660254 & 28.730 & s \\
\hline 24 & $p$ & 0.5 & 0.8660254 & -28.730 & $s$ \\
\hline 25 & $p$ & -0.5 & 0.8660254 & -28.730 & $s$ \\
\hline c & & & & & \\
\hline $\begin{array}{l}36 \\
37\end{array}$ & $\begin{array}{l}\rho 2 \\
\text { so }\end{array}$ & $\begin{array}{l}-42.381 \\
100.00\end{array}$ & & & $\begin{array}{l}\text { s Concrete Floor } \\
\text { s End of the world }\end{array}$ \\
\hline
\end{tabular}

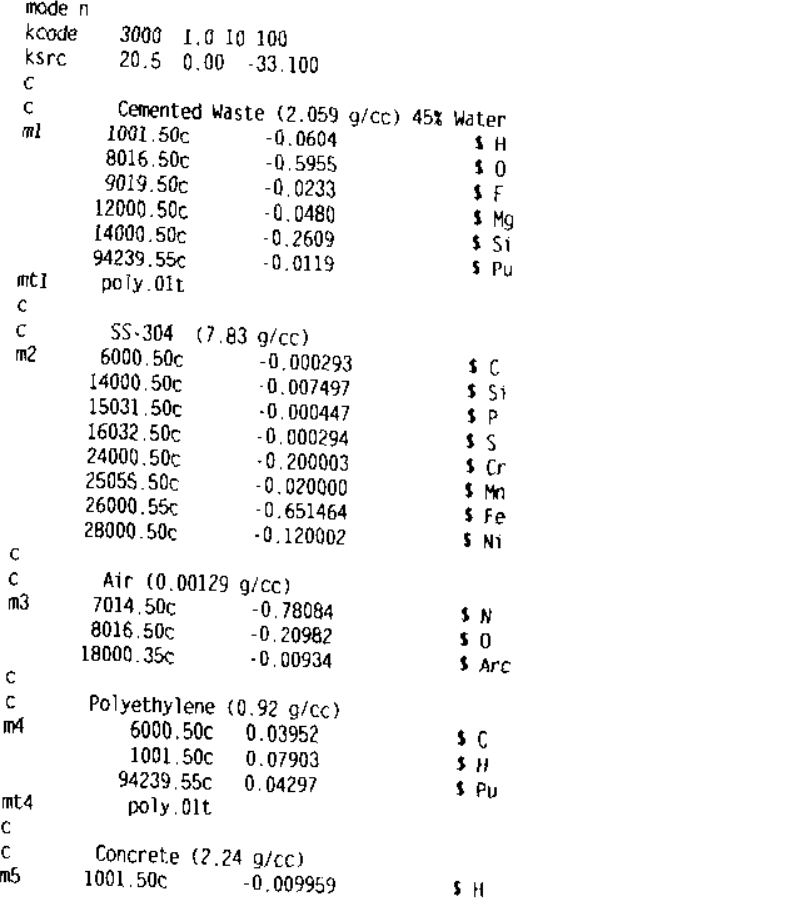


HNF-6179 Rev. 0

\begin{tabular}{|c|c|c|c|}
\hline & $6000.50 \mathrm{c}$ & 0.000979 & 30 \\
\hline & $8016.50 \mathrm{c}$ & -0.529069 & 50 \\
\hline & $11023.50 \mathrm{c}$ & -0.016003 & $5 \mathrm{Na}$ \\
\hline & $12000.50 \mathrm{c}$ & -0.002000 & $5 \mathrm{Mg}$ \\
\hline & $13027.50 \mathrm{c}$ & -0.034006 & $5 \mathrm{Al}$ \\
\hline & $14000.50 \mathrm{C}$ & -0.337036 & s si \\
\hline & $19000.50 \mathrm{C}$ & 0.012981 & s $k$ \\
\hline & $20000.50 \mathrm{c}$ & 0.043965 & $5 \mathrm{Ca}$ \\
\hline & $26000.55 c$ & -0.014002 & $s \mathrm{Fe}$ \\
\hline mt5 & poly.01t & & \\
\hline \multicolumn{3}{|c|}{ por. } & \\
\hline \multirow[t]{2}{*}{ m6 } & & & $5 \mathrm{H}$ \\
\hline & $\begin{array}{l}1001.50 \mathrm{C} \\
8016.50 \mathrm{C}\end{array}$ & $\begin{array}{l}-0.1119 \\
-0.8881\end{array}$ & 0 \\
\hline mit6 & Iwtr.01t & & s Light water \\
\hline \multicolumn{4}{|r|}{-5} \\
\hline \multirow{2}{*}{$\begin{array}{l}c \\
m 7\end{array}$} & Cellulose ( & $1.45 \mathrm{~g} / \mathrm{CC})$ & \\
\hline & $\begin{array}{l}1001.50 \mathrm{c} \\
8016.50 \mathrm{c} \\
6000.50 \mathrm{c}\end{array}$ & $\begin{array}{l}0.054311 \\
0.026949 \\
0.03233\end{array}$ & S Cellulose Reflector \\
\hline $\mathrm{mt} 7$ & poly.01t & & \\
\hline \multicolumn{4}{|c|}{ 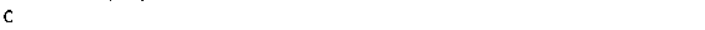 } \\
\hline \multirow{2}{*}{$\begin{array}{l}c \\
\mathrm{~m} 8\end{array}$} & Polyethyle & 1e $(0.92 \mathrm{~g} / \mathrm{Cc})$ & \\
\hline & $6000.50 \mathrm{c}$ & 0.03952 & 3 Polyethylene Reflector \\
\hline $\mathrm{mt} 8$ & $\begin{array}{l}1001.50 \mathrm{C} \\
\text { poly.01t }\end{array}$ & 0.07903 & \\
\hline \multicolumn{4}{|c|}{ poly.01t } \\
\hline \multirow{4}{*}{$\begin{array}{l}\mathrm{c} \\
\mathrm{m} 9\end{array}$} & Water $(1.02$ & $g /(c)$ & \\
\hline & $1001.50 \mathrm{C}$ & -0.1097 & $\$ \mathrm{H}$ \\
\hline & $8016.50 \mathrm{C}$ & $\cdot 0.8703$ & $\$ 0$ \\
\hline & $94239.55 \mathrm{C}$ & -0.0200 & s $\mathrm{Pu}$ \\
\hline $\mathrm{mt} 9$ & Iwtr.01t & & s Light water \\
\hline c & & & \\
\hline totnu & & & \\
\hline $\begin{array}{l}\text { ctme } \\
\text { print }\end{array}$ & 480 & & \\
\hline
\end{tabular}

odrum_c.inp

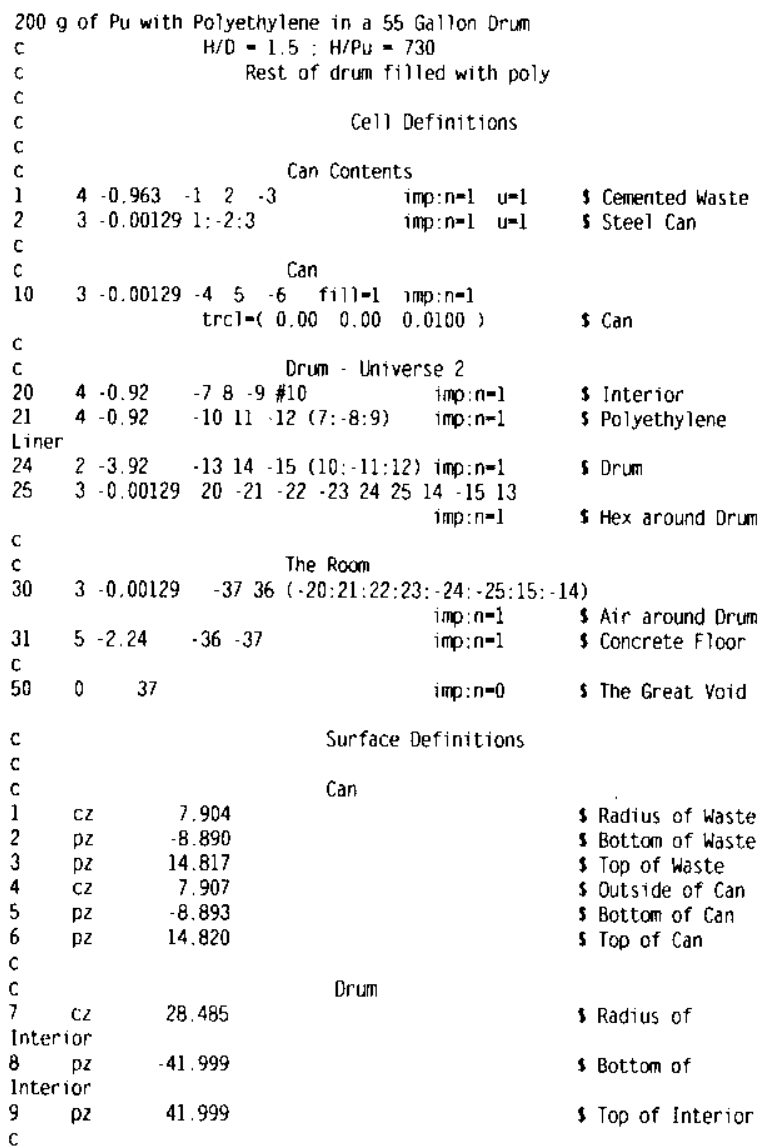


HNF-6179 Rev. 0

totnu
ctme
print

ovbtch_botlp.inp

Three Billets in a Non-pipe 55 Gallon Drum

$c$ three overpacked Pu billets at the

filled with poly

silicon replaces calcium

Cell Definitions

$\begin{array}{lllllll}\text { C Can Contents - Universe } 1 & \\ \text { imp: } n=1 \quad u=1 & 1 \cdot 1.04 & -1 & 2 & -3 & \end{array}$

$\begin{array}{llll}1 \cdot 1.04 & -12^{-3} & \text { imp:n=1 } u=1 & \text { s Cemented Waste } \\ 3 \cdot 0.00129 & 1:-2: 3^{i m p: n=1} u=1 & \text { s steel Can }\end{array}$

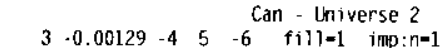
$\operatorname{trcl}=\left(\begin{array}{ccc}8.150 & 0.00 & -33.100\end{array}\right)$

11 like 10 but $\operatorname{trcl}=\left(\begin{array}{cccc}20.250 & 7.015 & -33.100) & \text { \$ Can } 1 \\ & \text { \$ Can } 2\end{array}\right.$

12 like 10 but $\operatorname{trcl} 1-(20.250-7.015-33.100)$ s Can 3

c $\quad$ Orum - Universe 2

$204-0.92 \quad-78-9 \$ 10 \$ 11 \# 12$ imp:n=1 s Interior

$21 \quad 4 \cdot 0.92 \quad-1011-12(7:-8: 9)$ imp:n-1 s Polyethylene

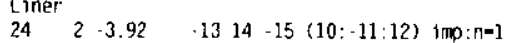

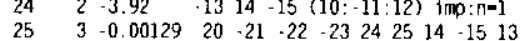

imp: $n=1$

\$ Drum

S Hex around Drum

c $\quad$ The Room

$303-0.00129-3736(-20: 21: 22: 23:-24:-25: 15:-14)$

$\begin{array}{lrrrrrr}31 & 5 & -2.24 & -36 & -37 & \begin{array}{l}\text { imp:n=1 } \\ \text { imp:n=1 }\end{array} & \text {; Air around Drum } \\ c & & & & \text { imp:n=te Floor } & \text { s The Great void }\end{array}$

c
c
1
2
3
4
5
6
C
c
7
Inter
8
Inter

C2 $\quad 6.985$

6.985
-8.890

8.890

6.988

.8 .893

Surface Definitions

Billets

p2 $\quad 8.893$

28.485

Drum

$-41.999$

41.999

28.575

28.575
-42.227

42.227

28.727

$-42.379$

42.379

42.380

Hexagonal Surfaces $(A x+B y+C z-D)$

-28.729
28.729

28.729

$\begin{array}{cc}A & B \\ 0.5 & 0.8660254 \\ 0.5 & 0.8660254 \\ 0.5 & 0.8660254\end{array}$

$\begin{array}{rr}0.5 & 0.8660254 \\ -0.5 & 0.8660254\end{array}$

-42.381
100.00

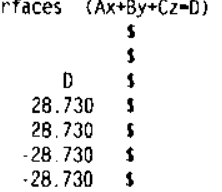

s Concrete Floor

s End of the World 5 8ottom of Waste 5 Top of Waste

$\checkmark$ Outside of Can

Bottom of Can

5 Radius of

5 Bottom of

s Top of Interior

5 Radius of Liner 5 Bottom of Liner 5 Top of Liner

5 Radius of Drum

Bottom of Drum

s Top of Drum

Top above Drum
5 Radius of waste

$\begin{array}{lllll}\text { kcode } & 3000 & 1.0 & 10 & 100 \\ \text { ksrc } & 8.150 & 0.00 & -33.100\end{array}$

c Cemented Waste $(1.041 \mathrm{~g} / \mathrm{cc}) 1008$ water

$\begin{array}{rrr}1001.50 \mathrm{C} & -0.1086 & \text { S H } \\ 8016.50 \mathrm{C} & -0.8623 & \text { \$ } 0 \\ 94239.55 \mathrm{C} & -0.0289 & \text { S Pu }\end{array}$

$\mathrm{mt} 1$ poly.01t

$\$ \mathrm{Pu}$

c $\quad 55.304 \quad(7.83 \mathrm{~g} / \mathrm{CC})$

$6000.50 \mathrm{C} \quad-0.000293$

s C

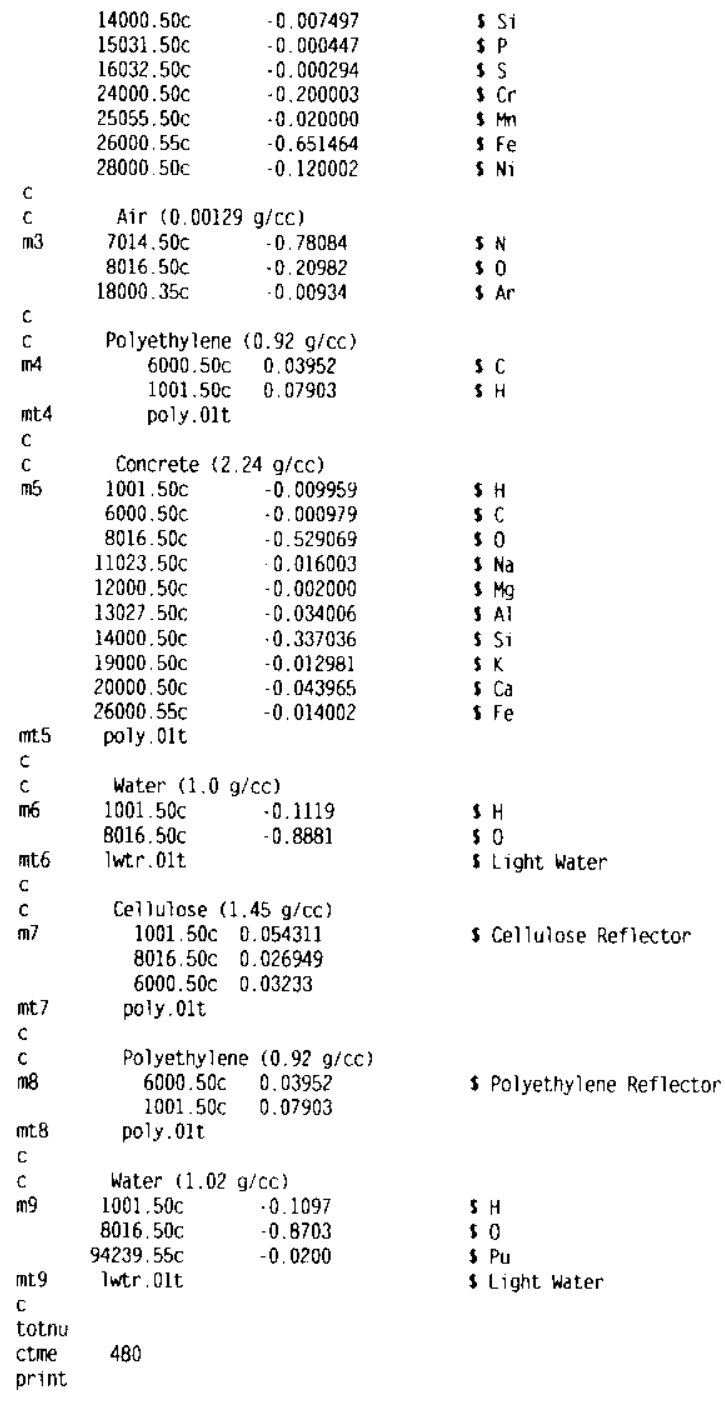

ovbtch_ref2p.inp

Three Billets in a Non-pipe 55 Gallon Drum

three Pu billets at the bottom of a $400 \mathrm{~g} / \mathrm{drum}$

one relecting boundary (two drums)-Poly

Cell Definitions

$\begin{array}{lllll}1-1.04 & -1 & 2 & -3 & \text { Can Contents - Universe } 1 \\ \text { imp: } n=1 & u=1\end{array}$ s Cemented Waste $3-0.001291:-2: 3^{-3} \quad$ imo: $n=1 \quad u=1$ steel Can

$\begin{array}{lllll}3-0.00129 & -4 & 5 & -6 & \text { Can - Universe } 2 \\ \text { fill }=1 \text { imp:n=1 }\end{array}$ trcl-( $\left.8.1500 .00^{1 m p: n=1}-33,100\right) \quad$ s Can 1

like 10 but $\operatorname{trcl}=(20.250 \quad 7.015-33.100)$ s Can 2

like 10 but $\operatorname{trcl}=(20.250 \cdot 7.015-33.100) \quad$ s Can 3

$4-0.92 \quad-78-9 \$ 10 \$ 11 \$ 12$ imp:n=1 s interior

$21 \quad 4 \cdot 0.92 \quad-1011-12(7 ;-8: 9) \quad$ imp:n=1 s Polyethylene

$24 \quad 2-3.92-1314-15(10:-11: 12)$ imp:n-1 S Drum

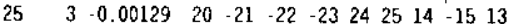

imp:n=i \& Hex around Drum

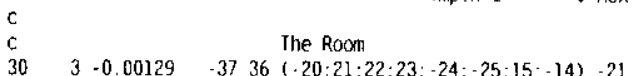

$31 \quad 5 \cdot 2.24 \quad-36-37-21 \quad$ imp:n=1 \& Air around Drum

31 $5 \cdot 2.24 \quad-36-37-21 \quad$ imp: $n=1 \quad$ s Concrete Floor 
HNF-6179 Rev. 0

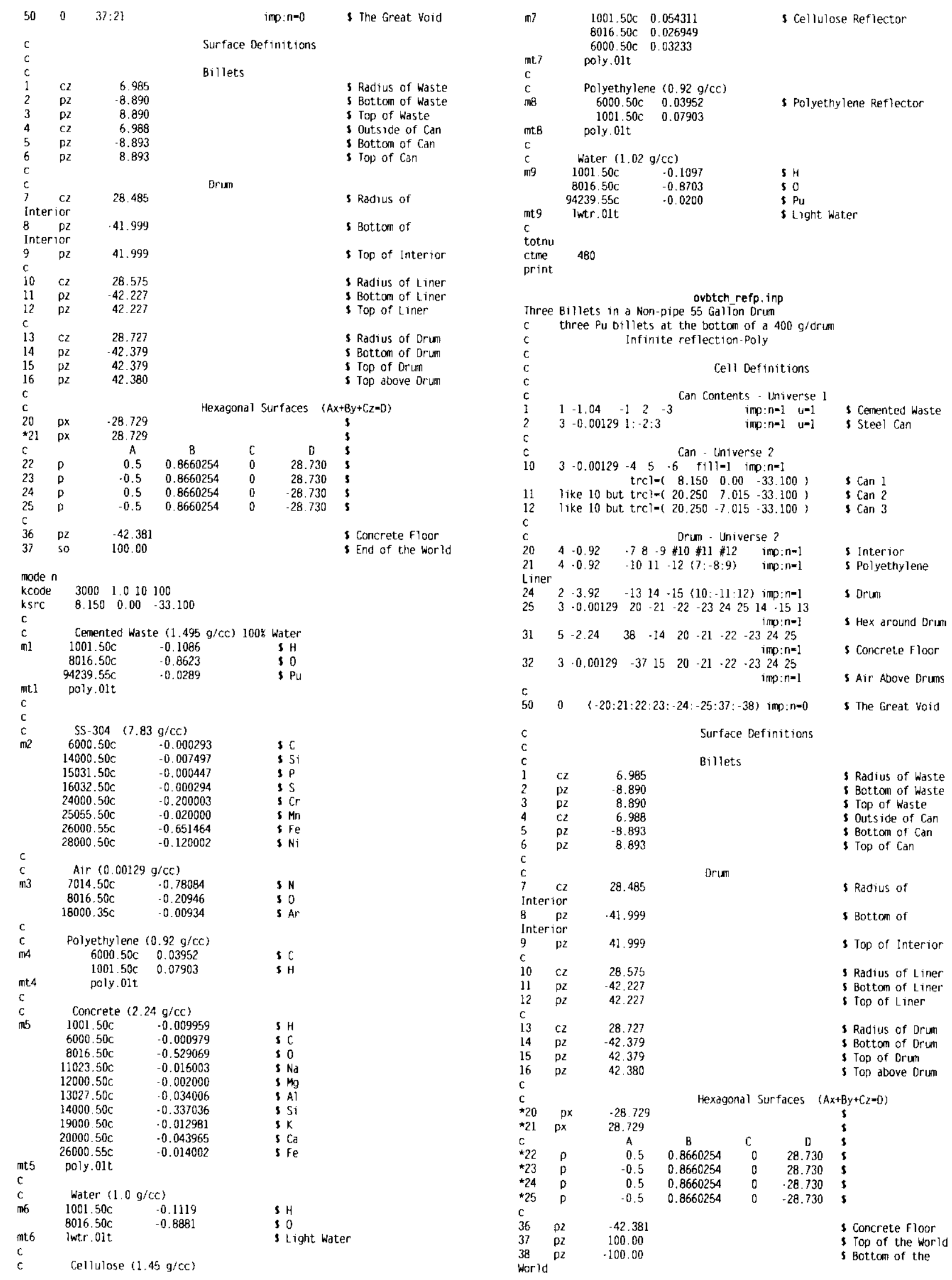


HNF-6179 Rev. 0

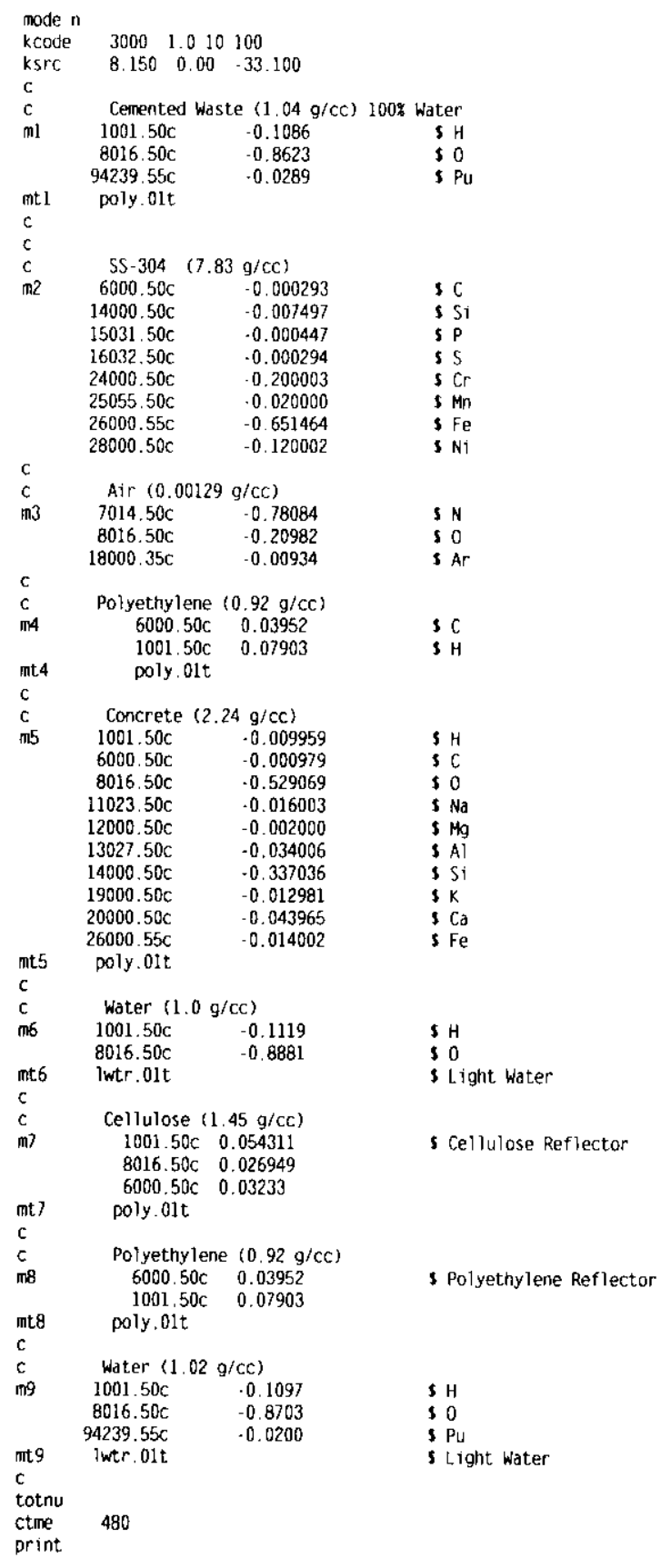

ovbtchrefrotp.inp

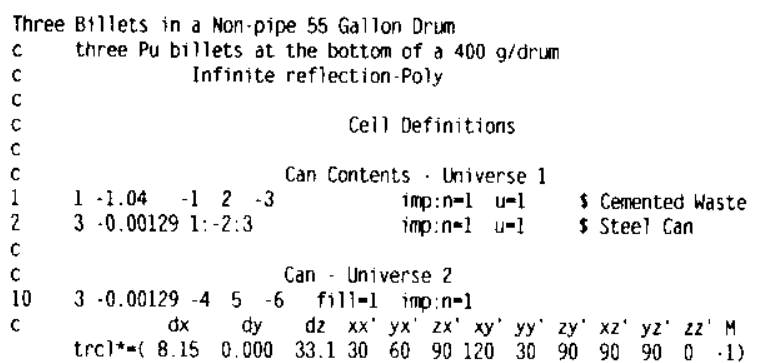

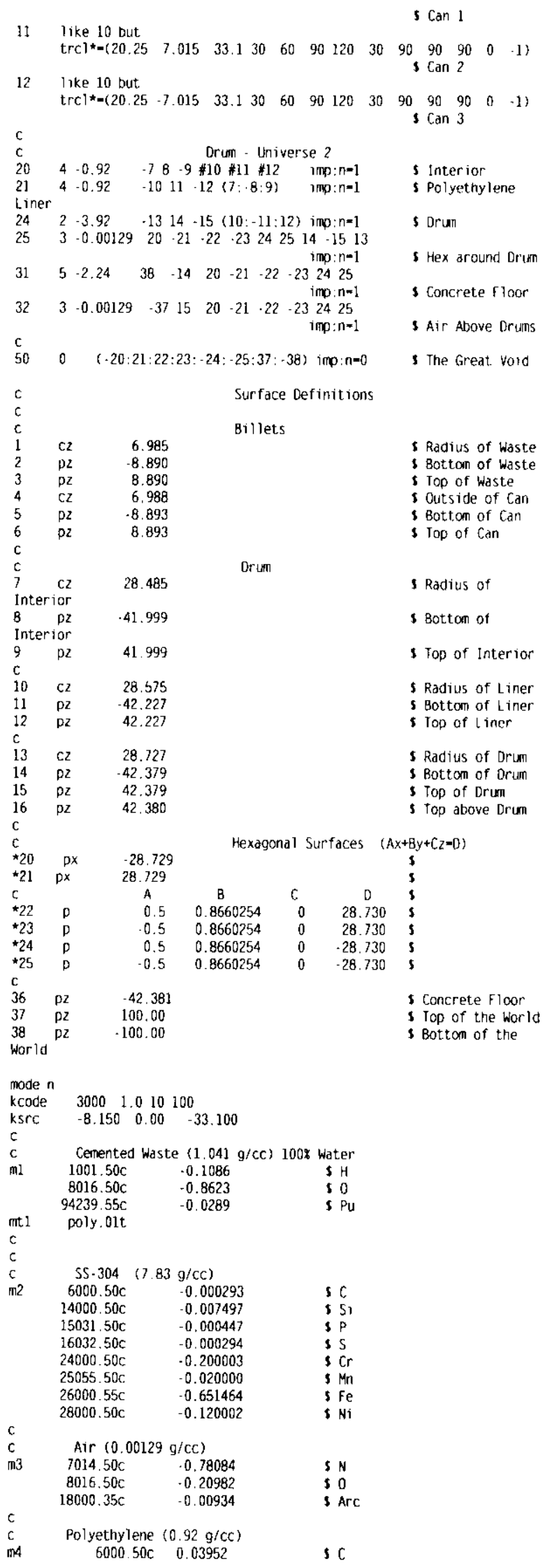


HNF-6179 Rev. 0

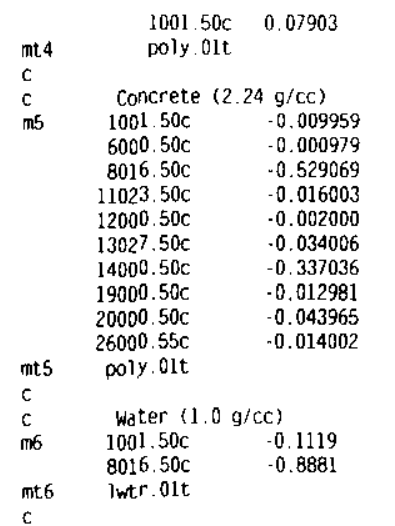

\$ H
$5 \mathrm{H}$
$5 \mathrm{C}$
$5 \mathrm{O}$
$5 \mathrm{Na}$
$5 \mathrm{Mg}$
$5 \mathrm{Al}$
$5 \mathrm{Si}$
$5 \mathrm{~K}$
$5 \mathrm{Ca}$
$5 \mathrm{Fe}$

$5 \mathrm{H}$
\$ $\mathrm{O}$
Light water

\begin{tabular}{|c|c|c|}
\hline c & Cellulose (1 & $1.45 \mathrm{~g} / \mathrm{CC})$ \\
\hline$m 7$ & $\begin{array}{l}1001.50 c \\
8016.50 c \\
6000.50 c\end{array}$ & $\begin{array}{l}0.054311 \\
0.026949 \\
0.03233\end{array}$ \\
\hline $\begin{array}{l}m ! 7 \\
c\end{array}$ & \multicolumn{2}{|c|}{ poly.01t } \\
\hline$c$ & \multirow{2}{*}{\multicolumn{2}{|c|}{$\begin{array}{cl}\text { Polyethylene }(0.92 \mathrm{~g} / \mathrm{cc}) \\
6000.50 \mathrm{C} & 0.03952 \\
1001.50 \mathrm{c} & 0.07903\end{array}$}} \\
\hline m8 & & \\
\hline $\mathrm{mt} 8$ & \multirow{2}{*}{\multicolumn{2}{|c|}{ poly.01t }} \\
\hline $\mathrm{C}$ & & \\
\hline c & \multicolumn{2}{|c|}{ Water $(1.02 \mathrm{~g} / \mathrm{cc})$} \\
\hline $\ln 9$ & $\begin{array}{r}1001.50 \mathrm{c} \\
8016.50 \mathrm{C} \\
94239.55 \mathrm{C}\end{array}$ & $\begin{array}{l}-0.1097 \\
-0.8703 \\
-0.0200\end{array}$ \\
\hline mt9 & $\begin{array}{l}94239.536 \\
\text { iwtr.01t }\end{array}$ & \\
\hline $\begin{array}{l}\text { c } \\
\text { totnu } \\
\text { ctme }\end{array}$ & 480 & \\
\hline int & & \\
\hline
\end{tabular}

s Cellulose Reflector

S Polyethylene Reflector

$5 \mathrm{H}$

$5 \mathrm{Pu}$

$s$ Light water 
HNF-6179 Rev. 0

This page intentionally left blank. 
HNF-6179 Rev. 0

APPENDIX D - PRELIMINARY HAZARDS ANALYSIS

D-1 
HNF-6179 Rev. 0

This page intentionally left blank.

D-2 
HNF-6179 Rev. 0

\section{List of Terms}

$\begin{array}{ll}\text { AIChE } & \text { American Institute of Chemical Engineers } \\ \text { CSER } & \text { Criticality Safety Evaluation Report } \\ \text { CWC } & \text { Central Waste Complex } \\ \text { GB } & \text { glovebox } \\ \text { ID } & \text { Identification Number } \\ \text { NC } & \text { no controls } \\ \text { PHA } & \text { Preliminary Hazards Analysis }\end{array}$

\section{List of Definitions}

Container - Any vessel that is in a position to hold solids or liquids (e.g., a pan turned upside down is not a container).

Contingency - A possible but unlikely change in a condition/control important to the nuclear criticality safety of a fissionable material operation that would, if it occurred, reduce the number of barriers (either administrative or physical) that are intended to prevent an accidental nuclear criticality. 
HNF-6179 Rev. 0

This page intentionally left blank. 


\section{PLUTONIUM RESIDUE IN 55-GALLON DRUM CRITICALITY PRELIMINARY HAZARDS ANALYSIS}

\subsection{PURPOSE}

The purpose of this Preliminary Hazard Analysis (PHA) is to identify potential events and conditions that could lead to a nuclear criticality in the cemented Sand, Slag, and Crucible (SS\&C), ash, oxide or other miscellaneous residues stored within 55-gallon drums at the Plutonium Finishing Plant (PFP). The results of this PHA will be used for input into the Plutonium Residue in 55-Gallon Drum Criticality Safety Evaluation Report (CSER).

This PHA was conducted in two stages. At the beginning of CSER preparation, drum loading in Building 234-5Z was assumed to be in Room 235B with storage in Rooms 172 and 192D as described in Section 2.0. The areas of the facility assessed at that meeting are illustrated in Figure 1 with the results summarized in Table A1. After the CSER was completed, a second PHA meeting was conducted to identify to what extent the CSER would apply to loading drums and storing them in the balance of Building 234-5Z. In addition, the dropped billet and drum tipping/rolling events were reevaluated as likely rather than unlikely events. Results of the second PHA meeting are described in Section 6.0.

\subsection{PROCESS DESCRIPTION USED IN CSER PREPARATION}

The cementation process will take place within the Plutonium Finishing Plant (PFP). Billet cans of cemented Sand, Slag, and Crucible (SS\&C) scrap are removed from the HA-28 conveyor belt in room $235 \mathrm{~B}$ through a 12 -inch sealout port, individually bagged, and loaded into 55 -gallon steel drums with rigid polyethylene liners. The billet cans bagged out of HA-28 may also be placed into 55-gallon drums containing pipe overpack containers (POC). One drum at a time will be loaded within a containment structure. The drums from either cementation or other processing activities will be sealed, loaded onto a transfer cart, and moved to a temporary storage location within the PFP to await shipment to offsite disposal. The transfer cart will maintain at least 18 inches of clearance from any other fissile material. Relief from the 18-inch rule may be needed for the passage between HA-20MB, HA-21I and the 235B stairway.

If the temporary storage location is room $192 \mathrm{D}$, the drum will move down room $235 \mathrm{~B}$, through rooms $235 \mathrm{C}$ and $235 \mathrm{D}$, through the room 193 airlock, into room 192, and from there into room 192D. It will be removed through corridor 192 and door 133 to the West Pad for loading onto a transport truck for shipment to the Central Waste Complex (CWC) (see Figure 1). If the temporary storage location is room 172 , the drum will move down room $235 \mathrm{~B}$, through room $235 \mathrm{C}$ and room 245 to Corridor 6 , and into room 172 . It will be removed through corridors 6,7 , and 7A and door 131 to the West Pad for loading onto a transport truck for shipment to the CWC.

A PHA is a hazards identification/evaluation technique derived from the U.S. Military Standard System Safety Program Requirements (MIL-STD-882). A PHA is a structured 
discovery process involving a multi-disciplinary team and focuses on the hazardous materials and major process areas of a facility. The results of the PHA are recorded using a tabular format. Because of its military heritage, the PHA technique is useful for reviewing process areas where energy can be released in an uncontrolled manner. In general, the PHA formulates a list of hazards and hazardous situations by considering the following process characteristics:

- Raw materials, intermediate and final products, and their reactivity

- Plant equipment

- Facility layout

- Operating environment

- Operational activities (testing, maintenance, etc.)

- Interfaces among system components.

The American Institute of Chemical Engineers (AIChE) recognizes the PHA process as a creditable method of hazard evaluation. AIChE describes this process in their publication titled "Guidelines for Hazard Evaluation Procedures" (AIChE 1992).

The depth of a PHA is directly related to the experience and knowledge of the participants. A short resume of each team member is included (Section 3.0) to document the experience and knowledge of the PHA team.

\subsection{PHA EVALUATION STRUCTURE}

The hazardous condition of concern for this PHA is a nuclear criticality. Criticality events are prevented by establishing limits for specific parameters. These parameters are referred to as controlled parameters. This PHA was structured to address the following controlled parameters:

Mass

Volume

Geometry

Moderation

Reflection

Interaction

Enrichment

Density

Concentration

Poisons

The process occurring involves loading the cans containing the SSC scrap into a 55gallon drum, transferring the drum to a storage location, and transferring the drum to the truck loading dock. It is conservatively assumed that all cans are unconstrained in position inside the drum. The PHA was structured as follows to evaluate the controlled parameters associated with process steps and conditions: 
1. Processes Occurring in Containment Envelope

- Removal of billet can from conveyor

- Billet can seal out

- Drum loading

2. Processes Occurring Outside Containment Envelope

- Move drum to storage

- Drum storage

- Move drum to loading dock

External Events

Fire

Flood

Seismic

High winds

\subsection{PHA TABLE DESCRIPTION}

The PHA table (Table A1) was structured to ensure a systematic and thorough evaluation of the controlled parameters. The PHA table captured the following information:

ID: Item identification used to record a unique identifier for the hazardous condition.

Process Condition or Step: The process step or condition being evaluated for potential situations which can result in changes in the controlled parameters that could cause a criticality during cementation drum loading.

Event and Detailed Causes: Specific events and causes that have the potential to result in a criticality. This column is used to capture details that may be important in the analysis that will define the controls to prevent a criticality.

Controlled Parameter: Parameters that are controlled to prevent the occurrence of criticality events. Ten parameters are used: mass, volume, moderation, interaction, reflection, geometry, enrichment, density, concentration, and poisons.

Engineered Safety Features: Hardware items that are identified by the PHA team that have the potential to mitigate or prevent the hazardous condition of concern.

Administrative Safety Features: Administrative controls such as facility worker training and safety procedures identified by the PHA team that have the potential to mitigate or prevent the hazardous condition. 
Freq Cat NC: Frequency Category, No controls - The frequency ranking is a qualitative estimate of whether the hazardous condition is considered credible (C) or not credible (NC). Conditions considered NC will not be evaluated further. Credible conditions considered likely (L) are considered in the base case analysis. Credible conditions considered unlikely $(\mathrm{U})$ are considered as contingencies to the base case.

Remarks: Miscellaneous observations or clarifying comments provided for a given item. This column is also used to capture criticality analysis requirement decisions of the PHA team.

\subsection{PHA RESULTS}

The results of this PHA are in the form of information to be considered as part of the Criticality Safety Evaluation Report (CSER). The raw data are presented in Table A1. The information contained in the PHA table is used to ensure that the CSER analysis addresses the appropriate conditions that may contribute to a criticality in the HC-20MB glovebox. The following assumptions that are important to the CSER analysis were extracted from Table A1 and discussions during the PHA.

\section{Plutonium Residue in 55-Gallon Drum PHA Assumptions and Dispositions Derived from the PHA:}

1. Analysis scope covers the following process steps: seal out billet, load billet in drum, move drum to storage location, and store the drum. SARP covers shipment of drum to CWC.

2. CSER 96-027 is to be replaced via this analysis.

3. This analysis assumes that loading more than one drum at a time with cemented billets is not credible, based on the anticipated size of the containment enclosure.

4. Each billet (2.7 L, 5.5" dia x 7" high) is sealed out one at a time in a plastic bag. Sealout of more than one billet per bag is a contingency considered in ID \#1 of Table A1.

5. Drums will be transported to Room 192D or other location for storage (see Section 2.0 of this Appendix). This analysis is intended to be flexible enough to accommodate several possible storage locations.

6. The mass limit evaluated in this analysis is a maximum of $200 \mathrm{~g} \mathrm{Pu}$ or fissile equivalent for the unconstrained drum or 6-inch or 12-inch pipe overpack.

7. The current minimum distance allowed between the edge of the container and any other fissile material over $100 \mathrm{~g}$ is 18 inches during transit. Drums passing by HA-20MB and HA21 I may require an exception to the 18 -inch rule. The analysis will evaluate this condition.

8. This analysis assumes that drums will pass by HA-23S at a minimum of 3 feet. The analysis will evaluate closer distances as a contingency. 
9. Preferred storage of drums in $192 \mathrm{D}$ is $2 \mathrm{x}$ infinite arrays, 1 high, butted up against concrete walls and directly on floor. The analysis assumes an infinite array ( $1 \mathrm{high}$ ) in the horizontal plane. The drums are to be arranged in a square array, but the analysis will consider arrangement in a hexagonal array.

10. Drums weigh from 120 to $300 \mathrm{lbs}$. The analysis will consider the weight of the drum and wagon.

11. Intermixing of other types of waste drums in an infinite array is considered likely and will be evaluated in the analysis. This condition is listed in ID \#16 of Table A1.

12. The analysis assumes unrestricted moderation.

13. The analysis assumes that drums have heads and bolt rings on during transit and storage. Transporting an open drum is considered "unlikely" in ID \#15 of Table A1 and will be evaluated as a contingency.

14. The analysis assumes that only one drum will be transported at a time.

15. The drum will be latched to a carrier for moving. Failure of the latch is considered credible but unlikely. It is not credible for a tipped and rolling drum to damage a "wagon" or to roll under a glovebox. See ID \#13 of Table A1.

16. The analysis assumes that the drum array can only be crushed vertically. See ID \#18 of Table A1.

17. Cans may be loaded either in drums with a center pipe, or in unconstrained drums.

18. In addition to cemented SSC scrap, the drum may be loaded with cans containing uncemented ash and/or oxide.

\subsection{EXTENSION OF PHA TO LOAD AND STORE DRUMS IN OTHER ROOMS OF BUILDING 234-5Z}

As a second stage to the PHA, the team members listed in Section 7.0 reconvened to evaluate the applicability of the CSER, based upon the hazards review of the process described in Section 2.0, to other rooms in the PFP Building 234-5Z. PFP operations would like to extend the CSER analysis for loading and storing drums for processing activities other than cementation in as much of the building as possible.

The worst mass overload analyzed was $400 \mathrm{~g}$ plutonium with unrestricted moderation. Therefore, areas of the building where an overbatch contingency greater than $400 \mathrm{~g}$ was credible need to be excluded. Because the scrap and billet containers are larger and physically different 
from those containing metal or oxide, it was concluded that operator training and procedure would preclude accidental loading of greater mass containers into these drums.

In a seismic event, either end of the plutonium nitrate transfer piping from Room 227 to Room $230 \mathrm{C}$ could be a source of liquid fissile material not analyzed in this CSER. The pipe conduit is seismically qualified for the rest of its length, so the drums need to be excluded from these rooms at the source and destination of the piping but not in between. It is not possible to get plutonium nitrate in these drums elsewhere in 234-5Z. Open drums are not stored at the PFP, which precludes an accidental accumulation of other forms of fissile material in these drums. The PHA team concludes that it is not credible to get more than $400 \mathrm{~g}$ of plutonium into these drums anywhere else in Building 234-5Z.

The worst interaction effect analyzed was a five-position wagon loaded with $2.5 \mathrm{~kg}$ plutonium as metal or $4.5 \mathrm{~kg}$ plutonium as oxide in each position. Vaults, with their greater fissile mass loading, are outside of this analysis and are excluded from allowed locations for loading and storing these drums. The PHA team reviewed the fissile mass inventory of gloveboxes, transfer containers, and storage arrays for possible interaction with these drums throughout the balance of Building 234-5Z. The team concluded that the fully-loaded wagon, already analyzed in the CSER, was the worst interaction event. Interaction with glovebox contents, drums with plutonium nitrate, or isolated transport containers are bounded by the wagon/drum analysis.

The CSER includes a single drum/wagon interaction as a likely event in one of the base cases. The dropped billet and drum tipping/rolling events are bounded by the wagon interaction and there is no need to consider them as contingencies. Initially, the PHA team concluded that a dropped billet and drum tipping/rolling were unlikely events. However, upon reconsideration, it was agreed that these could be considered likely, that they are bounded by the base case, and that they could be removed from the contingency table in the CSER. Table Al is therefore changed to indicate dropped billets and drum tipping/rolling as likely. 


\subsection{PHA TEAM MEMBER BIOGRAPHIES}

David J. Braun, Fluor Federal Services, Inc., Engineer, Safety Analysis and Risk Assessment. Mr. Braun has over 21 years experience at Hanford working primarily in Safety Analysis. His experience includes Probabilistic Risk Analysis of nuclear reactors, tank farm activities, and hazards identification and evaluation of various Hanford site operations and facilities. He was one of the lead engineers on the hazards identification and evaluation team for the current TWRS BIO and FSAR. He has facilitated numerous PHA and HAZOP studies for projects at the Hanford site. Mr. Braun has a Master of Science Degree in Mechanical Engineering from the University of California at Davis. He is a registered Professional Engineer in Mechanical Engineering (Washington State).

Lane Cunningham, Parsons Engineering, Project Engineer. Mr. Cunningham has 9 years experience working at the Hanford site. The last 7 years were spent working on stabilization and baseline projects for the Plutonium Finishing Plant (PFP). While a direct employee at the PFP, he served as a shift technical adviser and held various cognizant engineer positions in support of stabilization activities, including cementation. His primary responsibilities included installation, start-up, and operational support for the Thermal Stabilization process, cementation process, Vertical Denitration process, and the Low Level Waste Treatment Facility (LLWTF). He has both undergraduate and master degrees in chemical engineering from the University of North Dakota. Mr. Cunningham is currently serving as the Acting Project Manger for the cementation project.

LaPriel Dayley, Project Enhancement Corporation, Project Engineer. Ms. Dayley has 15 years of experience working at the Hanford Site. She spent about 4 years as technical support for $\mathrm{N}$ Reactor and 7 years as Process Engineer at PFP. She was the Cementation process cog engineer for initial process start up in 1996. Her experience includes specifying equipment, acting as PIC for equipment installation, developing procedures and technical basis documents and shift technical support. Ms. Dayley has a Bachelor of Science degree in Chemical Engineering from the University of Washington.

Ken Dobbin, Fluor Federal Services, Inc, Criticality Safety Engineer. Mr. Dobbin has 25 years experience as a nuclear engineer, 20 of these years analyzing reactor physics and fuel management and 5 years in criticality safety. He is qualified as a Criticality Safety Engineer at the Plutonium Finishing Plant and has 20 months experience with PFP systems. During his PFP tenure, he contributed criticality safety expertise for the successful completion of an Operational Readiness Review to resume thermal stabilization of plutonium. Mr. Dobbin has both undergraduate and masters degrees in nuclear engineering.

David G. Erickson, Fluor Federal Services, Criticality Safety Engineer. Mr. Erickson has a B. S. in Physics, and has been at the Hanford Site for 16 years performing analysis in the fields of reactor physics and 13 years in criticality safety. He is familiar with the codes used in criticality analysis and has performed analysis and reviews of other PFP operations. He is a qualified Criticality Safety Specialist with FFS. 
Harvey J. Goldberg, Fluor Federal Services, Inc., Engineer. Dr. Goldberg has 21 years at the Hanford Site. His experience has been in PFP, WRAP, BWIP, HWVP, and packaging and shipping. This experience has been in the field of safety concerned with shielding, criticality, operational procedures, source development, and facility siting, design, and regulatory approval. Dr. Goldberg has a B. S. from the University of Rhode Island, and an M. S. and Ph. D. from Kansas State University as well as certification from the American Academy of Health Physics.

Brit E. Hey, Fluor Federal Services, Inc., Safety Analysis and Risk Assessment. Mr. Brit Hey has over 18 years of combined engineering and management experience in the commercial and DOE nuclear industries. For the last 10 years Mr. Hey has performed safety analysis for numerous Hanford facilities and activities including tank farms, the waste encapsulation and storage facility, B-Plant, and the plutonium finishing plant. Mr. Hey has successfully completed the process hazard analysis leader training course offered by the ABS Group, Inc. Mr. Hey has both undergraduate and masters degrees in nuclear engineering from North Carolina State University.

James R. Hilliard, Fluor Hanford, PFP Solid Waste Operations, Team Leader. Mr. Hilliard has 16 years of waste management and fissile material handling experience at the Hanford Site including nuclear fuel fabrication, reactor operations, hazardous and radioactive waste packaging and shipping. He was the PUREX Solid Waste Cognizant Engineer and Team Leader for the PUREX Waste Team through the PUREX deactivation. Jim currently is the Team Leader for the PFP Solid Waste Operations Team, which manages all containerized wastes at the PFP.

James R. Hilliard, Fluor Hanford, PFP Solid Waste Operations, Team Leader. Mr. Hilliard has 16 years of waste management and fissile material handling experience at the Hanford Site including nuclear fuel fabrication, reactor operations, hazardous and radioactive waste packaging and shipping. He was the PUREX Solid Waste Cognizant Engineer and Team Leader for the PUREX Waste Team through the PUREX deactivation. Jim currently is the Team Leader for the PFP Solid Waste Operations Team, which manages all containerized wastes at the PFP.

Robert F. Richard, Fluor Federal Services, Inc., Criticality Safety Engineer. B. S. Nuclear Engineering. Thirteen years experience at Hanford performing nuclear engineering analyses in fuel management, reactor physics, radiation shielding, and seven years in criticality safety.

Maria E. Shaw, B\&W Federal Services, PFP Nuclear Criticality Safety Representative, Nuclear Engineer. Ms. Shaw has worked as an engineer since 1991 and has been at the Hanford site since 1998. She has worked at the Plutonium Finishing Plant as a criticality safety representative and is plant certified as a Criticality Safety Representative. She has worked on the PFP thermal stabilization project. Ms. Shaw holds a B. S. in Math from Idaho State and an M. S. in Chemistry from Univ. of Idaho. Prior to Hanford, she worked in the criticality safety field at Idaho National Engineering Laboratory and at the Naval Nuclear Fuel Facility (Lynchburg, VA.)

Blaise S. Mo, Fluor Hanford Company, Criticality Safety Representative for PFP. B. S. Physics and M. S. Environmental Science and Engineering. Over seventeen years experience in various 
assignments at the Rocky Flats Environmental Technology Site, including eleven years as a criticality safety engineer and six years in managing analytical laboratories, training programs, and facility operations. Recently assigned as a CSR at PFP.

Brian D. Skeels, Fluor Hanford Company, PFP Stabilization Operations, Thermal Stabilization Support Team Leader. Mr. Skeels has 15 years experience at the Hanford Site. His experience has been at 100-KE/KW basins, $100 \mathrm{~N}$ and PFP. Mr. Skeels has been at PFP for 12 years with 9 years as a First Line Supervisor/Manager. Mr. Skeels is currently the Team Leader for the Thermal Stabilization Support Team (TSST). Prior to being assigned as the TSST Team Leader, Mr. Skeels was a Shift Manager in charge of both the Cementation and Thermal Stabilization Processes during their operating campaigns. Mr. Skeels is currently qualified on the Cementation process as it was operated during the last campaign.

Hans Toffer, Fluor Federal Services, Inc., Criticality and Shielding. Managed technical organizations and projects with emphasis on criticality safety, reactor physics and nuclear engineering. Background includes: Criticality safety of plutonium, high enriched uranium, low enriched uranium processing, storage and handling, thermal reactor core analysis and measurements, neutronic, ultrasonic and vibration measurement technology development, isotope production, reactor design analysis, computer code software quality assurance, organizing national technical meetings and participating in American National Standards development. Responsibilities included: Technical management and direction technical team leadership, coordination of multi-discretionary teams, served as classification officer, chairman of American National Standards working group ANS 8.21, chaired and organized national/international technical meetings, and served on special assessment teams at different Department of Energy contractor sites.

James C. Williams, Fluor Federal Services, Inc., Safety Analysis and Risk Assessment. Mr. Williams has fourteen years of experience in the nuclear industry, including five years at the Hanford site. At Hanford, he has worked on waste tank characterization, vadose zone characterization, and safety analysis for tank farms operations. Mr. Williams has a bachelor of science degree in nuclear engineering from the Pennsylvania State University. 
HNF-6179 Rev. 0

\subsection{References}

AIChE, 1992, Guidelines for Hazard Evaluation Procedures, American Institute of Chemical Engineers, New York, New York.

MIL-STD-882B, 1977, System Safety Program Requirements, Department of Defense, Washington, DC 


\section{HNF-6179 Rev. 0}

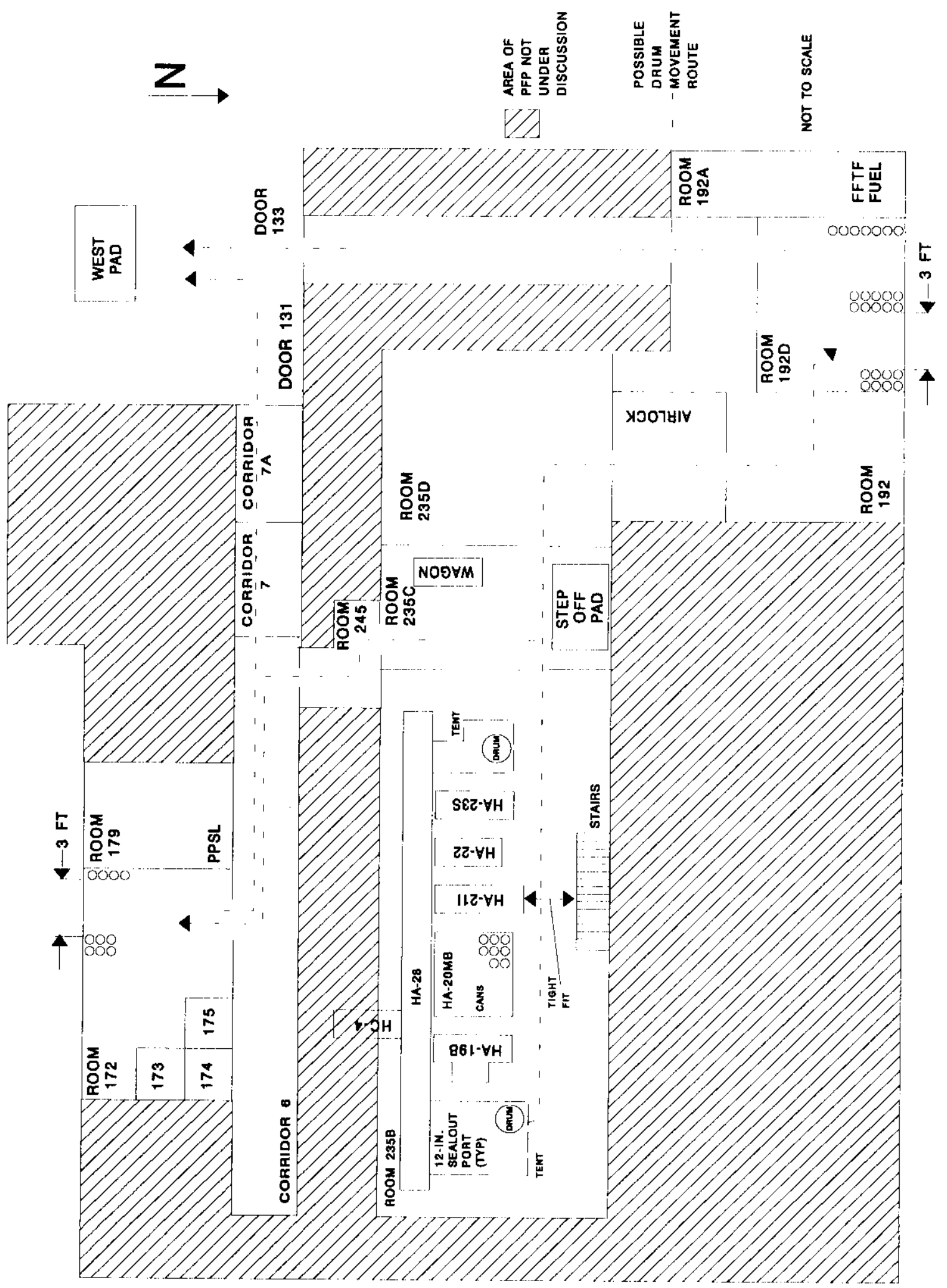

D-15 
HNF-6179 Rev. 0

\begin{tabular}{|c|c|c|c|c|c|c|c|c|c|c|c|c|c|c|c|c|}
\hline 畜 & 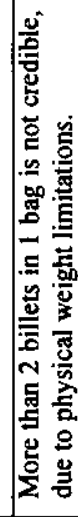 & 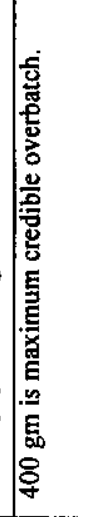 & 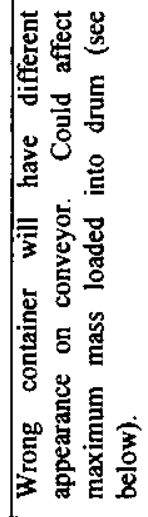 & 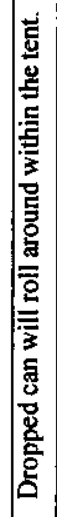 & 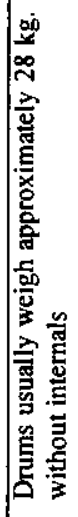 & $\mid$ & 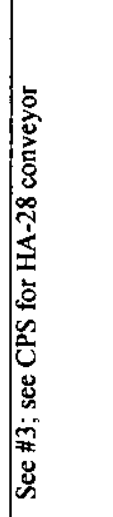 & 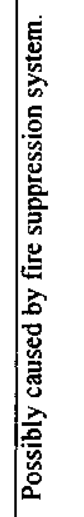 & : & 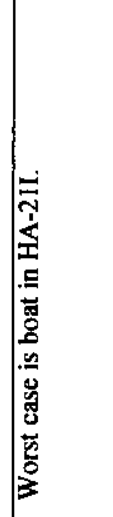 & 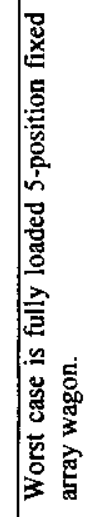 & 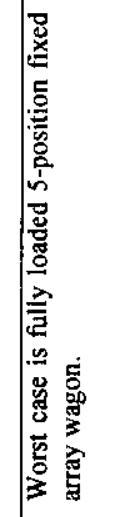 & 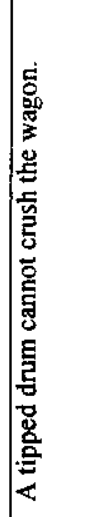 & 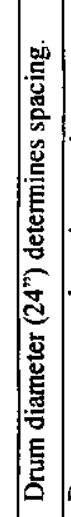 & 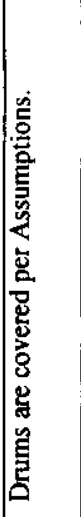 & 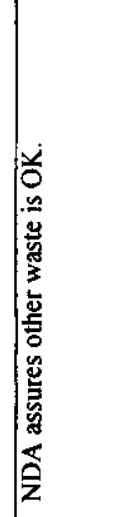 \\
\hline 总 & $\overrightarrow{0}$ & e & $\beta$ & ثر & Z & $\mid \begin{array}{l}\overrightarrow{0} \\
0\end{array}$ & s & $\overrightarrow{u^{\prime}}$ & $\vec{P}$ & $\vec{u}$ & $\overrightarrow{0}$ & $\vec{ن}$ & ثر & ن & $\vec{v}$ & in \\
\hline 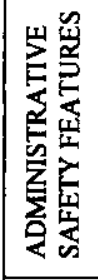 & 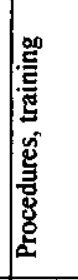 & 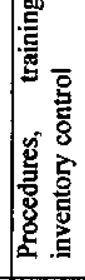 & 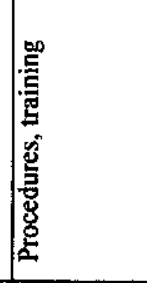 & 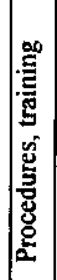 & 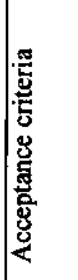 & 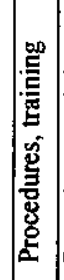 & 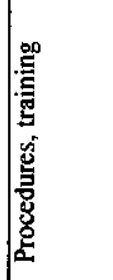 & 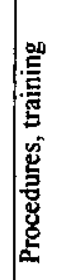 & 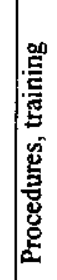 & 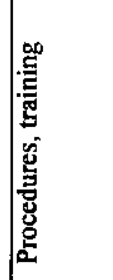 & 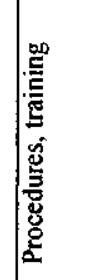 & 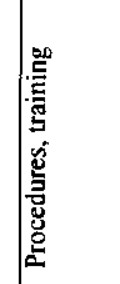 & 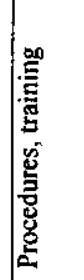 & 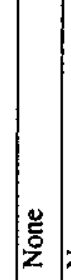 & 冚 & 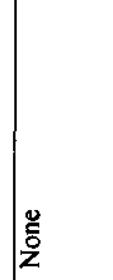 \\
\hline 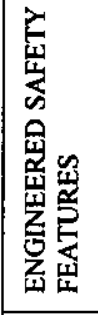 & z̆ & 产 & 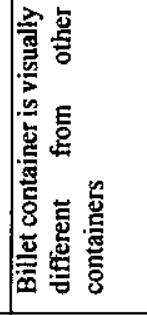 & 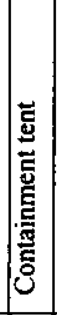 & 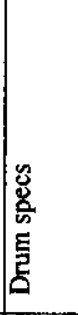 & 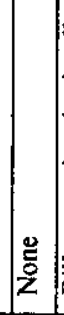 & 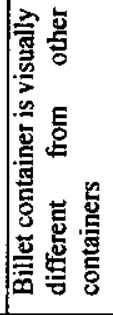 & 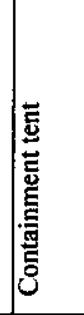 & 亗 & 䓂 & 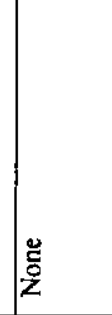 & 䓂 & 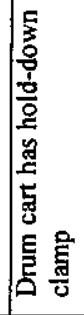 & 号 & 蒙 & z̆ \\
\hline 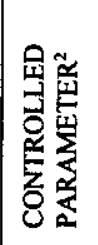 & $\mid \begin{array}{l}y \\
\Sigma\end{array}$ & 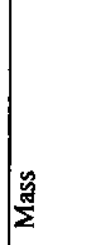 & 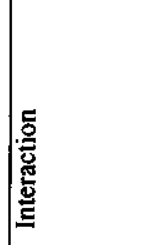 & 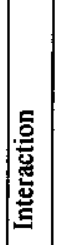 & : & 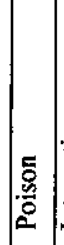 & 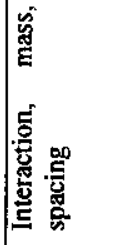 & 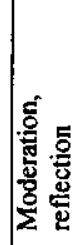 & 竧 & 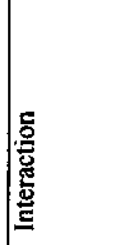 & 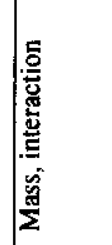 & 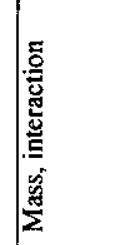 & 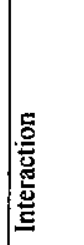 & : & 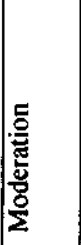 & 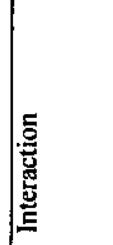 \\
\hline 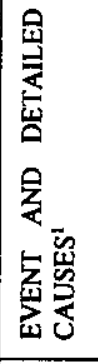 & 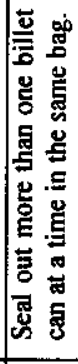 & 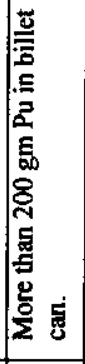 & 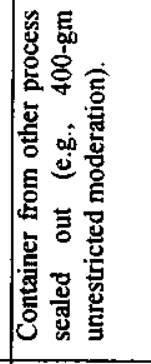 & 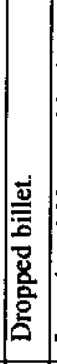 & 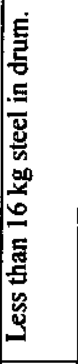 & 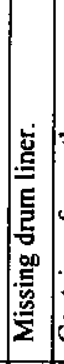 & 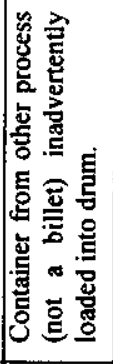 & 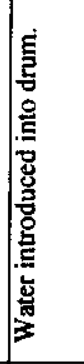 & 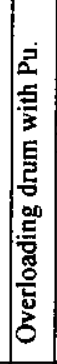 & 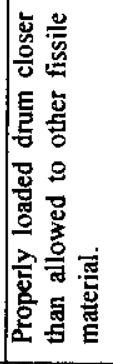 & 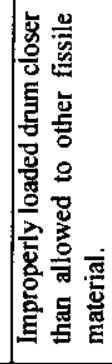 & 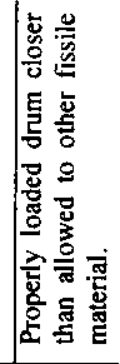 & 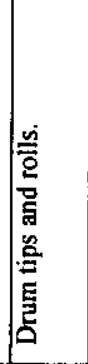 & 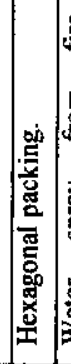 & 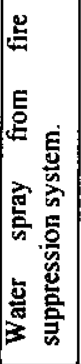 & 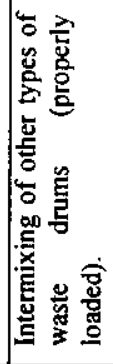 \\
\hline 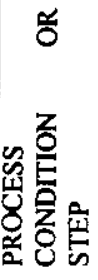 & 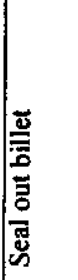 & 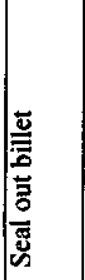 & 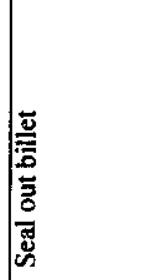 & 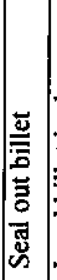 & 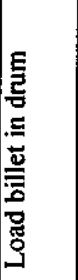 & 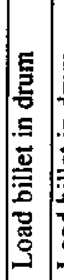 & 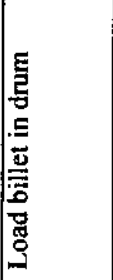 & 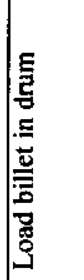 & 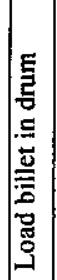 & 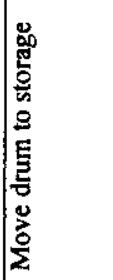 & 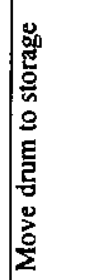 & 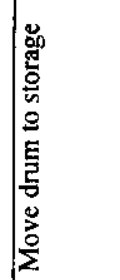 & 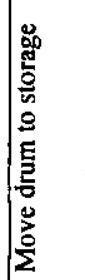 & 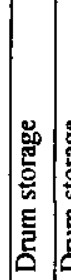 & 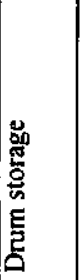 & 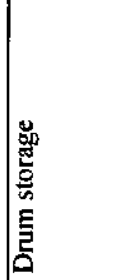 \\
\hline$\cong$ & - & $N$ & & 1 & & & & & & & $=$ & $\simeq$ & & & & 1 \\
\hline
\end{tabular}




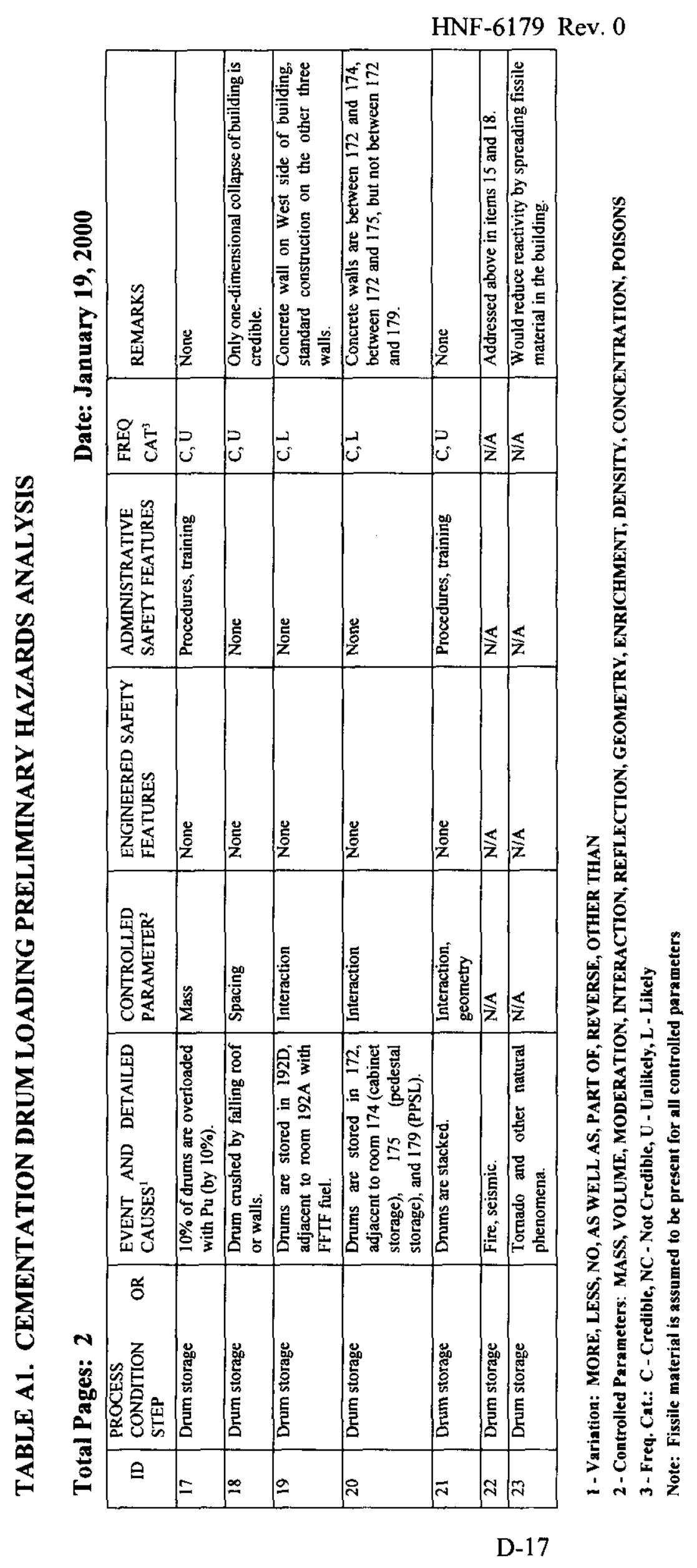


HNF-6179 Rev. 0

This page intentionally left blank.

D-18 
HNF-6179 Rev. 0

APPENDIX E - MCNP MODEL FIGURES

E-1 
HNF-6179 Rev. 0

This page intentionally left blank.

E-2 
HNF-6179 Rev. 0

Figure E-1. Wagon Next to Single Drum - Plan.

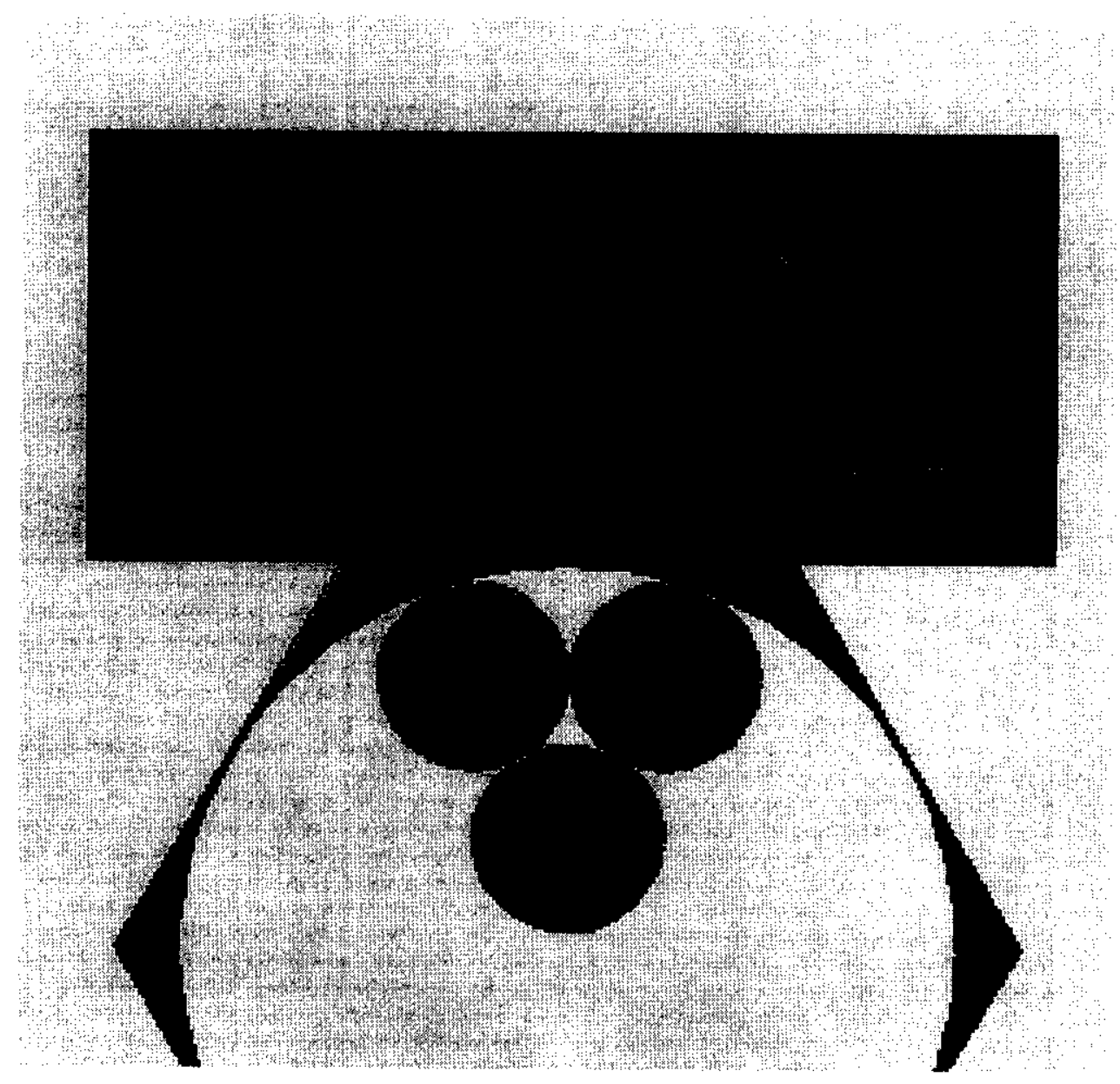




\section{HNF-6179 Rev. 0}

Figure E-2. Wagon Next to Single Drum - Elevation

(Note that only one billet is in the plane of this cut)

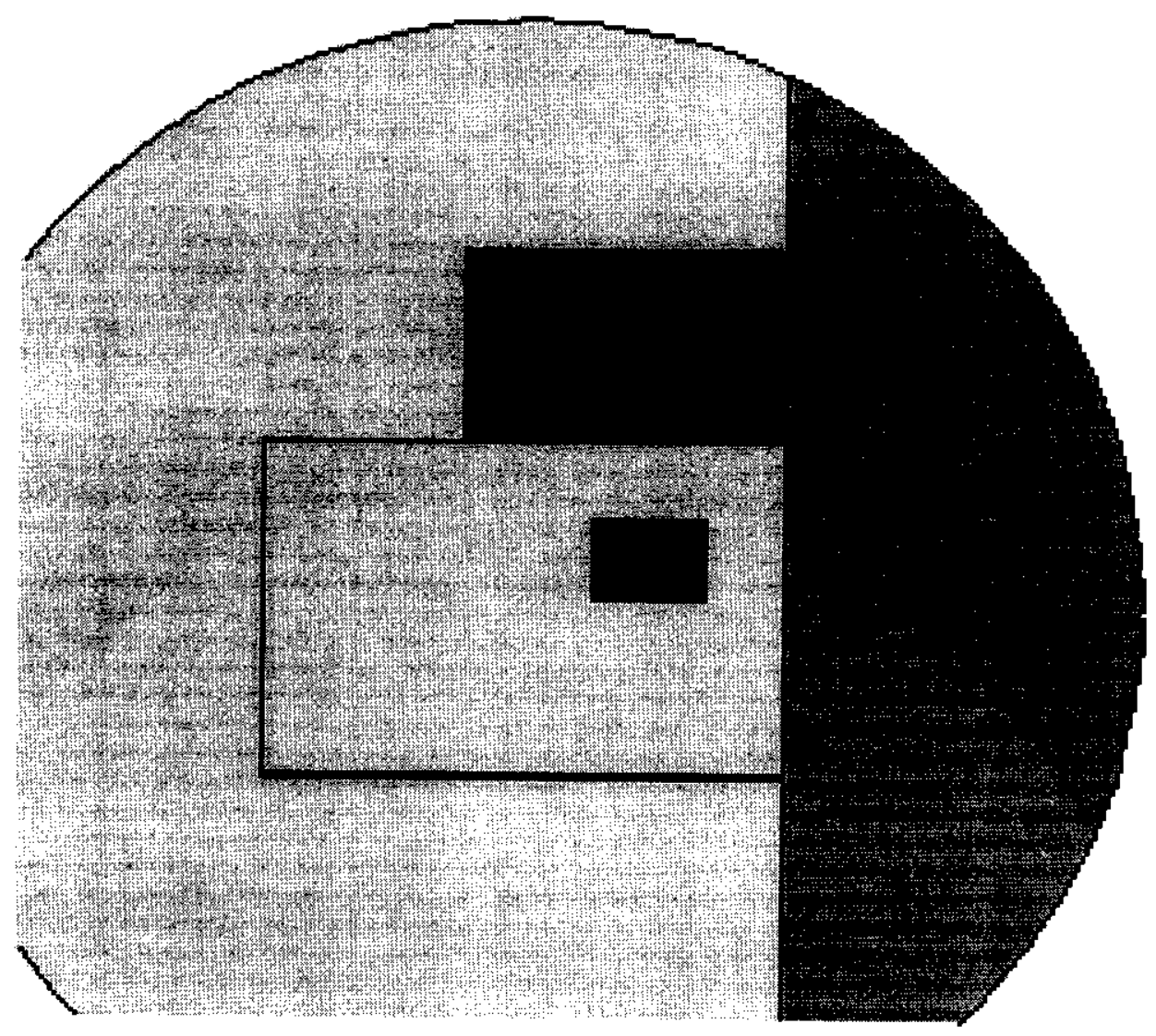




\section{HNF-6179 Rev. 0}

Figure E-3. Wagon Next to Room Array of Drums - Plan

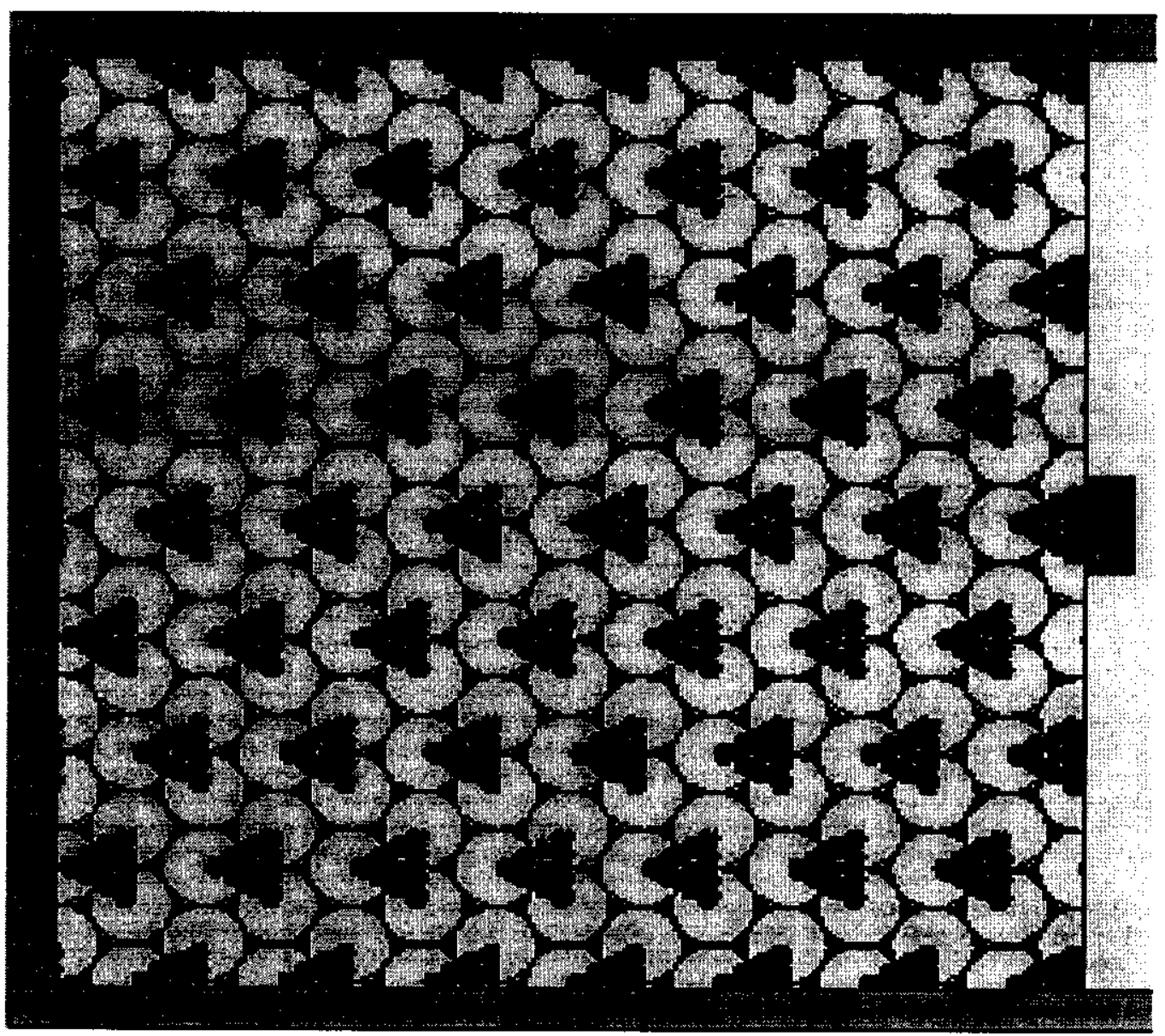


HNF-6179 Rev. 0

This page intentionally left blank. 